\title{
Finitely additive strategies, zero-sum games, and decision making
}

Citation for published version (APA):

Zseleva, A. (2018). Finitely additive strategies, zero-sum games, and decision making. [Doctoral Thesis, Maastricht University]. Datawyse / Universitaire Pers Maastricht. https://doi.org/10.26481/dis.20180208az

Document status and date:

Published: 01/01/2018

DOI:

10.26481/dis.20180208az

Document Version:

Publisher's PDF, also known as Version of record

\section{Please check the document version of this publication:}

- A submitted manuscript is the version of the article upon submission and before peer-review. There can be important differences between the submitted version and the official published version of record.

People interested in the research are advised to contact the author for the final version of the publication, or visit the DOI to the publisher's website.

- The final author version and the galley proof are versions of the publication after peer review.

- The final published version features the final layout of the paper including the volume, issue and page numbers.

Link to publication

\footnotetext{
General rights rights.

- You may freely distribute the URL identifying the publication in the public portal. please follow below link for the End User Agreement:

www.umlib.nl/taverne-license

Take down policy

If you believe that this document breaches copyright please contact us at:

repository@maastrichtuniversity.nl

providing details and we will investigate your claim.
}

Copyright and moral rights for the publications made accessible in the public portal are retained by the authors and/or other copyright owners and it is a condition of accessing publications that users recognise and abide by the legal requirements associated with these

- Users may download and print one copy of any publication from the public portal for the purpose of private study or research.

- You may not further distribute the material or use it for any profit-making activity or commercial gain

If the publication is distributed under the terms of Article $25 \mathrm{fa}$ of the Dutch Copyright Act, indicated by the "Taverne" license above, 
Finitely ADditive STRATEgIES, ZeRO-SUM GAMES, AND DECISION MAKING

ANNA ZSELEVA 
(C) Anna Zseleva, Maastricht 2017.

All rights reserved. No part of this publication may be reproduced, stored in a retrieval system, or transmitted in any form, or by any means, electronic, mechanical, photocopying, recording or otherwise, without the prior permission in writing from the author.

This book was typeset by the author using $\mathrm{ET}_{\mathrm{E}} \mathrm{X}$ and the classicthesis package.

Published by Universitaire Pers Maastricht ISBN: 9789461597977

Printed in The Netherlands by Datawyse Maastricht

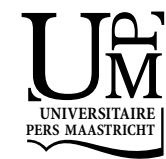




\title{
FINITELY ADDITIVE STRATEGIES, ZERO-SUM GAMES, AND DECISION MAKING
}

\author{
DISSERTATION \\ to obtain the degree of Doctor at \\ Maastricht University, \\ on the authority of the Rector Magnificus, \\ Prof. dr. Rianne M. Letschert, \\ in accordance with the decision of the Board of Deans, \\ to be defended in public \\ on Thursday 8 of February 2018, at 12.00 hours
}

by

Anna Zseleva 


\section{Supervisor}

Prof. dr. Dries Vermeulen

Co-supervisor

Dr. János Flesch

\section{Assessment Committee}

Prof. dr. Frank Thuijsman (Chair)

Dr. Miklós Pintér (University of Pécs, Hungary)

Dr. Arkadi Predtetchinski

Prof. dr. William Sudderth (University of Minnesota, USA)

This research was financially supported by the Graduate School of Business and Economics (GSBE). 
To my family for their eternal support

На семейството ми. С благодарност за вечната им подкрепа. 



\section{ACKNOWLEDGEMENTS}

I think that a good economist should be upfront about their assumptions, that is why I start this section with two of them.

- If you think that you should be mentioned here and I did not mention you, then you are right.

- If you think that I wrote too nice or cheesy things about you that are untrue, then you are wrong.

I need these assumptions because it seems impossible to mention all the wonderful people that I met along the way who helped and shaped me, possibly without even knowing it. For example, I had colleagues who always smiled or had a nice word when I passed them in the corridor which I really appreciated. Moreover, I am well aware that some of the people that I would like to thank are too kind for their own good.

First of all, I am indebted to my supervisors Dries and János. I remember the confusing first days in Maastricht when I did not really know who they were, or how to pronounce Dries' last name, but all my fellow PhDs were congratulating me on how lucky I was to get them as supervisors. Back then I could just nod along without having any idea what these PhD students were talking about. Now, four years later, I can heartily agree with these comments, and pass them on to the new PhDs (in order to confuse them as well). Unfortunately, some of my main features are constantly talking and continuously doubting everything, which means that bearing with me as a $\mathrm{PhD}$ student requires a substantial amount of patience. So I cannot only thank my supervisors for their knowledge and wisdom, but they deserve respect for their patience as well. It would be amiss if I did not mention that besides for their help in research, I am grateful for the Robo rally nights, Terry Pratchett discussions, concerts, movie nights, countless lunches and dinners. In conclusion, one usually hopes for smart and helpful supervisors, but I was lucky, because mine are also patient, caring and funny. 
I would like to thank the assessment committee for taking the time to carefully read the thesis. I am grateful to Miklós for many things, including being my master thesis supervisor, encouraging me to apply for a $\mathrm{PhD}$ position in Maastricht, and for arranging the research seminar for his students. It was a great community with in-depth discussions about research. We did not only think of Miklós as a supervisor, but also as a mentor, so special thanks for that. I would like to thank Arkadi for our inspiring discussions on measure theory, game theory, history, books and ice cream. Most importantly, I would like to thank him for sharing his never-ending supply of good quality chocolate. I was privileged that Arkadi shared his unlimited wisdom with me by sometimes saying truly profound statements such as "if I didn't have a brain, I wouldn't eat ice cream". Just to have closure on an extended argument: Arkadi, infinity does exist. I would like to thank Frank for being the chair of the committee, showing me the connection between mathematics and Donald Duck, and his respectable enthusiasm for taking pictures in Tel Aviv. I would like to thank Bill for his historical anecdotes on the finitely additive theory, and thank him and Jerri for crossing the ocean to come to my defense.

My dear paranymphs, Andrej and Hande, started the PhD journey with me and were part of my integration in Maastricht in many ways. Andrej helped me make my flat into a home, took me on bike trips and always let me scatter my thoughts across his office, while Hande joined me to Muziekgieterij for concerts, to improv and started the Balkans dinner tradition. We also had trips together to Amsterdam, Köln, Liege, Aachen, Berlin, Istanbul, New York, Washington DC and so on. I am glad that I could also be part of such historical moments as their weddings, in Minsk and in Crete, respectively. I would also like to thank Hanna and Levent for making them happy. I thank Emilia and Sophia for being adorable.

A warm word of thanks to Marc, Abhi, Angie and Swarnendu for asking me to be their paranymphs. I am grateful to Jan, Swarnendu, Nadine and Shash for being my fellow paranymphs and partners in crime in planning the presents and parties.

I am glad that I could be part of the KE department, and the legendary atmosphere on the $4^{\text {th }}$ floor. I think that the department community is great, and especially the $\mathrm{PhD}$ community with whom I had a lot of nice events together, 
like trips to Efteling, All in Echt, jazz and karaoke nights, PhD defense parties, Veerle's and Dries' weddings, Sinterklaas run, Marc's farewell biercantus, carnival, Abhi's, Andrey's and Tim's birthdays or simply dinners and movie nights. My PhD experience would not have been the same without Marc. He could be picked as a role model as a researcher, however I consider him as one of my role models due to his chill attitude. This also made him a great travel buddy for our trips to Singapore, Vietnam, Malaysia, Brazil, Argentina, Paris, Istanbul, Sicily, Chile and Pinkpop. It is difficult to mention all the great fellow PhDs that made my Maastricht experience. When I started there were older PhDs that made an effort to share their experiences, for example Artem and Greg. There were also the PhDs with whom we started together and stumbled through the beginning: Abhinaba, Andrej, Chris, Hande, Hanno, Kutay and Tim. Sincere thanks to Angie and Chris for the game theory dinners and for being my relentless cheerleaders, it is an amazing feeling. Thank you, Tim, for being so kind and upbeat. It was fun to discuss with Hanno econometrics, game theory, modern physics and how stupid it is to make life plans. Who would have thought that moving to the Netherlands would make me read books by the Hungarian author Márai Sándor? Thanks, Hanno. Thank you, Veerle, for the conflicting pieces of advice, thus making me use a Fitbit, but also teaching me how to make oreo cupcakes. I would like to thank Sean for his infectious laughs, Yuliya for the unicycling and slacklining, and Shash for having the confidence to proclaim himself the "coolest Indian". I thank Caterina for being a great office mate, hosting me in Rome and most importantly, for Bongo Mondays. I thank Benoit for being positive and funny and Dasha for being enthusiastic. Moreover, Adi, Aida, Ahmad, Alex, Andrey, Etiënne, Indra, Kouma, Marina, Martijn, Murat, Niels, Oksana, Rasmus, Roli, Verena, Vincent and Yicong were also great colleagues. We often took over Karin and Yolanda's office with tea breaks and superfluous questions, so thank you for the patience in dealing with us.

Teaching is an interesting part of a $\mathrm{PhD}$. The purpose is rather unclear... Is it for PhDs to socialize more? To feel more time pressure? To avoid lunches with awkward silence and fill them with amusing stories? Four years were not enough to figure this out, even though I tried by discussing this with my amazing fellow teachers: Alex, Christian, Dirk, Dries, Greg, Hanno, Hans dG, 
Hans P, János, Marc, Stan, Swarnendu, Veerle and Yuliya. Unfortunately, a few times I had to miss out on teaching due to conferences or Kati's wedding, so I thank Caterina, Chris, Dries, Hans dG and Sean for helping me out in those cases.

I think that the seminars and reading groups also contributed to building a community. I would like to thank my MLSE coorganizers, Mehmet, Zsombor, Hande, Marc and Anastas, and the members of the epistemic game theory and modal logic reading groups, Abhi, Andrés, Angie, Chris, Elias, Marc, Swarnendu...

SBE surprisingly does not only consist of the KE department. People from AE1 and ROA also made my time there fun, especially Amber, Evi, Frauke, Henrik, Marcello, Maria, Marion, Nick, Peti, Riccardo and Sanne. I thank Jan and Nico for our travels in Australia and the insightful discussions on behavioral economics, the concept of 3-am-friends, improv and life. Alexandra was a great addition to these discussions.

My life in Maastricht would not have been the same without PhD improv, and I need to thank Gabri and Nevena for forcing me to go even though I had zero initial inclination. I cherish the memories of the Thursday evening improv sessions, birthday and costume parties, trips to Belgium, Groningen, Paris and Tenerife. It would be hard to over emphasize the effect of the improv community on me. It is impossible to mention every single person who attended a session with me and made me laugh. I would like to thank Jessie, Mare and Norbert for being so incredibly caring, Mehrdad and Sanne for the surreal conversations, and Frederik for the trip to Tel Aviv. Just to mention at least some of the incredibly amusing people that were part of the improv journey: Adam, Bas, Burcu, Dorijn, Eloy, Elsa, Eveline, Fred, Gintare, Hande, Jan, Julien, Jo-Anne, Kutay, Latifa, Mahdi, Martina, Masha, Mueid, Nordin, Olga, Paola, Shuan, Sofya, Tobi, Victor and Zubin. PhD Acamedy in general also organized many movie nights, dance workshops and sports events. I thank all the committee members who organized these events, and the people with whom we hung out there. 
I would like to thank Marco Scarsini for hosting me in Rome, Marco Dall'Aglio for sharing his ideas about lego, Antonio, Francesco, Giulia and Stefano for taking me to concerts and Elena, Natasha and Rosanna for the exotic dinners. A lot of great memories stem from conferences (see above the list of travels with Marc), so I would like to thank colleagues with whom we met at conferences and had nice discussions. For example, Bruno, Gaëton and Xavier were part of my adventures in New York and Paris. Despite these disastrous trips I would like to thank them for their sense of humour which makes them continuous sources of entertainment.

I appreciate that some of my friends and relatives bothered to come to Maastricht, even though initially it might not seem like the most exciting place on the planet: Kata, Kati, Ádám, Ели, Бояна, Наско, Dani, Oli, Denise, Mánuel, Klári, Gabi, Györgyi and Zsolti. Besides that I'm happy that we kept in touch with my fellow bachelor students, for example Adri, Feri, Gábor, Marci, Olgi, Tomi, Zsófi and high school friends Ági, Balázs, Bogi and Mezei. I'm also thankful to my friends and relatives who got married in the last few years, and gave me a chance to combine traveling with an awesome party.

I thank Mehrdad for showing me the physics related video called "Taming infinity", and Kati for designing my cover based on it.

Surprisingly, there is life even after Maastricht, and for me specifically in Saint Petersburg. I would like to thank my new colleagues for being so incredibly inclusive, especially Alex, Anna, Fedor and Xenia.

Finally, I would like to thank my family. Благодаря на баба Радка и на дядо Йонко, че възпитаха майка ми да е толкова грижлива. А на мама съм благодарна, че се грижи толкова много за мен. Köszönöm Nagymamának a "sütit" és a sok törődést, és Nagyapának, hogy sosem etetett velünk fokhagymatortát. Köszönöm Apunak és Bobinak, hogy megtanítottak rá, hogy semmit és senkit sem szabad komolyan venni, de azért mindig segítőkészek, ha tanácsra van szükségem. Благодаря на братовчедите си, на Ели, Никола, Бояна, Пухи и на семействата им. A Bencsik-klánnak is szeretnék mindent megköszönni. Mátét még nem ismerem olyan jól, de a szülei alapján arra számítok, hogy ő is nagyon kedves és pozitív emberke lesz. 



\section{CONTENTS}

ACKNOWLEDGEMENTS vii

1 INTRODUCTION

2 PRELIMINARIES $\quad 5$

3 ZERO-SUM GAMES WITH CHARGES $\quad 7$

3.1 Introduction . . . . . . . . . . 7

3.2 Preliminaries..................... 11

3.3 Games with charges ... . . . . . . . . . 12

3.3.1 Basic definitions ................. 13

3.3.2 Optimistic and pessimistic players .......... 14

3.3 .3 Double integrals ................. 15

3.3 .4 All games are different . . . . . . . . . . 16

3.4 Guarantee level ....................... 19

3.4.1 Definitions ................... 19

3.4 .2 Finitistic guarantee levels ............. 20

3.4 .3 The main result . . . . . . . . . . . . . 21

3.4 .4 The classical guarantee level . . . . . . . . . . 22

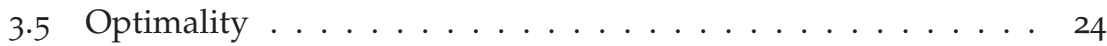

3.5 .1 Optimistic optimality . . . . . . . . . 24

3.5 .2 Pessimistic optimality . . . . . . . . . . . 28

$3 \cdot 5 \cdot 3$ Optimality for double integrals . . . . . . . . 30

3.6 Zero-sum games with charges . . . . . . . . . . . 30

3.7 Concluding remarks . . . . . . . . . . . . 32

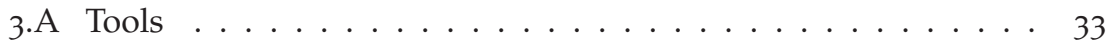

3. B Proof of Theorem $3.4 .3 \ldots \ldots \ldots \ldots$

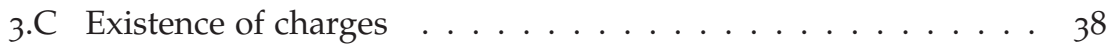

4 CATCH GAMES - THE IMPACT OF MODELING DECISIONS 43

4.1 Introduction . . . . . . . . . . . . 43

4.2 Preliminaries........................ 47

4.3 Catch games . . . . . . . . . . . . . . . 48

4.4 The main results . . . . . . . . . . . . . 51

4.5 Consequences from set theory . . . . . . . . . . . 53 


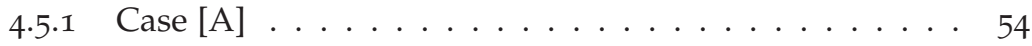

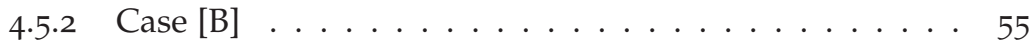

4.5 .3 Case $[C] \ldots \ldots \ldots \ldots \ldots$

4.5 .4 An example . . . . . . . . . . . . . . . 56

4.6 Finitely additive strategies . . . . . . . . . . . . . . . 57

4.7 Remarks on our requirements for the sigma-algebras . . . . . 58

4.8 Concluding remarks . . . . . . . . . . . . . . . . . 59

4.A The proofs of Theorems 4.4.1 and $4.4 .2 \ldots \ldots \ldots \ldots$. . . . 61

4. B Toolbox .................... 70

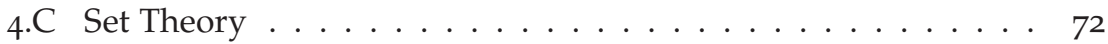

5 Finitely ADDitive MiXed AND BeHAVIOR STRATEgIES IN INFINITE DURATION DECISION PROBLEMS 77

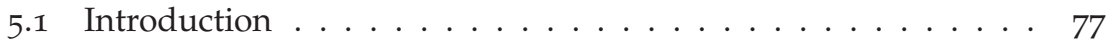

5.2 The model . . . . . . . . . . . . . . . . . . 80

5.3 Examples ..................... 81

5.4 Topological issues . . . . . . . . . . . . . . . . . . . . 86

5.5 Induced charges on the set P of plays and expected payoffs . . 89

5.5.1 Mixed strategies . . . . . . . . . . . . . . 9 90

5.5 .2 Behavior strategies . . . . . . . . . . . . . 91

5.6 Four approaches to induce charges on the set $\mathrm{P}$ of plays by behavior strategies . . . . . . . . . . . . . . . . 93

5.6.1 Approach I: the minimal competent algebra $\mathcal{R}^{\text {hs }} \ldots .93$

5.6.2 Approach II: stop rules . . . . . . . . . . . . . . . . . . 94

5.6.3 Approach III: stop rules and singletons . . . . . . . . . 96

5.6 .4 Approach IV: open sets _. . . . . . . . . . . . . . 98

5.7 The main results . . . . . . . . . . . . . . . . 101

5.8 Concluding remarks . . . . . . . . . . . . . . . . . 109

5. A The proofs .................. 110

5. B Auxiliary claims . . . . . . . . . . . . . . . 130

6 CONCLUSIONS 133

BIBLIOGRAPHY $\quad 135$

INDEX 143

SUMMARY 145

VALORISATION 149

CURRICULUM VITAE 


\section{INTRODUCTION}

A noncooperative game is a model of strategic interaction between players. A player's payoff might depend on the decisions taken by other players. Decision theory can be viewed as a special case of game theory where there is only one player, called a decision maker. Game theory has many applications in economics, biology, computer science, logic, political science and psychology. ZERO-SUM GAMES In zero-sum games there are two players. Given any pair of strategies of the players, the payoffs of the two players add up to zero. Hence, it is sufficient to consider the payoff of player 1 and also interpret it as the payment of player 2. These types of games are also called strictly competitive or conflict games.

The value is the central solution concept of zero-sum games. If a zero-sum game has a value, then this is the highest amount that player 1 can guarantee to receive, irrespective of what the other player plays. Similarly, the value is the lowest amount that player 2 can guarantee to pay, irrespective of what player 1 plays.

The theory of zero-sum games starts with von Neumann (1928), who showed that zero-sum games with finite action spaces admit a value. Games with infinite action spaces are much more complex, and Wald (1945) demonstrated that such games do not always have a value. In Wald's game there are two players who can each say any natural number. Player 1 wins, if he says a higher number or the same as player 2, otherwise he loses.

probabilities When a player makes a choice, he bases his decision on probabilities. In game theory the usual approach is to define mixed strategies as countably additive probabilities on the actions. A notable, but less frequent alternative is to define mixed strategies as finitely additive probabilities, socalled charges. Since finite additivity is a weaker requirement than countable additivity, the latter approach allows for a richer class of mixed strategies. 
Charges have regularly been argued for from a conceptual point of view, but they also provide technical advantages.

STRUCTURE OF THE THESIS Chapter 2 is a preliminary chapter, it mainly focuses on notions connected to finitely additive probabilities.

Chapters 3 and 4 are about zero-sum games with infinite action spaces. In Chapter 3 we consider any game with a bounded payoff function, and mainly focus on finitely additive strategies. In Chapter 4 we consider only a certain type of zero-sum games, and focus on countably additive strategies.

In Chapter 3 we consider two-player zero-sum games with infinite action spaces and bounded payoff functions. The players' strategies are finitely additive probability measures, called charges. Since a strategy profile does not always induce a unique expected payoff, we distinguish two extreme attitudes of players. A player is viewed as pessimistic if he always evaluates the range of possible expected payoffs by the worst one, and a player is viewed as optimistic if he always evaluates it by the best one. This approach results in a definition of a pessimistic and an optimistic guarantee level for each player. We provide an extensive analysis of the relation between these guarantee levels, and connect them to the guarantee levels defined through countably additive strategies, and to other known techniques to define expected payoffs, based on computation of double integrals. In addition, we also examine existence of optimal strategies with respect to these guarantee levels.

Chapter 3 is centered around the problem that a finitely additive strategy profile does not always induce a unique expected payoff. One might reach the conclusion that finitely additive strategies are problematic, and so their use should be avoided. In Chapter 3 we only compute the guarantee levels through countably additive strategies when the action space is countable. When a game has action spaces with larger cardinalities, countably additive strategies can cause similar problems to finitely additive strategies. This is investigated in Chapter 4.

In Chapter 4 we examine the guarantee levels defined through countably additive strategies of the players in a type of zero-sum games, catch games. We show how these levels depend on the sigma-algebras that are being employed on the player's action spaces. We further argue that guarantee levels 
may therefore also depend on set theoretic considerations. Additionally, we calculate the guarantee levels for finitely additive strategies. The solutions of catch games essentially differ among these setups. We find optimal strategies for almost all cases.

In Chapters 3 and 4 we consider two-player games, however Chapter 5 includes a game with one player, so a decision theoretic model. Chapters 3 and 4 consider games where players move once and simultaneously. In Chapter 5 the decision maker is taking decisions over infinitely many time periods.

In Chapter 5 we consider the following class of decision problems. A decision maker chooses an action $a_{t}$ from a given action space $A$ at every period $t=1,2, \ldots$, and receives a payoff that is a function of the resulting sequence $\left(a_{1}, a_{2}, \ldots\right)$. A mixed strategy of the decision maker is a finitely additive probability measure on the space of pure strategies. A behavior strategy of the decision maker is a mapping that assigns to every history a finitely additive probability measure on the action space. In this setup, we address several questions, both from a conceptual and from a technical point of view. For a behavior strategy, it is generally not clear which finitely additive measure such a strategy induces on the set of plays, i.e., on the set of all infinite sequences of elements of $A$. Consequently, it is not clear which mixed strategies are equivalent to the given behavior strategy, and therefore what the expected payoff should be. We present and compare various approaches to this problem. Moreover, we investigate the equivalence between behavior and mixed strategies.

related literature There are different ways of defining probabilities. Countable additivity is a usual assumption of probability. However, from a conceptual point of view, the weaker assumption of finite additivity was also argued for, see de Finetti (1975), Savage (1972), and Dubins and Savage (2014). Since finite additivity is the weaker assumption, it is also more basic. It allows for a richer class of probabilities, and facilitates constructions such as a uniform probability distribution over the natural numbers (see Schirokauer and Kadane (2007)). Moreover, translation invariant charges can be defined on all subsets of the real line, and translation and rotation invariant charges can be defined on all subsets of the plane. A notable advantage of finitely additive measures, more briefly called charges, is that they avoid the problem 
of measure (cf. Aliprantis and Border (2005)). Finally, in decision theory and game theory, only approximate solutions may be achieved with countably additive strategies, while exact solutions can be achieved when using charges (e.g. Sudderth (2016)). For a more extensive summary of the history of finite additivity and its relation to countable additivity, see Bingham (2010). 


\section{PRELIMINARIES}

In this section we provide a brief summary on probability charges. For further reading, we refer to Rao and Rao (1983) and Dunford and Schwartz (1964).

Let $\mathrm{X}$ be a nonempty set. A collection $\mathscr{P}$ of subsets of $\mathrm{X}$ is called an algebra if it has the following propertis: (1) $\mathrm{X} \in \mathscr{P}$, (2) if $\mathrm{E}, \mathrm{F} \in \mathscr{P}$, then $\mathrm{E} \cup \mathrm{F} \in \mathscr{P}$, (3) if $\mathrm{E} \in \mathscr{P}$, then $\mathrm{E}^{\mathrm{c}} \in \mathscr{P}$. It follows that an algebra is also closed under taking finite unions. An algebra is called a sigma-algebra, if it is even closed under taking countable unions.

For example, the set $2^{X}$, i.e. the set of all subsets of $X$, is a sigma-algebra. However, there are algebras that are not sigma-algebras. For example, take an infinite set $X$ and the algebra consisting of all finite subsets of $X$ and their complements.

Let $\mathscr{P}$ be an algebra on $\mathrm{X}$. A probability charge, or simply charge, on $(\mathrm{X}, \mathscr{P})$ is a function $\mu: \mathscr{P} \rightarrow[0,1]$ such that $\mu(X)=1$ and for all disjoint sets $E, F \in \mathscr{P}$ it holds that $\mu(E \cup F)=\mu(E)+\mu(F) .{ }^{1}$ We denote the set of all charges on $(\mathrm{X}, \mathscr{P})$ by $\mathcal{C}(\mathrm{X}, \mathscr{P})$.

The following statement follows from Theorem 2 in Loś and Marczewski (1949) together with the Lemma of Zorn, and is also shown in Theorem 3.C.3. If $\mathscr{P}$ is an algebra, and $\mu$ is a charge on $\mathscr{P}$, then $\mu$ can be extended to a charge on $2^{X}$. That is, there exists a charge $v$ on $\left(X, 2^{X}\right)$ such that $\nu(E)=\mu(E)$ for all $\mathrm{E} \in \mathscr{P}$.

Let $\mathscr{P}$ be a sigma-algebra on X. A (countably additive) probability measure on $(X, \mathscr{P})$ is a function $\mu: \mathscr{P} \rightarrow[0,1]$ such that $\mu(X)=1$ and if for all collections $\left\{E_{i}\right\}_{i=1}^{\infty}$ of pairwise disjoint sets in $\mathscr{P}$ it holds that $\mu\left(\cup_{i=1}^{\infty} E_{i}\right)=\sum_{i=1}^{\infty} \mu\left(E_{i}\right)$. We denote the set of all probability measures on $(X, \mathscr{P})$ by $\Delta(X, \mathscr{P})$.

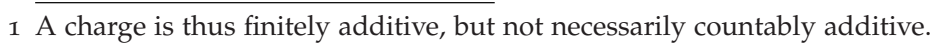


For $x \in X, \delta_{x}$ denotes the Dirac measure on $x$. So, for every set $B \in \mathscr{P}$, we have $\delta_{x}(B)=1$ if $x \in B$ and $\delta_{x}(B)=0$ if $x \notin B$. Note that $\delta_{x} \in \Delta(X, \mathscr{P})$ for every $x \in X$.

Let $\mathscr{P}$ be an algebra on $\mathrm{X}$. A function $s: \mathrm{X} \rightarrow \mathbb{R}$ is called a $\mathscr{P}$-measurable simple-function if there are $\mathrm{c}_{1}, \ldots, \mathrm{c}_{\mathrm{m}} \in \mathbb{R}$ and a partition $\left\{\mathrm{B}_{1}, \ldots, \mathrm{B}_{\mathrm{m}}\right\}$ of $\mathrm{X}$ with $B_{1}, \ldots, B_{m} \in \mathscr{P}$ such that $s=\sum_{i=1}^{m} c_{i} \mathbb{I}_{B_{i}}$, where $\mathbb{I}_{B_{i}}$ is the characteristic function of the set $B_{i}$. Let $\mu$ be a charge on $(X, \mathscr{P})$. The integral of $s$ with respect to the charge $\mu$ is defined by $\int_{X} s d \mu=\sum_{i=1}^{m} c_{i} \cdot \mu\left(B_{i}\right)$.

Let $\mu$ be a charge on $\left(X, 2^{X}\right)$. For every bounded function $f: X \rightarrow \mathbb{R}$ and every $\varepsilon>0$, there exists a $\left(2^{X}\right.$-measurable) simple-function s such that $s \leqslant f \leqslant s+\varepsilon$. ${ }^{2}$ Let $f: X \rightarrow \mathbb{R}$ be a bounded function. The integral $\int_{X} f d \mu$ is defined as the supremum of all real numbers $\int_{X} s d \mu$, where $s$ is a simple-function with $s \leqslant f$. Since $f$ is bounded, the integral is finite. The integral is linear over the set of bounded real-valued functions. We remark that the integral $\int_{X} f d \mu$ is equal to the infimum of all real numbers $\int_{X} s \mathrm{~d} \mu$, where $s$ is a simple-function and $s \geqslant f$.

When $X$ is countably infinite, we say that $\mu \in \mathcal{E}\left(X, 2^{X}\right)$ is a pure charge if $\mu(\{x\})=0$ for every $x \in X .{ }^{3}$ It follows from Theorem 3.C.3 that pure charges exist. ${ }^{4}$ Since pure charges are not countably additive, it follows that $\Delta\left(X, 2^{X}\right)$ is a strict subset of $\mathcal{C}\left(X, 2^{X}\right)$ in this case.

2 See p. 272 in the appendix by WD Sudderth, D Gilat and R Purves in Dubins and Savage (2014). Indeed, consider the inverse images $f^{-1}[z \varepsilon,(z+1) \varepsilon)$, where $z$ is an integer. Since $f$ is bounded, only finitely many of them are non-empty. If $f^{-1}[z \varepsilon,(z+1) \varepsilon)$ is non-empty, then let $s$ take value $z \mathcal{E}$ on this set. It follows that $s \leqslant f \leqslant s+\varepsilon$.

3 The definition of a pure charge can be generalized to sets that are uncountably infinite. However, in the general case the definition is more involved.

4 In Theorem 3.C. 3 we can take for instance $\mathcal{F}$ to be the algebra of all finite subsets of $X$ and their complements. 


\subsection{INTRODUCTION}

We have shortly mentioned the origins of zero-sum games in Chapter 1 . When the action spaces are infinite, the definition of mixed strategies is not trivial. In game theory the usual approach is to define mixed strategies as countably additive probabilities on the actions. In this chapter however we focus our attention on finitely additive probabilities, so-called charges.

In this chapter ${ }^{1}$, we are interested in the best expected payoff that a charge can guarantee for a player. Since the expected payoff can be defined in various manners, we obtain several guarantee levels. Our goal is to examine these guarantee levels in detail, and to find conditions under which the value of a game exists.

OUR SETUP We study general two-player zero-sum games with infinite, possibly uncountable, action spaces and bounded payoff functions. The strategy space of a player is the set of probability charges defined on all subsets of that player's action space. The game is now played as follows. Each player simultaneously selects a probability charge on his action space. These choices are then recorded in a strategy profile. An extension of such a profile is a probability charge that is defined on all subsets of the set of action profiles that coincides on product sets (rectangles) with the product of the strategies.

Given a strategy profile of probability charges, there may be many such extensions. And any extension induces an expected payoff to each player, so that the expected payoff may not be uniquely defined. Since the set of expected payoffs we can thus construct is not necessarily singleton, we study several

1 This chapter is based on Flesch, Vermeulen and Zseleva (2017). We would like to thank Valerio Capraro, Ferenc Forgó, Ehud Lehrer, Christian Nauerz, Miklós Pintér, Marco Scarsini, and William Sudderth for their helpful comments and discussion prior to that publication. We also thank the Dutch institute NWO for funding the research visit of William Sudderth. 
ways - some of them novel, some of them introduced before-to define the value of the resulting game, or better, class of possible games.

The first two-novel—notions relate to two extreme attitudes of players. In our framework, a player is viewed as pessimistic if from the range of possible expected payoffs he always selects the worst one. Equivalently, he always selects an extension that results in the worst possible payoff. ${ }^{2}$ In contrast, a player is viewed as optimistic if from the range of possible expected payoffs he always selects the best one. This approach results in a definition of a pessimistic and an optimistic guarantee level for each player.

The second pair of notions relate to the two possible ways to compute double integrals with respect to the chosen probability charges. Each double integral assigns an expected payoff to both players. Note that for these notions the choice of extension depends only on the strategy profile, not on the payoff function of the zero-sum game.

our CONTRIBUtion Starting with a given zero-sum game, endowing each player with one of the above approaches to compute expected payoffs yields a new game. We argue that, given a zero-sum game, the resulting games may all be different. In each such a resulting game, a player can compute the maximum payoff he can guarantee for himself, which leads to a guarantee level for that player. We provide an extensive analysis of the relation between these guarantee levels. In addition, we also examine existence of optimal strategies with respect to these guarantee levels. Our main findings are:

[1] The pessimistic guarantee level for player 1 , the optimistic guarantee level for player 2, and the guarantee levels for both players in which we first integrate with respect to the strategy of the $2^{\text {nd }}$ player and then with respect to the $1^{\text {st }}$ player, all coincide. Similarly, the optimistic guarantee level for player 1 , the pessimistic guarantee level for player 2 and the guarantee levels arising from the reverse order of integration all coincide as well (cf. Theorem 3.4.3).

2 Note that this type of selection of the extension crucially depends on the payoffs of the game, not merely on the strategy profile that is chosen. 
[2] Moreover, the guarantee levels defined above can be calculated by computing so-called finitistic guarantee levels, associated with finite approximations of the resulting games (cf. Theorem 3.4.3).

[3] Thus, when one player is optimistic, and the other is pessimistic, for any zero-sum game we start with, the resulting (zero-sum) game has a value. The same holds when both players use the same order of integration for the approach based on double integrals: the resulting game is still zero-sum, and the value exists (cf. Corollary 3.6.1 and 3.6.2).

This is a novel result in the sense that we establish an existence result for a class of payoff functions that is outside the scope of earlier papers such as Capraro and Scarsini (2013), Harris et al (2005), Marinacci (1997) and Reny (1999).

A counterexample illustrates that it is not a general fact that the game has a value when the resulting game is zero-sum. We construct a zero-sum game (a game in which the players agree on how to compute expected payoffs) in which the guarantee levels for the players do not coincide. In other words, the resulting zero-sum game does not have a value (cf. Example 3.6.3).

[4] We prove that players always have optimal strategies with respect to the optimistic guarantee level (cf. Theorem 3.5.2). We also show that a pessimistic optimal strategy of a player, when it exists, ${ }^{3}$ is also optimal for that player when he computes payoffs by a double integral where the integration is first over strategies of his opponent (cf. Proposition 3.5.8).

[5] For countable action spaces we show that the classical guarantee level for a player, when players only play countably additive probability distributions, coincides with the pessimistic guarantee level of that player (cf. Theorem 3.4.5).

Structure of the chapter In Section 3.2 we discuss some preliminaries. In Section 3.3 we introduce the zero-sum games we consider and the different ways of calculating expected payoffs. Section 3.4 contains our solution concepts and main results. Section 3.5 deals with optimal strategies, and Section 3.6 is about games where the players consider the same extension.

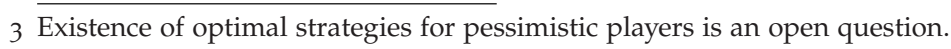


Related Literature There is a Related literature section in Chapter 1 on probabilities.

Decision theory and game theory In decision theory, charges have been used in various models, notably in de Finetti (1975), Savage (1972) and Dubins and Savage (2014). Charges also gained recognition in game theory, such as in Marinacci (1997), Capraro and Scarsini (2013), Harris et al (2005), and Maitra and Sudderth (1993), Maitra and Sudderth (1998), but to a lesser extent. One possible reason is that in a game setting it can occur that multiple players choose actions simultaneously. If each of them uses a probability charge for the choice of his action, then on the space of action profiles, as pointed out above, those choices induce a unique charge only on the algebra generated by the cylinder sets. This algebra is fairly restricted, and consequently, there is only a limited class of payoff functions for which the expected payoff is uniquely defined. For instance, Marinacci (1997) and Harris et al (2005) consider only payoff functions that can be uniformly approximated by simple-functions on this algebra.

In our setup we study a wider range of utility functions, however we consider only games that are zero-sum. As mentioned before, Marinacci (1997) and Harris et al (2005) consider a restricted class of utility functions where the extension is unique. In this setting they prove the existence of Nash-equilibrium in nonzero-sum games. Capraro and Scarsini (2013) take specific types of extensions, specific classes of payoff functions and also prove existence of Nashequilibrium in nonzero-sum games. These results indicate that generalization of our results to nonzero-sum games may require a considerable restriction of the set of utility functions.

Relating to our first pair of notions, a well-known definition of pessimism by Wald (1950) is when the decision maker assumes that whichever act he might choose, the worst state will be realised. In our framework, the pessimistic player assumes that from a range of possible expected payoffs the worst one will be realised.

Relating to our second pair of notions, taking a fixed order of integration as the expected payoff has been analyzed before by Heath and Sudderth (1972), and Maitra and Sudderth (1993), Maitra and Sudderth (1998). Yanovskaya 
(1970) and Kindler (1983) consider the double integral as an expected payoff only when different orders of integration yield the same expected payoff. For all other strategy profiles, i.e. when the order of integration does make a difference, they set an arbitrary number as an expected payoff. Schervish and Seidenfeld (1996) take convex combinations of the two orders of integration in zero-sum games, and take those as possible expected payoffs. Capraro and Scarsini (2013) also look at convex combinations of different orders of integration, but they consider countable n-player nonzero-sum games defined through an algebraic operator. We compare their approach to ours through Example 3.3.8.

As mentioned earlier, when the players agree on how to compute expected payoffs, the resulting game is zero-sum. However, this is not true in general when players disagree on the computation. This is comparable to the model of Aumann (1974), where the players might assign different subjective probabilities to the same event, and consequently, the resulting game might not be zero-sum anymore.

Ambiguity Our pessimistic approach is somewhat related to ambiguity. For example, the maximin expected utility model proposed by Gilboa and Schmeidler (1989) also computes the lowest expected utility over a set of possible probability distributions.

When using charges as strategies in game theory, one of the intriguing questions is how to define an integral over the product of action spaces. A problem of integration also arises when modeling ambiguity through non-additive probabilities, so-called capacities. Schmeidler (1989) made use of the Choquet integral, and Lehrer (2009) defined a concave integral, which can be interpreted as uncertainty aversion.

\subsection{PRELIMINARIES}

For more on probability charges, see Chapter 2.

Let $X$ be a nonempty set. By $2^{X}$ denote the set of all subsets of $X$. The set of all finite subsets of $X$ is denoted by $\mathcal{F}(X)$. In this chapter we use a shorthand, 
and we simply write $\mathcal{C}(X)$ instead of $\mathcal{C}\left(X, 2^{X}\right)$, and we use the notation $\Delta(X)$ for the set of probability measures on $\left(X, 2^{X}\right)$. Thus, $\Delta(X) \subseteq \mathcal{C}(X)$.

When $X$ is countably infinite, we say that $\mu \in \mathfrak{C}(X)$ is a pure probability charge if $\mu(\{x\})=0$ for every $x \in X$. We denote the set of all pure probability charges on $X$ by $\mathcal{Q}(X)$. Since pure charges are not countably additive, this shows that in this case $\Delta(X)$ is a strict subset of $\mathcal{C}(X)$.

We write $\mathbb{N}=\{1,2, \ldots\}$. In this chapter we assume the Axiom of Choice.

\subsection{GAMES WITH CHARGES}

In this section, we define the model of games with charges.

We focus on two-player zero-sum games. For $i=1,2$, the set $A_{i}$ is the set of actions of player $i$. The sets $A_{i}$ are not empty, and may be, but need not be, infinite. Let $u: A_{1} \times A_{2} \rightarrow \mathbb{R}$ be a bounded payoff function. The zero-sum game $\mathrm{g}(\mathfrak{u})$ is a game that is played as follows. Player 1 chooses an action $m \in A_{1}$ and independently player 2 chooses an action $n \in A_{2}$. Subsequently, player 1 receives a payoff $u(m, n)$ from player 2 . Then the game ends.

A special class of zero-sum games are binary games. Consider a set $W \subseteq$ $A_{1} \times A_{2}$. The set $W$ is called the winning set of player 1 . In the binary game defined by $W$, the payoff function $u: A_{1} \times A_{2} \rightarrow\{0,1\}$ is the characteristic function of $W$. That is, $u(m, n)=1$ when $(m, n) \in W$, and $u(m, n)=0$ otherwise.

Example 3.3.1. The following binary game is a version of the Wald game (Wald (1945)). The action sets are $A_{1}=A_{2}=\mathbb{N}$. The payoff for $m \in A_{1}$ and $n \in A_{2}$ is $u(m, n)=1$ if $m \geqslant n$ and $u(m, n)=0$ if $m<n$. The payoffs are represented in the following matrix, where player 1 is the row player and player 2 is the column player.

\begin{tabular}{|c|cccc|}
\hline$u$ & 1 & 2 & 3 & $\ldots$ \\
\hline 1 & 1 & 0 & 0 & $\ldots$ \\
2 & 1 & 1 & 0 & $\ldots$ \\
3 & 1 & 1 & 1 & $\ldots$ \\
$\vdots$ & $\vdots$ & $\vdots$ & $\vdots$ & $\ddots$ \\
\hline
\end{tabular}




\subsubsection{Basic definitions}

Consider a zero-sum game $g(u)$ with payoff function $u$. A strategy for a player $i$ is a probability charge $\mu_{i} \in \mathcal{C}\left(A_{i}\right)$. A pair of strategies, one for each player, is called a strategy profile.

Consider a strategy profile $\left(\mu_{1}, \mu_{2}\right)$. In order to define the expected payoff for the players when they use this profile, we need a bit of notation.

A set $E \subseteq A_{1} \times A_{2}$ is called a rectangle if there are sets $E_{1} \subseteq A_{1}$ and $E_{2} \subseteq A_{2}$ with $E=E_{1} \times E_{2}$. A probability charge $\mu$ on $2^{A_{1} \times A_{2}}$ is called an extension of $\left(\mu_{1}, \mu_{2}\right)$ if for all rectangles $E=E_{1} \times E_{2}$ it holds that $\mu(E)=\mu_{1}\left(E_{1}\right) \cdot \mu_{2}\left(E_{2}\right)$. The set of extensions of $\left(\mu_{1}, \mu_{2}\right)$ is denoted by $\left\langle\mu_{1}, \mu_{2}>\right.$. ${ }^{4}$ All extensions of a given pair $\left(\mu_{1}, \mu_{2}\right)$ coincide on the algebra generated by the collection of rectangles. They may differ on other sets $E \subseteq A_{1} \times A_{2}$. Consequently, the expected payoff for the players will generally depend on the specific extension we consider. In this chapter we closely study several specific ways to extend such a strategy profile $\left(\mu_{1}, \mu_{2}\right)$.

Formally, for a given extension $\mu$ of profile $\left(\mu_{1}, \mu_{2}\right)$, the expected payoff $U(\mu)$ is defined by

$$
\mathrm{U}(\mu)=\int_{\mathrm{A}_{1} \times \mathrm{A}_{2}} \mathrm{u}(\mathrm{m}, \mathrm{n}) \mathrm{d} \mu .
$$

Thus, given a zero-sum game $\mathrm{g}(\mathrm{u})$, this construction gives rise to many ways to define a game with charges. Let each player $i$ be endowed with a function $\tau_{i}: \mathcal{C}\left(A_{1}\right) \times \mathfrak{C}\left(A_{2}\right) \rightarrow \mathcal{C}\left(A_{1} \times A_{2}\right)$. If for every profile $\left(\mu_{1}, \mu_{2}\right)$ we have that $\tau_{i}\left(\mu_{1}, \mu_{2}\right)$ is an extension of $\left(\mu_{1}, \mu_{2}\right)$, such a function is called an extension function.

For given extension functions $\tau_{1}, \tau_{2}$, the expected payoffs $U^{\tau_{1}}\left(\mu_{1}, \mu_{2}\right)$ and $\mathrm{U}^{\tau_{2}}\left(\mu_{1}, \mu_{2}\right)$ of profile $\left(\mu_{1}, \mu_{2}\right)$ are defined by

$$
\mathrm{U}^{\tau_{1}}\left(\mu_{1}, \mu_{2}\right)=\mathrm{U}\left(\tau_{1}\left(\mu_{1}, \mu_{2}\right)\right) \text { and } \mathrm{U}^{\tau_{2}}\left(\mu_{1}, \mu_{2}\right)=\mathrm{U}\left(\tau_{2}\left(\mu_{1}, \mu_{2}\right)\right) .
$$

Thus, in this chapter we record payments for player 2 instead of rewards. This way we constructed a new two-player game $G\left(\tau_{1}, \tau_{2}\right)=\left(U^{\tau_{1}},-U^{\tau_{2}}\right)$. When $\tau_{1}=\tau_{2}$, the resulting game is again a zero-sum game. But in general this need not be the case.

4 Using Axiom of Choice, it can be shown that $<\mu_{1}, \mu_{2}>$ is not empty. For more details, see Appendix 3.C. 
Example 3.3.2. Let $W$ be the winning set of a binary game. Consider a strategy profile $\left(\mu_{1}, \mu_{2}\right)$ in this game. Then, for any extension function $\tau_{1}$, the payoff for player 1 is exactly

$$
u^{\tau_{1}}\left(\mu_{1}, \mu_{2}\right)=\int_{A_{1} \times A_{2}} u(m, n) d \tau_{1}\left(\mu_{1}, \mu_{2}\right)=\tau_{1}\left(\mu_{1}, \mu_{2}\right)(W) .
$$

The payment that player 2 expects to make is $U^{\tau_{2}}\left(\mu_{1}, \mu_{2}\right)=\tau_{2}\left(\mu_{1}, \mu_{2}\right)(W)$. Hence, his utility is $-\mathrm{U}^{\tau_{2}}\left(\mu_{1}, \mu_{2}\right)$.

We will hardly ever explicitly specify the extension function in question, but simply explain the specific way extensions are constructed, and then leave it implicit how the game with charges is precisely defined.

\subsubsection{Optimistic and pessimistic players}

In this subsection we introduce two extreme cases of behavior, that depend on the attitude of the players. Our approach is based on the following lemma.

Lemma 3.3.3. Let $\mu_{1} \in \mathcal{C}\left(A_{1}\right), \mu_{2} \in \mathcal{C}\left(A_{2}\right)$ and the bounded utility function $\mathrm{u}$ be given. There exist extensions $\mu^{*}, \mu_{*} \in<\mu_{1}, \mu_{2}>$ with

$$
\mathrm{U}\left(\mu^{*}\right) \geqslant \mathrm{U}(\kappa) \text { and } \mathrm{U}\left(\mu_{*}\right) \leqslant \mathrm{U}(\kappa) \quad \text { for all } \mathrm{\kappa} \in<\mu_{1}, \mu_{2}>\text {. }
$$

Proof. We construct $\mu^{*}$. The construction of $\mu_{*}$ is similar. For every $k \in \mathbb{N}$, let $v_{k}$ denote an extension of $\left(\mu_{1}, \mu_{2}\right)$ such that $U\left(v_{k}\right) \geqslant \sup _{\kappa \in<\mu_{1}, \mu_{2}>} U(\kappa)-\frac{1}{k}$. Take any pure probability charge $\tau$ on $\mathbb{N}$. Define the probability charge $\mu^{*}$ by

$$
\mu^{*}(E)=\int_{k \in \mathbb{N}} v_{k}(E) d \tau \quad \text { for every } E \in 2^{A_{1} \times A_{2}} .
$$

Take a $k \in \mathbb{N}$ and $\kappa \in<\mu_{1}, \mu_{2}>$. We have

$$
\begin{aligned}
u\left(\mu^{*}\right) & =\int_{A_{1} \times A_{2}} u(m, n) d \mu^{*}=\int_{k \in \mathbb{N}} \int_{A_{1} \times A_{2}} u(m, n) d v_{k} d \tau \\
& =\int_{k \in \mathbb{N}} u\left(v_{k}\right) d \tau \geqslant u(k)-\frac{1}{K},
\end{aligned}
$$

where the second equality comes from Lemma 3.A.2, and the inequality is due to the fact that $\tau(\{K, K+1, \ldots\})=1$. Since $K$ was arbitrary, $U\left(\mu^{*}\right) \geqslant U(K)$.

Player 1 is optimistic if, given profile $\left(\mu_{1}, \mu_{2}\right)$, he selects an extension $\mu^{*}$ with

$$
\mathrm{U}\left(\mu^{*}\right) \geqslant \mathrm{U}(\kappa) \text { for all } \kappa \in<\mu_{1}, \mu_{2}>\text {. }
$$


Due to the previous lemma, such a selection is possible. The optimistic utility for player 1 is then defined by $U^{o}\left(\mu_{1}, \mu_{2}\right)=U\left(\mu^{*}\right)$.

In the same way, player 1 is pessimistic, if he selects an extension $\mu_{*}$ with

$$
\mathrm{U}\left(\mu_{*}\right) \leqslant \mathrm{U}(\mathrm{\kappa}) \text { for all } \mathrm{k} \in<\mu_{1}, \mu_{2}>.
$$

The pessimistic utility of player 1 is then defined by $U^{p}\left(\mu_{1}, \mu_{2}\right)=U\left(\mu_{*}\right)$.

Thus, an optimistic player 1 selects an extension $\mu^{*}$ of $\left(\mu_{1}, \mu_{2}\right)$ that, amongst all possible extensions, offers him the best expected payoff. A pessimistic player 1 selects an extension $\mu_{*}$ that offers him the worst expected payoff. However, since player 2 has to pay the amounts specified by the payoff function, an optimistic player 2 selects an extension $\mu_{*}$ that offers player 1 the worst expected payoff, and a pessimistic player 2 selects an extension $\mu^{*}$ of $\left(\mu_{1}, \mu_{2}\right)$ that, amongst all possible extensions, offers player 1 the best expected payoff. 5

\subsubsection{Double integrals}

As already mentioned in the Introduction of this chapter, a stream of literature uses expected payoffs defined via double integrals as their starting point. This type of extension of a profile $\left(\mu_{1}, \mu_{2}\right)$ is defined as follows. For every set $E \in 2^{A_{1} \times A_{2}}$, define

$$
\mu(E)=\int_{m \in A_{1}} \int_{\mathfrak{n} \in A_{2}} \mathbb{I}_{E}(m, n) d \mu_{2} d \mu_{1}, 6
$$

where $\mathbb{I}_{E}$ denotes the characteristic function of the set $E$. We define $U^{21}\left(\mu_{1}, \mu_{2}\right)$ $=\mathrm{U}(\mu)$, where 21 refers to integrating first with respect to $\mu_{2}$ and then with respect to $\mu_{1}$. We have the following basic observation.

Lemma 3.3.4. It holds that

$$
\mathrm{U}^{21}\left(\mu_{1}, \mu_{2}\right)=\int_{m \in A_{1}} \int_{n \in A_{2}} u(m, n) d \mu_{2} d \mu_{1} .
$$

5 Given a profile $\left(\mu_{1}, \mu_{2}\right)$, the optimistic payoff of player 1 is equal to the infimum of the (uniquely defined) expected payoffs from step functions, as defined in Harris et al (2005), that are larger than or equal to the payoff function for every profile of pure actions. This feature of optimistic expected payoff was pointed out to us by Ehud Lehrer. Of course a similar remark holds for pessimistic payoffs. Compare Lemma 1 of Lehrer (2009).

6 In this case we first integrate the function $\mathbb{I}_{E}(m, n)$ with respect to $\mu_{2}$. This integral will depend only on $\mathrm{m}$. Then we integrate with respect to $\mu_{1}$. 
Proof. Let $\left(\mu_{1}, \mu_{2}\right)$ be a strategy profile. Let $\mu$ be the extension of $\left(\mu_{1}, \mu_{2}\right)$ as defined above. Take $X=A_{1} \times A_{2}$, and let $S$ be the set of all simple-functions on $X$. Define for any bounded function $u$ on $X$

$$
\phi(\mathfrak{u})=\int_{(m, n) \in X} u(m, n) d \mu .
$$

Define $\psi(u)$ by

$$
\psi(u)=\int_{m \in A_{1}} \int_{\mathfrak{n} \in A_{2}} u(m, n) d \mu_{2} d \mu_{1} .
$$

It is straightforward to check that Lemma 3.A.1 applies. This completes the proof.

We can also reverse the order of integration, the resulting expected utility function is denoted by $\mathrm{U}^{12}\left(\mu_{1}, \mu_{2}\right)$.

Remark 3.3.5. Fix probability charges $\mu_{1} \in \mathcal{C}\left(A_{1}\right), \mu_{2} \in \mathcal{C}\left(A_{2}\right)$ and a bounded payoff function $u$. The Fubini-equality is

$$
\begin{aligned}
\int_{m \in A_{1}} \int_{\mathfrak{n} \in A_{2}} u(m, n) d \mu_{2} d \mu_{1} & =\int_{n \in A_{2}} \int_{m \in A_{1}} u(m, n) d \mu_{1} d \mu_{2} \\
& =\int_{A_{1} \times A_{2}} u(m, n) d \tau
\end{aligned}
$$

for any extension $\tau \in<\mu_{1}, \mu_{2}>$.

Fubini's theorem gives conditions under which the Fubini-equality holds. However, the following example shows that the Fubini-equality does not hold in general.

Example 3.3.6. Consider the Wald game again from Example 3.3.1. Take pure probability charges $\mu_{1} \in \mathcal{Q}\left(A_{1}\right)$ and $\mu_{2} \in \mathcal{Q}\left(A_{2}\right)$. In this case the Fubiniequality does not hold, since

$$
\mathrm{U}^{21}\left(\mu_{1}, \mu_{2}\right)=\int_{m \in A_{1}} 0 \mathrm{~d} \mu_{1}=0 \quad \text { while } \quad \mathrm{U}^{12}\left(\mu_{1}, \mu_{2}\right)=\int_{\mathfrak{n} \in A_{2}} 1 \mathrm{~d} \mu_{2}=1 .
$$

\subsubsection{All games are different}

Thus, a single zero-sum game may generate different expected utility functions. In this section we show that different choices for extensions can result in different games with charges. 
In general, of course, games induced by different extension functions might coincide, even though the extension functions are different. An extreme case are constant-payoff games, where all possible extension functions generate the same game. However, we have the following observation.

Proposition 3.3.7. Let $\left(\mu_{1}, \mu_{2}\right)$ be a strategy profile. Let $\mathrm{K}$ and $\mu$ be two different extensions of this profile. Then there is a zero-sum game $\mathrm{g}(\mathrm{u})$ with $\mathrm{U}(\mathrm{K}) \neq \mathrm{U}(\mu)$.

Proof. Since $k \neq \mu$, there is a set $W$ with $k(W) \neq \mu(W)$. Let $g(u)$ be the binary game with winning set $W$. Then $U(\kappa)=\kappa(W) \neq \mu(W)=U(\mu)$.

For the specific cases of $\mathrm{U}^{0}, \mathrm{U}^{\mathrm{p}}, \mathrm{U}^{21}$ and $\mathrm{U}^{12}$, we present a zero-sum game $g(u)$ for which all four extensions are different. More precisely, we present a binary game $g(u)$ and strategies $\mu_{1} \in \mathcal{C}(\mathbb{N}), \mu_{2} \in \mathcal{C}(\mathbb{N})$, and $\kappa_{1} \in \mathcal{C}(\mathbb{N})$ with $\mathrm{U}^{\mathrm{p}}\left(\mu_{1}, \mu_{2}\right)=0, \mathrm{U}^{\mathrm{o}}\left(\mu_{1}, \mu_{2}\right)=1, \mathrm{U}^{21}\left(\mu_{1}, \mu_{2}\right)=\mathrm{U}^{12}\left(\mu_{1}, \mu_{2}\right)=\frac{1}{2}, \mathrm{U}^{21}\left(\mathrm{~K}_{1}, \mu_{2}\right)=$ 1 and $\mathrm{U}^{12}\left(\mathrm{\kappa}_{1}, \mu_{2}\right)=0$. Thus, $\mathrm{U}^{\mathrm{o}}, \mathrm{U}^{\mathrm{p}}, \mathrm{U}^{21}$ and $\mathrm{U}^{12}$ define four different games, even though they are derived from the same zero-sum game.

Example 3.3.8. Consider the following binary game. Let $A_{1}=A_{2}=\mathbb{N}$. When player 1 plays $m$ and player 2 plays $n$, the payoff $u(m, n)$ is defined by

$$
u(m, n)= \begin{cases}1 & \text { if } m \geqslant n \text { and } m \text { is odd } \\ 1 & \text { if } m \leqslant n \text { and } m \text { is even } \\ 0 & \text { otherwise. }\end{cases}
$$

The game can be represented as follows.

\begin{tabular}{|c|ccccccc|}
\hline$u$ & 1 & 2 & 3 & 4 & 5 & 6 & $\ldots$ \\
\hline 1 & 1 & 0 & 0 & 0 & 0 & 0 & $\ldots$ \\
2 & 0 & 1 & 1 & 1 & 1 & 1 & $\ldots$ \\
3 & 1 & 1 & 1 & 0 & 0 & 0 & $\ldots$ \\
4 & 0 & 0 & 0 & 1 & 1 & 1 & $\ldots$ \\
5 & 1 & 1 & 1 & 1 & 1 & 0 & $\ldots$ \\
6 & 0 & 0 & 0 & 0 & 0 & 1 & $\ldots$ \\
$\vdots$ & $\vdots$ & $\vdots$ & $\vdots$ & $\vdots$ & $\vdots$ & $\vdots$ & $\ddots$ \\
\hline
\end{tabular}

Write $E=\{m \in \mathbb{N}: m$ is even $\}$ and $O=\{m \in \mathbb{N}: m$ is odd $\}$. Take a pure probability charge $\mu_{1}$ for player 1 such that

$$
\mu_{1}(E)=\mu_{1}(O)=\frac{1}{2} . \quad 7
$$

7 By Theorem 3.C.3, we can in fact construct such a pure charge. 
Let $\mu_{2}$ be an arbitrary pure probability charge for player 2 . Then for the double integrals we have

$$
\begin{aligned}
& \mathrm{U}^{12}\left(\mu_{1}, \mu_{2}\right)=\int_{n \in \mathbb{N}} \int_{m \in O} u(m, n) d \mu_{1} d \mu_{2}+\int_{n \in \mathbb{N}} \int_{m \in E} u(m, n) d \mu_{1} d \mu_{2} \\
& =\int_{n \in \mathbb{N}} \int_{\substack{m \in \mathcal{O} \\
m \geqslant n}}^{n \in \mathbb{N}} 1 \mathrm{~d} \mu_{1} \mathrm{~d} \mu_{2}+\int_{n \in \mathbb{N}} \int_{\substack{m \in \mathbb{E} \\
m>n}}^{\substack{m \in \mathbb{N} \\
m \in \mathbb{N}}} \mathrm{d} \mu_{1} \mathrm{~d} \mu_{2} \\
& =\int_{n \in \mathbb{N}} \frac{1}{2} \mathrm{~d} \mu_{2}+\int_{n \in \mathbb{N}} 0 \mathrm{~d} \mu_{2}=\frac{1}{2}+0=\frac{1}{2} \text {. }
\end{aligned}
$$

Similarly,

$$
\begin{aligned}
\mathrm{U}^{21}\left(\mu_{1}, \mu_{2}\right) & =\int_{m \in O} \int_{n \in \mathbb{N}} u(m, n) d \mu_{2} d \mu_{1}+\int_{m \in E} \int_{m \in \mathbb{N}} u(m, n) d \mu_{2} d \mu_{1} \\
& =\int_{m \in O} 0 d \mu_{1}+\int_{m \in E} 1 d \mu_{1}=0+\frac{1}{2}=\frac{1}{2} .
\end{aligned}
$$

We verify that $U^{\mathfrak{p}}\left(\mu_{1}, \mu_{2}\right)=0$. Write

$$
K=\{(m, n) \in \mathbb{N} \times \mathbb{N}: m \text { is even and } m \leqslant n\} .
$$

Define, as in Appendix 3.C, $\alpha\left(\mu_{1}, \mu_{2}\right)(K)=$

$$
\sup \left\{\sum_{i=1}^{k} \mu_{1}\left(C_{i}\right) \cdot \mu_{2}\left(D_{i}\right): C_{i} \times D_{i} \text { are mutually disjoint, contained in } K\right\} \text {. }
$$

We show that $\alpha\left(\mu_{1}, \mu_{2}\right)(K)=0$. Let $A \times B$ be a non-empty rectangle with $A \times B \subseteq K$. We show that $\mu_{1}(A) \cdot \mu_{2}(B)=0$. Suppose that $A$ is infinite. Take any $n \in B$. Since $A$ is infinite, there is $m \in A$ with $m>n$. Then $m>n$ and $(m, n) \in K$, which contradicts the definition of $K$. So, $A$ is finite. Hence, since $\mu_{1}$ is a pure charge, $\mu_{1}(A) \cdot \mu_{2}(B)=0$. It follows that $\alpha\left(\mu_{1}, \mu_{2}\right)(K)=0$.

Now define

$$
L=\{(m, n) \in \mathbb{N} \times \mathbb{N}: m \text { is odd and } m \geqslant n\} .
$$

Since also $\mu_{2}$ is a pure charge, we can show that $\alpha\left(\mu_{1}, \mu_{2}\right)(L)=0$. However, let $W$ denote the winning set of the game. Then $W=K \cup L$, so that also $\alpha\left(\mu_{1}, \mu_{2}\right)(W)=0$. The claim that $U^{p}\left(\mu_{1}, \mu_{2}\right)=0$ then follows from Proposition 3.C.5. In the same way we can verify that $U^{\mathrm{o}}\left(\mu_{1}, \mu_{2}\right)=1$.

Now, let $\mathrm{K}_{1}$ be a pure charge with $\mathrm{K}_{1}(\mathrm{E})=1$. Again, such a charge exists by Theorem 3.C.3. Then $U^{21}\left(\kappa_{1}, \mu_{2}\right)=1$ and $U^{12}\left(\kappa_{1}, \mu_{2}\right)=0$. 
Remark. The above example distinguishes our work from the work by Schervish and Seidenfeld (1996), and Capraro and Scarsini (2013). Those two articles consider convex combinations of the different orders of integration. Note that the optimistic and pessimistic approaches are not of that type. In the above example, $\mathrm{U}^{21}\left(\mu_{1}, \mu_{2}\right)=\mathrm{U}^{12}\left(\mu_{1}, \mu_{2}\right)=\frac{1}{2}$. So, convex combinations of these two payoff functions also result in a payoff of $\frac{1}{2}$. But the pessimistic and optimistic expected payoffs are different in this case, namely $\mathrm{U}^{\mathrm{p}}\left(\mu_{1}, \mu_{2}\right)=0$ and $\mathrm{U}^{\mathrm{o}}\left(\mu_{1}, \mu_{2}\right)=1$.

\subsection{GUARANTEE LEVEL}

Thus, a single zero-sum game $\mathrm{g}(\mathrm{u})$ generates many different games with charges. We study the solutions for a few of the more natural and interesting choices out of the various possibilities.

\subsubsection{Definitions}

Let $g(\mathfrak{u})$ be a zero-sum game. When $\left(\mu_{1}, \mu_{2}\right)$ has a unique extension $\mu$, we write $U\left(<\mu_{1}, \mu_{2}>\right)$ instead of $U(\mu)$. Now we turn to the definition of a guarantee level in probability charges. We first briefly discuss the definition for general extension functions. Let $\mathrm{g}(\mathfrak{u})$ be a zero-sum game, and let $\tau_{1}: \mathcal{C}\left(A_{1}\right) \times \mathcal{C}\left(A_{2}\right) \rightarrow \mathcal{C}\left(A_{1} \times A_{2}\right)$ and $\tau_{2}: \mathcal{C}\left(A_{1}\right) \times \mathcal{C}\left(A_{2}\right) \rightarrow \mathcal{C}\left(A_{1} \times A_{2}\right)$ be two extension functions. The resulting game is $\left(\mathrm{U}^{\tau_{1}},-\mathrm{U}^{\tau_{2}}\right) .{ }^{8}$

Definition 3.4.1. The $\tau_{1}$-guarantee level $v_{1}^{\tau_{1}}$ for player 1 , and the $\tau_{2}$-guarantee level $v_{2}^{\tau_{2}}$ for player 2 are defined by

$v_{1}^{\tau_{1}}=\sup _{\mu_{1} \in \mathcal{C}\left(A_{1}\right)} \inf _{\mu_{2} \in \mathcal{C}\left(A_{2}\right)} U^{\tau_{1}}\left(\mu_{1}, \mu_{2}\right)$ and $v_{2}^{\tau_{2}}=\inf _{\mu_{2} \in \mathcal{C}\left(A_{2}\right)} \sup _{\mu_{1} \in \mathcal{C}\left(A_{1}\right)} U^{\tau_{2}}\left(\mu_{1}, \mu_{2}\right)$.

If the two extension functions coincide, that is $\tau=\tau_{1}=\tau_{2}$, and $v_{1}^{\tau}=v_{2}^{\tau}$, then we say that the $\tau$-value $v^{\tau}$ of the resulting game $\left(\mathrm{U}^{\tau},-\mathrm{U}^{\tau}\right)$ exists.

In the specific cases that we mainly study-optimistic and pessimistic extensions, and double integrals—-the definitions look as follows.

$8 \overline{\text { Thus, given a strategy profile }\left(\mu_{1}, \mu_{2}\right)}$, even though the players do not need to have an agreement on the outcome of the game, we still assume that player 1 expects to receive an amount $U^{\tau_{1}}\left(\mu_{1}, \mu_{2}\right)$, while player 2 expects to pay the amount $U^{\tau_{2}}\left(\mu_{1}, \mu_{2}\right)$. 
Definition 3.4.2. The optimistic guarantee level $v_{1}^{\mathrm{o}}$ for player 1 , and the optimistic guarantee level $v_{2}^{\circ}$ for player 2 are defined by

$$
v_{1}^{o}=\sup _{\mu_{1} \in \mathcal{C}\left(A_{1}\right)} \inf _{\mu_{2} \in \mathcal{C}\left(A_{2}\right)} U^{o}\left(\mu_{1}, \mu_{2}\right) \quad \text { and } \quad v_{2}^{o}=\inf _{\mu_{2} \in \mathcal{C}\left(A_{2}\right)} \sup _{\mu_{1} \in \mathcal{C}\left(A_{1}\right)} U^{\mathcal{P}}\left(\mu_{1}, \mu_{2}\right) .
$$

The pessimistic guarantee level $v_{1}^{\text {p }}$ for player 1 , and the pessimistic guarantee level $v_{2}^{p}$ for player 2 are defined by

$$
v_{1}^{p}=\sup _{\mu_{1} \in \mathcal{C}\left(A_{1}\right)} \inf _{\mu_{2} \in \mathcal{C}\left(A_{2}\right)} U^{\mathfrak{p}}\left(\mu_{1}, \mu_{2}\right) \quad \text { and } \quad v_{2}^{p}=\inf _{\mu_{2} \in \mathcal{C}\left(A_{2}\right)} \sup _{\mu_{1} \in \mathcal{C}\left(A_{1}\right)} U^{\mathrm{o}}\left(\mu_{1}, \mu_{2}\right) .
$$

The 21-guarantee level $v_{1}^{21}$ for player 1 , and the 21-guarantee level $v_{2}^{21}$ for player 2 are defined by

$$
v_{1}^{21}=\sup _{\mu_{1} \in \mathcal{C}\left(A_{1}\right)} \inf _{\mu_{2} \in \mathcal{C}\left(A_{2}\right)} \mathrm{U}^{21}\left(\mu_{1}, \mu_{2}\right) \text { and } v_{2}^{21}=\inf _{\mu_{2} \in \mathcal{C}\left(A_{2}\right)} \sup _{\mu_{1} \in \mathcal{C}\left(A_{1}\right)} \mathrm{U}^{21}\left(\mu_{1}, \mu_{2}\right) .
$$

In the same way we can define $v_{1}^{12}$ and $v_{2}^{12}$. The fact that the pessimistic guarantee level for player 2 involves $U^{\circ}$ and the optimistic guarantee level involves $\mathrm{U}^{\mathrm{p}}$ is due to the fact that player 2 still views the amounts specified by the utility function as payments, not as rewards. 9

\subsubsection{Finitistic guarantee levels}

We also introduce several guarantee levels that do not originate from extensions of strategy profiles, but from approximations of a (zero-sum) game by finite games. Let $\mathcal{D}\left(A_{i}\right)$ be the set of probability charges (or equivalently, measures) for player $i$ with finite support. Note that $\mathcal{D}\left(A_{i}\right)$ is dense in $\mathcal{C}\left(A_{i}\right)$ with respect to the topology of weak convergence. ${ }^{10}$ We define

$$
\begin{aligned}
& v_{1}^{\mathrm{d}}=\sup _{\mu_{1} \in \mathcal{D}\left(A_{1}\right)} \inf _{\mu_{2} \in \mathcal{D}\left(A_{2}\right)} \mathrm{U}\left(<\mu_{1}, \mu_{2}>\right), \\
& v_{2}^{\mathrm{d}}=\inf _{\mu_{2} \in \mathcal{D}\left(A_{2}\right)} \sup _{\mu_{1} \in \mathcal{D}\left(A_{1}\right)} \mathrm{U}\left(<\mu_{1}, \mu_{2}>\right) .
\end{aligned}
$$

9 Note that in this chapter $v_{1}^{\mathrm{o}}, v_{2}^{\mathrm{o}}, v_{1}^{\mathrm{p}}$ and $v_{2}^{\mathrm{p}}$ are guarantee levels defined through charges. In the next chapter, we will use this notation for guarantee levels defined through countably additive probability measures, see Section 4.3 .

10 Note that this topology is not metrizable, so one needs to work with nets and not only sequences. 
For two non-empty finite sets $F_{1} \in \mathcal{F}\left(A_{1}\right)$ and $F_{2} \in \mathcal{F}\left(A_{2}\right)$, consider the game $G\left(F_{1}, F_{2}\right)$ in which player 1 is restricted to $F_{1}$ and player 2 is restricted to $F_{2}$. This game has a value, say $v\left(F_{1}, F_{2}\right)$. We define

$$
v_{1}^{f}=\sup _{F_{1} \in \mathcal{F}\left(A_{1}\right)} \inf _{F_{2} \in \mathcal{F}\left(A_{2}\right)} v\left(F_{1}, F_{2}\right) \quad \text { and } \quad v_{2}^{f}=\inf _{F_{2} \in \mathcal{F}\left(A_{2}\right)} \sup _{F_{1} \in \mathcal{F}\left(A_{1}\right)} v\left(F_{1}, F_{2}\right) .
$$

\subsubsection{The main result}

We provide the precise relationship between all guarantee levels defined above in the following theorem. The result is surprising in the sense that all guarantee levels appear to coincide, despite the fact that the games from which they are derived are, in general, all different. The proof of Theorem 3.4.3 can be found in Appendix 3.B, Theorem 3.B.2.

Theorem 3.4.3. Let $\mathrm{g}(\mathrm{u})$ be any zero-sum game. The guarantee levels of the players satisfy

$$
v_{1}^{\mathrm{f}}=v_{1}^{\mathrm{d}}=v_{1}^{\mathrm{p}}=v_{2}^{\mathrm{o}}=v_{1}^{21}=v_{2}^{21} \quad \text { and } \quad v_{2}^{\mathrm{f}}=v_{2}^{\mathrm{d}}=v_{2}^{\mathrm{p}}=v_{1}^{\mathrm{o}}=v_{2}^{12}=v_{1}^{12} \text {. }
$$

Note that, even though the double integral $U^{21}$ might differ from the pessimistic expected payoff $\mathrm{U}^{p}$, the corresponding guarantee levels for player 1 coincide. That is, $v_{1}^{21}=v_{1}^{p}$.

We identify a specific class of games for which all guarantee levels in Theorem 3.4.3 coincide. We say that a function is a simple-function when it is a finite linear combination of indicator functions of sets of the form $A \times B$, with $A \subseteq A_{1}$ and $B \subseteq A_{2}$. Harris et al (2005) call a payoff function $u: A_{1} \times A_{2} \rightarrow \mathbb{R}$ integrable when $u$ is the uniform limit of simple-functions. An integrable payoff function's expected payoff is uniquely defined for any strategy profile. Marinacci (1997) and Harris et al (2005) prove the existence of Nash-equilibrium in nonzero-sum games with integrable payoff functions.

We have the following result, which extends the classic result of von Neumann (1928).

Corollary 3.4.4. Let $\mathrm{g}(\mathrm{u})$ be a zero-sum game, where $\mathrm{u}$ is an integrable function. Then all guarantee levels are equal. 
Proof. For an integrable function, the Fubini-equality (cf. Remark 3.3.5) holds for any pair of probability charges. For a detailed proof, see Marinacci (1997), Proposition 3. This implies that $v_{1}^{21}=v_{1}^{12}$. The result is now a direct consequence of Theorem 3.4.3.

This result is also in line with the findings of Harris et al (2005). They show that, under the condition that the utility functions of an n-person normal form game are integrable, the resulting game played with charges is uniquely defined, and admits a Nash equilibrium.

\subsubsection{The classical guarantee level}

In this subsection we examine the classical guarantee levels of the players, which are defined through countably additive strategies. We restrict our attention to games where the action spaces of the players are countable. First we provide a formal definition of these classical guarantee levels, and then in Theorem 3.4.5 we relate them to the guarantee levels defined through charges and to the finitistic guarantee levels. As we show, the classical guarantee level of each player coincides with his pessimistic guarantee level.

Suppose that $A_{1}=A_{2}=\mathbb{N}$. A zero-sum game $g(\mathfrak{u})$ defined by a bounded payoff function $u: \mathbb{N} \times \mathbb{N} \rightarrow \mathbb{R}$ is called countably infinite. ${ }^{11}$ For this case, the (classical) guarantee level for player 1 and, respectively, the (classical) guarantee level for player 2 are defined as

$$
\begin{aligned}
& v_{1}=\sup _{p_{1} \in \Delta\left(A_{1}\right)} \inf _{p_{2} \in \Delta\left(A_{2}\right)} \mathrm{u}\left(<\mathrm{p}_{1}, \mathrm{p}_{2}>\right), \\
& v_{2}=\inf _{\mathrm{p}_{2} \in \Delta\left(A_{2}\right)} \sup _{p_{1} \in \Delta\left(A_{1}\right)} \mathrm{u}\left(<\mathrm{p}_{1}, \mathrm{p}_{2}>\right) .
\end{aligned}
$$

Theorem 3.4.5. Let $\mathrm{g}(\mathrm{u})$ be any countably infinite zero-sum game. The guarantee levels of the players satisfy

$v_{1}=v_{1}^{\mathrm{f}}=v_{1}^{\mathrm{d}}=v_{1}^{\mathrm{p}}=v_{2}^{\mathrm{o}}=v_{1}^{21}=v_{2}^{21}$ and $v_{2}=v_{2}^{\mathrm{f}}=v_{2}^{\mathrm{d}}=v_{2}^{\mathrm{p}}=v_{1}^{\mathrm{o}}=v_{2}^{12}=v_{1}^{12}$.

Proof. A. We show that $v_{1}^{\mathrm{d}} \leqslant v_{1}$. Take $\mu_{1} \in \mathcal{D}(\mathbb{N})$. Then

$$
\inf _{\mu_{2} \in \Delta(\mathbb{N})} U\left(<\mu_{1}, \mu_{2}>\right)=\inf _{n \in \mathbb{N}} U\left(<\mu_{1}, \delta_{n}>\right)=\inf _{\mu_{2} \in \mathcal{D}(\mathbb{N})} U\left(<\mu_{1}, \mu_{2}>\right) .
$$

11 Note that the choice of $\mathbb{N}$ is not relevant, only the cardinality matters. The results hold for any set with countable cardinality. 
Therefore,

$$
\begin{aligned}
v_{1} & =\sup _{\mu_{1} \in \Delta(\mathbb{N})} \inf _{\mu_{2} \in \Delta(\mathbb{N})} \mathrm{U}\left(<\mu_{1}, \mu_{2}>\right) \\
& \geqslant \sup _{\mu_{1} \in \mathcal{D}(\mathbb{N})} \inf _{\mu_{2} \in \Delta(\mathbb{N})} \mathrm{U}\left(<\mu_{1}, \mu_{2}>\right) \\
& =\sup _{\mu_{1} \in \mathcal{D}(\mathbb{N})} \inf _{\mu_{2} \in \mathcal{D}(\mathbb{N})} \mathrm{U}\left(<\mu_{1}, \mu_{2}>\right) \\
& =v_{1}^{\mathrm{d}} .
\end{aligned}
$$

B. We show that $v_{1} \leqslant v_{1}^{21}$. Take any $p_{1} \in \Delta(\mathbb{N})$ and any $\mu_{2} \in \mathcal{C}(\mathbb{N})$. Then by Lemma 3.A.3

$$
\begin{aligned}
\mathrm{U}^{21}\left(\mathrm{p}_{1}, \mu_{2}\right)=\mathrm{u}\left(<\mathrm{p}_{1}, \mu_{2}>\right) & =\int_{\mathfrak{n} \in \mathbb{N}} \int_{\mathfrak{m} \in \mathbb{N}} u(m, n) d p_{1} d \mu_{2} \\
& \geqslant \int_{\mathfrak{n} \in \mathbb{N}}\left(\inf _{n \in \mathbb{N}} u\left(<p_{1}, \delta_{\mathfrak{n}}>\right)\right) d \mu_{2} \\
& =\inf _{n \in \mathbb{N}} u\left(<p_{1}, \delta_{\mathfrak{n}}>\right) \\
& =\underset{\substack{\inf \\
p_{2} \in \Delta(\mathbb{N})}}{ } u\left(<p_{1}, p_{2}>\right) .
\end{aligned}
$$

So, since the last expression does not depend on $\mu_{2}$,

$$
\inf _{\mu_{2} \in \mathcal{C}(\mathbb{N})} U^{21}\left(p_{1}, \mu_{2}\right) \geqslant \inf _{p_{2} \in \Delta(\mathbb{N})} u\left(<p_{1}, p_{2}>\right) .
$$

Hence,

$$
\begin{aligned}
v_{1}^{21} & =\sup _{\mu_{1} \in \mathcal{C}(\mathbb{N})} \inf _{\mu_{2} \in \mathcal{C}(\mathbb{N})} \mathrm{U}^{21}\left(\mu_{1}, \mu_{2}\right) \\
& \geqslant \sup _{\mathrm{p}_{1} \in \Delta(\mathbb{N})} \inf _{2} \in \mathcal{C}(\mathbb{N}) \\
& \mathrm{U}^{21}\left(\mathrm{p}_{1}, \mu_{2}\right) \\
& \geqslant \sup _{\mathrm{p}_{1} \in \Delta(\mathbb{N})} \inf _{2} \in \Delta(\mathbb{N}) \\
& =v_{1} .
\end{aligned}
$$

The first half of the assertion now follows from Theorem 3.4.3. The proof of the second half is similar.

We always have $v_{1} \leqslant v_{2}$. Theorem 3.4.5 has the following corollary for the case when $v_{1}=v_{2}$ holds, that is, when the classical value exists.

Corollary 3.4.6. The classical value exists in a countably infinite zero-sum game if and only if all guarantee levels in Theorem 3.4 .5 coincide. 


\section{$3 \cdot 5$ OPTIMALITY}

When using countably additive strategies, even though the value of a game may exist, players may have only approximate optimal strategies. We investigate to which extent the use of charges guarantees existence of optimal strategies.

Let $g(u)$ be a zero-sum game, and let $\tau_{1}: \mathcal{C}\left(A_{1}\right) \times \mathcal{C}\left(A_{2}\right) \rightarrow \mathcal{C}\left(A_{1} \times A_{2}\right)$ and $\tau_{2}: \mathcal{C}\left(A_{1}\right) \times \mathcal{C}\left(A_{2}\right) \rightarrow \mathcal{C}\left(A_{1} \times A_{2}\right)$ be the extension functions employed by the respective players. Thus the resulting game is $\left(\mathrm{U}^{\tau_{1}},-\mathrm{U}^{\tau_{2}}\right)$.

Definition 3.5.1. A probability charge $\mu_{1} \in \mathcal{C}\left(A_{1}\right)$ is a $\tau_{1}$-optimal strategy for player 1 if for all strategies $\mu_{2} \in \mathcal{C}\left(A_{2}\right)$ it holds that

$$
\mathrm{u}^{\tau_{1}}\left(\mu_{1}, \mu_{2}\right) \geqslant v_{1}^{\tau_{1}} \text {. }
$$

A probability charge $\mu_{2} \in \mathcal{C}\left(A_{2}\right)$ is a $\tau_{2}$-optimal strategy for player 2 if for all strategies $\mu_{1} \in \mathcal{C}\left(A_{1}\right)$ it holds that

$$
\mathrm{u}^{\tau_{2}}\left(\mu_{1}, \mu_{2}\right) \leqslant v_{2}^{\tau_{2}}
$$

\subsubsection{Optimistic optimality}

When player 1 uses $\mathrm{U}^{\mathrm{o}}$ to evaluate outcomes, we say that an optimal strategy for player 1 is optimistic optimal. When player 2 uses $-U^{p}$ to evaluate outcomes, we say that an optimal strategy for player 2 is optimistic optimal. We have the following result.

Theorem 3.5.2. Both players have optimistic optimal strategies.

Proof. By Lemma 3.B.1, player 2 has a strategy $\sigma_{2} \in \mathcal{C}\left(A_{2}\right)$ such that for every $\mu_{1} \in \mathcal{C}\left(A_{1}\right)$ it holds that

$$
\mathrm{U}^{21}\left(\mu_{1}, \sigma_{2}\right) \leqslant v_{1}^{\mathrm{f}}
$$

Since $v_{1}^{f}=v_{2}^{\mathrm{o}}$ by Theorem 3.4.3, it follows that $\sigma_{2}$ is optimistic optimal for player 2. The proof for player 1 is similar.

Remark 3.5.3. We show how to construct optimistic optimal strategies directly. For each $k \in \mathbb{N}$, let $p_{1 k} \in \Delta\left(A_{1}\right)$ be such that, for every $p_{2} \in \Delta\left(A_{2}\right)$,

$$
\mathrm{u}\left(<\mathrm{p}_{1 \mathrm{k}}, \mathrm{p}_{2}>\right) \geqslant v-\frac{1}{\mathrm{k}} \text {. }
$$


Let $\mu_{1}$ be a pure charge. Define the probability charge $\kappa_{1}$ by

$$
\kappa_{1}(E)=\int_{k \in \mathbb{N}} p_{1 k}(E) d \mu_{1} \quad \text { for all } E \subseteq A_{1} \times A_{2} .
$$

Let $\mu_{2}$ be any probability charge of player 2 . Take a $K \in \mathbb{N}$. Then for every $n \in A_{2}$,

$$
\begin{aligned}
\int_{m \in A_{1}} u(m, n) d \kappa_{1} & =\int_{k \in \mathbb{N}} \int_{m \in A_{1}} u(m, n) d p_{1 k} d \mu_{1} \\
& =\int_{k \geqslant k} \int_{m \in A_{1}} u(m, n) d p_{1 k} d \mu_{1} \\
& \geqslant \int_{k \geqslant k}\left(v-\frac{1}{k}\right) d \mu_{1} \geqslant v-\frac{1}{k} .
\end{aligned}
$$

The first equality comes from Lemma 3.A.2, and the second equality is due to the fact that $\mu_{1}(\{K, K+1, \ldots\})=1$. Since $K$ was chosen arbitrarily, we find that

$$
\int_{m \in A_{1}} u(m, n) d \kappa_{1} \geqslant v
$$

Then for every probability charge $\mu_{2}$ of player 2 ,

$$
\mathrm{U}^{\mathrm{o}}\left(\kappa_{1}, \mu_{2}\right) \geqslant \int_{n \in A_{2}} \int_{m \in A_{1}} u(m, n) d \kappa_{1} d \mu_{2} \geqslant \int_{n \in A_{2}} v d \mu_{2}=v .
$$

Hence, $k_{1}$ is optimistic optimal for player 1 .

Example 3.5.4. Also in zero-sum games that do not have a classical value, players have optimistic optimal strategies. Consider the Wald game

\begin{tabular}{|c|cccc|}
\hline$u$ & 1 & 2 & 3 & $\ldots$ \\
\hline 1 & 1 & 0 & 0 & $\ldots$ \\
2 & 1 & 1 & 0 & $\ldots$ \\
3 & 1 & 1 & 1 & $\ldots$ \\
$\vdots$ & $\vdots$ & $\vdots$ & $\vdots$ & $\ddots$ \\
\hline
\end{tabular}

defined in Example 3.3.1. It is well-known that this game does not have a classical value, and countably additive optimal strategies do not exist.

We show that $v_{1}^{o}=1$ and that the set of optimistic optimal strategies for player 1 is exactly the set $Q(\mathbb{N})$ of pure probability charges. Take $\kappa_{1} \in \mathcal{Q}(\mathbb{N})$ and take any $\mu_{2} \in \mathcal{C}(\mathbb{N})$. We verify that $U^{o}\left(\kappa_{1}, \mu_{2}\right)=1$. Write

$$
W=\{(m, n) \in \mathbb{N} \times \mathbb{N}: m \geqslant n\} .
$$


We show that $\beta\left(\kappa_{1}, \mu_{2}\right)(K)=1$, where $\beta\left(\kappa_{1}, \mu_{2}\right)(K)=$

$$
\inf \left\{\sum_{i=1}^{k} k_{1}\left(C_{i}\right) \cdot \mu_{2}\left(D_{i}\right): C_{i} \times D_{i} \text { are mutually disjoint, and cover } K\right\}
$$

as defined in Appendix 3.C. Let $A \times B$ be a non-empty rectangle with $A \times$ $B \cap W=\emptyset$. We show that $k_{1}(A) \cdot \mu_{2}(B)=0$. Suppose that $A$ is infinite. Take any $n \in B$. Since $A$ is infinite, there is $m \in A$ with $m \geqslant n$. Then $(m, n) \in W$, which contradicts the assumption that $A \times B \cap W=\emptyset$. So, $A$ is finite. Hence, since $\mu_{1}$ is a pure charge, $\mu_{1}(A) \cdot \mu_{2}(B)=0$. It follows that $\beta\left(\kappa_{1}, \mu_{2}\right)(W)=1$. So, by Proposition 3.C. 5 there exists an extension $\mu$ of $\left(\kappa_{1}, \mu_{2}\right)$ such that $\mu(W)=1$. This implies that $U^{o}\left(\kappa_{1}, \mu_{2}\right)=1$. Since $\mu_{2} \in \mathcal{C}(\mathbb{N})$ was chosen arbitrarily, it follows that $v_{1}^{\mathrm{o}}=1$, and $\kappa_{1}$ is optimistic optimal for player 1 .

Now take $\mu_{1} \in \mathcal{C}(\mathbb{N})$ with $\mu_{1} \notin \mathcal{Q}(\mathbb{N})$. We show that $\mu_{1}$ is not optimistic optimal for player 1 . Let $\kappa_{2} \in Q(\mathbb{N})$. Write $\mu_{1}=\lambda \cdot p_{1}+(1-\lambda) \cdot \kappa_{1}$ with $p_{1} \in \Delta(\mathbb{N}), \kappa_{1} \in \mathcal{Q}(\mathbb{N})$ and $\lambda>0$. Then

$$
u^{o}\left(\mu_{1}, \kappa_{2}\right)=\lambda \cdot u^{o}\left(p_{1}, \kappa_{2}\right)+(1-\lambda) \cdot U^{o}\left(\kappa_{1}, \kappa_{2}\right)=(1-\lambda) \cdot U^{o}\left(\kappa_{1}, \kappa_{2}\right)<1 .
$$

Hence, $\mu_{1}$ is not optimistic optimal for player 1 .

Example 3.5.5. We show that an optimistic optimal strategy may be neither countably additive nor a pure probability charge, and may have to be a real mixture of the two.

Again consider the game from Example 3.3.8

\begin{tabular}{|c|ccccccc|}
\hline$u$ & 1 & 2 & 3 & 4 & 5 & 6 & $\ldots$ \\
\hline 1 & 1 & 0 & 0 & 0 & 0 & 0 & $\ldots$ \\
2 & 0 & 1 & 1 & 1 & 1 & 1 & $\ldots$ \\
3 & 1 & 1 & 1 & 0 & 0 & 0 & $\ldots$ \\
4 & 0 & 0 & 0 & 1 & 1 & 1 & $\ldots$ \\
5 & 1 & 1 & 1 & 1 & 1 & 0 & $\ldots$ \\
6 & 0 & 0 & 0 & 0 & 0 & 1 & $\ldots$ \\
$\vdots$ & $\vdots$ & $\vdots$ & $\vdots$ & $\vdots$ & $\vdots$ & $\vdots$ & $\ddots$ \\
\hline
\end{tabular}

First we show that $v_{2}^{\mathrm{o}}=\frac{1}{2}$. Suppose that player I plays the first row with probability $\frac{1}{2}$ and the second row with probability $\frac{1}{2}$. Then the expected payoff is exactly $\frac{1}{2}$ regardless of what player 2 does. So, $v_{1} \geqslant \frac{1}{2}$. 
Conversely, let $p \in \Delta(\mathbb{N})$ be any countably additive strategy for player 1 . Let $\varepsilon>0$. Take a number $m$ such that $\sum_{k=m}^{\infty} p(k)<\varepsilon$. Take $n$ with $n>m$, and let player 2 play the first column with probability $\frac{1}{2}$ and column $n$ with probability $\frac{1}{2}$. Then the expected payoff for player 1 is at most $\frac{1}{2}+\varepsilon$. These observations imply that $v_{1}=\frac{1}{2}$. Hence, by Theorem 3.4.5 also $v_{2}^{\mathrm{o}}=\frac{1}{2}$.

Now notice that for any pure probability charge $\mu_{1}$ with $\mu_{1}(O)=1$ (where $\mathrm{O}$ stands for the set of odd numbers) and for any countably additive strategy $p_{2}$, we have $\left.\mathrm{U}\left(<\mu_{1}, \mathrm{p}_{2}\right\rangle\right)=1$. Hence, countably additive strategies are not optimistic optimal for player 2 .

Next, if player 1 uses the Dirac measure $\delta_{2}$ on action 2, and player 2 uses a pure probability charge $\mu_{2}$, then $U\left(<\delta_{2}, \mu_{2}>\right)=1$. Hence, an optimistic optimal strategy for player 2 cannot be a pure probability charge either.

Consequently, any optimistic optimal strategy for player 2 must be a real mixture of a countably additive strategy and a pure probability charge. For completeness, we provide an optimistic optimal strategy for player 2. Let $\mu_{2}=\frac{1}{2} \cdot \delta_{1}+\frac{1}{2} \cdot \kappa_{2}$ where $\delta_{1}$ is the Dirac measure on action 1 and $\kappa$ is any pure probability charge on $\mathbb{N}$. Take a strategy $\mu_{1}$ of player 1 . We write $d_{1}=$ $\sum_{m \in \mathbb{N}} \mu_{1}(m)$, and $\mu_{1}=d_{1} p_{1}+\left(1-d_{1}\right) \kappa_{1}$ with $p_{1} \in \Delta(\mathbb{N})$ and $\kappa_{1} \in Q(\mathbb{N})$. Then

$$
\begin{aligned}
\mathrm{U}^{\mathrm{p}}\left(\mu_{1}, \mu_{2}\right)= & \frac{1}{2} \cdot \mathrm{d}_{1} \cdot \mathrm{U}\left(<\mathrm{p}_{1}, \delta_{1}>\right)+\frac{1}{2} \cdot\left(1-\mathrm{d}_{1}\right) \cdot \mathrm{U}\left(<\mathrm{k}_{1}, \delta_{1}>\right) \\
& +\frac{1}{2} \cdot \mathrm{d}_{1} \cdot \mathrm{U}\left(<\mathrm{p}_{1}, \mathrm{k}_{2}>\right)+\frac{1}{2} \cdot\left(1-\mathrm{d}_{1}\right) \cdot \mathrm{U}^{\mathrm{p}}\left(\mathrm{k}_{1}, \mathrm{k}_{2}\right) \\
= & \frac{1}{2} \cdot \mathrm{d}_{1} \cdot \mathrm{p}_{1}(\mathrm{O})+\frac{1}{2} \cdot\left(1-\mathrm{d}_{1}\right) \cdot \mathrm{k}_{1}(\mathrm{O}) \\
& +\frac{1}{2} \cdot \mathrm{d}_{1} \cdot \mathrm{p}_{1}(\mathrm{E})+\frac{1}{2} \cdot\left(1-\mathrm{d}_{1}\right) \cdot 0 \\
= & \frac{1}{2} \cdot \mathrm{d}_{1}+\frac{1}{2} \cdot\left(1-\mathrm{d}_{1}\right) \cdot \mathrm{k}_{1}(\mathrm{O}) \\
\leqslant & \frac{1}{2} \cdot \mathrm{d}_{1}+\frac{1}{2} \cdot\left(1-\mathrm{d}_{1}\right) \\
= & \frac{1}{2} .
\end{aligned}
$$

Here, the inequality follows from $\kappa_{1}(O) \leqslant 1$. Since $v_{2}^{o}=\frac{1}{2}$, the strategy $\mu_{2}$ is optimistic optimal for player 2. 


\subsubsection{Pessimistic optimality}

When player 1 uses $U^{p}$ to evaluate outcomes, we say that an optimal strategy for player $\mathrm{I}$ is pessimistic optimal. When player 2 uses $-\mathrm{U}^{\mathrm{O}}$ to evaluate outcomes, we say that an optimal strategy for player 2 is pessimistic optimal. Example 3.5.6. We do not know whether pessimistic optimal strategies always exist. The game below shows that the set of pessimistic optimal strategies may be non-trivial. We show that in this example pessimistic optimal strategies exist. However, countably additive strategies are not pessimistic optimal, and also not every pure charge is pessimistic optimal.

Consider the following binary game. Let $A_{1}=A_{2}=\mathbb{N}$ and define the payoff function $u$ by

$$
u(m, n)= \begin{cases}0 & \text { if } m=n \\ 1 & \text { otherwise. }\end{cases}
$$

The payoffs are displayed in the following figure.

\begin{tabular}{|c|cccc|}
\hline$u$ & 1 & 2 & 3 & $\ldots$ \\
\hline 1 & 0 & 1 & 1 & $\ldots$ \\
2 & 1 & 0 & 1 & $\ldots$ \\
3 & 1 & 1 & 0 & $\ldots$ \\
$\vdots$ & $\vdots$ & $\vdots$ & $\vdots$ & $\ddots$ \\
\hline
\end{tabular}

In this game player 1 has to hide at a certain place $m \in \mathbb{N}$. Player 2 wins if he searches at the place where player 1 is hidden, otherwise player 1 wins.

First we argue that $v_{1}^{p}=1$. Take $k \in \mathbb{N}$ and consider the countably additive strategy $p_{k}$ for player 1 such that

$$
p_{k}(m)= \begin{cases}\frac{1}{k} & \text { if } m \leqslant k \\ 0 & \text { otherwise. }\end{cases}
$$

It is clear that $\mathrm{U}\left(<\mathrm{p}_{\mathrm{k}}, \mu_{2}>\right) \geqslant \frac{k-1}{k}$ for every $\mu_{2} \in \mathcal{C}(\mathbb{N})$. Therefore $v_{1}^{p}=1$ indeed.

Now notice that no strategy $p_{1} \in \Delta(\mathbb{N})$ can be pessimistic optimal for player 1. There exists $n \in \mathbb{N}$ such that $p_{1}(n)>0$, so if player 2 plays the Dirac measure $\delta_{n}$, then $\mathrm{U}\left(<\mathrm{p}_{1}, \delta_{n}>\right)<1$. Also notice that a pure charge $\mu \in \mathcal{Q}(\mathbb{N})$ 
with $\mu(B) \in\{0,1\}$ for all $B \subseteq \mathbb{N}$ is not pessimistic optimal either, since if player 2 also plays $\mu$, then $U^{p}(<\mu, \mu>)=0$.

Now we define the strategy $\kappa_{1}$ for player 1 and show that it is pessimistic optimal. For $k \in \mathbb{N}$, and $\ell \in \mathbb{N}$ with $1 \leqslant \ell \leqslant 2^{k}$, define

$$
E_{\ell}^{k}=\left\{2^{k} \cdot n+\ell: n \in \mathbb{N} \cup\{0\}\right\} .
$$

There exists a pure charge $\mathrm{k}_{1}$ with the property that, for all $\mathrm{k}$ and $\ell, \mathrm{K}_{1}\left(\mathrm{E}_{\ell}^{\mathrm{k}}\right)=$ $\left(\frac{1}{2}\right)^{k}$. ${ }^{12}$ Suppose player 1 plays charge $\kappa_{1}$. Let $\mu_{2}$ be any charge for player 2 .

One possible cover of the diagonal is

$$
(E \times E) \cup(O \times O),
$$

where $E$ is the set of even numbers and $O$ is the set of odd numbers. This cover has a probability of $\frac{1}{2} \cdot \mu_{2}(E)+\frac{1}{2} \cdot \mu_{2}(O)=\frac{1}{2}$. In general, for fixed $k$,

$$
\bigcup_{\ell=1}^{2^{k}}\left(E_{\ell}^{k} \times E_{\ell}^{k}\right)
$$

covers the diagonal. The probability of this cover is

$$
\sum_{\ell=1}^{2^{k}}\left(\frac{1}{2}\right)^{k} \cdot \mu_{2}\left(E_{\ell}^{k}\right)=\left(\frac{1}{2}\right)^{k} .
$$

Thus, the infimum of the probabilities of these covers of the diagonal is 0 . This implies that $U^{p}\left(<\kappa_{1}, \mu_{2}>\right)=1$. Thus, $\kappa_{1}$ is pessimistic optimal for player 1 .

As a final remark, note that, since $v_{1}^{p}=1$, also $v_{1}^{o}=1$. Then, just like in the Wald game, the set $Q(\mathbb{N})$ of all pure probability charges is the set of optimistic optimal strategies for player $1 .{ }^{13}$

We do not know whether pessimistic optimal strategies always exist. For showing that optimistic optimal strategies exist, both in Theorem 3.5.2 and in

12 Since for each $k$ the collection of sets $E_{\ell}^{k}$ is a partition, it is straightforward to define $k_{1}$ on the smallest algebra containing all singletons and all sets $E_{\ell}^{k}$. The existence of such a charge $k_{1}$ defined on all subsets of $\mathbb{N}$ then follows from Theorem 3.C.3.

13 Also Capraro and Scarsini (2013) analyze this game in Example 6.9 in their paper. Even though they consider different payoff extensions, they also find that the set of optimal strategies equals the set of pure charges for this game. Additionally, Pivato (2014) in subsection 4.2 looks at a game with the same payoff function on pure action profiles, but the players' mixed strategies are nonstandard probabilities. He shows in Proposition 8 that if each player plays a uniform distribution, then this strategy profile is a Nash-equilibrium. 
Remark 3.5.3 we used an argument based on double integrals. More precisely, in both cases we used the fact that the optimistic payoff for player 1 is the same or higher than his payoff calculated through double integrals. The same argument cannot be used for pessimistic optimal strategies. We found games where pessimistic optimal strategies do exist, but we do not know whether this is the case in general for all games.

\subsubsection{Optimality for double integrals}

When player 1 (player 2) uses $\mathrm{U}^{21}$ to evaluate outcomes, we say that an optimal strategy for player 1 (player 2) is 21-optimal. The notion of 12-optimality can be defined similarly.

Theorem 3.5.7. Player 2 has 21-optimal strategies. Player 1 has 12-optimal strategies.

Proof. The first half of the assertion is an immediate consequence of Lemma 3.B.1 and the fact from Theorem 3.4.3 that $v_{1}^{f}=v_{2}^{21}$. The proof of the second half is similar.

Proposition 3.5.8. Let $\mathrm{K}_{1}$ be a pessimistic optimal strategy for player 1 . Then it is an 21-optimal strategy for player 1. Similarly, if a strategy is pessimistic optimal for player 2, then it is also 12-optimal for player 2.

Proof. The first half of the assertion is an immediate consequence of Theorem 3.4 .3 and the fact that $U^{p} \leqslant U^{21}$. The proof of the second half is similar.

\subsection{ZERO-SUM GAMES WITH CHARGES}

When both players take the same extension function, the resulting game with charges is also zero-sum. In this section we analyze when-meaning for which types of extensions_-such a resulting game has a value. The following two results are both direct consequences of Theorem 3.4.3.

Corollary 3.6.1. Suppose that one player is optimistic, and that the other player is pessimistic. Then every resulting zero-sum game with charges has a value. 
Corollary 3.6.2. Suppose that player 1 evaluates via $\mathrm{U}^{21}$ and that player 2 evaluates via $-\mathrm{U}^{21}$. Then every resulting zero-sum game with charges has a value. Similarly, there is a value, when player 1 evaluates via $\mathrm{U}^{12}$ and player 2 evaluates via $-\mathrm{U}^{12}$.

Thus, when the players agree on a given order of integration as an expected payoff, for example $\mathrm{U}^{21}$, then any zero-sum game with charges has a value. This is well-known in the literature. Heath and Sudderth (1972) also show in Theorem 3 that $v_{1}^{21}=v_{2}^{21}$.

It is tempting to think that, whenever the players agree on the extension and consequently the resulting game is zero-sum, this game automatically has a value. However, the next example shows this is not true.

Example 3.6.3. Even when the players agree on the extension function, and the resulting game with charges is zero-sum, the value of the game need not exist. Again consider the Wald game from Example 3.3.1 with payoff matrix

\begin{tabular}{|c|cccc|}
\hline$u$ & 1 & 2 & 3 & $\ldots$ \\
\hline 1 & 1 & 0 & 0 & $\ldots$ \\
2 & 1 & 1 & 0 & $\ldots$ \\
3 & 1 & 1 & 1 & $\ldots$ \\
$\vdots$ & $\vdots$ & $\vdots$ & $\vdots$ & $\ddots$ \\
\hline
\end{tabular}

We construct a total ordering on the set $\mathcal{C}(\mathbb{N})$ of probability charges on $\mathbb{N}$. Take a countable sequence $\left(\mu_{k}\right)_{k \in \mathbb{N}}$ of pure probability charges where $\mu_{k} \neq$ $\mu_{n}$ if $k \neq n$, and write $Q=\left\{\mu_{k}: k \in \mathbb{N}\right\}$. By Axiom of Choice, we can take a total ordering $\succeq$ of the set $\mathcal{C}(\mathbb{N}) \backslash Q$ of the remaining probability charges on $\mathbb{N}$. This total ordering can be extended to the set $\mathcal{C}(\mathbb{N})$ of probability charges on $\mathbb{N}$ as follows. For the elements of the sequence, if $n>k$, then $\mu_{n} \succ \mu_{k}$. For any $v \in \mathcal{C}(\mathbb{N}) \backslash Q$ and for any $n \in \mathbb{N}$ we define $\mu_{n} \succ v$. Hence, the total ordering $\succeq$ of the set $\mathcal{C}(\mathbb{N})$ is such that, for every probability charge $v \in \mathcal{C}(\mathbb{N})$, there is a pure probability charge $\mu \in \mathrm{Q}$ with $\mu \succ v$.

Next, for any pair of probability charges $\left(\mu_{1}, \mu_{2}\right)$ we define an extension $\tau\left(\mu_{1}, \mu_{2}\right)$ by letting for all $K \subseteq \mathbb{N}$

$$
\tau\left(\mu_{1}, \mu_{2}\right)(K)= \begin{cases}\int_{n \in \mathbb{N}} \int_{m \in \mathbb{N}} \mathbb{I}_{K}(m, n) d \mu_{1} d \mu_{2} & \text { if } \mu_{1} \succeq \mu_{2} \\ \int_{m \in \mathbb{N}} \int_{n \in \mathbb{N}} \mathbb{I}_{K}(m, n) d \mu_{2} d \mu_{1} & \text { if } \mu_{2} \succ \mu_{1} .\end{cases}
$$


Note that $\tau\left(\mu_{1}, \mu_{2}\right)$ is indeed an extension of $\left(\mu_{1}, \mu_{2}\right)$. When we apply this extension function to the Wald game, by Lemma 3.3.4 the resulting zero-sum game on charges is

$$
U\left(\mu_{1}, \mu_{2}\right)= \begin{cases}U^{12}\left(\mu_{1}, \mu_{2}\right) & \text { if } \mu_{1} \succeq \mu_{2} \\ U^{21}\left(\mu_{1}, \mu_{2}\right) & \text { if } \mu_{2} \succ \mu_{1} .\end{cases}
$$

Intuitively, if $\mu_{1}$ is equal to $\mu_{2}$ or $\mu_{1}$ is enumerated later than $\mu_{2}$ by $\succeq$, then the inner integral is taken with respect the strategy of player 1. As we know, this favors player 1 in the Wald game. Otherwise, the inner integral is taken with respect to the strategy of player 2 .

Take any $\mu_{1} \in \mathcal{C}(\mathbb{N})$. Then, by our construction of the total ordering $\succeq$, there is a pure charge $\mu_{2}$ such that $\mu_{2} \succ \mu_{1}$. Then $U^{\tau}\left(\mu_{1}, \mu_{2}\right)=0$. So, $\sup _{\mu_{1} \in \mathcal{C}(\mathbb{N})} \inf _{\mu_{2} \in \mathcal{C}(\mathbb{N})} U^{\tau}\left(\mu_{1}, \mu_{2}\right)=0$. Similarly, one can show that $\inf _{\mu_{2} \in \mathcal{C}(\mathbb{N})} \sup _{\mu_{1} \in \mathcal{C}(\mathbb{N})} U^{\tau}\left(\mu_{1}, \mu_{2}\right)=1$.

Thus there exists an extension $\tau\left(\mu_{1}, \mu_{2}\right)$ for each pair of probability charges $\left(\mu_{1}, \mu_{2}\right)$ with

$$
\sup _{\mu_{1} \in \mathcal{C}(\mathbb{N})} \inf _{\mu_{2} \in \mathcal{C}(\mathbb{N})} U^{\tau}\left(\mu_{1}, \mu_{2}\right)<\inf _{\mu_{2} \in \mathcal{C}(\mathbb{N})} \sup _{\mu_{1} \in \mathcal{C}(\mathbb{N})} U^{\tau}\left(\mu_{1}, \mu_{2}\right) .
$$

So, the supremum and the infimum are not interchangeable for $\tau$, and the value $v^{\tau}$ does not exist.

Example 3.6.4. On the other hand, when both players are optimistic, by Theorem 3.5.2 both players have optimal strategies. However, the resulting game need not be a zero-sum game. For example, again consider the Wald game from Example 3.3.1. Clearly, for every $p_{1} \in \Delta(\mathbb{N})$ it holds that

$$
\inf _{n \in \mathbb{N}} u\left(<p_{1}, n>\right)=0 .
$$

Hence, $v_{1}=0$. We obtain similarly that $v_{2}=1$.

\subsection{CONCLUDING REMARKS}

We studied the effect of using finitely additive probability distributions, charges, in the context of zero-sum games. Due to the ambiguity associated 
with extending strategy profiles to charges on the space of pure action profiles, the use of charges gives rise to several methods to define the guarantee level of a player.

Specifically, we studied optimistic and pessimistic guarantee levels, and guarantee levels associated with double integrals. We showed that the pessimistic guarantee level for player 1, the optimistic guarantee level for player 2, and the guarantee levels for both players in which we first integrate with respect to the strategy of the $2^{\text {nd }}$ player and then with respect to the $1^{\text {st }}$ player, all coincide. Moreover, these guarantee levels can be calculated by computing the finitistic guarantee levels.

Thus, when player 1 is optimistic, and player 2 is pessimistic, any resulting (zero-sum) game has a value. The same holds when both players use the same order of integration for the approach based on double integrals. We also proved that players always have optimal strategies with respect to the optimistic guarantee level.

In conclusion, charges are useful to define the value of an arbitrary zero-sum game, and optimistic players always have optimal strategies. It is still an open question whether pessimistic players always have optimal strategies.

In the next chapter we show that there are cases where there can be ambiguity associated also with extending strategy profiles to countably additive probabilities on the space of pure action profiles. We make further use of the optimistic and pessimistic guarantee levels, and additionally we also vary other aspects of our model.

3.A TOOLS

Lemma 3.A.1. Let $X$ be a non-empty set. Let $\mathrm{F}$ be the set of all bounded functions $\mathrm{f}: \mathrm{X} \rightarrow \mathbb{R}$. Let $\mathrm{S}$ be the set of all simple-functions in $\mathrm{F}$. Let $\phi: \mathrm{F} \rightarrow \mathbb{R}$ be a mapping such that for every $f \in F$

$$
\phi(f)=\sup \{\phi(s): s \in S \text { and } s \leqslant f\} .
$$

Let $\psi: F \rightarrow \mathbb{R}$ be linear and monotone. Suppose that $\phi(s)=\psi(s)$ for all $s \in S$. Then $\phi=\psi$. 
Proof. For every $s \in S$ and $f \in F$ with $s \leqslant f$ we have $\phi(s)=\psi(s) \leqslant \psi(f)$. Hence, for every $f \in F$

$$
\phi(f)=\sup \{\phi(s): s \in S \text { and } s \leqslant f\} \leqslant \psi(f) .
$$

Take $f \in F$. We prove $\phi(f) \geqslant \psi(f)$. Take $\varepsilon>0$. It is easy to see that there exists a simple-function $s$ such that $s \leqslant f \leqslant s+\varepsilon$, see Chapter 2. Thus

$$
\psi(f) \leqslant \psi(s+\varepsilon)=\psi(s)+\psi(\varepsilon)=\phi(s)+\varepsilon \psi(1) \leqslant \phi(f)+\varepsilon \psi(1) .
$$

Since $\varepsilon>0$ is arbitrary, $\psi(f) \leqslant \phi(f)$.

Lemma 3.A.2. Let $\mathrm{X}$ be a non-empty set. Let $\mathrm{I}$ be a set of indices. For each $\mathrm{k} \in \mathrm{I}$, let $\mu_{\mathrm{k}}$ be a probability charge on $\mathrm{X}$. Let $\tau$ be a probability charge on $\mathrm{I}$. Consider the probability charge $\sigma$ on $\mathrm{X}$ defined by

$$
\sigma(E)=\int_{k \in I} \mu_{k}(E) d \tau \quad \text { for all } \mathrm{E} \subseteq X .
$$

Then, for any bounded function $\mathrm{f}: \mathrm{X} \rightarrow \mathbb{R}$,

$$
\int_{X} f(x) d \sigma=\int_{k \in I} \int_{X} f(x) d \mu_{k} d \tau .
$$

Proof. For any simple-function $s: X \rightarrow \mathbb{R}$, where $s=\sum_{i=1}^{m} c_{i} \mathbb{I}_{F_{i}}$, we have

$$
\begin{aligned}
\int_{X} s(x) d \sigma & =\sum_{i=1}^{m} c_{i} \cdot \sigma\left(F_{i}\right)=\sum_{i=1}^{m} c_{i} \int_{k \in I} \mu_{k}\left(F_{i}\right) d \tau \\
& =\int_{k \in I} \sum_{i=1}^{m} c_{i} \mu_{k}\left(F_{i}\right) d \tau \\
& =\int_{k \in I} \int_{X} s(x) d \mu_{k} d \tau,
\end{aligned}
$$

where the first equality is based on the definition of the integral of a simplefunction and the second equality on the definition of $\sigma$. In view of Lemma 3.A.1, taking $\phi(f)=\int_{X} f(x) d \sigma$ and $\psi(f)=\int_{k \in I} \int_{X} f(x) d \mu_{k} d \tau$, the proof is complete.

For a set $A_{1}$, let $\ell\left(A_{1}\right)$ be the collection of probability charges $p_{1} \in \mathcal{C}\left(A_{1}\right)$ for which there is a sequence of points $a_{1}^{1}, a_{1}^{2}, \ldots$ in $A_{1}$ and non-negative real numbers $c^{1}, c^{2}, \ldots$ such that $p_{1}=\sum_{k} c^{k} \cdot \delta_{a_{1}^{k}}$, where $\delta_{a_{1}^{k}}$ denotes the Dirac measure on $a_{1}^{k}$. Note that automatically $\sum_{k} c^{k}=1$. We assume that all points $a_{1}^{k}$ are different. 
Lemma 3.A.3. Let $p_{1} \in \ell\left(A_{1}\right)$ and $\mu_{2} \in \mathcal{C}\left(A_{2}\right)$. Then there is a unique $\mu \in$ $\mathcal{C}\left(A_{1} \times A_{2}\right)$ such that $\mu \in<p_{1}, \mu_{2}>$. Consequently, the Fubini-equality (cf. Remark 3.3.5) holds for the pair $\left(\mathrm{p}_{1}, \mu_{2}\right)$ and any bounded payoff function.

Proof. By Proposition 3.C.5, we know that $\left\langle p_{1}, \mu_{2}\right\rangle$ is not empty. Take $\mu \in<p_{1}, \mu_{2}>$ and $k \in<p_{1}, \mu_{2}>$. Take a set $W \subseteq A_{1} \times A_{2}$. We show that $\mu(W)=\kappa(W)$.

A. We say that a set $R \subseteq A_{1} \times A_{2}$ is a rectangle if there are $B_{1} \subseteq A_{1}$ and $B_{2} \subseteq A_{2}$ such that $R=B_{1} \times B_{2}$. Observe that $\mu(R)=p_{1}\left(B_{1}\right) \cdot \mu_{2}\left(B_{2}\right)=k(R)$ for all rectangles $R$.

B. Take any $\varepsilon>0$. Take $K \in \mathbb{N}$ such that $\sum_{k>K} c^{k} \leqslant \varepsilon$. For $k \leqslant K$, define

$$
B^{k}=\left\{\left(a_{1}, a_{2}\right) \in W: a_{1}=a_{1}^{k}\right\} \text { and } B=\bigcup_{k \leqslant K} B^{k} .
$$

Notice that each $B^{k}$ is a rectangle. So, $\mu\left(B^{k}\right)=\kappa\left(B^{k}\right)$ for each $k$. Further, since all points $a_{1}^{k}$ are different, $B$ is a finite union of disjoint rectangles $B^{k}, k \leqslant K$. So, since $\mu$ and $\kappa$ are finitely additive, we also have $\mu(B)=\kappa(B)$.

C. Also define

$$
\mathrm{Z}_{1}=A_{1} \backslash\left\{\mathrm{a}_{1}^{\mathrm{k}} \in \mathrm{A}_{1}: \mathrm{k} \leqslant \mathrm{K}\right\}
$$

and $Z=Z_{1} \times A_{2}$. Then $Z$ is a rectangle, so that $\mu(Z)=p_{1}\left(Z_{1}\right) \cdot \mu_{2}\left(A_{2}\right) \leqslant$ $\varepsilon \cdot 1=\varepsilon$. In the same way we find $\kappa(Z) \leqslant \varepsilon$.

D. Now notice that $\mathrm{B} \subseteq \mathrm{W} \subseteq \mathrm{B} \cup \mathrm{Z}$, and $\mathrm{B}$ and $\mathrm{Z}$ are disjoint. So, by the additivity and monotonicity of $\mu, \mu(B) \leqslant \mu(W) \leqslant \mu(B)+\mu(Z) \leqslant \mu(B)+\varepsilon$. Hence, since $k(B)=\mu(B), \kappa(B) \leqslant \mu(W) \leqslant \kappa(B)+\varepsilon$. However, also $k(B) \leqslant$ $\kappa(W) \leqslant \kappa(B)+\varepsilon$. It follows that $\mu(W)=\kappa(W)$.

3.B PROOF OF THEOREM $3 \cdot 4 \cdot 3$

Lemma 3.B.1. Player 2 has a strategy $\sigma_{2} \in \mathfrak{C}\left(A_{2}\right)$ such that for every $\mu_{1} \in \mathfrak{C}\left(A_{1}\right)$ we have

$$
\mathrm{U}^{21}\left(\mu_{1}, \sigma_{2}\right) \leqslant v_{1}^{\mathrm{f}}
$$

Player 1 has a similar strategy $\sigma_{1}$. 
Proof. A. Take $F_{1} \in \mathcal{F}\left(A_{1}\right)$. Take $k \in \mathbb{N}$. By definition of $v_{1}^{f}$ there is a set $\mathrm{F}_{2 k} \in \mathcal{F}\left(\mathrm{A}_{2}\right)$ with

$$
v\left(\mathrm{~F}_{1}, \mathrm{~F}_{2 \mathrm{k}}\right) \leqslant v_{1}^{\mathrm{f}}+\frac{1}{\mathrm{k}}
$$

Let $p_{2 k} \in \Delta\left(F_{2 k}\right)$ be an optimal strategy for player 2 in the game $G\left(F_{1}, F_{2 k}\right)$. Naturally, $p_{2 k}$ can also be seen as an element of $\mathcal{C}\left(A_{2}\right)$.

B. Let $\lambda$ be any pure probability charge on $\mathbb{N}$. Define a strategy $\sigma_{2, \mathrm{~F}_{1}} \in$ $\mathcal{C}\left(A_{2}\right)$ for player 2 by

$$
\sigma_{2, F_{1}}(B)=\int_{k \in \mathbb{N}} p_{2 k}(B) d \lambda \quad \text { for all } B \subseteq A_{2} .
$$

C. For each $m \in F_{1}$, let $\delta_{m}$ denote the Dirac measure on $m$. We have

$$
\begin{aligned}
\mathrm{U}\left(<\delta_{\mathrm{m}}, \sigma_{2, \mathrm{~F}_{1}}>\right) & =\int_{\mathfrak{n} \in \mathrm{A}_{2}} \mathrm{u}(\mathrm{m}, \mathrm{n}) \mathrm{d} \sigma_{2, \mathrm{~F}_{1}}=\int_{\mathrm{k} \in \mathbb{N}} \int_{\mathfrak{n} \in \mathrm{A}_{2}} \mathrm{u}(\mathrm{m}, \mathrm{n}) \mathrm{d} p_{2 k} \mathrm{~d} \lambda \\
& \leqslant \int_{k \in \mathbb{N}}\left(v_{1}^{\mathrm{f}}+\frac{1}{\mathrm{k}}\right) \mathrm{d} \lambda=v_{1}^{\mathrm{f}} .
\end{aligned}
$$

Here the second equality follows from Lemma 3.A.2 and the definition of $\sigma_{2, F_{1}}$ in part B. The inequality comes from the definition of $p_{2 k}$ in part A. The last equality is a consequence of the fact that $\lambda(\{1, \ldots, K\})=0$ for every $\mathrm{K} \in \mathbb{N}$.

D. Let $\tau$ be a probability charge as in Lemma 3.C.4 on $\mathcal{F}\left(A_{1}\right)$. Thus, for every $m \in A_{1}$ we have

$$
\tau\left(\left\{F_{1} \in \mathcal{F}\left(A_{1}\right): m \in F_{1}\right\}\right)=1 .
$$

Define a strategy $\sigma_{2} \in \mathfrak{C}\left(A_{2}\right)$ for player 2 by

$$
\sigma_{2}(B)=\int_{F_{1} \in \mathcal{F}\left(A_{1}\right)} \sigma_{2, F_{1}}(B) d \tau \quad \text { for all } B \subseteq A_{2} .
$$

E. We show that for every $\mu_{1} \in \mathcal{C}\left(A_{1}\right)$

$$
\mathrm{U}^{21}\left(\mu_{1}, \sigma_{2}\right) \leqslant v_{1}^{f} .
$$

For every $m \in A_{1}$ we have

$$
\begin{aligned}
\int_{\mathfrak{n} \in A_{2}} u(m, n) d \sigma_{2} & =\int_{F_{1} \in \mathcal{F}_{1}} \int_{\mathfrak{n} \in A_{2}} u(m, n) d \sigma_{2, F_{1}} d \tau \\
& =\int_{F_{1} \in \mathcal{F}_{1}} u\left(<\delta_{m}, \sigma_{2, F_{1}}>\right) d \tau \\
& =\int_{F_{1} \in\left\{F \in \mathcal{F}_{1}: m \in F\right\}} u\left(<\delta_{m}, \sigma_{2, F_{1}}>\right) d \tau \leqslant \nu_{1}^{f} .
\end{aligned}
$$


Here the first equality follows from Lemma 3.A.2 and the definition of $\sigma_{2}$ in part $D$. The third equality holds due to our choice of $\tau$ in part $D$. In the final step we can use inequality (1) from part $A$, since now $m \in F_{1}$.

F. Since an integral is monotonic, for any $\mu_{1} \in \mathfrak{C}\left(A_{1}\right)$

$$
U^{21}\left(\mu_{1}, \sigma_{2}\right)=\int_{m \in A_{1}} \int_{n \in A_{2}} u(m, n) d \sigma_{2} d \mu_{1} \leqslant \int_{m \in A_{1}} v_{1}^{f} d \mu_{1}=v_{1}^{f} .
$$

This concludes the proof.

Theorem 3.B.2. Let $u$ be any zero-sum game. The guarantee levels of the players satisfy

$$
v_{1}^{\mathrm{f}}=v_{1}^{\mathrm{d}}=v_{1}^{\mathrm{p}}=v_{2}^{\mathrm{o}}=v_{1}^{21}=v_{2}^{21} \quad \text { and } \quad v_{2}^{\mathrm{f}}=v_{2}^{\mathrm{d}}=v_{2}^{\mathrm{p}}=v_{1}^{\mathrm{o}}=v_{2}^{12}=v_{1}^{12} .
$$

Moreover, if $\sigma_{1}$ and $\sigma_{2}$ are strategies as in Lemma 3.B.1, then $\sigma_{1}$ is optimistic optimal for player 1 , and $\sigma_{2}$ is optimistic optimal for player 2 .

Proof. First we show that $v_{1}^{\mathrm{p}} \leqslant v_{2}^{\mathrm{o}} \leqslant v_{1}^{\mathrm{f}}$. Then we show that $v_{1}^{\mathrm{f}} \leqslant v_{1}^{\mathrm{d}} \leqslant v_{1}^{\mathrm{p}} \leqslant$ $v_{1}^{21} \leqslant v_{2}^{21} \leqslant v_{1}^{\mathrm{f}}$, and that $\sigma_{2}$ is optimistic optimal for player 2 .

A. We show that $v_{1}^{\mathrm{p}} \leqslant v_{2}^{\mathrm{o}}$. It is clear from the definitions that $v_{1}^{\mathrm{p}} \leqslant v_{2}^{\mathrm{o}}$.

B. We show that $v_{2}^{\mathrm{o}} \leqslant v_{1}^{\mathrm{f}}$. Let $\sigma_{2} \in \mathcal{C}\left(A_{2}\right)$ be a strategy for player 2 as in Lemma 3.B.1. Then, for every $\mu_{1} \in \mathcal{C}\left(A_{1}\right)$ we have

$$
u^{\mathrm{p}}\left(\mu_{1}, \sigma_{2}\right) \leqslant U^{21}\left(\mu_{1}, \sigma_{2}\right) \leqslant v_{1}^{\mathrm{f}} .
$$

Therefore,

$$
v_{2}^{o}=\inf _{\mu_{2} \in \mathcal{C}\left(A_{2}\right)} \sup _{\mu_{1} \in \mathcal{C}\left(A_{1}\right)} U^{\mathfrak{p}}\left(\mu_{1}, \mu_{2}\right) \leqslant \sup _{\mu_{1} \in \mathcal{C}\left(A_{1}\right)} U^{\mathfrak{p}}\left(\mu_{1}, \sigma_{2}\right) \leqslant v_{1}^{f} .
$$

C. We show that $v_{1}^{\mathrm{f}} \leqslant v_{1}^{\mathrm{d}}$. Take $\mathrm{F}_{1} \in \mathcal{F}\left(\mathrm{A}_{1}\right)$. Then

$$
\begin{aligned}
\sup _{\mu_{1} \in \Delta\left(F_{1}\right)} \inf _{\mu_{2} \in \mathcal{D}\left(A_{2}\right)} \mathrm{U}\left(<\mu_{1}, \mu_{2}>\right) & =\sup _{\mu_{1} \in \Delta\left(F_{1}\right)} \inf _{n \in A_{2}} \mathrm{U}\left(<\mu_{1}, \delta_{n}>\right) \\
& \geqslant \inf _{F_{2} \in \mathcal{F}_{2}} \sup _{\mu_{1} \in \Delta\left(F_{1}\right)} \inf U\left(F_{2}\right. \\
& =\inf _{F_{2} \in \mathcal{F}_{2}} w\left(F_{1}, F_{2}\right) .
\end{aligned}
$$

Hence, $v_{1}^{\mathrm{d}} \geqslant v_{1}^{\mathrm{f}}$.

D. We show that $v_{1}^{\mathrm{d}} \leqslant v_{1}^{\mathrm{p}}$. Take a $p_{1} \in \mathcal{D}\left(A_{1}\right)$ and a $\mu_{2} \in \mathcal{C}\left(A_{2}\right)$. Let $\delta_{n}$ denote the Dirac measure on $n \in A_{2}$. Then

$$
\mathrm{u}\left(<\mathrm{p}_{1}, \mu_{2}>\right)=\int_{\mathfrak{n} \in \mathrm{A}_{2}} \mathrm{u}\left(<\mathrm{p}_{1}, \delta_{\mathfrak{n}}>\right) \mathrm{d} \mu_{2} \geqslant \inf _{n \in A_{2}} \mathrm{u}\left(<\mathrm{p}_{1}, \delta_{\mathfrak{n}}>\right) .
$$


We used the fact that, since $p_{1} \in \ell\left(A_{1}\right)$, the extension for $\left(p_{1}, \mu_{2}\right)$ is unique by Lemma 3.A.3. So,

$$
\inf _{\mu_{2} \in \mathcal{C}\left(A_{1}\right)} \mathrm{U}\left(<\mathrm{p}_{1}, \mu_{2}>\right) \geqslant \inf _{n \in A_{2}} \mathrm{U}\left(<\mathrm{p}_{1}, \delta_{n}>\right) \geqslant \inf _{p_{2} \in \mathcal{D}\left(A_{2}\right)} \mathrm{U}\left(<\mathrm{p}_{1}, \mathrm{p}_{2}>\right) .
$$

It follows that

$$
\sup _{p_{1} \in \mathcal{D}\left(A_{1}\right)} \inf _{\mu_{2} \in \mathcal{C}\left(A_{2}\right)} U\left(<p_{1}, \mu_{2}>\right) \geqslant \sup _{p_{1} \in \mathcal{D}\left(A_{1}\right)} \inf _{p_{2} \in \mathcal{D}\left(A_{2}\right)} U\left(<p_{1}, p_{2}>\right)=v_{1}^{d}
$$

So,

$$
v_{1}^{p}=\sup _{\mu_{1} \in \mathcal{C}\left(A_{1}\right)} \inf _{\mu_{2} \in \mathcal{C}\left(A_{2}\right)} U^{\mathrm{p}}\left(\mu_{1}, \mu_{2}\right) \geqslant \sup _{p_{1} \in \mathcal{D}\left(A_{1}\right)} \inf _{\mu_{2} \in \mathcal{C}\left(A_{2}\right)} U^{p}\left(p_{1}, \mu_{2}\right) \geqslant v_{1}^{d} .
$$

E. We show that $v_{1}^{p} \leqslant v_{1}^{21}$. Let $\mu_{1} \in \mathcal{C}\left(A_{1}\right)$ and $\mu_{2} \in \mathcal{C}\left(A_{2}\right)$ be two probability charges. We know from Lemma 3.3.4 that the probability charge $\kappa$ defined by

$$
\kappa(E)=\int_{m \in A_{1}} \int_{n \in A_{2}} \mathbb{I}_{E}(m, n) d \mu_{2} d \mu_{1} \quad \text { for all } E \subseteq \mathbb{N}
$$

is an extension of the strategy profile $\left(\mu_{1}, \mu_{2}\right)$, and that by definition $\mathrm{U}^{21}\left(\mu_{1}, \mu_{2}\right)=\mathrm{U}(\kappa)$. Hence, by definition of $\mathrm{U}^{\mathrm{p}}\left(\mu_{1}, \mu_{2}\right)$, we have $\mathrm{U}^{\mathrm{p}}\left(\mu_{1}, \mu_{2}\right) \leqslant$ $\mathrm{U}(\kappa)=\mathrm{U}^{21}\left(\mu_{1}, \mu_{2}\right)$. So,

$$
v_{1}^{p}=\sup _{\mu_{1} \in \mathcal{C}\left(A_{1}\right)} \inf _{\mu_{2} \in \mathcal{C}\left(A_{2}\right)} U^{p}\left(\mu_{1}, \mu_{2}\right) \leqslant \sup _{\mu_{1} \in \mathcal{C}\left(A_{1}\right)} \inf _{\mu_{2} \in \mathcal{C}\left(A_{2}\right)} U^{21}\left(\mu_{1}, \mu_{2}\right) \leqslant v_{1}^{21}
$$

F. We argue that $v_{1}^{21} \leqslant v_{2}^{21}$. It is clear from the definitions that $v_{1}^{21} \leqslant v_{2}^{21}$.

G. We argue that $v_{2}^{21} \leqslant v_{1}^{f}$. It follows from Lemma 3.B.1 that $v_{2}^{21} \leqslant v_{1}^{f}$.

H. Hence, $v_{1}=v_{1}^{\mathrm{f}}=v_{1}^{\mathrm{d}}=v_{1}^{\mathrm{p}}=v_{2}^{\mathrm{o}}=v_{1}^{21}=v_{2}^{21}$. It now follows from Lemma 3.B.1 that $\sigma_{2}$ is optimistic optimal for player 2 .

\section{C EXISTENCE OF CHARGES}

A collection $\mathcal{F}$ of subsets of a non-empty set $X$ is called a algebra if:

$[1] \emptyset \in \mathcal{F}$

[2] if $A \in \mathcal{F}$ then $X \backslash A \in \mathcal{F}$ and

[3] if $A_{1}, \ldots, A_{n}$ are elements of $\mathcal{F}$, then $\bigcup_{i=1}^{n} A_{i} \in \mathcal{F}$. 
The following observation can be found as 1.(ii) on page 268 in Loś and Marczewski (1949).

Lemma 3.C.1. Let $\mathcal{F}$ be an algebra on $\mathrm{X}$, and let $\mathrm{E} \subseteq \mathrm{X}$ be given. There exists a smallest algebra $\mathcal{F}(\mathrm{E})$ that contains both $\mathrm{E}$ and all elements of $\mathcal{F}$. Moreover,

$$
S \in \mathcal{F}(E) \Leftrightarrow \text { there are } A, B \in \mathcal{F} \text { with } S=(A \cap E) \cup(B \backslash E) \text {. }
$$

Proof. Suppose that $\mathcal{F} \neq 2^{X}$ and $E \notin \mathcal{F}$. It is clear that a smallest algebra $\mathcal{F}(E)$ exists that contains both $E$ and all elements of $\mathcal{F}$. It is also clear that all elements of the form $(A \cap E) \cup(B \backslash E)$ with $A, B \in \mathcal{F}$ are contained in $\mathcal{F}(E)$.

Define the set $\mathcal{G}$ by

$$
S \in \mathcal{G} \Leftrightarrow \text { there are } A, B \in \mathcal{F} \text { with } S=(A \cap E) \cup(B \backslash E) \text {. }
$$

We show that $\mathcal{G}$ is an algebra that contains both $E$ and all elements of $\mathcal{F}$. Then $\mathcal{F}(E) \subseteq \mathcal{G}$, which concludes the proof.

We show that $\mathcal{F} \subseteq \mathcal{G}$ and $E \in \mathcal{G}$. Take $\mathrm{C} \in \mathcal{F}$. Then, by choosing $\mathrm{A}=\mathrm{B}=\mathrm{C}$, we see that $C \in \mathcal{G}$. So, $\mathcal{F} \subseteq \mathcal{G}$. Further, by choosing $A=X, B=\emptyset$, we see that $E \in \mathcal{G}$.

We show that $\mathcal{G}$ is an algebra. Clearly, $\emptyset \in \mathcal{G}$. Take $S \in \mathcal{G}$. Take $A, B \in \mathcal{F}$ with $S=(A \cap E) \cup(B \backslash E)$. Then $X \backslash S=\left(A^{c} \cap E\right) \cup\left(B^{c} \backslash E\right)$. Since $\mathcal{F}$ is an algebra, we know that $A^{c}, B^{c} \in \mathcal{F}$, and we can conclude that $X \backslash S \in \mathcal{G}$.

Take $S_{1}, \ldots, S_{n} \in \mathcal{G}$. Take $A_{k}, B_{k} \in \mathcal{F}$ with $S_{k}=\left(A_{k} \cap E\right) \cup\left(B_{k} \backslash E\right)$. Then

$$
\bigcup_{k} S_{k}=\left[\left(\bigcup_{k} A_{k}\right) \cap E\right] \cup\left[\left(\bigcup_{k} B_{k}\right) \backslash E\right] \text {. }
$$

Since $\mathcal{F}$ is an algebra, $\bigcup_{k} A_{k}, \bigcup_{k} B_{k} \in \mathcal{F}$. Hence, $\bigcup_{k} S_{k} \in \mathcal{G}$.

A function $\mu: \mathcal{F} \rightarrow[0,1]$ is called a charge on $\mathcal{F}$ if $\mu(X)=1$, and $\mu(A \cup B)=$ $\mu(A)+\mu(B)$ for all disjoint sets $A, B \in \mathcal{F}$. In particular $\mu(\emptyset)=0$ for any charge $\mu$.

Take $E \subseteq X$. A charge $v$ on $\mathcal{F}(E)$ is called an extension to $\mathcal{F}(E)$ of a charge $\mu$ on $\mathcal{F}$ if $\gamma(A)=\mu(A)$ for all $A \in \mathcal{F}$. Take $Y \subset X$.

$\alpha(\mu)(Y)=\sup \{\mu(A): A \in \mathcal{F}, A \subseteq Y\}$ and $\beta(\mu)(Y)=\inf \{\mu(B): B \in \mathcal{F}, Y \subseteq B\}$.

Clearly $\alpha(\mu)(Y) \leqslant \beta(\mu)(Y)$. The following observation, which we prove for completeness, follows from Theorem 2 in Loś and Marczewski (1949). 
Lemma 3.C.2. Let $\mathcal{F}$ be an algebra, and let $\mu$ be a charge on $\mathcal{F}$. Let $\mathrm{E} \in 2^{\mathrm{X}}$ and $r \in \mathbb{R}$ with $\alpha(\mu)(E) \leqslant r \leqslant \beta(\mu)(E)$. Then there exists an extension $v$ of $\mu$ to $\mathcal{F}(E)$ such that $v(\mathrm{E})=\mathrm{r}$.

Proof. We show there exists a charge $v$ on $\mathcal{F}(E)$ with $v(A)=\mu(A)$ for all $A \in \mathcal{F}$ and $v(E)=r$.

A. We first define an extension $v$ with $v(E)=\alpha(\mu)(E)$. Define $v: \mathcal{F}(E) \rightarrow \mathbb{R}$ as follows. Take $S \in \mathcal{F}(E)$. Take $A, B \in \mathcal{F}$ with $S=(A \cap E) \cup(B \backslash E)$. Define

$$
v(S)=\alpha(\mu)(A \cap E)+\mu(B)-\alpha(\mu)(B \cap E) .
$$

Ai We show that this is a valid definition. Take $A^{\prime}, B^{\prime} \in \mathcal{F}$ with $S=$ $\left(A^{\prime} \cap E\right) \cup\left(B^{\prime} \backslash E\right)$. Then $A \cap E=A^{\prime} \cap E$. So, clearly

$$
\alpha(\mu)(A \cap E)=\alpha(\mu)\left(A^{\prime} \cap E\right) .
$$

Also $B \backslash E=B^{\prime} \backslash E$. Write $H=B \backslash B^{\prime}$ and $J=B^{\prime} \backslash B$. Then $H, J \in \mathcal{F}$. So, $\mu(B)-\mu(H)=\mu\left(B \cap B^{\prime}\right)=\mu\left(B^{\prime}\right)-\mu(J)$. Hence, it remains to show that

$$
\alpha(\mu)(B \cap E)-\mu(H)=\alpha(\mu)\left(B^{\prime} \cap E\right)-\mu(J) .
$$

Take $\mathrm{D} \in \mathcal{F}$ with $\mathrm{D} \subseteq \mathrm{B} \cap \mathrm{E}$. Write $\mathrm{G}=\mathrm{D} \backslash \mathrm{H}$. Then $\mathrm{G} \cup \mathrm{J} \in \mathcal{F}$, and $\mathrm{G} \cup \mathrm{J} \subseteq$ $B^{\prime} \cap$ E. So,

$$
\begin{aligned}
\mu(D)-\mu(H) & \leqslant \mu(G \cup H)-\mu(H) \\
& =\mu(G)+\mu(H)-\mu(H) \\
& =\mu(G)+\mu(J)-\mu(J) \\
& =\mu(G \cup J)-\mu(J) \\
& \leqslant \alpha(\mu)\left(B^{\prime} \cap E\right)-\mu(J) .
\end{aligned}
$$

This shows that

$$
\alpha(\mu)(B \cap E)-\mu(H) \leqslant \alpha(\mu)\left(B^{\prime} \cap E\right)-\mu(J) .
$$

The reverse inequality follows similarly. This shows that the definition of $v$ is sound.

A2. Take $F \in \mathcal{F}$. We show that $v(F)=\mu(F)$. Take $A=B=F$. Then

$$
v(F)=\alpha(\mu)(A \cap E)+\mu(B)-\alpha(\mu)(B \cap E)=\mu(B)=\mu(F) .
$$


We show that $\gamma(E)=\alpha(\mu)(E)$. Take $A=X$ and $B=\emptyset$. Then

$$
v(E)=\alpha(\mu)(X \cap E)+\mu(\emptyset)-\alpha(\mu)(\emptyset \cap E)=\alpha(\mu)(E) .
$$

A3. Take two sets $A$ and $B$ in $\mathcal{F}$ for which $A \cap E$ and $B \cap E$ are disjoint. We show that $\alpha(\mu)(A \cap E)+\alpha(\mu)(B \cap E)=\alpha(\mu)((A \cup B) \cap E)$. It is clear that $\alpha(\mu)(A \cap E)+\alpha(\mu)(B \cap E) \leqslant \alpha(\mu)((A \cup B) \cap E)$. We show the reverse inequality. Take a $C \in \mathcal{F}$ with $C \subseteq(A \cup B) \cap E)$. Define $U=C \cap A$ and $V=C \cap B$. Then $\mathrm{U}, \mathrm{V} \in \mathcal{F}, \mathrm{U} \cap \mathrm{V}=\emptyset$, and $\mathrm{C}=\mathrm{U} \cup \mathrm{V}$. So,

$$
\mu(C)=\mu(U)+\mu(V) \leqslant \alpha(\mu)(A \cap E)+\alpha(\mu)(B \cap E) .
$$

This completes the proof.

A4. We show that $v$ is additive on $\mathcal{F}(E)$. Take two disjoint sets $S$ and $T$ in $\mathcal{F}(E)$. We show that $v(S)+v(T)=v(S \cup T)$. Take sets $A, B, C, D \in \mathcal{F}$ such that $S=(A \cap E) \cup(B \backslash E)$ and $T=(C \cap E) \cup(D \backslash E)$. First note that

$$
S \cup T=((A \cup C) \cap E) \cup((B \cup D) \backslash E) .
$$

Note that $A \cap E$ and $C \cap E$ are disjoint. Moreover, $B$ and $D$ can be chosen in such a way that $B \cap D=\emptyset$. Then, using $\mathbf{A}_{3}$,

$$
\begin{aligned}
v(S)+v(T)= & \alpha(\mu)(A \cap E)+\mu(B)-\alpha(B \cap E) \\
& +\alpha(\mu)(C \cap E)+\mu(D)-\alpha(D \cap E) \\
= & \alpha(\mu)((A \cup C) \cap E)+\mu(B \cup D)-\alpha((B \cup D) \cap E) \\
= & v(S \cup T) .
\end{aligned}
$$

B. In the same way we can construct $\mathrm{K}$ on $\mathcal{G}$ with $\mathrm{k}(\mathrm{E})=\beta(\mu)(E)$. Taking a convex combination shows that we can find a $v$ on $\mathcal{G}$ with $v(E)=r$.

Let $2^{X}$ denote the collection of subsets of $X$. Note that $2^{X}$ is an algebra. Let $\mu$ be a charge on $\mathcal{F}$. An extension of $\mu$ is a charge $v$ on $2^{X}$ with $\gamma(A)=\mu(A)$ for all $A \in \mathcal{F}$. Let $\mu$ be an extension of $\left(\mu_{1}, \mu_{2}\right)$. By monotonicity of $\mu$,

$$
\alpha(\mu)(E) \leqslant \mu(E) \leqslant \beta(\mu)(E)
$$

for every set $E \in 2^{X \times X}$.

Theorem 3.C.3. Assume Axiom of Choice. Let $\mathcal{F}$ be an algebra, and let $\mu$ be a charge on $\mathcal{F}$. Let $E \in 2^{X}$ and $r \in \mathbb{R}$ with $\alpha(\mu)(E) \leqslant r \leqslant \beta(\mu)(E)$. Then there exists an extension $v$ of $\mu$ such that $v(E)=r$. 
Proof. The statement now follows from Lemma 3.C.2 and the Lemma of Zorn.

Corollary 3.C.4. Let $\Omega$ be a non-empty set. Let $\mathcal{F}$ denote the collection of all finite subsets of $\Omega$. Then there is a probability charge $\tau$ on $\mathcal{F}$ such that for every $\omega \in \Omega$

$$
\tau(\{F \in \mathcal{F}: \omega \in F\})=1 .
$$

Proof. Take F, G $\in$ F. Define

$$
V[F, G]=\{H \in \mathcal{F}: F \subseteq H \text { and } G \cap H=\emptyset\} .
$$

Let $\mathcal{V}$ be the collection of all finite unions of such sets $V[F, G]$. This collection is an algebra. Moreover, define $\tau$ on $\mathcal{V}$ as the linear extension of

$$
\tau(\mathrm{V}[\mathrm{F}, \mathrm{G}])= \begin{cases}1 & \text { if } \mathrm{G}=\emptyset \\ 0 & \text { otherwise. }\end{cases}
$$

Then $\tau$ is a probability charge on $\mathcal{V}$. The result now follows directly from Theorem 3.C.3.

For probability charges $\mu_{1} \in \mathcal{C}\left(A_{1}\right)$ and $\mu_{2} \in \mathcal{C}\left(A_{2}\right)$ and a set $E \in 2^{A_{1} \times A_{2}}$, we define $\alpha\left(\mu_{1}, \mu_{2}\right)(E)=$

$$
\sup \left\{\sum_{i=1}^{k} \mu_{1}\left(C_{i}\right) \cdot \mu_{2}\left(D_{i}\right): C_{i} \times D_{i} \text { are mutually disjoint, contained in } E\right\},
$$

and $\beta\left(\mu_{1}, \mu_{2}\right)(E)=$

$$
\inf \left\{\sum_{i=1}^{k} \mu_{1}\left(C_{i}\right) \cdot \mu_{2}\left(D_{i}\right): C_{i} \times D_{i} \text { are mutually disjoint, and cover } E\right\} .
$$

Clearly, $\alpha\left(\mu_{1}, \mu_{2}\right)(E) \leqslant \beta\left(\mu_{1}, \mu_{2}\right)(E)$. Let $\mu$ be an extension of $\left(\mu_{1}, \mu_{2}\right)$. By monotonicity of $\mu$,

$$
\alpha\left(\mu_{1}, \mu_{2}\right)(E) \leqslant \mu(E) \leqslant \beta\left(\mu_{1}, \mu_{2}\right)(E)
$$

for every set $E \in 2^{A_{1} \times A_{2}}$.

Proposition 3.C.5. Let $\mu_{1} \in \mathcal{C}\left(A_{1}\right)$ and $\mu_{2} \in \mathcal{C}\left(A_{2}\right)$. Let $E \in 2^{A_{1} \times A_{2}}$ and $r \in \mathbb{R}$ with $\alpha\left(\mu_{1}, \mu_{2}\right)(E) \leqslant r \leqslant \beta\left(\mu_{1}, \mu_{2}\right)(E)$. Then there exists an extension $\mu$ of $\left(\mu_{1}, \mu_{2}\right)$ such that $\mu(E)=r$. 


\section{CATCH GAMES - THE IMPACT OF MODELING DECISIONS}

\subsection{INTRODUCTION}

In the previous chapter we considered a fairly large set of zero-sum games. This chapter is a study of catch games, a class of two-person zero sum games with infinite action spaces. We use this class of games to highlight the effects of modeling decisions on the guarantee levels of the players, and hence on the existence of the value of the game. Catch games and similar games are used for example to compute optimal strategies in games of pursuit, or to study tactics of evasion. Besides that, modeling questions similar to the ones in catch games can arise in any economic model where the players' action spaces are assumed to be infinite.

CAtch Games A catch game is a game played by two players. Player $I$ chooses an action of a given infinite set $A$ of actions. An action $a \in A$ is interpreted as a location for player 1 to hide. Player 2 simultaneously selects a finite set $\mathrm{b} \subseteq \mathrm{A}$ of possible locations. The interpretation is that player 2 checks finitely many possible locations for the presence of player 1 . The collection of actions of player 2 is denoted by B. Player 1 wins the game if his choice $a \in A$ of location is not an element of the finite set $b \in B$ of locations that player 2 selects to check. Thus, player 1 wins if $a \notin b$. Otherwise, when $a \in b$, player 1 gets caught, and player 2 wins the game. Notice that the game is entirely specified by the choice of the action set $A$ of player 1 .

Players are assumed to use mixed strategies. In order to model this assumption, each of the sets $A$ and $B$ is endowed with a sigma-algebra, $\mathcal{F}(A)$ and $\mathcal{F}(B)$, respectively. Player 1 selects an element of the set of probability measures on $\mathcal{F}(A)$, and player 2 selects an element of the set of probability measures on $\mathcal{F}(B)$. The result of these choices is evaluated by means of computation of expected payoffs, which is identical to the probability of success, with 
respect to outer measure. The main questions in this chapter revolve around the possible set of mixed strategies and the corresponding guarantee levels, which are calculated through the expected payoffs.

optimistic Versus pessimistic eValuation In this context, we consider two possible attitudes for each player. An optimistic player 1 maximizes the probability that $a \notin b$, i.e. that player 1 avoids player 2. A pessimistic player 1 minimizes the probability that $a \in b$, i.e. that player 2 catches player 1.

Since we compute outer measures, these two attitudes are not identical. It may happen that in a catch game an optimistic player 1 thinks he can successfully, with probability 1 , avoid player 2 , while in the same catch game a pessimistic player 1 thinks that, with probability 1 , player 2 is able to catch player 1 .

The Central Results Our first central result, Theorem 4.4.1, states that player 2, both in the optimistic and the pessimistic case, thinks that player 1 will win the game. Our central result for player 1, Theorem 4.4.2, considers three exhaustive and mutually exclusive cases.

Case [1]. In this case $\mathcal{F}(A)$ admits only trivial probability measures. Player 1 , both in the optimistic and in the pessimistic case, thinks that player 2 will win the game. Consequently, the game does not have a value.

Case [2]. In this case, there exists a non-trivial probability measure on $\mathcal{F}(A)$ that does not have any atoms. Player 1 , both in the optimistic and in the pessimistic case, thinks that player 1 will win the game. Consequently, the value of the game is 1 .

Case [3]. In this case there exist non-trivial probability measures on $\mathcal{F}(A)$, but each such measure has an atom. The optimistic player 1 thinks that he will win the game, so that the value of the game is 1 .

On the remaining situation, case [3], and player 1 being pessimistic, we only have a partial result, Theorem 4.4.6. We have an example of sigma-algebras $\mathcal{F}(A)$ and $\mathcal{F}(B)$ that fall within the context of case [3] in which a pessimistic player 1 thinks that player 2 will win the game. Thus, here a pessimistic player 1 disagrees with the optimistic player 1 . 
Among the three cases and the two different attitudes not only the guarantee levels of player 1 vary, but also his sets of optimal strategies are quite different.

THE CONSEQUENCES The modeling decisions we study affect the collection of mixed strategies available to the players, and hence have influence on which case is realized. The decisions we vary are as follows.

[1] The choice of sigma-algebra on which mixed strategies are defined.

[2] Optimistic versus pessimistic players.

[3] The set theoretic setting in which we execute our analysis.

Each of these decisions determines in which of the three above cases we end up. Thus, the guarantee levels change when we change our modeling decision. Apart from these modeling decisions, which take place in the setting of countably additive strategies, we also analyze the effect on the guarantee levels of the players when the players use finitely additive strategies instead of countably additive strategies. Thus, the fourth modeling decision we vary is as follows.

[4] Countably additive strategies versus finitely additive strategies.

We first briefly discuss the possible effects of each modeling decision, under the assumption that players use countably additive strategies.

[1] Different sigma-algebras may allow different types of strategies. For example, in the partial result in case [3], the only non-trivial probability measures that the sigma-algebra $\mathcal{F}(A)$ allows are atomic. In this case, a pessimistic player 1 thinks player 2 wins the game. However, in the case where $A=[0,1]$, we can extend $\mathcal{F}(A)$ to the Borel sigma-algebra $\mathcal{B}(A)$. Since the Lebesgue measure is an atomless probability measure on $\mathcal{B}(A)$, that would put us in case [2], where a pessimistic player 1 thinks he wins the game.

[2] The attitude (pessimistic or optimistic) of player 1 affects the guarantee level of player 1 , and thus the existence of the value, in case [3].

[3] The set theoretic setting may also affect which case we end up in. For each of the three cases, we have a collection of set theoretic axioms that force the game to be in that specific case. We will provide an example where the 
action set $A=[0,1]$ and the sigma algebra $\mathcal{F}(A)$ is its power set. This is a rather natural setting and even so, player $I^{\prime}$ s guarantee levels can be quite different, 0 or 1 , depending on the assumed set theoretic axioms. (see Section $4 \cdot 5)$

[4] Finally, we study the case where players use finitely additive probabilities as mixed strategies, under the assumption that both have the power set as sigma-algebra. Both the guarantee level of a pessimistic player 1 and the guarantee level of an optimistic player 2 are equal to zero. However, the guarantee levels of an optimistic player 1 and a pessimistic player 2 are both equal to one. Thus, when one player is optimistic, and the other is pessimistic, the value of the game exists. Evidently, these results are in stark contrast to the countably additive case discussed earlier. (see Section 4.6)

STRUCTURE OF THE CHAPTER In Section 4.2 we discuss some preliminaries. In Section 4.3 we define catch games and the solution concepts that we study. In Section 4.4, we present our main results. The proofs are given in Appendix 4.A. In Section 4.5 we show how set theoretic assumptions affect our results, and provide some examples to illustrate the different cases in our main theorem. In Section 4.6 we report our results for the finitely additive setup, and compare those to the countably additive setup. In Section $4 \cdot 7$ we briefly argue that our requirements on the sigma-algebras $\mathcal{F}(A)$ and $\mathcal{F}(B)$ are fairly mild.

RELATED LITERATURE Instances of catch games have previously been considered-mainly as illustrative examples—see Example 3.5.6, Capraro and Scarsini (2013) and Pivato (2014). A related line of literature is on hideand-seek games, which often have a dynamic flavor where the seeker has to develop a search strategy to detect the hider. Applications of hide-andseek games can be found in for example Crawford and Iriberri (2007) and Rubinstein-Tversky (1996). Other classes of related games are for example search games (Isaacs (1965)), Stackelberg security games (Kiekintveld et al (2009)) and patrolling games (Abaffy et al (2014)).

Interplay between set theory and game theory Our paper is not the first paper where game theory and set theory interact. Set theory plays an important role in several game theoretic papers. For example, Prikry and Sudderth 
(2016) derive properties of the guarantee levels in certain zero sum games from descriptive set theory. Zame (2007) also investigated the relationship between economic theory and set theory, when he showed that the existence of an ethical preference relation is independent of a certain collection of set theoretic axioms. An ethical preference relation is a formalisation of equal regard of all individuals in an intergenerational model. This model can also be interpreted as a repeated game. Peters and Vermeulen (2012) show that the set of bargaining solutions satisfying a certain collection of properties differs when assuming the Axiom of Determinacy compared to assuming the Axiom of Choice.

Conversely, there is a line of research where game theory is used for set theoretic purposes. For example, the determinacy of Gale-Stewart games (Gale and Stewart (1953)) has implications in set theory, see Kechris (1995), p. 138139 and Martin (1975). Similarly, topological games such as Banach-Mazur games, Oxtoby (1980), p. 27, also have applications in set theory.

Finite additivity For related literature on finitely additivity probabilities, see Chapter 1. In addition, for related literature on finitely additive strategies in games, see Chapter 3 .

\subsection{PRELIMINARIES}

For a set $A$, we write $2^{A}$ to denote the collection of all subsets of $A$. For a finite set $A,|A|$ denotes the number of elements of $A$.

Let $S$ be a non-empty set and let $\mathcal{F}$ denote a sigma-algebra on $S$. A function $p: \mathcal{F} \rightarrow[0, \infty)$ is called countably additive if for all collections $\left\{\mathrm{E}_{i}\right\}_{i=1}^{\infty}$ of pairwise disjoint sets in $\mathcal{F}$ it holds that

$$
p\left(\bigcup_{i=1}^{\infty} E_{i}\right)=\sum_{i=1}^{\infty} p\left(E_{i}\right) .
$$

A measure on $S$ is a countably additive function $p$ such that $p(S)>0$. A measure $p$ is called a probability measure if $p(S)=1$. We denote by $\Delta(S)$ the set of all probability measures on $S$. Let $p \in \Delta(S)$. A set $A \in \mathcal{F}$ is called an atom of $p$ if

$$
\text { [1] } p(A)>0 \text {, and }
$$


[2] there is no set $B \in \mathcal{F}$ such that $B \subset A$ with $0<p(B)<p(A)$.

If a measure has no atoms, then it is called atomless. Suppose that $\{s\} \in \mathcal{F}$ for every $s \in S$. A measure $p$ is called non-trivial if $p(\{s\})=0$ for every $s \in S$. Note that an atomless measure is non-trivial.

Consider two non-empty sets $S$ and $T$. Let $\mathcal{F}(S)$ be a sigma-algebra on $S$ and let $\mathcal{F}(T)$ be a sigma-algebra on $T$. A set $Z \subseteq S \times T$ is called a rectangle if there are sets $A \in \mathcal{F}(S)$ and $B \in \mathcal{F}(T)$ such that $Z=A \times B$. We write $Z_{1}=A$ and $\mathrm{Z}_{2}=\mathrm{B}$.

A collection $\left\{Z^{i}\right\}_{i=1}^{\infty}$ is a cover of $X \subseteq S \times T$ if

[1] for each $i \in \mathbb{N}$, the set $Z^{i}$ is a rectangle in $S \times T$, and

[2] $X \subseteq \bigcup_{i=1}^{\infty} Z^{i}$.

Let $p_{1} \in \Delta(S), p_{2} \in \Delta(T)$ and $X \subseteq S \times T$. The outer measure $<p_{1}, p_{2}>(X)$ of $X$ with respect to $\left(p_{1}, p_{2}\right)$ is defined by

$$
<p_{1}, p_{2}>(X)=\inf \left\{\sum_{i=1}^{\infty} p_{1}\left(Z_{1}^{i}\right) \cdot p_{2}\left(Z_{2}^{i}\right):\left\{Z^{i}\right\}_{i=1}^{\infty} \text { is a cover of } X\right\} .
$$

Note that for a rectangle $Z \subseteq S \times T$ we have $<p_{1}, p_{2}>(Z)=p_{1}\left(Z_{1}\right) \cdot p_{2}\left(Z_{2}\right)$. In this chapter we do not always assume the Axiom of Choice, and when we do, we explicitly mention it.

\section{3 САTCH GAMES}

This chapter is a study of catch games. A catch game is defined as follows. Let $A$ be an infinite set, and let $B$ be the set consisting of all finite subsets of $A$. Consider the zero-sum game in which player 1 chooses an action a from $A$, and simultaneously, player 2 chooses an action $b$ from $B$. The payoff is 0 if $a \in b$, i.e. when player 2 catches player 1 , and 1 if $a \notin b$. Player 2 pays the payoff to player 1 . Thus, the winning set of player 1 in a catch game is

$$
W^{1}=\{(a, b) \in A \times B: a \notin b\} .
$$

Similarly $W^{2}=\{(a, b) \in A \times B: a \in b\}$ is the winning set for player 2. ${ }^{1}$ Note that a catch game is a type of binary game, as defined in Section 3.3.

1 When $A$ is finite, the whole set $A$ is an action for player 2, and he can win by choosing this action. Therefore we restrict our attention to cases where $A$ is infinite. 
SIGMA-ALGEBRAS We endow $A$ and $B$ with sigma-algebras $\mathcal{F}(A)$ and $\mathcal{F}(B)$, respectively. For our results we need various minimum requirements on $\mathcal{F}(A)$ and $\mathcal{F}(B)$. Therefore we first briefly discuss the requirements we need. For the interested reader, in Section 4.7 we discuss our requirements on the sigmaalgebras $\mathcal{F}(A)$ and $\mathcal{F}(B)$ in more detail, and argue that our conditions are relatively mild, in the sense that they allow for many different sigma-algebras.

Condition [A] For every $a \in A,\{a\} \in \mathcal{F}(A)$.

We explain the condition on $\mathcal{F}(B)$ we use in the chapter. For any $m \in \mathbb{N}$, let $B_{m}$ be the set of actions $b$ for player 2 with $|b|=m$. Let $I$ be a finite subset of $A$, and let $m \in \mathbb{N}$. We define

$$
\mathrm{Q}(\mathrm{I}, \mathrm{m})=\left\{\mathrm{b} \in \mathrm{B}_{\mathrm{m}}: \mathrm{I} \text { is a subset of } \mathrm{b}\right\} .
$$

Condition $[\mathrm{B}] \quad$ For any choice of $\mathrm{I}$ and $\mathrm{m}, \mathrm{Q}(\mathrm{I}, \mathrm{m}) \in \mathcal{F}(\mathrm{B})$.

Condition [B] has the following basic, but useful, consequences.

[B1] For every $m, B_{m} \in \mathcal{F}(B)$. To see this, take $I=\emptyset$.

[B2] For every $a \in A, B(a)=\{b \in B: a \in b\} \in \mathcal{F}(B)$. To see this, take $I=\{a\}$ and any $m \in \mathbb{N}$. Then $Q(I, m)=\left\{b \in B_{m}: a \in b\right\}$. Hence, $B(a)$ is the union over all $m$ of the sets $Q(I, m)$.

[B3] For every $c \in B,\{c\} \in \mathcal{F}(B)$. To see this, take $I=c$ and $m=|c|$. Then, for an arbitrary $b \in B$, we have that $b \in Q(I, m)$ precisely when $b$ is a set with $|c|$ elements that contains $c$. This implies that $b=c$, so that $\mathrm{Q}(\mathrm{I}, \mathrm{m})=\{\mathrm{c}\}$. Hence, $\{\mathrm{c}\} \in \mathcal{F}(\mathrm{B})$.

Conditions [A] and [B] are assumed throughout this chapter. Therefore, we do not mention their validity explicitly. Notice that $\left[\mathrm{B}_{1}\right]$ and $\left[\mathrm{B}_{2}\right]$ together are equivalent to $[B]$, as $Q(\emptyset, m)=B_{m}$, and

$$
\mathrm{Q}(\mathrm{I}, \mathrm{m})=\mathrm{B}_{\mathrm{m}} \bigcap\left[\bigcap_{\mathrm{a} \in \mathrm{I}} \mathrm{B}(\mathrm{a})\right] .
$$

We sometimes need a stronger condition on $\mathcal{F}(B)$. Let $D$ be any subset of $A$, and let $k \in \mathbb{N} \cup\{0\}$. We define

$$
Y(D, k)=\{b \in B: D \cap b \text { has exactly } k \text { elements }\}
$$


Condition $[C]$ For any choice of $D \in \mathcal{F}(A)$, and any $k, Y(D, k) \in \mathcal{F}(B)$.

Notice that condition $[\mathrm{C}]$ implies condition [B]. To see this, first notice that $Y(A, m)=B_{m}$. Hence, for any finite $I$ and $m \in \mathbb{N}, Q(I, m)=Y(I,|I|) \cap$ $Y(A, m)$.

GUARANTEE LEVELS We examine 4 different guarantee levels for a catch game. Let $\mathcal{F}(A)$ and $\mathcal{F}(B)$ be fixed. The set of mixed strategies for player 1 is $\Delta(A)$, and the set of mixed strategies for player 2 is $\Delta(B)$. Write $p=\left\langle p_{1}, p_{2}\right\rangle$ for the outer measure defined by $p_{1}$ and $p_{2}$. We define

$$
\begin{aligned}
v_{1}^{o} & :=\sup _{p_{1} \in \Delta(A)} \inf _{p_{2} \in \Delta(B)} p\left(W^{1}\right) \\
v_{1}^{p} & :=\sup _{p_{1} \in \Delta(A)} \inf _{p_{2} \in \Delta(B)}\left[1-p\left(W^{2}\right)\right] \\
v_{2}^{p} & :=\inf _{p_{2} \in \Delta(B)} \sup _{p_{1} \in \Delta(A)} p\left(W^{1}\right) \\
v_{2}^{o} & :=\inf _{p_{2} \in \Delta(B)} \sup _{p_{1} \in \Delta(A)}\left[1-p\left(W^{2}\right)\right] .
\end{aligned}
$$

In the definition of these guarantee levels the superscript $p$ stands for pessimistic and superscript o stands for optimistic. ${ }^{2}$ The subscript in the notation for these levels indicates which player is under consideration. Thus subscript 1 indicates guarantee levels for the payoff to player 1 , and subscript 2 indicates guarantee levels for the payoff of player 2. ${ }^{3}$

Remarks. A few comments on these definitions are in order here.

[1] Notice that $W^{1} \cup W^{2}=A \times B$. So, it follows immediately from the definition of outer measure that

$$
<\mathrm{p}_{1}, \mathrm{p}_{2}>\left(\mathrm{W}^{1}\right)+<\mathrm{p}_{1}, \mathrm{p}_{2}>\left(\mathrm{W}^{2}\right) \geqslant 1
$$

for any $p_{1} \in \Delta(A)$ and $p_{2} \in \Delta(B)$. Hence, $v_{1}^{o} \geqslant v_{1}^{p}$ and $v_{2}^{p} \geqslant v_{2}^{o}$.

[2] Let $\mathcal{F}(A \times B)$ denote the sigma-algebra induced by the rectangles of the product space $A \times B$. Based on Carathéodory's extension theorem, any two probability measures $p_{1} \in \Delta(A)$ and $p_{2} \in \Delta(B)$ generate

2 The guarantee level $v_{1}^{\mathrm{o}}$ is considered to be a more optimistic assessment by player 1 than $v_{1}^{\mathrm{p}}$ because $v_{1}^{\mathrm{o}} \geqslant v_{1}^{\mathrm{p}}$.

3 Note that in this chapter $v_{1}^{\mathrm{o}}, v_{1}^{\mathrm{p}}, v_{2}^{\mathrm{p}}$ and $v_{2}^{\mathrm{o}}$ are defined through countably additive strategies, while in the previous chapter this notation was used for guarantee levels defined through finitely additive strategies, see Definition 3.4.2. 
a unique probability measure $p$ over elements of $\mathcal{F}(A \times B)$. Hence, if $W^{1} \in \mathcal{F}(A \times B)$, then $p\left(W^{1}\right)+p\left(W^{2}\right)=1$. In that case, $v_{1}^{p}=v_{1}^{o}$ and $v_{2}^{\mathrm{p}}=v_{2}^{\mathrm{o}}$.

[3] When player 1 is optimistic and player 2 is pessimistic, the resulting game is zero-sum, just like the original game (in pure strategies). Naturally, this also holds when player 1 is pessimistic and player 2 is optimistic. However, when both players are optimistic or both are pessimistic, the game in mixed strategies need not be zero-sum anymore.

optimal strategies A strategy $p_{1} \in \Delta(A)$ is optimistic optimal for player 1 if

$$
<\mathrm{p}_{1}, \mathrm{p}_{2}>\left(\mathrm{W}^{1}\right) \geqslant v_{1}^{\mathrm{o}}
$$

for all $p_{2} \in \Delta(B)$. Similarly, $p_{1}$ is pessimistic optimal for player 1 if

$$
1-<\mathrm{p}_{1}, \mathrm{p}_{2}>\left(\mathrm{W}^{2}\right) \geqslant v_{1}^{\mathrm{p}}
$$

for all $p_{2} \in \Delta(B)$. For player 2, a strategy $p_{2} \in \Delta(B)$ is pessimistic optimal if for all strategies $p_{1} \in \Delta(A)$ it holds that $\left\langle p_{1}, p_{2}>\left(W^{1}\right) \leqslant v_{2}^{p}\right.$. Strategy $p_{2}$ is optimistic optimal for player 2 if for all strategies $p_{1} \in \Delta(A)$ it holds that $1-<\mathrm{p}_{1}, \mathrm{p}_{2}>\left(\mathrm{W}^{2}\right) \leqslant v_{2}^{\mathrm{o}}$.

\subsection{THE MAIN RESULTS}

In this section we present our main results. The proofs of the main results, Theorems 4.4.1, 4.4.2, and 4.4.6, can be found in Appendix 4.A. As a reminder we note that throughout this section we assume that $\mathcal{F}(A)$ satisfies condition $[A]$, and $\mathcal{F}(B)$ satisfies condition $[B]$. The first result states that the guarantee levels for player 2 are always equal to 1 .

Theorem 4.4.1. The guarantee levels for player 2 are $\nu_{2}^{p}=v_{2}^{o}=1$. In both cases (optimistic and pessimistic), any strategy of player 2 is optimal.

The analysis of the guarantee levels for player 1 requires more effort. We distinguish three cases, in which the levels for player 1 may differ. It is peculiar that the pessimistic and optimistic approach can make a difference only in one case. 
Theorem 4.4.2. For player 1, we distinguish three exhaustive but mutually exclusive settings.

[1] Suppose there exists no non-trivial measure on $\mathcal{F}(A)$. Then $v_{1}^{p}=v_{1}^{o}=0$. In both cases (optimistic and pessimistic), any strategy of player 1 is optimal.

[2] Suppose there exists an atomless measure on $\mathcal{F}(A)$. Assume condition [C]. Then $v_{1}^{\mathrm{p}}=v_{1}^{\mathrm{o}}=1$. In both cases (optimistic and pessimistic), any atomless probability measure is optimal for player 1 .

[3] Suppose there is a non-trivial measure on $\mathcal{F}(A)$, but each non-trivial measure has an atom. Then $v_{1}^{o}=1$. Moreover, any non-trivial $\{0,1\}$-valued probability measure on $\mathrm{A}$ is optimistic optimal for player 1.

The following table summarizes the results for player 1's guarantee levels in Theorem 4.4.2:

\begin{tabular}{|l|l|c|c|}
\hline & & $v_{1}^{\mathrm{p}}$ & $v_{1}^{\mathrm{o}}$ \\
\hline Case [1] & No non-trivial measure on $\mathcal{F}(A)$ & $\mathrm{O}$ & $\mathrm{O}$ \\
\hline Case [2] & Atomless measure on $\mathcal{F}(A)$ & 1 & 1 \\
\hline Case [3] & Non-trivial measure on $\mathcal{F}(A)$, and each has an atom & $?$ & 1 \\
\hline
\end{tabular}

Thus, the guarantee levels of player 1 depend on what type of (probability) measures player 1's action space $A$ admits. For each case, we present an example. In the first two examples we do not specify the sigma-algebra $\mathcal{F}(B)$, we only require that the conditions from Section 4.3 are satisfied. In Example 3 we need an additional requirement on $\mathcal{F}(B)$.

Example 4.4.3. Take $A=\mathbb{N}$ and $\mathcal{F}(A)=2^{\mathbb{N}}$. Since $A=\mathbb{N}$ is countable, $\mathcal{F}(A)$ does not admit a non-trivial measure. So, this example belongs to case [1]. Hence, $v_{1}^{\mathrm{p}}=v_{1}^{\mathrm{o}}=0$.

Example 4.4.4. Take $A=[0,1]$. Let $\mathcal{F}(A)=\mathcal{M}$ be the collection of Lebesgue measurable subsets of $[0,1]$. Then, the Lebesgue measure is an atomless measure on $\mathcal{M}$. So, this example belongs to case [2]. Hence, $v_{1}^{\mathfrak{p}}=v_{1}^{\mathrm{o}}=1$. $\diamond \diamond$

Example 4.4.5. Take $A=[0,1]$. Let $\mathcal{F}(A)$ consist of all subsets of $A=[0,1]$ that are at most countable, together with their complements. In Lemma 4.A.I it is shown that this example falls under case [3]. Hence, $v_{1}^{\mathrm{o}}=1$, and the 
unique non-trivial probability measure on $\mathcal{F}(A)$ is the only optimistic optimal strategy for player 1 .

Remark. The value of a game exists when the guarantee levels for the two players coincide. In finite games the value always exists, but for infinite games this is not necessarily so. Since in catch games the guarantee level for player 2 is always 1, the value exists when player 1's guarantee level is also 1. So the value exists in a catch game in case [2], and in case [3] when player 1 is optimistic.

In general, the guarantee level of $v_{1}^{p}$ for a pessimistic player 1 remains an open problem in case [3]. We briefly discuss a partial result. We assume that $A$ is uncountable. ${ }^{4}$ The sigma-algebra $\mathcal{F}(A)$ on $A$ that consists of all subsets of $A$ that are at most countable, together with their complements is called the minimal sigma-algebra on $A$. Note that the minimal sigma-algebra on $A$ satisfies condition [A].

For the pessimistic case, we have the following partial result.

Theorem 4.4.6. Let $\mathrm{A}$ be uncountable. Let $\mathcal{F}(\mathrm{A})$ be the minimal sigma-algebra on A. Let $\mathcal{F}(\mathrm{B})$ be the smallest sigma-algebra that satisfies condition [B]. Then $v_{1}^{p}=0$. Moreover, any strategy is pessimistic optimal for player 1.

We conclude that in case [3] the optimistic and pessimistic guarantee levels for player 1 may differ. We conjecture that $v_{1}^{p}=0$ is valid in general in case [3].

\subsection{CONSEQUENCES FROM SET THEORY}

In this section we discuss the consequences of set theory on player 1's guarantee levels in our main theorem. In Theorem 4.4.2 we distinguished three exhaustive but mutually exclusive cases depending on what type of (probability) measures player 1 's action set $A$ admits. Our examples showed that different choices for $\mathcal{F}(A)$ may change the case we end up in.

In this section we fix the choice of sigma-algebra for player 1 to $\mathcal{F}(A)=2^{A}$. Thus, the case distinctions now boil down to the question of existence of

4 Note that for countable sets A we are automatically in case [1]. Moreover, then the guarantee levels are the classical guarantee levels from Section 3.4.4. 
certain types of probability measures defined on the entire power set of A. In this section we provide more details. For each case, we present well-known axioms from set theory that force our model to be in that specific case. The definitions of the various axioms can be found in Appendix 4.C. In subsection 4.5.4 we provide an example to illustrate our results in this section.

\subsubsection{Case $[A]$}

In case [A] we assume the Zermelo-Fraenkel axioms ZF, plus the axiom of constructability $\mathrm{V}=\mathrm{L}$. We show that under these conditions, $2^{\mathrm{A}}$ belongs to case [1] for any set $A$. It can be shown that, if ZF is consistent, then ZF plus " $\mathrm{V}=\mathrm{L}$ " is consistent.

Theorem 4.5.1. Assume the axiom of constructability V $=\mathrm{L}$. Then there exists no non-trivial measure on $2^{\mathrm{A}}$. Hence

$$
v_{1}^{\mathrm{p}}=v_{1}^{\mathrm{o}}=0 .
$$

Proof. Let $A$ be any infinite set. We show that there exists no non-trivial measure on $2^{A}$. Suppose the opposite. Take the smallest cardinal $\kappa$ on which a non-trivial measure $\mu$ exists. Thus, $k \leqslant \operatorname{card}(A)$.

Suppose first that $\kappa>2^{\mathrm{N}_{0}}$. By corollary 10.7 in Jech (2006), $\mathrm{k}$ is a real-valued measurable cardinal. Hence, by corollary 10.10 in Jech (2006), $k$ is simply a measurable cardinal. This is however impossible in view of Scott's theorem (Jech (2006), Theorem 17.1), which states that under the assumption of the axiom of constructability $\mathrm{V}=\mathrm{L}$, there is no measurable cardinal.

So, $k \leqslant 2^{x_{0}}$. Then, the measure $\mu$ can be extended to a non-trivial measure $\bar{\mu}$ on $2^{\aleph_{0}}$. This is however impossible. Based on Gödel's theorem (Jech (2006), Theorem 13.20) under the assumption of the axiom of constructability $V=L$, the generalized continuum hypothesis holds: $2^{\aleph_{\alpha}}=\aleph_{\alpha+1}$ for every ordinal $\alpha$. Banach and Kuratowski showed that under the assumption of the continuum hypothesis $2^{\aleph_{0}}=\aleph_{1}$, there exists no non-trivial measure on the continuum $2^{\aleph_{0}}$, i.e. on any set having the same cardinality as $[0,1]$ (Jech (2006), pp. 133 and 138 ).

We conclude that there exists no non-trivial measure on $2^{\mathrm{A}}$. Thus, we are in case [1] of Theorem 4.4.2. Hence, $v_{1}^{p}=v_{1}^{o}=0$. 
Remark. We briefly mention that $\mathcal{F}(A)=2^{A}$ falls under case [ 1 ] when $A$ is countable, and when $A$ is a subset of $\mathbb{R}$ or $\mathbb{R}^{n}$, provided that the continuum hypothesis $\mathrm{CH}$ is valid.

\subsubsection{Case [B]}

In case [B] we assume the Zermelo-Fraenkel axioms ZF plus the axiom of determinacy $\mathrm{AD}$. We show that, under these conditions $2^{A}$ belongs to case [2] for any set $A$ whose cardinality exceeds, or equals, the cardinality of $\mathbb{R}$. ${ }^{5}$ Woodin showed that the consistency of $\mathrm{ZF}+\mathrm{AD}$ is equivalent to the consistency of ZF plus the statement that infinitely many Woodin cardinals exist.

Theorem 4.5.2. Assume the axiom of determinacy AD. Let A be such that the cardinality of $\mathrm{A}$ is at least the cardinality of $[0,1]$. Then there exists an atomless measure on $2^{\mathrm{A}}$. Hence

$$
v_{1}^{\mathrm{p}}=v_{1}^{\mathrm{o}}=1 .
$$

Proof. Let $A$ be any infinite set with a cardinality at least the cardinality $[0,1]$. By the axiom of determinacy, $2^{[0,1]}=\mathcal{M}$. So, the Lebesgue measure is an atomless probability measure on $2^{[0,1]}$. Since the cardinality of $A$ is at least the cardinality of $[0,1]$, we can extend this measure to a measure on $2^{\mathrm{A}}$.

Remark. It is known that the existence of an atomless measure (on any $2^{A}$ ) is equivalent to the existence of a countably additive extension of the Lebesgue measure to all sets of the real numbers.

\subsubsection{Case $[C]$}

In case $[\mathrm{C}]$ we assume ZFC (ZF plus AC), existence of a measurable cardinal $\mathrm{MC}$, and the continuum hypothesis $\mathrm{CH}$. In this case we show that $2^{A}$ belongs to case [3], whenever the cardinality of $A$ is larger or equal to the cardinality of a measurable cardinal. In ZF plus MC it can be shown that ZF is consistent. It was shown by Lévy and Solovay (1967) that if ZFM (ZF plus MC) is consistent, then $\mathrm{ZFM}$ plus $\mathrm{CH}$ is consistent.

5 The cardinality of $X$ is at least the cardinality of $Y$ if there exists a one-to-one map from $Y$ to $\mathrm{X}$. 
Theorem 4.5.3. Assume ZFC and $\mathrm{CH}$. Also assume MC, so there exists a measurable cardinal $\alpha$. Let $A$ be such that $\operatorname{card}(A) \geqslant \alpha$. Then there exists a non-trivial measure on $2^{A}$, and each such measure has an atom. Hence

$$
v_{1}^{\mathrm{o}}=1
$$

Proof. We show that $2^{A}$ belongs to case [3]. Since $\alpha$ is a measurable cardinal, and $\operatorname{card}(A) \geqslant \alpha$, it follows that there is a non-trivial probability measure on $2^{A}$. Let $\mu$ be such a non-trivial probability measure on $2^{A}$. We show that $\mu$ has an atom.

Suppose that $\mu$ does not have an atom. Lemma 10.9 of Jech (2006) states that, if there is an atomless non-trivial $\sigma$-additive measure on $A$, then there exists a non-trivial countably additive measure on some cardinal $k \leqslant 2^{\aleph_{0}}$. So, there exists a non-trivial measure on some $\mathrm{k} \leqslant 2^{\mathrm{x}_{0}}$. Since by $\mathrm{CH}$ we have that $2^{\aleph_{0}}=\aleph_{1}=\omega_{1}$, this implies that there exists a non-trivial measure on $\omega_{1}$. This however contradicts Lemma 10.13 in Jech (2006). This concludes the proof.

\subsubsection{An example}

This section can be considered to be a simpler, illustrative version of the preceding discussion. We explain how the various set theoretic settings affect the analysis for the specific case $A=[0,1]$ and $\mathcal{F}(A)=2^{A}$.

Example 4.5.4. Take $A=[0,1]$ and $\mathcal{F}(A)=2^{A}=2^{[0,1]}$.

[A] Assume ZF and the axiom of constructibility $\mathrm{V}=\mathrm{L}$. Then, based on Theorem 4.5.1, $2^{\mathrm{A}}$ belongs to case [1]. So, $v_{1}^{\mathrm{p}}=v_{1}^{\mathrm{o}}=0$. Note that, in this setting, also $\mathrm{AC}$ is valid, so that $2^{A} \neq \mathcal{M}$.

[B] Assume ZF and AD. Then, it follows from Mycielski-Świerczkowski (1964) that $2^{[0,1]}=\mathcal{M}$. So, we are in fact in the situation of Example 2. It follows that $v_{1}^{\mathrm{p}}=v_{1}^{\mathrm{o}}=1$.

[C] Assume ZFC and $\mathrm{CH}$. Suppose that there exists a non-trivial measure on $2^{[0,1]}$. Then there exists a non-trivial measure on $2^{\aleph_{0}}$. Banach and Kuratowski showed that this implies that $2^{\aleph_{0}}>\aleph_{1}$. This violates $\mathrm{CH}$. 
Hence, under these assumptions, $2^{\mathrm{A}}$ does not belong to case [3], but to case [1].

This ends our discussion of the example.

\subsection{Finitely additive strategies}

In this section we will compare the setup with countable additivity with that of finite additivity. For more on finite additivity, see the Related literature sections of Chapters 1 and 3 .

We show that the guarantee levels defined using finitely additive strategies differ from those specified in Theorems 4.4.1 and 4.4.2, where the player used countably additive strategies. We restrict our analysis to the case where the sigma-algebra equals the power set of the action space.

Let $S$ be a non-empty set. A probability charge on $S$ is a function $\mu: 2^{S} \rightarrow[0,1]$ such that $\mu(S)=1$, and for all disjoint sets $E, F \in 2^{S}$ it holds that $\mu(E \cup F)=$ $\mu(E)+\mu(F)$. We denote by $\mathcal{C}(S)$ the set of all probability charges on $2^{S}$. If $S$ is infinite and we assume the Axiom of Choice, the set of (countably additive) probability measures $\Delta(S)$ is a strict subset of $\mathcal{C}(S)$. For more on probability charges, see Chapter 2.

We can define an outer measure for probability charges very similarly to how it was carried out in the Preliminaries. However, in this case we would use only finite covers instead of countable ones. Furthermore, the guarantee levels can be defined similarly to Section $4 \cdot 3$, but now the set of strategies for player 1 is $\mathcal{C}(A)$, and the set of strategies for player 2 is $\mathcal{C}(B)$. We denote the 4 guarantee levels defined through probability charges by $w_{1}^{\mathrm{p}}, w_{1}^{\mathrm{o}}, w_{2}^{\mathrm{p}}$ and $w_{2}^{\mathrm{o}}$, which are special cases of Definition 3.4.2. The following result follows from Theorems 3.4.3 and 3.5.2.

Theorem 4.6.1. Assume the Axiom of Choice. The guarantee levels in finitely additive strategies are

$$
w_{1}^{\mathrm{p}}=w_{2}^{\mathrm{o}}=0 \text { and } w_{1}^{\mathrm{o}}=w_{2}^{\mathrm{p}}=1 .
$$

Both players have pessimistic and optimistic optimal strategies.

So optimal strategies always exist, independent of assuming finitely or countably additive strategies. 
However, the guarantee levels differ drastically between the two different models. The finitely additive guarantee levels are 0 when the definition of a guarantee level considers 1 minus the outer-measure of $W^{2}$ (equations (3) and (5)), and the guarantee levels are 1 when the definition considers the outer-measure of $W^{1}$ (equations (2) and (4)). Thus the finitely additive guarantee levels of a player depend on whether we take the pessimistic or the optimistic approach.

Also note that, when the players agree on how to compute expected payoffsthat is, when one player is pessimistic and the other player is optimistic-in the finitely additive case, the players agree on the value of the game. This in stark contrast to for example, case [1] of the countably additive setup, where both players think that their opponent will win, even when they agree on how to compute expected payoffs.

\subsection{REMARKS ON OUR REQUIREMENTS FOR THE SIGMA-ALGEBRAS}

In this section, we briefly examine how demanding conditions $[A],[B]$, and $[\mathrm{C}]$ are. We argue that conditions $[\mathrm{A}]$ and $[\mathrm{B}]$ are relatively mild, while condition $[\mathrm{C}]$ is more demanding.

We start with condition $[A]$ on $\mathscr{F}(A)$. The condition requires that all singleton sets are in $\mathcal{F}(A)$. This is a relatively mild condition. It is for example satisfied by any sigma-algebra that includes a Hausdorff topology. Thus, any Borel sigma-algebra induced by such a topology, as well as the familiar sigmaalgebra $\mathcal{M}$ on $\mathbb{R}$ satisfy this condition. One of the consequences of this condition - from a game theoretic perspective - is that it allows for pure strategies. In order to argue that condition $[\mathrm{B}]$ on $\mathcal{F}(\mathrm{B})$ is mild, we need a bit more terminology.

A subset $L$ of $B$ is called a layer if there is a finite subset $F$ of $A$ such that $\mathrm{L}=\{\mathrm{F} \cup\{\mathrm{a}\}: \mathrm{a} \in \mathrm{A} \backslash \mathrm{F}\}$.

Remarks. Note that every finite set $\mathrm{F}$ defines a (unique) layer. Moreover, if $L$ is a layer generated by $F$, then $L \subseteq B_{m}$ with $m=|F|+1$. Also note that $B_{1}$ is a layer (for $F=\emptyset$ ), and that condition $[B]$ implies that every layer is an element of $\mathcal{F}(\mathrm{B})$. 
A set $C \subseteq B$ is minimalistic if for every layer $L$, either $L \cap C$ is at most countable, or $\mathrm{L} \backslash \mathrm{C}$ is at most countable.

Condition $[\mathrm{M}]$ For every $\mathrm{C} \in \mathcal{F}(\mathrm{B}), \mathrm{C}$ is minimalistic.

We have the following result. The proof can be found in Appendix 4.A.

Lemma 4.7.1. Let $\mathcal{F}(\mathrm{B})$ be the smallest sigma-algebra that satisfies condition [B]. Then $\mathcal{F}(\mathrm{B})$ satisfies condition [M].

Thus, no element of the smallest sigma-algebra that satisfies condition [B] will split a layer into two uncountable sets. In this sense, $\mathcal{F}(B)$ only contains a limited collection of subsets of B.

Remark. Suppose that $\mathcal{F}(A)$ is not minimal. Then any sigma-algebra $\mathcal{F}(B)$ that satisfies condition [C] automatically violates condition [M]. To see this, since $\mathcal{F}(A)$ is not minimal, $\mathcal{F}(A)$ contains an uncountable set $D$ whose complement is also uncountable. ${ }^{6}$ Then $Y(D, 1) \in \mathcal{F}(B)$ by $[C]$. However, $L=B_{1}$ is a layer-for $F=\emptyset$ - while both $Y(D, 1) \cap L$ and $L \backslash Y(D, 1)$ are uncountable. Hence, $Y(D, 1)$ is not minimalistic.

Hence, when $\mathcal{F}(A)$ is not minimal, the smallest sigma-algebra that satisfies condition [B] does not satisfy condition [C]. The smallest sigma-algebra that satisfies condition [B] is a strict subset of any sigma-algebra that satisfies condition $[C]$. Nevertheless, given $\mathcal{F}(A)$, also condition $[C]$ still leaves some flexibility for the choice of $\mathcal{F}(B)$. For a set $D \subseteq A$, write

$$
D^{*}=\{\{a\}: a \in D\} .
$$

Let $\mathcal{F}(B)$ be the smallest sigma-algebra that satisfies condition $[\mathrm{C}]$. Then

$$
\left\{\mathrm{E} \subseteq \mathrm{B}_{1}: \mathrm{E} \in \mathcal{F}(\mathrm{B})\right\}=\left\{\mathrm{D}^{*}: \mathrm{D} \in \mathcal{F}(\mathrm{A})\right\}
$$

Thus, the restriction of $\mathcal{F}(B)$ to $B_{1}$ is "identical" to $\mathcal{F}(A)$.

\subsection{CONCLUDING REMARKS}

We examined a class of zero-sum games, catch games, in which the guarantee levels are quite varied. The countably additive levels depend on many details

6 This statement is not entirely innocuous. Showing the existence of such a set D for any given uncountable set $A$ requires Lemma of Zorn, hence AC. In our case it follows though from the assumption that $\mathcal{F}(A)$ is not minimal. 
of the model, including the cardinality of the action set, the sigma-algebra defined on the action set, and on set theoretic axioms.

At first sight, when $A$ is infinite, as player 2 can only choose finite subsets of $A$, it would seem that player 2 has an impossible task. We showed that this speculation is just partly justified, and the situation is more complex than first intuition might suggest.

We distinguished three cases. In case [1] player 1 only has trivial strategies. This makes it easier to catch him, which is reflected in his guarantee levels being 0 , signifying that player 1 expects to be caught. Paradoxically, player 2 disagrees, and thinks player 1 will win the game.

In case [2] player 1 has atomless measures, which is more favorable when trying not to be caught. The guarantee levels for player 1 are 1 in this case. So, both players expect player 1 to win the game.

Case 3 is an intermediate case. The optimistic guarantee level for player 1 is again 1 . We also established that $v_{1}^{p}=0$ for a specific type of sigma-algebra. In general, it remains an open problem to determine $v_{1}^{\mathrm{p}}$ for this case.

The situation changes again when we consider finitely additive strategies. In this case the guarantee levels of the players are driven by the extension (pessimistic or optimistic) we take. When player 1 is optimistic, and player 2 is pessimistic, the value of the game is 1 . So, both players expect player 1 to win the game. When player 1 is pessimistic, and player 2 is optimistic, both players expect player 2 to win the game.

Thus, we should be cautious with claims about the value of a zero-sum game. As we argued, the value, its size, and even its existence, may depend on many details in our choices regarding which model to select.

In economic theory there is an abundance of models with infinite action spaces. Modeling the mixed strategies of players in this case is not trivial, since similar questions can be raised to the ones presented in this chapter. In our examples the exact model of mixed strategies significantly changed the prediction, both in terms of the value of the game and in terms of optimal strategies. 
In the previous chapter we considered bounded payoff functions, and we focused on different ways of defining finitely additive values and comparing them to each other. We only briefly discussed countably additive guarantee levels, only in Section 3.4.4. In this chapter we considered a subset of zerosum games, and we only considered the pessimistic and optimistic guarantee levels from Chapter 3. We focused on the countably additive guarantee levels and varied them in different ways.

In this chapter and the previous one we considered two-player simultaneousmove games where different models of probabilities can have an effect on the value of the game, because players have infinite action spaces. In the next chapter we consider one-player games-decision problems-with infinitely many time periods, where interesting cases can arise already with finitely many actions.

\section{A THE PROOFS OF THEOREMS 4.4.1 AND $4 \cdot 4.2$}

In this section we provide the proofs of our main theorems. Throughout this section, we assume that $\mathcal{F}(A)$ satisfies condition $[A]$. So, $\{a\} \in \mathcal{F}(A)$ for every $a \in A$. Also, unless explicitly stated otherwise, $\mathcal{F}(B)$ satisfies condition [B]. Thus, $\mathrm{Q}(\mathrm{I}, \mathrm{m}) \in \mathcal{F}(\mathrm{B})$ for any choice of $\mathrm{I}$ and $\mathrm{m}$.

A. The guarantee levels for player 2 We prove the following theorem.

Theorem. The guarantee levels for player 2 are $v_{2}^{p}=v_{2}^{o}=1$. In both cases (optimistic and pessimistic), any strategy of player 2 is optimal.

Proof. We show that

$$
v_{2}^{p}=v_{2}^{o}=1 .
$$

Since $v_{2}^{\mathrm{o}} \leqslant v_{2}^{\mathrm{p}}$, it is sufficient to prove that $v_{2}^{\mathrm{o}}=1$. Take an arbitrary $k \in \mathbb{N}$. We prove that $v_{2}^{o} \geqslant 1-\frac{1}{k}$. For this purpose, take a $p_{2} \in \Delta(\mathrm{B})$. We construct a $p_{1} \in \Delta(A)$ such that

$$
1-<p_{1}, p_{2}>\left(W^{2}\right) \geqslant 1-\frac{1}{k}
$$

For $m \in \mathbb{N}$, write $C_{m}=B_{1} \cup \cdots \cup B_{m}$. By condition [BI], $C_{m} \in \mathcal{F}(B)$ for each $m$. Choose $m \in \mathbb{N}$ such that

$$
\mathrm{p}_{2}\left(\mathrm{C}_{\mathrm{m}}\right) \geqslant 1-\frac{1}{2 \mathrm{k}}
$$


Since $A$ is infinite, we can choose a sequence $a_{1}, \ldots, a_{2 k m}$ of $2 \mathrm{~km}$ different elements of $A$. Since by condition $[A]$ singleton sets are elements of $\mathcal{F}(A)$, we can define

$$
p_{1}=\frac{1}{2 k m} \cdot \delta_{a_{1}}+\ldots+\frac{1}{2 k m} \cdot \delta_{a_{2 k m}},
$$

where $\delta_{a}$ denotes the Dirac measure on $A$. We show that $\left\langle p_{1}, p_{2}>\left(W^{2}\right) \leqslant\right.$ $\frac{1}{\mathrm{k}}$.

Let $A^{*}=\left\{a_{1}, \ldots, a_{2 k m}\right\}$. For each $I \subseteq A^{*}$ with $|I| \leqslant m$, we define $R(I)$ in $A \times C_{m}$ as follows. Define $R(I)_{1}=I \cup\left(A \backslash A^{*}\right)$, and $R(I)_{2}=\left\{b \in C_{m}\right.$ : $\left.\mathrm{b} \cap A^{*}=\mathrm{I}\right\}$. Define

$$
\mathrm{R}(\mathrm{I})=\mathrm{R}(\mathrm{I})_{1} \times \mathrm{R}(\mathrm{I})_{2} .
$$

By condition $[B]$, since

$$
\mathrm{R}(\mathrm{I})_{2}=\mathrm{C}_{\mathrm{m}} \bigcap\left[\left(\bigcup_{a \in \mathrm{I}} \mathrm{B}(\mathrm{a})\right) \backslash\left(\bigcup_{a \in A^{*} \backslash \mathrm{I}} \mathrm{B}(\mathrm{a})\right)\right],
$$

we have that $R(I)_{2} \in \mathcal{F}(B)$. Also, since singleton sets are in $\mathcal{F}(A)$, it follows that $R(I)_{1} \in \mathcal{F}(A)$. So, $R(I)$ is a rectangle. Let $\mathcal{R}$ denote the set of all such sets $R(I)$ for each choice of I. Clearly, $\mathcal{R}$ is finite. We prove the following claim.

Claim. The collection $\mathcal{R} \cup\left\{A \times\left(B \backslash C_{m}\right)\right\}$ is a cover of $W^{2}$.

Proof of claim. Take an $(a, b) \in W^{2} \cap\left(A \times C_{m}\right)$. Then $a \in b$. Take $I=b \cap A^{*}$. Note that, since $b \in C_{m}$, the set I has at most $m$ elements. Consider the rectangle $R(I) \in \mathcal{R}$. We argue that $(a, b) \in R(I)$. Clearly,

$$
\mathrm{b} \in\left\{\mathrm{b} \in \mathrm{C}_{\mathrm{m}}: \mathrm{b} \cap \mathrm{A}^{*}=\mathrm{I}\right\}
$$

by definition of I. We argue that $a \in I \cup\left(A \backslash A^{*}\right)$. Suppose that $a \notin I$. Then, since $a \in b, a \notin A^{*}$. So, $a \in A \backslash A^{*}$. End proof of claim.

We continue to prove that $\left\langle p_{1}, p_{2}>\left(W^{2}\right) \leqslant \frac{1}{k}\right.$. By the choice of $m$, we have

$$
<p_{1}, p_{2}>\left(A \times\left(B \backslash C_{m}\right)\right)=p_{1}(A) \cdot p_{2}\left(B \backslash C_{m}\right) \leqslant \frac{1}{2 k} .
$$

Next, take $R(I) \in \mathcal{R}$. Define $k(I)=p_{2}\left(R(I)_{2}\right)$. Then

$$
\begin{aligned}
<\mathrm{p}_{1}, \mathrm{p}_{2}>(\mathrm{R}(\mathrm{I})) & =\mathrm{p}_{1}\left(\mathrm{R}(\mathrm{I})_{1}\right) \cdot \mathrm{p}_{2}\left(\mathrm{R}(\mathrm{I})_{2}\right)=\mathrm{p}_{1}(\mathrm{I}) \cdot \mathrm{k}(\mathrm{I}) \\
& \leqslant \mathrm{m} \cdot \frac{1}{2 \mathrm{~km}} \cdot \mathrm{k}(\mathrm{I})=\frac{1}{2 \mathrm{k}} \cdot \mathrm{k}(\mathrm{I}) .
\end{aligned}
$$


Also, let J be the collection of subsets I of $A^{*}$ with $|I| \leqslant m$. Then the collection $\left\{R(I)_{2}\right\}_{I \in \mathcal{J}}$ constitutes a finite partition of $C_{m}$. Indeed, $b \in R(I)_{2}$ precisely when $\mathrm{I}=\mathrm{b} \cap \mathrm{A}^{*}$. Hence, using the above claim, and the fact that

$$
\sum_{\mathrm{I} \in \mathcal{J}} k(\mathrm{I})=\mathrm{p}_{2}\left(\mathrm{C}_{\mathrm{m}}\right) \leqslant 1,
$$

we have that

$$
\begin{aligned}
<\mathrm{p}_{1}, \mathrm{p}_{2}>\left(\mathrm{W}^{2}\right) & \leqslant<\mathrm{p}_{1}, \mathrm{p}_{2}>\left(\mathrm{A} \times\left(\mathrm{B} \backslash \mathrm{C}_{\mathrm{m}}\right)\right)+\sum_{\mathrm{I} \in \mathcal{J}}<\mathrm{p}_{1}, \mathrm{p}_{2}>(\mathrm{R}(\mathrm{I})) \\
& \leqslant \frac{1}{2 \mathrm{k}}+\sum_{\mathrm{I} \in \mathcal{J}} \frac{1}{2 \mathrm{k}} \cdot \mathrm{k}(\mathrm{I}) \\
& \leqslant \frac{1}{2 \mathrm{k}}+\frac{1}{2 \mathrm{k}}=\frac{1}{\mathrm{k}}
\end{aligned}
$$

Since the guarantee levels for player 2 are 1 , any probability measure $p_{2} \in$ $\Delta(\mathrm{B})$ is automatically pessimistic and optimistic optimal for player 2 .

B. The guarantee levels for player $\mathbf{I}$ in case [1] In this section, we assume that there exists no non-trivial measure on $\mathcal{F}(A)$. We also only need condition [B3] on $\mathcal{F}(B)$ instead of [B].

Theorem. Assume that there exists no non-trivial measure on $\mathcal{F}(A)$. Then

$$
v_{1}^{p}=v_{1}^{o}=0
$$

In both cases (optimistic and pessimistic), any strategy of player 1 is optimal.

Proof. Since there exists no non-trivial measure on $\mathcal{F}(A)$, by Lemma 4.B.1, every strategy of player 1 has a countable support. Take a $p_{1} \in \Delta(A)$ and let $C$ be the countable support for $p_{1}$. Let $\varepsilon>0$. Take a finite subset $D \subseteq C$ such that $p_{1}(D) \geqslant 1-\varepsilon$. By condition [B3], $\{D\} \in \mathcal{F}(B)$. So, $\delta_{D} \in \Delta(B)$, and $<p_{1}, \delta_{D}>\left(W^{1}\right) \leqslant \varepsilon$. Hence

$$
\inf _{p_{2} \in \Delta(B)}<p_{1}, p_{2}>\left(W^{1}\right)=0
$$

Therefore $v_{1}^{\mathrm{o}}=0$, which implies that $v_{1}^{\mathrm{p}}=0$ as well. Since these levels for player 1 are 0 , it follows that all probability measures $p_{1} \in \Delta(A)$ are pessimistic and optimistic optimal for player 1 .

C. The guarantee levels for player 1 in case [2] In this section, assume condition $[C]$. We also assume that there is an atomless measure on $\mathcal{F}(A)$. 
Theorem. Assume condition [C]. Further assume that there is an atomless measure on $\mathcal{F}(A)$. Then

$$
v_{1}^{\mathrm{p}}=v_{1}^{\mathrm{o}}=1 .
$$

In both the optimistic and the pessimistic case, any atomless probability measure is optimal for player 1 .

Proof. Let $p_{1}$ be any atomless probability measure on $A$. It is sufficient to prove that $v_{1}^{p}=1$. Thus, it is sufficient to prove that $\left\langle\mathrm{p}_{1}, \mathrm{p}_{2}\right\rangle\left(\mathrm{W}^{2}\right)=0$ for every $p_{2} \in \Delta(B)$. Take any $p_{2} \in \Delta(B)$ and $k \in \mathbb{N}$. We prove that

$$
<\mathrm{p}_{1}, \mathrm{p}_{2}>\left(\mathrm{W}^{2}\right) \leqslant \frac{1}{\mathrm{k}} \text {. }
$$

For every $m \in \mathbb{N}$, by Theorem 4.B.2 there exists a partition $\mathcal{A}=\left\{A_{\mathrm{m}}^{1}, \ldots, A_{\mathrm{m}}^{\mathrm{km}}\right\}$ of $A$ such that $p_{1}\left(A_{m}^{i}\right)=\frac{1}{k m}$ for each $i=1, \ldots, k m$.

For a vector $g=(g(1), \ldots, g(k m))$ whose coordinates are non-negative integers, write

$$
X(g, m)=\bigcup_{\ell: g(\ell)>0} A_{m}^{\ell}
$$

$Y(g, m)=\left\{b \in B_{m}: b \cap A_{m}^{\ell}\right.$ has $g(\ell)$ elements for every $\left.\ell=1, \ldots, k m\right\}$, and

$$
Z(g, m)=X(g, m) \times Y(g, m) .
$$

For fixed $m$, let $Z(m)$ denote the collection of all such sets $Z(g, m)$, and let $z$ denote the collection of all sets $Z(\mathrm{~g}, \mathrm{~m})$. Clearly, $z(\mathrm{~m})$ is finite, and $z$ is countable. We note the following facts.

[1] $Z$ is a cover of $W^{2}$. We first show that each $Z(g, m)$ is a rectangle. Since $X(g, m)$ is a union of sets $A_{m}^{\ell}$, it follows that $X(g, m) \in \mathcal{F}(A)$. Also, since $Y(g, m)$ is the intersection of $B_{m}$ with sets of the form $Y\left(A_{m}^{\ell}, g(\ell)\right)$, it follows from condition $[\mathrm{C}]$ that $Y(\mathrm{~g}, \mathrm{~m}) \in \mathcal{F}(\mathrm{B})$.

Next, take any $(a, b) \in W^{2}$. Define $m=|b|$. Define $g$ by $g(\ell)=\left|b \cap A_{m}^{\ell}\right|$. Then $b \in Y(g, m)$. Further, take $\ell$ with $a \in A_{m}^{\ell}$. Since $(a, b) \in W^{2}$, we know that $a \in b$. So $g(\ell)>0$. Thus, $a \in X(g, m)$. It follows that $(a, b) \in Z(g, m)$. Hence, the sets $Z(g, m)$ cover $W^{2}$.

[2] For any non-empty set $Z(g, m) \in Z$ we have

$$
p_{1}(X(g, m))=\sum_{\ell: g(\ell)>0} p_{1}\left(A_{m}^{\ell}\right) \leqslant m \cdot \frac{1}{k m}=\frac{1}{k} .
$$


[3] For fixed $m$, the sets $Y(g, m)$ constitute a partition of $B_{m}$. To see this, note that for $b \in B_{m}, b \in Y(g, m)$ precisely when each $g(\ell)$ is the number of elements of $b \cap A_{m}^{\ell}$.

Using the properties above, we compute that

$$
\begin{aligned}
<p_{1}, p_{2}>\left(W^{2}\right) & \leqslant \sum_{Z(g, m) \in Z}<p_{1}, p_{2}>(Z(g, m)) \\
& =\sum_{m} \sum_{Z(g, m) \in Z(m)} p_{1}(X(g, m)) \cdot p_{2}(Y(g, m)) \\
& \leqslant \sum_{m} \frac{1}{k} \cdot \sum_{Z(g, m) \in Z(m)} p_{2}(Y(g, m)) \\
& =\sum_{m} \frac{1}{k} \cdot p_{2}\left(B_{m}\right)=\frac{1}{k} .
\end{aligned}
$$

It also follows that $p_{1}$ is pessimistic, and hence optimistic, optimal for player 1 .

D. The guarantee levels for player 1 in case [3] In this section we assume that there is a non-trivial measure on $\mathcal{F}(A)$, but each such measure has an atom.

Theorem. Assume that there is a non-trivial measure on $\mathcal{F}(A)$, but each such measure has an atom. Then

$$
v_{1}^{\mathrm{o}}=1 .
$$

Moreover, any non-trivial $\{0,1\}$-valued measure on $\mathcal{F}(A)$ is optimistic optimal for player 1.

Proof. Let $p_{1}$ be a non-trivial probability measure on $\mathcal{F}(A)$. By our assumption, it has an atom, say $T$. Let $q_{1}$ be the probability measure on $\mathcal{F}(A)$ defined by

$$
q_{1}(E)=\frac{p_{1}(E \cap T)}{p_{1}(T)}
$$

for every $E \in \mathcal{F}(A)$. Note that $q_{1}$ is non-trivial and $\{0,1\}$-valued.

We prove that $v_{1}^{o}=1$. In particular, we show that $\left\langle\mathrm{q}_{1}, \mathrm{p}_{2}>\left(\mathrm{W}^{1}\right)=1\right.$ for every $p_{2} \in \Delta(B)$. Take any $p_{2} \in \Delta(B)$. Let $\left\{Z^{i}\right\}_{i \in \mathbb{N}}$ be a cover of $W^{1}$. We prove that

$$
\sum_{i \in \mathbb{N}}<q_{1}, p_{2}>\left(Z^{i}\right)=1
$$

The proof is in three parts. 
A. Let

$$
E=\left\{i \in \mathbb{N}: q_{1}\left(Z_{1}^{i}\right)=1\right\} \quad \text { and } \quad P=\bigcap_{i \in E} Z_{1}^{i} \cap\left(\bigcap_{i \notin E}\left(A \backslash Z_{1}^{i}\right)\right) .
$$

We argue that $q_{1}(P)=1$. First note that $q_{1}\left(A \backslash Z_{1}^{i}\right)=0$ for all $i \in E$. However, since $q_{1}$ is a $\{0,1\}$-valued measure, we also have $q_{1}\left(Z_{1}^{i}\right)=0$ for all $i \notin E$. It follows that $\mathrm{q}_{1}(A \backslash P)=0$, so that $\mathrm{q}_{1}(\mathrm{P})=1$.

B. Write

$$
\mathrm{Q}=\bigcup_{i \in E} \mathrm{Z}_{2}^{i}
$$

We argue that $\mathrm{Q}=\mathrm{B}$. Suppose there is a fixed $\mathrm{c} \in \mathrm{B}$ with $\mathrm{c} \notin \mathrm{Q}$. We derive a contradiction.

Take any $a \in P$. Take $i \in E$. Then, since $c \notin Q$, also $c \notin Z_{2}^{i}$. It follows that $(a, c) \notin Z^{i}$. Take $i \notin E$. Then, since $a \in P, a \notin Z_{1}^{i}$. So, also in this case, $(a, c) \notin Z^{i}$. We conclude that $(a, c) \notin Z^{i}$ for every $i \in \mathbb{N}$. This implies that $(a, c) \notin W^{1}$. It follows that $a \in c$. So, $P \subseteq c$.

However, as $q_{1}$ is non-trivial and $q_{1}(P)=1$, the set $P$ is uncountable, hence infinite. This contradicts the fact that $c$ is a finite subset of $A$.

C. Therefore, we obtain

$\sum_{i \in \mathbb{N}}<q_{1}, p_{2}>\left(Z^{i}\right)=\sum_{i \in \mathbb{N}} q_{1}\left(Z_{1}^{i}\right) \cdot p_{2}\left(Z_{2}^{i}\right)=\sum_{i \in E} p_{2}\left(Z_{2}^{i}\right) \geqslant p_{2}(Q)=p_{2}(B)=1$.

This completes the proof that $v_{1}^{o}=1$. It also implies that $\mathrm{q}_{1}$ is an optimistic optimal strategy for player 1 .

E. The proofs for the partial result in Theorem 4.4.6. Let $\mathcal{F}(A)$ be the minimal sigma-algebra on $A$. Define $q_{1}$ on $\mathcal{F}(A)$ by, for every $E \in \mathcal{F}(A)$,

$$
q_{1}(E)= \begin{cases}0 & \text { if } E \text { is at most countable } \\ 1 & \text { otherwise }\end{cases}
$$

Notice that $q_{1}$ is a non-trivial probability measure for which each uncountable $E \in \mathcal{F}(A)$ is an atom. We start with the following observation.

Lemma 4.A.1. The probability measure $\mathrm{q}_{1}$ is the unique non-trivial probability measure on $\mathcal{F}(A)$. Hence, $\mathcal{F}(A)$ falls in case [3]. 
Proof. We already observed that $q_{1}$ is a non-trivial measure on $\mathcal{F}(A)$. Let $p_{1}$ be any non-trivial probability measure on $\mathcal{F}(A)$. Then $p_{1}(E)=0=q_{1}(E)$ for every $E \in \mathcal{F}(A)$ that is at most countable. Since $\mathcal{F}(A)$ is minimal, it follows that $\mathrm{p}_{1}=\mathrm{q}_{1}$.

Lemma. Let $\mathcal{F}(\mathrm{B})$ be the smallest sigma-algebra that satisfies condition [B]. Then $\mathcal{F}(\mathrm{B})$ satisfies condition [M].

Proof of Lemma 4.7.1. The proof is in four parts.

A. Take any non-empty set $\mathrm{Q}(\mathrm{I}, \mathrm{m})$. We show that $\mathrm{Q}(\mathrm{I}, \mathrm{m})$ is minimalistic. Take a finite subset $F$. Let $L$ be the layer associated with $F$. Take $b \in L \cap$ $\mathrm{Q}(\mathrm{I}, \mathrm{m})$. Then there is $\mathrm{a} \in \mathrm{A} \backslash \mathrm{F}$ with $\mathrm{b}=\mathrm{F} \cup\{\mathrm{a}\},|\mathrm{F}|=\mathrm{m}-1$, and $\mathrm{I} \subseteq \mathrm{b}$. If $a \in I$. Then $L \cap Q(I, m)=\{b\}$. So, $L \cap Q(I, m)$ is at most countable. If $a \notin I$. Then $\mathrm{I} \subseteq \mathrm{F}$, so that $\mathrm{L} \subseteq \mathrm{Q}(\mathrm{I}, \mathrm{m})$. In that case $\mathrm{L} \backslash \mathrm{Q}(\mathrm{I}, \mathrm{m})$ is empty, hence at most countable.

B. Let $C_{1}, C_{2}, \ldots$ be a sequence of minimalistic subsets of $B$. Write $C=$ $U_{n} C_{n}$. We argue that $C$ is also minimalistic. Let $L$ be any layer. If for each $n$ it holds that $L \cap C_{n}$ is at most countable, then also $L \cap C$ is at most countable. If on the other hand there is an $n$ such that $L \backslash C_{n}$ is at most countable, then clearly also $L \backslash C$ is at most countable.

C. Let $\mathrm{C}$ be a minimalistic subset of $\mathrm{B}$. Then also $\mathrm{B} \backslash \mathrm{C}$ is minimalistic, as for every layer $L, L \cap(B \backslash C)=L \backslash C$ and $L \backslash(B \backslash C)=L \cap C$.

D. Our claim now follows from the observation that the smallest sigmaalgebra that satisfies condition [B] can be constructed by building the countable Borel hierarchy starting with the collection of sets $Q(I, m)$.

Theorem. Let $A$ be uncountable. Let $\mathcal{F}(A)$ be the minimal sigma-algebra on $A$. Let $\mathcal{F}(\mathrm{B})$ be the smallest sigma-algebra that satisfies condition [B]. Then $v_{1}^{\mathrm{p}}=0$. Moreover, any strategy is pessimistic optimal for player 1 .

Proof of Theorem 4.4.6. Let $p_{1} \in \Delta(A)$ be a strategy of player 1 . We have to show that

$$
\begin{aligned}
& \inf _{p_{2} \in \Delta(B)}\left[1-<p_{1}, p_{2}>\left(W^{2}\right)\right]=0, \quad \text { or, equivalently, } \\
& \sup _{p_{2} \in \Delta(B)}<p_{1}, p_{2}>\left(W^{2}\right)=1 .
\end{aligned}
$$


By Lemma 4.B.1 and Lemma 4.A.1 there are a countable set $\mathrm{I} \subseteq \mathrm{A}$ and coefficients $d \geqslant 0$ and $c_{a} \geqslant 0$ for $a \in I$ such that

$$
\mathrm{p}_{1}=\mathrm{d} \cdot \mathrm{q}_{1}+(1-\mathrm{d}) \cdot \sum_{\mathrm{a} \in \mathrm{I}} \mathrm{c}_{\mathrm{a}} \cdot \delta_{\mathrm{a}}
$$

where $q_{1}$ is the unique non-trivial probability measure on $\mathcal{F}(A)$. Take any $\varepsilon>0$. Take a finite set $F \subseteq$ I such that $\sum_{a \notin F} c_{a}<\varepsilon$. If $d=0$, then for $p_{2}=\delta_{F}$ it holds that $<p_{1}, p_{2}>\left(W^{2}\right) \geqslant 1-\varepsilon$.

We assume that $d>0$. Let $L$ be the layer associated with F. For a set $C \in \mathcal{F}(B)$, define

$$
C_{*}=\{a \in A: F \cup\{a\} \in L \cap C\} .
$$

Note that $C_{*}=\{a \in A \backslash F: F \cup\{a\} \in C\}$.

A. We show that $C_{*} \in \mathcal{F}(A)$. Since, by Lemma 4.7.1, $\mathcal{F}(B)$ satisfies condition $[\mathrm{M}]$, either $\mathrm{L} \cap \mathrm{C}$ is at most countable, or $\mathrm{L} \backslash \mathrm{C}$ is at most countable. If $\mathrm{L} \cap \mathrm{C}$ is at most countable, it follows that $C_{*}$ is at most countable. In this case, by condition $[A], C_{*} \in \mathcal{F}(A)$. If $L \backslash C$ is at most countable, the set

$$
\mathrm{D}=\{\mathrm{a} \in \mathrm{A}: \mathrm{F} \cup\{\mathrm{a}\} \in \mathrm{L} \backslash \mathrm{C}\}
$$

is at most countable. So, also in this case $C_{*}=A \backslash(D \cup F)$ is an element of $\mathcal{F}(\mathrm{A})$.

B. Thus, we can define $p_{2}$ by, for every $C \in \mathcal{F}(B), p_{2}(C)=q_{1}\left(C_{*}\right)$. Then $p_{2}(B)=q_{1}\left(B_{*}\right)=q_{1}(A \backslash F)=1$. Further, if $C_{1}, C_{2}, \ldots$ is a sequence of mutually disjoint sets in $\mathcal{F}(B)$, then $C_{1 *}, C_{2 *}, \ldots$ are mutually disjoint sets in $\mathcal{F}(A)$. So,

$$
\sum_{k} p_{2}\left(C_{k}\right)=\sum_{k} q_{1}\left(C_{k *}\right)=q_{1}\left[\bigcup_{k} c_{k *}\right]=q_{1}\left[\left(\bigcup_{k} c_{k}\right)_{*}\right]=p_{2}\left(\bigcup_{k} C_{k}\right) .
$$

Hence, $p_{2}$ is a probability measure on $\mathcal{F}(B)$.

C. We show that $<p_{1}, p_{2}>\left(W^{2}\right) \geqslant 1-\varepsilon$. Notice that

$$
\mathrm{U}=\{(\mathrm{a}, \mathrm{F} \cup\{a\}): \mathrm{a} \in \mathrm{A} \backslash \mathrm{F}\} \quad \text { and } \quad \mathrm{V}=\{(\mathrm{c}, \mathrm{F} \cup\{\mathrm{a}\}): \mathrm{c} \in \mathrm{F} \text { and } \mathrm{a} \in \mathrm{A} \backslash \mathrm{F}\}
$$

are subsets of $W^{2}$. Thus, it suffices to show that $\left\langle p_{1}, p_{2}>(U \cup V) \geqslant 1-\varepsilon\right.$.

C1. We first argue that

$$
<\mathrm{p}_{1}, \mathrm{p}_{2}>(\mathrm{U} \cup \mathrm{V})=<\mathrm{p}_{1}, \mathrm{p}_{2}>(\mathrm{U})+<\mathrm{p}_{1}, \mathrm{p}_{2}>(\mathrm{V}) .
$$


Clearly

$$
<\mathrm{p}_{1}, \mathrm{p}_{2}>(\mathrm{U} \cup \mathrm{V}) \leqslant<\mathrm{p}_{1}, \mathrm{p}_{2}>(\mathrm{U})+<\mathrm{p}_{1}, \mathrm{p}_{2}>(\mathrm{V})
$$

We show the reverse inequality. Notice that $V=F \times L$. Let $\left\{Z^{i}\right\}_{i \in \mathbb{N}}$ be a cover of $U \cup V$. Let $Z^{i}=Z_{1}^{i} \times Z_{2}^{i}$ be a rectangle in the cover. Define $G_{1}^{i}=Z_{1}^{i} \cap F$, $H_{1}^{i}=Z_{1}^{i} \backslash F$, and $H_{2}^{i}=Z_{2}^{i} \cap L$.

Notice that $F \in \mathcal{F}(A)$, and $L=Q(F,|F|+1) \in \mathcal{F}(B)$. So, $G_{1}^{i} \in \mathcal{F}(A), H_{1}^{i} \in \mathcal{F}(A)$, and $\mathrm{H}_{2}^{\mathrm{i}} \in \mathcal{F}(\mathrm{B})$.

Moreover, $\mathrm{G}_{1}^{\mathrm{i}} \times \mathrm{H}_{2}^{\mathrm{i}}$ and $\mathrm{H}_{1}^{\mathrm{i}} \times \mathrm{H}_{2}^{i}$ are disjoint subsets of $\mathrm{Z}^{i}$. Also, if $(a, F \cup$ $\{a\}) \in U$, then $(a, F \cup\{a\}) \in H_{1}^{i} \times H_{2}^{i}$. If $(a, F \cup\{a\}) \in V$, then $(a, F \cup\{a\}) \in$ $\mathrm{G}_{1}^{\mathrm{i}} \times \mathrm{H}_{2}^{\mathrm{i}}$.

C2. We show that $\left\langle p_{1}, p_{2}>(V)=(1-d) \cdot(1-\varepsilon)\right.$. We already observed that $V=F \times L$. Moreover, $F \in \mathcal{F}(A)$, and $L=Q(F,|F|+1) \in \mathcal{F}(B)$. So,

$$
\begin{aligned}
<p_{1}, p_{2}>(V) & =p_{1}(F) \cdot p_{2}(L)=p_{1}(F) \cdot q_{1}(A \backslash F) \\
& =(1-d) \cdot\left[\sum_{a \in F} c_{a}\right] \cdot 1 \geqslant(1-d) \cdot(1-\varepsilon) .
\end{aligned}
$$

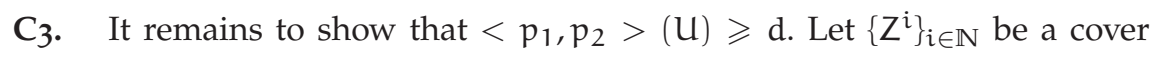
of $U=\{(a, F \cup\{a\}): a \in A \backslash F\}$. Let $Z^{i}=Z_{1}^{i} \times Z_{2}^{i}$ be a rectangle in the cover. Define

$$
Y_{1}^{i}=\left\{a \in A \backslash F:(a, F \cup\{a\}) \in Z^{i}\right\} \quad \text { and } \quad Y_{2}^{i}=\left\{F \cup\{a\}: a \in Y_{1}^{i}\right\}
$$

Clearly, $Y_{1}^{i}$ is a subset of $Z_{1}^{i}$ and $Y_{2}^{i}$ is a subset of $Z_{2}^{i}$.

Claim. It holds that $Y_{2}^{i} \in \mathcal{F}(B)$ and $Y_{1}^{i} \in \mathcal{F}(A)$.

We first show that $Y_{2}^{i} \in \mathcal{F}(B)$. Write $J_{2}^{i}=\left\{F \cup\{a\}: a \in Z_{1}^{i}\right\}$. Then $Y_{2}^{i}=J_{2}^{i} \backslash\{F\}$. Thus, by condition [B3], it suffices to show that $J_{2}^{i} \in \mathcal{F}(B)$. We do this in two steps.

Step 1. If $J_{2}^{i}$ is countable. Then $J_{2}^{i} \in \mathcal{F}(B)$ by condition [B3], plus the observation that $\mathcal{F}(\mathrm{B})$ is a sigma-algebra.

Step 2. If $J_{2}^{i}$ is not countable. Then $Z_{1}^{i}$ is not countable. So, since $Z_{1}^{i} \in$ $\mathcal{F}(A)$ and $\mathcal{F}(A)$ is the minimal sigma-algebra on $A$, this implies that $A \backslash Z_{1}^{i}$ is countable. So, $D=\left\{F \cup\{a\}: a \notin Z_{1}^{i}\right\}$ is countable, and hence an element of $\mathcal{F}(B)$. Then either $J_{2}^{i}=L \backslash D$ or $J_{2}^{i}=(L \backslash D) \cup\{F\}$. In either case, since $\mathcal{F}(B)$ is 
a sigma-algebra, and $L=Q(F,|F|+1)$ is an element of $\mathcal{F}(B), J_{2}^{i}$ is an element of $\mathcal{F}(B)$. Now note that

$$
Y_{2 *}^{i}=\left\{a \in A: F \cup\{a\} \in Y_{2}^{i}\right\}=\left\{a \in A \backslash F: a \in Y_{1}^{i}\right\}=Y_{1}^{i} .
$$

It follows that $Y_{1}^{i}=Y_{2 *}^{i} \in \mathcal{F}(A)$.

C4. Define $Y^{i}=Y_{1}^{i} \times Y_{2}^{i}$. Clearly the sets $Y^{i}$ cover $U$. Thus, it suffices to show that

$$
\sum_{i \in \mathbb{N}} p_{1}\left(Y_{1}^{i}\right) \times p_{2}\left(Y_{2}^{i}\right) \geqslant d
$$

For a set $Y^{i}=Y_{1}^{i} \times Y_{2}^{i}$,

$$
\left\langle p_{1}, p_{2}\right\rangle\left(Y^{i}\right)=p_{1}\left(Y_{1}^{i}\right) \cdot p_{2}\left(Y_{2}^{i}\right) \geqslant d \cdot q_{1}\left(Y_{1}^{i}\right) \cdot q_{1}\left(Y_{2 *}^{i}\right)=d \cdot q_{1}\left(Y_{1}^{i}\right) \cdot q_{1}\left(Y_{1}^{i}\right) .
$$

Further, since the sets $Y^{i}$ cover $U$, the sets $Y_{1}^{i}$ cover $A \backslash F$. Then there is a $k$ with $q_{1}\left(Y_{1}^{k}\right)>0$. Then however, since $A$ is an atom of $q_{1}$, this implies that $\mathrm{q}_{1}\left(\mathrm{Y}_{1}^{\mathrm{k}}\right)=1$.

Finally, since $v_{1}^{p}=0$, all probability measure in $\Delta(A)$ are pessimistic optimal for player 1 . This completes the proof.

4.B TOOLBOX

In this section we briefly discuss two well-known theorems needed in our arguments.

Lemma 4.B.1. Let $p \in \Delta(S)$. Then there are

[1] a countable set $\mathrm{I}$

[2] coefficients $\mathrm{c} \geqslant 0$ and $\mathrm{c}_{\mathrm{i}} \geqslant 0$ for all $i \in \mathrm{I}$, with $\mathrm{c}+\sum_{i \in \mathrm{I}} \mathrm{c}_{i}=1$, and

[3] a non-trivial probability measure $\mathrm{q}$ on $\mathrm{S}$ and Dirac measures $\delta_{s_{i}}$ on $s_{i} \in \mathrm{S}$ for every $i \in I$

such that $\mathrm{p}=\mathrm{c} \cdot \mathrm{q}+\sum_{\mathrm{i} \in \mathrm{I}} \mathrm{c}_{\mathrm{i}} \cdot \delta_{s_{i}}$.

Proof. Let $I=\{s \in S: p(\{s\})>0\}$. Note that $I$ is countable. Define $c_{i}=$ $p(\{i\})$, for all $i \in I$, and $c=1-\sum_{i \in I} c_{i}$. If $c>0$, then $q(T)=p(T \backslash I) / c$, for all $\mathrm{T} \subseteq \mathrm{S}$.

The following theorem has been proved by Sierpiński (1922). 
Theorem 4.B.2. Let $(\mathrm{S}, \mathcal{F}, \mathrm{p})$ be an atomless probability measure space. For any $\mathrm{t} \in(0,1)$ there exists a set $\mathrm{U} \in \mathcal{F}$ for which $\mathrm{p}(\mathrm{U})=\mathrm{t}$.

Proof. The proof is in two parts.

A. Let $V \in \mathcal{F}$ with $p(V)>0$ be given. Take $c$ and d such that $2 c \leqslant d<p(V)$. We show that there is a set $W \in \mathcal{F}$ such that $W \subseteq V$ and $c \leqslant p(W) \leqslant d$.

If $c=0$, we take $W=\emptyset$. Suppose $c>0$. Let $\mathcal{V}$ be the collection of sets $C \in \mathcal{F}$ with $C \subseteq$ V. Define

$$
s=\sup \{p(C): p(C)<c, C \in \mathcal{V}\}
$$

Note that $s \leqslant c$. Since $S$ does not have atoms, $s>0$. Take a sequence $\left(C_{k}\right)_{k \in \mathbb{N}}$ in $\mathcal{V}$ where $p\left(C_{k}\right) \rightarrow s$. Let $D_{m}=\cup_{k=1}^{m} C_{k}$. Suppose there is an $m$ with $p\left(D_{m}\right) \geqslant c$. Let $n$ be the first such $m$. Then $p\left(D_{n}\right) \leqslant 2 c \leqslant d$. So, we can take $W=D_{n}$.

Suppose alternatively that $p\left(D_{m}\right)<c$ for all $m$. Define $D=\cup_{\mathfrak{n} \in \mathbb{N}} D_{n}$. It follows from monotone convergence that $p(D)=s \leqslant c$. Suppose that $p(D)=$ c. Then we can take $W=D$.

Suppose alternatively that $p(D)<c$. Then, since $p$ is atomless, there is a set $E \subseteq V \backslash D$ with $0<p(E)<c-p(D)$. It then follows that $s<p(E \cup D)<c$. This contradicts the definition of $s$.

B. Now take any $t \in(0,1)$. We show that there exists a set $U \in \mathcal{F}$ for which $p(U)=t$. Since $S$ is not an atom, there is a $U_{1} \in \mathcal{F}$ such that $\frac{t}{2} \leqslant p\left(U_{1}\right) \leqslant t$.

Suppose $U_{n}$ is defined with $p\left(U_{n}\right) \leqslant t$ and $t-p\left(U_{n}\right) \leqslant \frac{t-p\left(U_{1}\right)}{2^{n-1}}$. If $p\left(U_{n}\right)=$ $\mathrm{t}$, we take $\mathrm{U}=\mathrm{U}_{\mathrm{n}}$. Otherwise, take $\mathrm{c}=\frac{\mathrm{t}-\mathrm{p}\left(\mathrm{U}_{\mathrm{n}}\right)}{2}$ and $\mathrm{d}=\mathrm{t}-\mathrm{p}\left(\mathrm{U}_{\mathrm{n}}\right)$. By the previous argument applied to $V=S \backslash U_{n}$, there is a $W \in \mathcal{F}$ and $W \subseteq V$ such that $\frac{t-p\left(U_{n}\right)}{2} \leqslant p(W) \leqslant t-p\left(U_{n}\right)$. Let $U_{n+1}=U_{n} \cup W$. Then $p\left(U_{n+1}\right)=$ $p\left(U_{n}\right)+p(W) \leqslant t$ and $t-p\left(U_{n+1}=t-p\left(U_{n}\right)-p(W) \leqslant \frac{t-p\left(U_{n}\right)}{2} \leqslant\right.$ $\frac{\mathrm{t}-\mathrm{p}\left(\mathrm{u}_{1}\right)}{2^{\mathrm{n}}}$.

If the iterative procedure does not terminate, let $U=\cup_{n \in \mathbb{N}} U_{n}$. Then by monotone convergence, $p(U)=t$. 
In this section we briefly define and discuss the set theoretic axioms that feature in this chapter.

Zermelo Fraenkel The Zermelo Fraenkel axiom system is the universally accepted axiom system on which modern mathematics is built. We do not venture into all its separate axioms, but only observe that the ZF axiom system is conservative, in the sense that it does not include controversial axioms, and it is therefore widely accepted.

Axiom of Choice Let I be any non-empty set, and suppose that for every $i \in I, V_{i}$ is a non-empty set. Write

$$
V=\bigcup_{i \in I} V_{i}
$$

A function $f: I \rightarrow V$ with $f(i) \in V_{i}$ for every $i \in I$ is called a choice function. The Axiom of Choice asserts the following.

AC For every choice of sets $I$ and $V_{i}$, a choice function $f: I \rightarrow V$ exists.

Axiom of Determinacy Let $A$ be a subset of $\mathbb{N}^{\mathbb{N}}$. This set $A$ defines a game $G(A)$ in the following way. There are two players. The players take turns to choose elements of $\mathbb{N}$. The game has perfect information, so that players observe each other's choice. The game is then played as follows.

Player 1 chooses $n_{1}$,

player 2 chooses $n_{2}$,

player 1 chooses $n_{3}$,

player 2 chooses $n_{4}$,

and so on. This way a sequence

$$
s=\left(n_{1}, n_{2}, n_{3}, n_{4}, \ldots\right) \in \mathbb{N}^{\mathbb{N}}
$$

is constructed. Player 1 wins the game $G(A)$ if $s \in A$, otherwise player 2 wins. The game $G(A)$ is called determined if either player 1 or player 2 has a winning strategy. The Axiom of Determinacy states the following.

AD For every set $A \subseteq \mathbb{N}^{\mathbb{N}}$, the resulting game $G(A)$ is determined.

The Axiom of Determinacy implies the continuum hypothesis. 
Continuum Hypothesis We say that two sets $X$ and $Y$ have the same cardinality if there exists a one-to-one and onto $f: X \rightarrow Y$.

$\mathrm{CH}$ Let $X$ be an infinite subset of the unit interval $[0,1]$. Then either $X$ has the cardinality of $\mathbb{N}$, or $X$ has the cardinality of $\mathbb{R}$.

Generalized Continuum Hypothesis Let $A$ be a non-empty set. A binary relation on $A$ is a subset of $A \times A$. Let $\preceq$ be a binary relation on $A$. When $x \preceq y$ and not $y \preceq x$ we write $x \prec y$. Let $Y$ be a subset of $A$. An element $y \in Y$ is called the smallest element of $Y$ when $y \prec z$ for all $z \in Y$ with $z \neq y$. The binary relation $\preceq$ is called a well-ordering when $\preceq$ satisfies

[1] ( reflexivity) for all $x \in X$ we have $x \preceq x$,

[2] ( transitivity) for all $x, y, z \in X$ we have $x \preceq z$ whenever both $x \preceq y$ and $y \preceq z$,

[3] ( totality) for all $x, y \in X$ with $x \neq y$ we have $x \prec y$ or $y \prec x$, and

[4] ( well order) every non-empty subset of $A$ has a smallest element.

A set $A$ is called transitive when for every $a \in A$ it holds that $a \subseteq A$. A set $A$ is called an ordinal number when $A$ is transitive and moreover $A$ is well-ordered by set inclusion. For example,

$$
A=\{\emptyset,\{\emptyset\},\{\emptyset,\{\emptyset\}\}\}
$$

is an ordinal number. The set $a=\{\emptyset,\{\emptyset\}\}$ is both an element and a subset of A.

It can be shown that, for any two ordinal numbers $\alpha$ and $\beta$, either $\alpha \in \beta$ or $\beta \in \alpha$. Conversely, each element of an ordinal number is an ordinal number itself.

When two sets $X$ and $Y$ have the same cardinality, we write $X \sim Y$. If there is a one-to-one map $f: X \rightarrow Y$, we write $X \leqslant Y$. The Theorem of Cantor-BernsteinSchroeder shows that $X \leqslant Y$ and $Y \leqslant X$ imply $X \sim Y$. We write $X<Y$ when $X \leqslant Y$ and not $X \sim Y$.

An ordinal number $\mathrm{k}$ is called a cardinal number if there does not exist an ordinal number $\alpha \in \mathrm{k}$ with $\alpha \sim \mathrm{k}$. For every cardinal number $\mathrm{k}$, there exists a smallest cardinal number $\mu$ with $\kappa<\mu$. We write $\mu=\kappa^{+}$. 
Now assume that the Axiom of Choice is true. Then for every set $X$ there is a cardinal number $k$ such that $X \sim \kappa$. We define $\operatorname{Card}(X)=\kappa$. The generalized continuum hypothesis states the following.

GCH For every cardinal number $\mathrm{k}, \operatorname{Card}\left(2^{\kappa}\right)=\kappa^{+}$.

Measurable Cardinals Let $\mathrm{k}$ be a cardinal number. A binary map on $\mathrm{k}$ is a map $\mu: 2^{\kappa} \rightarrow\{0,1\}$. A binary map on $\kappa$ is called $\kappa$-additive if for every cardinal number $\lambda \in \mathrm{K}$ and for every mutually disjoint collection $\left(A_{\xi}\right)_{\xi \in \lambda}$ of sets $A_{\xi} \subseteq \kappa$ it holds that

$$
\mu\left(\bigcup_{\xi \in \lambda} A_{\xi}\right)=\sum_{\xi \in \lambda} \mu\left(A_{\xi}\right) .
$$

A cardinal number $\mathrm{k}$ is called measurable if there exists a binary $\mathrm{k}$-additive binary map on $\kappa$ with $\mu(\kappa)=1$ and $\mu(\{\lambda\})=0$ for all $\lambda \in \kappa$.

MC There exists a measurable cardinal.

Constructable sets and $\mathrm{V}=\mathrm{L} \quad$ The statement $\mathrm{V}=\mathrm{L}$ is called the Axiom of Constructability. Its formulation and its associated contructions are due to Gödel. Let A be a set. An expression $\phi$ is called a formula for A if

[1] $\phi$ has one free variable $x$

[2] the only predicate symbols in $\phi$ are " $\in$ " and " $="$

[3] the individual-constants in $\phi$ are names of elements of $A$

[4] the quantifiers are restricted to $A$. So, it only uses $\forall y \in A$ and $\exists y \in A$.

Let $A$ be a set, and let $B$ be a subset of $A$. We say that $B$ is a definable subset of $A$ when there is a formula $\phi$ for $A$ such that

$$
B=\{x \in A: \phi(x)\} .
$$

We define for every set $A$

$$
\operatorname{Def}(A)=\{B \subseteq A: B \text { is a definable set of } A\} .
$$

Define $L(\phi)=\{\phi\}$. Using transfinite induction, for every ordinal $\alpha$ we define

$$
\mathrm{L}(\alpha)=\bigcup_{\beta \in \alpha} \operatorname{Def}(\mathrm{L}(\beta))
$$


We say that a set $B$ is constructable (by abuse of notation denoted by $B \in L$ ) if there is an ordinal $\alpha$ with $B \in L(\alpha)$. The class $L$ is the collection of all constructable sets.

$\mathrm{V}=\mathrm{L} . \quad$ Every set is constructable. 

FINITELY ADDITIVE MIXED AND BEHAVIOR

STRATEGIES IN INFINITE DURATION DECISION

PROBLEMS

\subsection{INTRODUCTION}

In the previous chapters we considered two-player simultaneous-move games. In this chapter we consider one-player infinite-length games, so decision problems. In the previous chapters we focused on games with infinite action spaces. If the action spaces are finite, then in Chapter 4 player 2 wins, and in Chapter 3 we arrive at the same analysis as von Neumann (1928). In this chapter we also consider decision problems with finite action spaces.

OUR SETUP We consider the following decision problem. Let $A$ denote a set of actions, having at least two elements, and let $P$ denote the set of all infinite sequences of elements of $A$. Let $u: P \rightarrow \mathbb{R}$ denote a bounded payoff function. At every period $t=1,2, \ldots$, the decision maker chooses an action $a_{t} \in A$, knowing his previously chosen actions $a_{1}, \ldots, a_{t-1}$, i.e., the history. The decision maker's payoff is given by $u\left(a_{1}, a_{2}, \ldots\right)$.

We study this problem in the finitely additive setup. That is, probability measures are only assumed to be finitely additive, and not necessarily countably additive. Our goal is to examine and compare expected payoffs induced by mixed and behavior strategies in these decision problems.

StRAtegies A pure strategy is a function that specifies an action at each history. A mixed strategy is a charge on the set of pure strategies, with the interpretation that the decision maker should choose a pure strategy according to this charge.

A behavior strategy gives recommendations to the decision maker in an essentially different fashion. A behavior strategy prescribes a charge on the action space at each history. The interpretation is that, at each history that 
arises during the play of the decision problem, the decision maker should choose an action according to the corresponding charge.

INDUCED CHARges on $2^{P}$ AND ExPECted PAyoff Given a strategy, it is natural to ask which distribution it induces on the the set $\mathrm{P}$ of all infinite sequences of actions, since the payoff function $u$ is defined on P. Once we know the answer to this question, we can calculate the induced expected payoff by simply taking the integral of the payoff function $u$ with respect to this charge.

For pure strategies the answer is easy. Consequently, we can define the induced charge on $2^{\mathrm{P}}$ for every mixed strategy in a natural way.

For behavior strategies, the question is more challenging, both from a conceptual and from a technical point of view. We discuss four different approaches to this problem. In each approach, we first fix an algebra $\mathscr{P}$ on the set $\mathrm{P}$ and then define for each behavior strategy which charge it induces on this algebra. One could think of $\mathscr{P}$ as the collection of sets on which we think that each behavior strategy should induce a certain unique probability.

Then, given such an algebra $\mathscr{P}$ of $\mathrm{P}$, we say that a mixed strategy and a behavior strategy are equivalent whenever they generate the same charge on the algebra $\mathscr{P}$.

OUR CONTRIBUTION

[1] We start with arguably the smallest-least restrictive-algebra that one could consider. Then, step by step we enlarge the algebra (cf. Section 5.6).

I. The first algebra that we consider is the algebra consisting of all events that only depend on the first $n$ actions, for some $n \in \mathbb{N}$. This is a very natural algebra.

II. The second, and generally larger, algebra consists of all events such that it eventually becomes certain whether the event occurs or not. It turns out that this algebra coincides with the collection of all clopen subsets of $P$, where clopen stands for closed and open with respect to a natural topology. This algebra is examined in detail in Dubins and Savage (2014).

III. Note that singletons of P do not belong to the second algebra, and hence we cannot express the probability of a certain sequence $\left(a_{1}, a_{2}, \ldots\right)$ with respect to any behavior strategy. Thus the third algebra that we consider is the 
smallest algebra that includes the second algebra and all singletons of P. A subset of $\mathrm{P}$ belongs to this algebra exactly when it differs from a clopen set by only a finite number of elements of $P$.

IV. The largest algebra that we analyse is the algebra induced by all open sets. This algebra was already investigated by Dubins (1974). It is richer than the one considered under Approach III, but is still smaller than the Borel sigma-algebra.

[2] We compare these four approaches. In particular, we prove that each approach is consistent with all earlier approaches. This means the following. Given an approach, a behavior strategy induces a charge on the algebra corresponding to the approach. When we restrict this charge to an algebra corresponding to one of the earlier approaches, then it coincides with the charge induced by the behavior strategy under that approach (cf. (13), Lemmas 5.6.7 and 5.6.10).

[3] With respect to the first and second approaches, we provide sufficient and necessary topological conditions on the payoff function under which each behavior strategy induces a unique expected payoff. Similarly, we provide sufficient conditions with respect to the third and fourth approaches (cf. Theorem 5.7.2).

[4] With respect to all approaches, we prove that each behavior strategy has an equivalent mixed strategy. We show that with respect to the third and fourth approaches the converse does not hold, and in each decision problem there is a mixed strategy that does not have an equivalent behavior strategy (cf. Theorem 5.7.1).

[5] We provide several illustrative examples that clarify the differences between the four approaches.

STRUCTURE OF THE CHAPTER In Section 5.2 we introduce the model and define strategies, and in Section 5.3 we illustrate the problem with the help of examples. In Section 5.4 we define the topology, which will play an important role in our investigation and in our main results. In Section 5.5 we discuss induced charges by mixed and behavior strategies. In Section 5.6 we discuss the four approaches connected to behavior strategies. In Section $5 \cdot 7$ 
we present our main results. The proofs are given in Appendices 5.A and 5.B. In Section 5.8 we conclude with some remarks.

RELATED LITERATURE

Finite additivity For related literature on finitely additivity probabilities, see Chapter 1. In addition, for related literature on finitely additive strategies in decision theory, see Chapter 3.

Dubins and Savage (2014) study the same type of decision problems as we do, and mention what we call Approach I, but focus on Approach II. Dubins (1974) considers what we call Approach IV. Purves and Sudderth (1976) go further, and consider the Borel sigma-algebra, which is larger than the algebras that we cover in our four approaches.

Sudderth (2016) writes about finitely additive dynamic programming. His model includes finitely additive strategies and an infinite horizon, as our model does. However, his model has states and probabilistic transitions, and the payoff is some type of aggregation of daily payoffs, for example by discounting. The paper also contains comparisons to the countably additive theory. For example, it shows that there are cases when there exists a finitely additive optimal stationary strategy, in contrast, a countably additive one need not exist.

Equivalent strategies Equivalence between mixed and behavior strategies has been investigated in finite games with perfect recall, see Kuhn's theorem in Maschler et al (2013), Theorem 6.15. For infinite-length games with finite action spaces, see Theorem 6.26 in Maschler et al (2013) or Takahashi (1969). Muraviev et al (2017) extend Kuhn's result to Ellsberg games. Aryal and Stauber (2014) show the limits of extending Kuhn's theorem in the case of ambiguity averse players (for more about ambiguity, see Chapter 3).

\subsection{THE MODEL}

the Decision problem Let $A$ be an action set, having at least two elements. Let $\mathrm{H}$ denote the set of finite sequences in $A$, including the empty sequence $\varnothing$. Let $P$ denote the set of infinite sequences in $A$. Elements of $A$ are called actions, elements of $\mathrm{H}$ are called histories and elements of $\mathrm{P}$ are called plays. Let $u: \mathrm{P} \rightarrow \mathbb{R}$ be a bounded function, called the payoff function. 
Consider the following decision problem. At each period $t=1,2, \ldots$, the decision maker chooses ${ }^{1}$ an action $a_{t}$ from $A$, knowing his previous choices $\left(a_{1}, \ldots, a_{t-1}\right) \in H$. This induces a play $p=\left(a_{1}, a_{2}, \ldots\right)$. The payoff of the decision maker is $u(p)$.

A strategy is a decision rule that gives a recommendation to the decision maker how to choose his actions during the decision problem. These recommendations can be given in essentially different ways, and based on that we distinguish three different types of strategies: pure strategies, mixed strategies and behavior strategies. We discuss each type of strategies in turn.

PURE StRAtegies A pure strategy is a function $f: H \rightarrow A$. The interpretation is that if history $h$ arises during the decision problem, then the strategy $f$ recommends the decision maker to choose action $f(h)$. Each pure strategy $f$ induces a unique play $p_{f} .{ }^{2}$ We denote the set of pure strategies by $F$.

MIXED STRATEGIES A mixed strategy $m$ is a charge on the set of pure strategies, i.e., $m \in \mathcal{C}\left(F, 2^{F}\right)$. The interpretation is that the decision maker should draw a pure strategy according to $m$, and play the decision problem by using f.

BEHAVIOR STRATEgies A behavior strategy is a function $\mathrm{b}: \mathrm{H} \rightarrow \mathrm{C}\left(\mathrm{A}, 2^{\mathrm{A}}\right)$. The interpretation is that if history $h$ arises during the decision problem, then the strategy $b$ recommends the decision maker to choose an action according to the charge $b(h)$. As we will see, in our finitely additive setting, these strategies are the most difficult to handle. The reason is that a behavior strategy may involve successive uses of charges during the decision problem, possibly even infinitely many of them.

In this chapter we assume the Axiom of Choice.

\section{$5 \cdot 3$ EXAMPLES}

In this section we discuss a number of illustrative and thought-provoking examples, with the intention to focus on the intuition and the main ideas.

1 Note that the set of available actions does not depend on the history of the decision problem, and is always $A$.

2 We say that two pure strategies that induce the same play are outcome-equivalent. 
The precise formal arguments and technical details are delayed to Section $5 \cdot 7$.

The first example is essentially a two-period decision problem.

Example 5.3.1. Consider the decision problem with action space $A=\mathbb{N}$ and the following payoff function: for a play $p=\left(a_{1}, a_{2}, \ldots\right) \in P$, if $a_{1}=a_{2}=n$ for some $n \in \mathbb{N}$ then $\mathfrak{u}(p)=\frac{n}{n+1}$ and otherwise $u(p)=0$. So the payoff is determined after the second period. Intuitively, the decision maker would like to choose a large action at period 1 and repeat the same action at period 2.

In this decision problem, there is no pure strategy that gives payoff 1. Yet, the decision maker can obtain an expected payoff of 1 by playing a mixed strategy, which can be constructed as follows. For every $n \in \mathbb{N}$, let $f_{n}$ be a pure strategy which chooses action $n$ at periods 1 and 2 . Let $\tau$ be a pure charge on $\mathbb{N}$. Intuitively, let $m$ be the mixed strategy that prescribes to choose $n \in \mathbb{N}$ according to $\tau$ and then to play the pure strategy $f_{n}$. Formally, for every $G \subseteq F$, we let $m(G)=\tau\left(\left\{n \in \mathbb{N}: f_{n} \in G\right\}\right)$.

Since $\tau$ is a pure charge on $\mathbb{N}$, the decision maker can expect to draw a large natural number $n$, i.e. for all $N \in \mathbb{N}$

$$
\tau(\{1, \ldots, N\})=0 \quad \text { and } \quad m\left(\left\{f_{1}, \ldots, f_{N}\right\}\right)=0
$$

and

$$
\tau(\{\mathrm{N}+1, \mathrm{~N}+2, \ldots\})=1 \quad \text { and } \quad \mathrm{m}\left(\left\{\mathrm{f}_{\mathrm{N}+1}, \mathrm{f}_{\mathrm{N}+2}, \ldots\right\}\right)=1 .
$$

The mixed strategy $m$ induces an expected payoff of 1 , i.e. by (6)

$$
\int_{n \in \mathbb{N}} u\left(f_{n}\right) \tau(d n)=1 .
$$

Note that all countably additive mixed strategies induce an expected payoff strictly less than 1.

Now consider a corresponding behavior strategy $\mathrm{b}$ such that $\mathrm{b}$ chooses an action according to $\tau$ at period 1 and repeats this action at period 2 . That is, $b(\varnothing)=\tau$ and $b(n)=\delta_{n}$ for every $n \in \mathbb{N}$, where $\delta_{n}$ is the Dirac measure on action $n$. The payoff $\frac{n}{n+1}$ is received on the set of plays that start with action $n$ at periods 1 and 2. Naturally, we calculate the expected payoff under 
the behavior strategy $b$ by the iterative integral over the first two periods as follows:

$$
\begin{gathered}
\int_{a_{1} \in A} \int_{a_{2} \in A} u\left(a_{1}, a_{2}, \ldots\right) b\left(a_{1}\right)\left(d a_{2}\right) b(\varnothing)\left(d a_{1}\right) \\
=\int_{a_{1} \in A} \frac{a_{1}}{a_{1}+1} b(\varnothing)\left(d a_{1}\right)=\int_{a_{1} \geqslant n} \frac{a_{1}}{a_{1}+1} b(\varnothing)\left(d a_{1}\right) \geqslant \frac{n}{n+1},
\end{gathered}
$$

for all $n \in \mathbb{N}$. The second equality follows from (6). Therefore $b$ induces an expected payoff of 1 . All of our approaches have this type of finite iterative integration as part of them.

The next example is a variation on Example 5.3.1.

Example 5.3.2. Consider the decision problem with action space $A=\mathbb{N}$ and the following payoff function: for a play $p=\left(a_{1}, a_{2}, \ldots\right) \in P$, if $a_{1}=\ldots=$ $a_{n}=n$ for some $n \in \mathbb{N}$ then $u(p)=\frac{n}{n+1}$ and otherwise $u(p)=0$. If action $\mathrm{n}$ is played at period 1 , then the payoff is determined after period $n$. Consequently, the payoff is determined in finite but unbounded time. Intuitively, the decision maker would like to choose a large number $n$ and play action $n$ at periods $1, \ldots, n$.

Similarly to Example 5.3.1, there is no pure strategy that gives payoff 1, but one can construct a mixed strategy that induces an expected payoff of 1 . For every $n \in \mathbb{N}$, let $f_{n}$ be a pure strategy which chooses action $n$ at periods $1, \ldots, n$. Let $\tau$ be a pure charge on $\mathbb{N}$. For every $G \subseteq F$, we let $m(G)=$ $\tau\left(\left\{n \in \mathbb{N}: f_{n} \in G\right\}\right)$.

Let $\mathrm{b}$ denote the behavior strategy for which $\mathrm{b}(\varnothing)$ is a pure charge on $\mathbb{N}$, but for any other $h=\left(a_{1}, \ldots, a_{t}\right) \in H$ the probability distribution $b(h)$ is simply the Dirac measure on action $a_{1}$. Let $R_{n}$ denote the set of plays that start with action $n$ at the first $n$ periods. If we want to calculate the probability of $R_{n}$ under b, as in Example 5.3.1, then we need to use $n$ iterative integrals. So in contrast with Example 5.3.1, with a finite but bounded number of iterative integrals, we cannot conclude that the set $\cup_{n} \geqslant N R_{n}$ has probability 1 , for an $\mathrm{N} \in \mathbb{N}$.

This example illustrates the main difference between our first and second approaches. In our first approach, which is based on finite but bounded iterative integrals, $\mathrm{b}$ does not induce a unique expected payoff. However in the second 
approach, by considering stop rules (Definition 5.4.2) that identify histories where the payoff is determined, we will find that $b$ induces an expected payoff of 1. A similar example is shown in Dubins and Savage (2014), Section 2.6.

The next two examples in this section are stopping decision problems. In these decision problems the decision maker has two actions, one of which could be interpreted as "continue" and the other as "stop". The payoff is determined by the first period when the decision maker plays the latter action.

Example 5.3.3. Consider the decision problem with action space $A=\{c, s\}$. Denote $p=(c, c, \ldots)$. Let the payoff function $u$ be given by $u(p)=1$ and $u\left(p^{\prime}\right)=0$ for all $p^{\prime} \in P \backslash\{p\}$.

The pure strategy that always chooses action $c$ induces payoff 1 , and so does the mixed strategy that places probability 1 on this pure strategy.

From now on, we focus on the corresponding behavior strategy $b$ that chooses action $c$ with probability 1 at every history, i.e., $b(h)(c)=1$ for every $h \in H$. Let $h_{t}$ denote the history at period $t$ in which only action $c$ is played. Then, at every period $t$, the history $h_{t}$ has probability 1 and the history $h_{t-1} s$ has probability 0 . Notice that

$$
\bigcup_{t \in \mathbb{N}}\left[h_{t-1} s\right]=P \backslash\{p\},
$$

where $\left[h_{t-1} s\right]$ denotes the set of plays that start with prefix $h_{t-1} s$ up to period $t$.

In the context of countably additive probabilities, this would imply that $P \backslash\{p\}$ has probability 0 , and hence that $\{p\}$ has probability 1 . Thus in that context, $\mathrm{b}$ would yield an expected payoff of 1 .

In our model with charges, this cannot be concluded without additional assumptions. In our first two approaches, with fairly minimal assumptions, the algebras are small and do not contain the singleton $\{p\}$, and any probability on $\{p\}$ is consistent under the behavior strategy $b$. That is, with respect to the first two approaches, $b$ does not induce a unique expected payoff. The third and fourth approaches consider larger algebras which do contain $\{p\}$ 
and assign probability 1 to $\{\mathrm{p}\}$ under $\mathrm{b}$. Under these approaches the expected payoff induced by $b$ is 1 .

Example 5.3.4. Consider the decision problem with action space $A=\{c, s\}$ and the following payoff function: for a play $p \in P$, if action $s$ is played at period $t$ for the first time then $u(p)=\frac{t}{t+1}$, and otherwise, if $p$ only contains action $c$ then $u(p)=0$.

In this decision problem, there is no pure strategy that gives payoff 1. Yet, similarly to Example 5.3.1, the decision maker can obtain an expected payoff of 1 by playing a mixed strategy, which can be constructed as follows. For every $n \in \mathbb{N}$, let $f_{n}$ be a pure strategy that prescribes action $c$ at all periods before $n$ and action $s$ at period $n$. Let $\tau$ be a pure charge on $\mathbb{N}$. Let the mixed strategy $m$ be defined by $m(G)=\tau\left(\left\{n \in \mathbb{N}: f_{n} \in G\right\}\right)$ for all $G \subseteq F$. Thus intuitively, $m$ prescribes to choose $n \in \mathbb{N}$ according to $\tau$ and then to play the pure strategy $f_{n}$. This means that if the decision maker plays according to $m$, then he eventually plays $s$ with probability 1 , but the probability that he plays $s$ in the first $T$ periods is 0 , for every $T \in \mathbb{N}$. This strategy induces an expected payoff of 1 . Note that all countably additive mixed strategies induce an expected payoff strictly less than 1 .

In the class of behavior strategies, the interesting question is which expected payoff the behavior strategy $b$ gives that always chooses action $c$ with probability 1 . The analysis is identical to the same behavior strategy in Example 5.3.3, and it depends on further assumptions which expected payoffs we obtain in the interval $[0,1]$.

Example 5.3.5. Consider the decision problem with action space $A=\mathbb{N}$ and the following payoff function: for any $n \in \mathbb{N}$, the play $p=(n, 1,1, \ldots)$ has a payoff of $\mathfrak{u}(p)=\frac{n}{n+1}$, and otherwise the payoff is $u(p)=0$.

In this decision problem, there is no pure strategy that gives payoff 1. Yet, similarly to Example 5.3.1, the decision maker can obtain an expected payoff of 1 by playing a mixed strategy, which can be constructed as follows. For every $n \in \mathbb{N}$, let $f_{n}$ be a pure strategy that prescribes action $n$ at period 1 , and at all other periods it prescribes 1 . Let $\tau$ be a pure charge on $\mathbb{N}$. Let the mixed strategy $m$ be defined by $m(G)=\tau\left(\left\{n \in \mathbb{N}: f_{n} \in G\right\}\right)$ for all $G \subseteq F$. This strategy induces an expected payoff of 1 . 
Take the behavior strategy $b$ that assigns to the root $\tau$, so $b(\varnothing)=\tau$, and at every other history $h \in H$ it assigns the Dirac measure on action 1, so $b(h)=\delta_{1}$. The expected payoff induced by $b$ is only unique under the fourth approach.

\section{$5 \cdot 4$ TOPOLOGICAL ISSUES}

For $n \in \mathbb{N}$, a history $h \in A^{n}$ and a period $T \leqslant n, T \in \mathbb{N}$ we denote by $h(T)$ the action taken at period $T$ by history $h$. Similarly, for a play $p$ we denote by $p(T)$ the action in $p$ taken at period $T$. We use the notation $h \prec p$, if $p(t)=h(t)$ for all $t \leqslant n$ and $t \in \mathbb{N}$. Similarly, for an $m \in \mathbb{N}$ and a history $h^{\prime} \in A^{m}$, we use the notation $h \preceq h^{\prime}$, if $h(t)=h^{\prime}(t)$ for all $t \leqslant n$, and use the notation $h \prec h^{\prime}$, if $h \preceq h^{\prime}$ and $h \neq h^{\prime}$.

We endow $\mathrm{A}$ with the discrete topology and $\mathrm{P}$ with the induced product topology denoted by $\mathcal{T}$. Note that $P$ is compact if and only if $A$ is finite, and whenever $\mathrm{P}$ is compact, it is homeomorphic to the Cantor ternary set. Furthermore, $(P, \mathcal{T})$ is completely metrizable. We consider the metric $d: P \times$ $P \rightarrow \mathbb{R}$ which is defined as follows: if $p=q$ then $d(p, q)=0$, and otherwise $d(p, q)=2^{-k(p, q)}$ where $k(p, q) \in \mathbb{N}$ is the least period at which $p$ and $q$ differ.

In $(P, \mathcal{T})$, a sequence of plays $\left(p_{n}\right)_{n \in \mathbb{N}}$ converges to a play $p$ if for every $k \in \mathbb{N}$ there exists an $N_{k} \in \mathbb{N}$ such that for every $n \geqslant N_{k}$ the first $k$ coordinates of $p_{n}$ coincide with those of $p$. For more details on the topological space $(P, \mathcal{T})$, we refer to Kechris (1995). The same topology is used in Dubins and Savage (2014).

A function $\mathrm{g}: \mathrm{P} \rightarrow \mathbb{R}$ is said to be continuous at a play $\mathrm{p} \in \mathrm{P}$ if for every sequence of plays $\left(p_{\mathfrak{n}}\right)_{\mathfrak{n} \in \mathbb{N}}$ converging to $p$, we have $\lim _{\mathfrak{n} \rightarrow \infty} g\left(p_{\mathfrak{n}}\right)=g(p)$. A function $\mathrm{g}: \mathrm{P} \rightarrow \mathbb{R}$ is said to be continuous if $\mathrm{g}$ is continuous at every play $p \in P$.

A function $g: \mathrm{P} \rightarrow \mathbb{R}$ is said to be uniformly continuous if for every $\varepsilon>0$ there exists $\delta>0$ such that for every $p, q \in P$ with $d(p, q)<\delta$ we have $|g(p)-g(q)|<\varepsilon$. For example, the payoff function in Example 5.3.1 is uniformly continuous (the payoff is determined after the first two periods). The 
payoff function in Example 5.3.2 is continuous, but not uniformly continuous. Clearly, if $\mathrm{g}$ is uniformly continuous, then $\mathrm{g}$ is also continuous. If $\mathrm{A}$ is finite, then by compactness of $\mathrm{P}$ the converse also holds.

The following lemma follows directly from the definition of the metric $d$.

Lemma 5.4.1. Consider a function $\mathrm{g}: \mathrm{P} \rightarrow \mathbb{R}$.

1. $\mathrm{g}$ is continuous at a play $\mathrm{p} \in \mathrm{P}$ if and only if for every $\varepsilon>0$, there is a $\mathrm{T} \in \mathbb{N}$ such that if for a play $\mathrm{q}$ it holds that $\mathrm{q}(\mathrm{t})=\mathrm{p}(\mathrm{t})$ for all $\mathrm{t} \leqslant \mathrm{T}$, then $|g(p)-g(q)|<\varepsilon$.

2. $\mathrm{g}$ is uniformly continuous if and only if for every $\varepsilon>0$, there is $a \mathrm{~T} \in \mathbb{N}$ such that if for two plays $\mathrm{p}$ and $\mathrm{q}$ it holds that $\mathrm{p}(\mathrm{t})=\mathrm{q}(\mathrm{t})$ for all $\mathrm{t} \leqslant \mathrm{T}$, then $|g(p)-g(q)|<\varepsilon$.

Stop rules will play an important role in our definitions.

Definition 5.4.2. A stop rule is a set $S \subseteq H$ for which there is no $h, h^{\prime} \in S$ with $h \prec h^{\prime}$. A stop rule $S$ is horizontal if there is an $n \in \mathbb{N}$ such that $S \subseteq A^{n}$. A stop rule $S$ is complete if for every play $p \in P$ there is a history $h \in S$ with $h \prec p$.

The definitions of stop rules and complete stop rules are related to Sections 2.9 and 2.11 in Dubins and Savage (2014). However, there is a slight difference in phrasing. What they call an incomplete stop rule, we simply call a stop rule, and what they call a stop rule, we call a complete stop rule.

We show the formal connection between the incomplete stop rules of Dubins and Savage (2014) and our stop rules. In Dubins and Savage (2014) an incomplete stop rule $s$ is a function $s: P \rightarrow \mathbb{N} \cup\{\infty\}$. Let $P^{\prime}=\{p \in P: s(p)<\infty\}$. In our model a corresponding stop rule $S \subseteq \mathrm{H}$ can be defined by $S=\{\mathrm{h} \in \mathrm{H}$ : $\exists p \in P^{\prime}$ with $h \prec p$ and $\left.h \in A^{s(p)}\right\}$.

Take a stop rule $S$. Associated with $S$ we define the set

$$
[S]=\{p \in P: \exists h \in S: h \prec p\}
$$

of plays. It is straightforward to check that $[S]$ is open. In particular, for every history $h \in H$,

$$
[h]=\{p \in P: h \prec p\} \in \mathcal{T} .
$$


A set of the form $[h]$ is called a cylinder set. Note that, $[S]=\bigcup_{h \in S}[h]$.

The next lemma characterizes continuous and uniformly continuous functions on $\mathrm{P}$ by complete stop rules. The proof follows directly from the definitions.

Lemma 5·4·3. Consider a function $\mathrm{g}: \mathrm{P} \rightarrow \mathbb{R}$.

1. $g$ is continuous if and only if for every $\varepsilon>0$, there is a complete stop rule $\mathrm{S} \subseteq \mathrm{H}$ such that, for all histories $\mathrm{h} \in \mathrm{S}$ and all plays $\mathrm{p} \succ \mathrm{h}$ and $\mathrm{q} \succ \mathrm{h}$, we have $|u(p)-u(q)|<\varepsilon$.

2. $\mathrm{g}$ is uniformly continuous if and only if for every $\varepsilon>0$, there is a complete horizontal stop rule $\mathrm{S}=\mathrm{A}^{\mathrm{n}}$, for some $\mathrm{n} \in \mathbb{N}$, such that for all histories $\mathrm{h} \in \mathrm{S}$ and all plays $\mathrm{p} \succ \mathrm{h}$ and $\mathrm{q} \succ \mathrm{h}$, we have $|\mathrm{u}(\mathrm{p})-\mathrm{u}(\mathrm{q})|<\varepsilon$.

Consider a function $\mathrm{g}: \mathrm{P} \rightarrow \mathbb{R}$. We say that $\mathrm{g}$ is upper semi-continuous if for every $r \in \mathbb{R}$ the set $g^{-1}([r, \infty))$ is closed. Equivalently, for every play $p$ and every sequence of plays $p_{n}$ converging to $p$ we have $\limsup _{n \rightarrow \infty} g\left(p_{n}\right) \leqslant$ $g(p)$. Similarly, we say that $g$ is lower semi-continuous if for every $r \in \mathbb{R}$ the set $\mathrm{g}^{-1}((-\infty, r])$ is closed. Equivalently, for every play $\mathrm{p}$ and every sequence of plays $p_{n}$ converging to $p$ we have $\liminf _{n \rightarrow \infty} g\left(p_{n}\right) \geqslant g(p)$. A function is continuous if and only if it is both upper and lower semi-continuous.

Consider a function $\mathrm{g}: \mathrm{P} \rightarrow \mathbb{R}$. We say that $\mathrm{g}$ has a limit at $\mathrm{p} \in \mathrm{P}$ if there is $\ell \in \mathbb{R}$ with the following property: $g\left(p_{n}\right)$ converges to $\ell$ for each sequence $p_{n}$ in $P$ such that (1) $p_{n}$ converges to $p$ as $n \rightarrow \infty$, and (2) $p_{n} \neq p$ for all $n \in \mathbb{N}$. If the limit at $p$ exists, we denote it by $L_{g}(p)$. Note that the function $g$ is continuous at $p$ if and only if $g$ has a limit at $p$ and $L_{g}(p)=g(p)$.

Consider a function $\mathrm{g}: \mathrm{P} \rightarrow \mathbb{R}$ and a play $\mathrm{p} \in \mathrm{P}$. The oscillation of $\mathrm{g}$ at $\mathrm{p}$ is defined as

$$
\mathrm{o}_{g}(p)=\lim _{\varepsilon \downarrow 0} \sup _{q, r \in \mathcal{N}_{\varepsilon}(p)}|g(q)-g(r)|,
$$

where $\mathcal{N}_{\varepsilon}(p)=\{q \in P: d(p, q)<\varepsilon\}$ is the $\varepsilon$-neighbourhood of $p$. Note that a function $g$ is continuous at $p$ if and only if $o_{g}(p)=0$.

We call a function $g: P \rightarrow \mathbb{R}$ weakly tame if for every $r>0$ the set $\{p \in P$ : $\left.o_{g}(p) \geqslant r\right\}$ is finite. A continuous function is clearly weakly tame. 
Consider a function $\mathrm{g}: \mathrm{P} \rightarrow \mathbb{R}$. Let $\mathrm{D}_{\mathrm{g}}$ be the set of plays at which $\mathrm{g}$ is not continuous. We say that a discontinuity $p \in \mathrm{D}_{\mathrm{g}}$ is removable if $\mathrm{g}$ has a limit at $p \in \mathrm{D}_{\mathrm{g}}$. We call the function $\mathrm{g}$ strongly tame if the following two conditions hold:

1. The function $\mathrm{g}$ is weakly tame.

2. Each discontinuity in $\mathrm{D}_{g}$ is removable.

The proof can be found in Appendix 5.A.

Lemma 5.4.4. Consider a function $\mathrm{g}: \mathrm{P} \rightarrow \mathbb{R}$. Assume that each discontinuity in $\mathrm{D}_{\mathrm{g}}$ is removable. Then the function $\mathrm{L}_{\mathrm{g}}: \mathrm{P} \rightarrow \mathbb{R}$ is continuous.

The following example provides an illustration of strongly tame functions.

Example 5.4.5. Consider the action space $A=\mathbb{N}$. For every $n \in A$ let $p_{n}$ be the play where action $n$ is played at all periods and let $P_{n}$ be the set of plays that start with action $n$. Let the function $g$ be as follows: for a play $p \in P_{n}$ we set $g(p)=1$ if $p=p_{n}$ and $g(p)=\frac{n}{n+1}$ if $p \in P_{n} \backslash\left\{p_{n}\right\}$. The function $g$ is continuous everywhere except at the plays $p_{1}, p_{2}, \ldots$

The function $g$ is strongly tame. First, each discontinuity $p_{n}$ is removable, and $o_{g}\left(p_{n}\right)=\frac{1}{n+1}$. Second, for any $r>0$, the set

$$
\left\{p \in P: o_{g}(p) \geqslant r\right\}=\left\{p_{n}: n \leqslant \frac{1-r}{r}\right\}
$$

is clearly finite.

Counterexample. Consider the following function $g^{\prime}$ : for a play $p \in P_{n}$ we set $g^{\prime}(p)=1$ if $p=p_{n}$ and $g^{\prime}(p)=0$ if $p \in P_{n} \backslash\left\{p_{n}\right\}$. This function is not weakly tame, as for every $r \in(0,1)$ the set

$$
\left\{p \in P: o_{g}(p) \geqslant r\right\}=\left\{p_{n}: n \in \mathbb{N}\right\}
$$

is infinite.

\subsection{INDUCED CHARges ON THE SET P OF PLAys AND EXPECTED PAy- OFFS}

In this section we consider the charges on the set $\mathrm{P}$ of plays induced by strategies. Note that the strategies are defined on the corresponding power 
sets. More concretely, mixed strategies are defined on $\left(F, 2^{F}\right)$, so the power set of pure strategies. Given a history, a behavior strategy is defined on $\left(A, 2^{A}\right)$, so the power set of actions. We are interested in what charge these strategies induce on the the set $\mathrm{P}$ of plays. We will see that a mixed strategy induces a charge on the power set of plays. However, it is not clear what charge a behavior strategy induces on the power set of plays, so our starting point is to look at what charge it induces on different algebras on the set of plays.

\subsubsection{Mixed strategies}

In this subsection we consider mixed strategies and examine the induced charges on the set of plays and the corresponding expected payoffs.

A pure strategy $f$ induces a unique play $p_{f}$. Define $U(f)=u\left(p_{f}\right)$. As a mixed strategy $m$ prescribes to draw a pure strategy $f$ according to the charge $m$ defined on $\left(\mathrm{F}, 2^{\mathrm{F}}\right)$, the expected payoff for the mixed strategy $m$ is given by

$$
U(m)=\int_{f \in F} U(f) m(d f) .
$$

In a natural way, a mixed strategy $m$ generates a charge $m^{*}$ on $\left(P, 2^{P}\right)$, given for every $\mathrm{Q} \subseteq \mathrm{P}$ by

$$
m^{*}(Q)=m\left(\left\{f \in F: p_{f} \in Q\right\}\right) .
$$

Thus, the probability of $\mathrm{Q}$ according to $\mathrm{m}^{*}$ is equal to the probability according to $m$ that a pure strategy is chosen which induces a play in the set Q.

Note that, as different pure strategies can induce the same play, different mixed strategies can generate the same charge on $\left(\mathrm{P}, 2^{\mathrm{P}}\right)$.

The next lemma establishes a natural relationship between the expected payoff for a mixed strategy and the induced charge on $\left(\mathrm{P}, 2^{\mathrm{P}}\right)$. The proof is given in Appendix 5.A.

Lemma 5.5.1. For every mixed strategy $m$

$$
u(m)=\int_{p \in P} u(p) m^{*}(d p) .
$$


Note that a pure strategy can be seen as a mixed strategy. Indeed, if $f$ is a pure strategy and $\delta_{f}$ is the Dirac measure on $f$, then $\left(\delta_{f}\right)^{*}$ is the Dirac measure on $p_{f}$ and $U\left(m_{f}\right)=U(f)$.

\subsubsection{Behavior strategies}

In this subsection we consider behavior strategies and describe the induced charges on the set of plays and the corresponding expected payoffs.

For a behavior strategy it is not immediately evident what the corresponding charge on the set $\mathrm{P}$ of plays should be. Consequently, since the expected payoff induced by a behavior strategy is the integral of the payoff function with respect to the induced charge on $\mathrm{P}$, it is not evident what the expected payoff for a behavior strategy should be either.

ALgebras on the Set P of plays and specifications In the next section we discuss in total four different approaches to the above mentioned problem. In each approach, we first fix an algebra on the set $\mathrm{P}$ of plays and then define for each behavior strategy which charge it induces on $(\mathrm{P}, \mathscr{P})$. One should think of $\mathscr{P}$ as the collection of sets $\mathrm{Q} \subseteq \mathrm{P}$ on which we agree that a behavior strategy $\mathrm{b}$ should induce a certain unique probability, which is denoted by $\psi^{\mathscr{P}}(\mathrm{b})(\mathrm{Q})$.

Arguably, a minimal requirement on the algebra $\mathscr{P}$ is that it contains all cylinder sets $[S]$ where $S$ is a stop rule and $S \subseteq A^{n}$ for some $n \in \mathbb{N}$. Let $\mathcal{R}^{\text {hs }}$ denote the set of all such sets. The superscript hs refers to horizontal stop rules.

Lemma 5.5.2. $\mathcal{R}^{\text {hs }}$ is an algebra.

The proof is given in Appendix 5.A.

Take a behavior strategy $b$ and a set $[S]$, where $S \subseteq A^{n}$ for some $n \in \mathbb{N}$. The probability of the set $[S]$ under $b$ can be calculated through $n$ iterated integrals as follows: let $\mathrm{c}^{\text {hs }}(\mathrm{b})([\mathrm{S}])=$

$$
\int_{a_{1} \in A} \cdots \int_{a_{n} \in A} \mathbb{I}_{S}\left(a_{1}, \ldots, a_{n}\right) b\left(a_{1}, \ldots, a_{n-1}\right)\left(d a_{n}\right) \cdots b(\varnothing)\left(d a_{1}\right) .
$$

Note that $c^{\text {hs }}(b)$ is a charge on the algebra $\mathcal{R}^{\text {hs }}$. 
Definition 5.5.3. An algebra $\mathscr{P}$ of $\mathrm{P}$ is called competent if $\mathscr{P} \supseteq \mathcal{R}^{\mathrm{hs}}$.

Given a competent algebra $\mathscr{P}$, a $\mathscr{P}$-specification is a function $\psi^{\mathscr{P}}$ that to each behavior strategy $\mathrm{b}$ assigns a charge $\psi^{\mathscr{P}}(\mathrm{b})$ on $(\mathrm{P}, \mathscr{P})$ with the requirement that for all sets $[R] \in \mathcal{R}^{\text {hs }}$

$$
\psi^{\mathscr{P}}(\mathrm{b})([R])=\mathrm{c}^{\mathrm{hs}}(\mathrm{b})([\mathrm{R}]) .
$$

Note that $\mathcal{R}^{\text {hs }}$, in view of Lemma 5.5.2, is a competent algebra of $\mathrm{P}$ and $c^{\text {hs }}$ is an $\mathcal{R}^{\text {hs }}$-specification.

For a competent algebra $\mathscr{P}$ and a $\mathscr{P}$-specification $\psi^{\mathscr{P}}$, and for a given behavior strategy $b$, the charge $\psi^{\mathscr{P}}$ (b) can be extended, generally not uniquely, from the algebra $\mathscr{P}$ to all subsets of P. The set of these charges is denoted by $<\mathrm{b}\left|\mathscr{P}, \psi^{\mathscr{P}}\right\rangle$. For more details on this extension, see Appendix 3.C.

Consequently, a behavior strategy $\mathrm{b}$ generally induces a set of possible expected payoffs. Indeed, for each $\mathrm{B} \in<\mathrm{b} \mid \mathscr{P}, \psi^{\mathscr{P}}>$, we obtain an expected payoff

$$
u(B)=\int_{p \in P} u(p) B(d p) .
$$

Let

$$
\mathrm{u}\left(\mathrm{b} \mid \mathscr{P}, \psi^{\mathscr{P}}\right)=\left\{\mathrm{u}(\mathrm{B}): \mathrm{B} \in<\mathrm{b} \mid \mathscr{P}, \psi^{\mathscr{P}}>\right\} .
$$

equivalence of strategies Consider a competent algebra $\mathscr{P}$ on P. By equation (8), each mixed strategy $m$ induces a charge $\mathrm{m}^{*}$ on $(\mathrm{P}, \mathscr{P})$. Given a $\mathscr{P}$-specification $\left(\mathrm{P}, \psi^{\mathscr{P}}\right)$, each behavior strategy $\mathrm{b}$ also induces a charge $\psi^{\mathscr{P}}$ (b) on $\mathscr{P}$. This allows us to define equivalence between strategies.

Definition 5.5.4. Let $\mathscr{P}$ denote a competent algebra of $\mathrm{P}$, and let $\psi^{\mathscr{P}}$ be a $\mathscr{P}$-specification. A mixed strategy $\mathrm{m}$ and a behavior strategy $\mathrm{b}$ are said to be $\mathscr{P}$-equivalent if they generate the same charge on the algebra $(\mathrm{P}, \mathscr{P})$, i.e., $\mathrm{m}^{*}(\mathrm{Q})=\psi^{\mathscr{P}}(\mathrm{b})(\mathrm{Q})$ for every set $\mathrm{Q} \in \mathscr{P}$.

Given an algebra $\mathscr{P}$, we say that the payoff function $u$ is uniformly $\mathscr{P}$ approachable, if for every $\varepsilon>0$ there exists a $\mathscr{P}$-measurable simple-function $u^{\prime}: P \rightarrow \mathbb{R}$ such that $\left|\mathfrak{u}(p)-\mathfrak{u}^{\prime}(p)\right| \leqslant \varepsilon$ for every $p \in P$. We consider a different algebra $\mathscr{P}$ for each approach. Marinacci (1997) in Section 4 and Harris et 
al (2005) in Section 2.1.3 focus on uniformly $\mathscr{P}$-approachable functions, however they work with a different model, namely one-shot simultaneous-move games. For more on their work, see Sections 3.1 and 3.4.3.

UNIQUe expected payoffs We already know from equation (7) that a mixed strategy induces a unique expected payoff. The following lemma identifies a sufficient condition for the uniqueness of expected payoffs under behavior strategies. The proof can be found in Appendix 5.A.

Theorem 5.5.5. Let $\mathscr{P}$ denote a competent algebra of $\mathrm{P}$, and let $\psi^{\mathscr{P}}$ be a $\mathscr{P}$ specification. Suppose that the payoff function $\mathrm{u}$ is uniformly $\mathscr{P}$-approachable. Then, for every behavior strategy $\mathrm{b}$ the set $\mathrm{u}\left(\mathrm{b} \mid \mathscr{P}, \psi^{\mathscr{P}}\right)$ is a singleton. This means that $\mathrm{b}$ induces a unique payoff.

The opposite direction of the above theorem does not hold, as shown in Example 5.7.3.

\subsection{Four approaches to induce Charges on the Set P of Plays BY BEHAVIOR STRATEGIES}

In the next subsections we discuss in turn the four approaches to the choices of the algebra $\mathscr{P}$ and the $\mathscr{P}$-specification $\psi^{\mathscr{P}}$. The first approach considers the minimal competent algebra $\mathcal{R}^{\text {hs }}$ on $\mathrm{P}$, and then the subsequent algebras are getting larger step by step.

\subsubsection{Approach I: the minimal competent algebra $\mathcal{R}^{\text {hs }}$}

In this subsection, we further investigate the minimal competent algebra $\mathcal{R}^{\text {hs }}$ and the corresponding $\mathcal{R}^{\text {hs }}$-specification $c^{\text {hs }}$, which was defined in (9). Let $\psi^{\text {hs }}=c^{\text {hs }}$. Dubins and Savage (2014) mention a similar idea in Section 2.6, but find it insufficient for their purpose.

The next lemma claims that $\mathcal{R}^{\text {hs }}$ is strongly related to the set CL of all clopen (closed and open) subsets of P. The proof can be found in Appendix 5.A.

Lemma 5.6.1. The algebra $\mathcal{R}^{\text {hs }}$ has the following properties:

1. $\mathcal{R}^{\text {hs }} \subseteq \mathrm{CL}$. 
2. $\mathcal{R}^{\text {hs }}=\mathrm{CL}$ if and only if the action set $\mathrm{A}$ is finite.

For each behavior strategy $b$, let $u^{\text {hs }}(b)$ denote the set of possible expected payoffs, i.e. $\mathrm{U}^{\mathrm{hs}}(\mathrm{b})=\mathrm{U}\left(\mathrm{b} \mid \mathcal{R}^{\mathrm{hs}}, \psi^{\text {hs }}\right)$, as defined in (II).

5.6.2 Approach II: stop rules

In this section, we define the algebra $\mathcal{R}^{s}$ on $\mathrm{P}$ and a corresponding $\mathcal{R}^{s}$ specification $\psi^{s}$. The superscript $s$ refers to stop rules. The main idea of this approach is to look at subsets of P such that they and their complements can be defined through stop rules.

Let

$$
\mathcal{S}=\{[S]: S \subseteq H \text { is a stop rule }\} .
$$

Thus, $\mathcal{S}$ consists of all subsets of $P$ that are induced by a stop rule. We define

$$
\mathcal{R}^{S}=\{[R]:[R] \in \mathcal{S} \text { and } P \backslash[R] \in \mathcal{S}\} .
$$

The following lemma summarizes basic properties of $\mathcal{S}$ and $\mathcal{R}^{s}$. The proof can be found in Appendix 5.A. The lemma is connected to Theorem 2.9.1 in Dubins and Savage (2014).

Lemma 5.6.2. The collections $\mathcal{S}$ and $\mathcal{R}^{s}$ have the following properties:

1. $\mathcal{S}$ is equal to the set of all open sets in $\mathrm{P}$.

2. $\mathcal{R}^{s}$ is an algebra.

3. $\mathcal{R}^{s}$ is equal to the set of all clopen sets of $\mathrm{P}$.

4. $\mathcal{R}^{\mathrm{s}} \supseteq \mathcal{R}^{\mathrm{hs}}$, and hence $\mathcal{R}^{\mathrm{s}}$ is competent.

5. $\mathcal{R}^{s}=\mathcal{R}^{\text {hs }}$ if and only if the action set $\mathrm{A}$ is finite.

For Approach II we need the definition of a subproblem. Given a decision problem $G, k \in \mathbb{N}$, a history $h \in A^{k}$ and a payoff function $u$, we denote the subproblem that starts at history $h$ by $\left.G\right|_{h}$. This is played as follows. At periods $n \geqslant k+1$, the decision maker chooses an action $a_{n} \in A$, which induces a play $\left(a_{k+1}, a_{k+2}, \ldots\right)$ and a corresponding payoff $\left.u\right|_{h}\left(a_{k+1}, a_{k+2}, \ldots\right)=$ 
$u\left(h, a_{k+1}, a_{k+2}, \ldots\right)$. In fact, $\left.G\right|_{h}$ is identical to the decision problem $G$, except for the payoff function (and for the inessential change that the first period has label $k+1)$. For a $k \in \mathbb{N}$ and for a history $h \in A^{k}$, let

$$
\left.\mathrm{Q}\right|_{\mathrm{h}}=\left\{\left(\mathrm{q}_{\mathrm{k}+1}, \mathrm{q}_{\mathrm{k}+2}, \ldots\right):\left(\mathrm{h}, \mathrm{q}_{\mathrm{k}+1}, \mathrm{q}_{\mathrm{k}+2}, \ldots\right) \in \mathrm{Q}\right\} .
$$

Note that $\left.\mathrm{Q}\right|_{h} \in \mathcal{R}^{s}$. The behavior strategy $\mathrm{b}$ in decision problem $\mathrm{G}$ induces a behavior strategy $\left.b\right|_{h}$ in the subproblem $\left.G\right|_{h}$ as follows: $\left.b\right|_{h}\left(\left.h^{\prime}\right|_{h}\right)=b\left(h^{\prime}\right)$ for any $h^{\prime} \succeq h$. That is, $\left.b\right|_{h}\left(a_{k+1}, a_{k+2}, \ldots, a_{k^{\prime}}\right)=b\left(h, a_{k+1}, a_{k+2}, \ldots, a_{k^{\prime}}\right)$, for every $k^{\prime} \geqslant k$. Note that $\left.b\right|_{h}$, as a mapping from $H$ to $\mathcal{C}\left(A, 2^{A}\right)$, is a behavior strategy itself according to our definition.

An $\mathcal{R}^{s}$-specification $\psi$ defines an extension $\psi(b)$ in decision problem $G$, and for a history $h \in H$ the $\mathcal{R}^{s}$-specification $\psi$ defines an extension $\psi\left(\left.b\right|_{h}\right)$ in decision problem $\left.\mathrm{G}\right|_{h}$. The following lemma claims that there is a unique $\mathcal{R}^{s}$-specification $\psi$ with a fairly natural consistency property between the probabilities under $\psi\left(\left.b\right|_{h}\right)$ and $\psi\left(\left.b\right|_{h a}\right)$, for each behavior strategy $b$, history $h$ and action a. More precisely, for every set in $\mathcal{R}^{s}$, the corresponding probability under $\psi\left(\left.b\right|_{h}\right)$ should be equal to the expectation of the probability at the next period under $\psi\left(\left.b\right|_{h a}\right)$. The proof of the lemma is given in Appendix 5.A. The following lemma is strongly related to Theorem 2.8.1 from Dubins and Savage (2014), even though the proofs have a different style. Theorem 1 in Purves and Sudderth (1976) is also similar.

Lemma 5.6.3. There exists a unique $\mathcal{R}^{\mathrm{s}}$-specification $\psi$ with the following property: for every behavior strategy $b$, for every history $h$ and for every $Q \in \mathcal{R}^{s}$

$$
\psi\left(\left.b\right|_{h}\right)\left(\left.Q\right|_{h}\right)=\int_{a \in A} \psi\left(\left.b\right|_{h a}\right)\left(\left.Q\right|_{h a}\right) b(h)(d a) .
$$

Let $\psi^{\mathrm{s}}$ be the unique $\mathcal{R}^{\mathrm{s}}$-specification in Lemma 5.6.3. Note that by the definition of an $\mathcal{R}^{s}$-specification, we have for every behavior strategy $b$ and every $[R] \in \mathcal{R}^{\text {hs }}$ that

$$
\psi^{s}(b)([R])=\psi^{h s}(b)([R]),
$$

which also intuitively states that Approach II is consistent with Approach I. The following lemma further examines the relation of Approach II to Approach I. It claims that, if the action space is infinite, then for some behavior 
strategies $b$, the charge $\psi^{s}(b)$ is not a unique extension of $\psi^{\text {hs }}(b)$. That is, Approach II is not the only way to extend Approach I to $\mathcal{R}^{s}$. The proof of the lemma can be found in Appendix 5.A.

Lemma 5.6.4 (Approach I and Approach II are different). Assume that the action space $\mathrm{A}$ is infinite. Then there is a behavior strategy $\mathrm{b}$ and a charge $\mu$ on $\mathcal{R}^{s}$ such that for every $[\mathrm{S}] \in \mathcal{R}^{\text {hs }}$

$$
\psi^{s}(b)([S])=\mu([S]),
$$

but $\psi^{\mathrm{s}}(\mathrm{b}) \neq \mu$.

In the above lemma, it is necessary to assume that $A$ is infinite, in view of part 5 of Lemma 5.6.2.

For each behavior strategy $b$, let $\mathrm{U}^{\mathrm{s}}(\mathrm{b})$ denote the set of possible expected payoffs, i.e. $U^{s}(b)=U\left(b \mid \mathcal{R}^{s}, \psi^{s}\right)$, as defined in (11). Theorem 2.8.4 in Dubins and Savage (2014) is comparable to finding a singleton $\mathrm{U}^{\mathrm{s}}(\mathrm{b})$.

\subsubsection{Approach III: stop rules and singletons}

In this section, we define an algebra $\mathcal{R}^{s+s}$ on $\mathrm{P}$ and a corresponding $\mathcal{R}^{s+s_{-}}$ specification $\psi^{s+s}$. The superscript $s+s$ refers to stop rules and singletons. The main idea of this approach is to extend the algebra $\mathcal{R}^{s}$ from Approach II by singleton plays.

Let $\mathcal{R}^{s+s}$ be the smallest algebra that contains all sets from $\mathcal{R}^{s}$ and all singletons $\{p\}$, where $p \in P$. Note that $\mathcal{R}^{s+s}$ is competent by part 4 of Lemma 5.6.2.

Lemma 5.6.5. The algebra $\mathcal{R}^{\mathrm{s}+\mathrm{s}}$ has the following properties:

1. $\mathcal{R}^{s+s}$ is a strict superset of $\mathcal{R}^{s}$, i.e. $\mathcal{R}^{s+s} \supsetneq \mathcal{R}^{s}$.

2. $\mathcal{R}^{s+s}$ is the collection of all subsets $\mathrm{Z}$ of $\mathrm{P}$ that differ in at most finitely many plays from a clopen set:

$$
\mathcal{R}^{s+s}=\left\{\mathrm{Z} \subseteq \mathrm{P}: \exists[\mathrm{R}] \in \mathcal{R}^{s} \text { such that both } \mathrm{Z} \backslash[\mathrm{R}] \text { and }[\mathrm{R}] \backslash \mathrm{Z} \text { are finite }\right\} .
$$

In other words, the algebra $\mathcal{R}^{s+s}$ is the collection of all subsets $\mathrm{Z}$ of $\mathrm{P}$ of the form

$$
\mathrm{Z}=\left([\mathrm{R}] \cup \mathrm{Q}_{1}\right) \backslash \mathrm{Q}_{2}
$$


where $[\mathrm{R}] \in \mathcal{R}^{\mathrm{s}}$ and $\mathrm{Q}_{1}$ and $\mathrm{Q}_{2}$ are finite subsets of $\mathrm{P}$.

3. For every $\mathrm{Z} \in \mathcal{R}^{s+s}$ there is a unique representation of the form (14) such that $[\mathrm{R}] \cap \mathrm{Q}_{1}=\emptyset, \mathrm{Q}_{1} \cap \mathrm{Q}_{2}=\emptyset$ and $[\mathrm{R}] \supseteq \mathrm{Q}_{2}$.

Take a behavior strategy $b$. Let $p=\left(a_{1}, a_{2}, \ldots\right) \in$ P. For each $n \in \mathbb{N}$, let

$$
l^{s+s}(b, p)=\prod_{n=1}^{\infty} b\left(a_{1}, \ldots, a_{n-1}\right)\left(a_{n}\right) .
$$

Take a $Z \in \mathcal{R}^{s+s}$. In view of Lemma 5.6.5, the set $Z$ can be written in a unique way of the form $Z=\left([R] \cup Q_{1}\right) \backslash Q_{2}$ such that $[R] \cap Q_{1}=\emptyset, Q_{1} \cap Q_{2}=\emptyset$ and $[R] \supseteq Q_{2}$. We define

$$
\psi^{s+s}(b)(Z)=\psi^{s}(b)([R])+\sum_{p \in Q_{1}} l^{s+s}(b, p)-\sum_{p \in Q_{2}} l^{s+s}(b, p) .
$$

This means that $l^{s+s}(b, p)$ is the limit of the probabilities that the behavior strategy $b$ induces on the histories $\left(a_{1}, \ldots, a_{n}\right)$. In a countably additive setup, $l^{s+s}(b, p)$ would be exactly the probability that $b$ induces the play $p$. Note that $\psi^{\text {hs }}(b)\left(\left[\left(a_{1}, \ldots, a_{k}\right)\right]\right)=$

$$
\psi^{s}(b)\left(\left[\left(a_{1}, \ldots, a_{k}\right)\right]\right)=\prod_{n=1}^{k} b\left(a_{1}, \ldots, a_{n-1}\right)\left(a_{n}\right),
$$

so $\psi^{\text {hs }}(b)\left(\left[\left(a_{1}, \ldots, a_{k}\right)\right]\right)$ and $\psi^{s}(b)\left(\left[\left(a_{1}, \ldots, a_{k}\right)\right]\right)$ converge to $l^{s+s}(b, p)$. One can verify that any representation of $Z$ from (14) gives the same outcome for $\psi^{s+s}$.

Lemma 5.6.6. Let $\mathrm{b}$ be a behavior strategy. The function $\psi^{s+s}(\mathrm{~b})$ is a charge on the algebra $\mathcal{R}^{\mathrm{s}+\mathrm{s}}$.

Notice that $\psi^{s+s}(b)([S])=\psi^{s}(b)([S])=\psi^{\text {hs }}(b)([S])$ for every behavior strategy $b$ and $[S] \in \mathcal{R}^{\text {hs }}$, because of (15) and by (13). Thus, $\psi^{s+s}$ is an $\mathcal{R}^{s+s}$ specification on the algebra $\mathcal{R}^{s+s}$.

The following lemma examines the relation of Approach III to Approach II, and is similar in spirit to (13) and Lemma 5.6.4. The proof is given in Appendix 5.A.

Lemma 5.6.7 (Approach II and Approach III are different). 
1. For every behavior strategy $\mathrm{b}$ and every $\mathrm{Z} \in \mathcal{R}^{\mathrm{s}}$

$$
\psi^{s+s}(b)(Z)=\psi^{s}(b)(Z)
$$

2. There is a behavior strategy $\mathrm{b}$ and a charge $\mu$ on $\mathcal{R}^{s+s}$ such that for every $\mathrm{Z} \in \mathcal{R}^{\mathrm{s}}$

$$
\psi^{s+s}(b)(Z)=\mu(Z),
$$

but $\psi^{s+s}(b) \neq \mu$.

We emphasize that the above lemma holds for any action space $A$ (under our assumption that $|A| \geqslant 2$ ), even when $A$ is finite.

For each behavior strategy $b$, let $\mathrm{U}^{\mathrm{s}+\mathrm{s}}(\mathrm{b})$ denote the set of possible expected payoffs, i.e. $\mathrm{U}^{\mathrm{s}+\mathrm{s}}(\mathrm{b})=\mathrm{U}\left(\mathrm{b} \mid \mathcal{R}^{\mathrm{s}+\mathrm{s}}, \psi^{\mathrm{s}+\mathrm{s}}\right)$, as defined in (II).

\subsubsection{Approach IV: open sets}

In this section, we consider an algebra $\mathcal{R}^{\mathrm{o}}$ on $\mathrm{P}$ and a corresponding $\mathcal{R}^{\mathrm{o}}$ specification $\psi^{\circ}$. The superscript o refers to open sets.

Let $\mathcal{R}^{\circ}$ be the smallest algebra of subsets of $\mathrm{P}$ that contains all open sets.

Lemma 5.6.8. $\mathcal{R}^{o}$ is a strict superset of $\mathcal{R}^{s+s}$, and $\mathcal{R}^{o}$ is a strict subset of the Borel sigma-algebra $\sigma(\mathrm{P})$ on $\mathrm{P}$. That is, $\mathcal{R}^{\mathrm{s}+\mathrm{s}} \subsetneq \mathcal{R}^{\mathrm{o}} \subsetneq \sigma(\mathrm{P})$.

Due to the first inclusion, $\mathcal{R}^{\mathrm{o}}$ includes the competent algebra $\mathcal{R}^{s+s}$, and hence $\mathcal{R}^{\mathrm{o}}$ is competent too. The second inclusion is not surprising as an algebra is only closed under countable unions under strong conditions. To provide a concrete example, in the proof we define a class $y$ of sets that all belong to $\sigma(\mathrm{P}) \backslash \mathcal{R}^{\mathrm{o}}$. A subset $\mathrm{Z}$ of $\mathrm{P}$ belongs to $\mathrm{y}$ if there is a non-empty open subset $\mathrm{O}$ of $\mathrm{P}$ with the following property: $\mathrm{Z}$ is dense in $\mathrm{O}$ and its complement $\mathrm{P} \backslash \mathrm{Z}$ is also dense in $\mathrm{O}$, i.e., $\mathrm{Z} \cap \mathrm{O}^{\prime} \neq \emptyset$ and $(\mathrm{P} \backslash \mathrm{Z}) \cap \mathrm{O}^{\prime} \neq \emptyset$ for every non-empty open set $\mathrm{O}^{\prime} \subseteq \mathrm{O}$.

Dubins (1974) considers the following probability of an open set based on inner approximations by clopen sets: for every behavior strategy $b$ and open set $\mathrm{O}$,

$$
c^{\mathrm{o}}(\mathrm{b})(\mathrm{O})=\sup _{\mathrm{Z} \in \mathcal{R}^{\mathrm{s}}, \mathrm{Z} \subseteq \mathrm{O}} \psi^{\mathrm{s}}(\mathrm{b})(\mathrm{Z})
$$


We will briefly investigate in Section 5.8 the counterpart of this definition by taking the outer approximations by clopen sets.

The following theorem is proven in Dubins (1974), Theorem 2.

Theorem 5.6.9. For every behavior strategy $\mathrm{b}$, the map $\mathrm{c}^{\mathrm{o}}(\mathrm{b})(\cdot)$ has a unique extension from the open subsets of $\mathrm{P}$ to a charge on the algebra $\mathcal{R}^{\circ}$.

Notice that $\psi^{\mathrm{o}}(\mathrm{b})([S])=\psi^{\mathrm{s}}(\mathrm{b})([S])=\psi^{\mathrm{hs}}(\mathrm{b})([\mathrm{S}])$ for every behavior strategy $\mathrm{b}$ and $[\mathrm{S}] \in \mathcal{R}^{\text {hs }}$, because of (17) and (13). This allows us to define the $\mathcal{R}^{\mathrm{o}}$ specification $\psi^{\circ}$ as follows: for every behavior strategy $b$, the charge $\psi^{\circ}(b)$ is the unique extension of $c^{o}(b)(\cdot)$ in Theorem 5.6.9.

The following lemma examines the relation of Approach IV to Approach III, and is similar in spirit to (13) and Lemmas 5.6.4 and 5.6.7. The proof is given in Appendix 5.A.

Lemma 5.6.10 (Approach III and Approach IV are different).

1. For every behavior strategy $\mathrm{b}$ and every $\mathrm{Z} \in \mathcal{R}^{\mathrm{s}+\mathrm{s}}$

$$
\psi^{o}(b)(Z)=\psi^{s+s}(b)(Z)
$$

2. Assume that the action space $\mathrm{A}$ is infinite. Then there is a behavior strategy $\mathrm{b}$ and a charge $\mu$ on $\mathcal{R}^{\circ}$ such that for every $\mathrm{Z} \in \mathcal{R}^{\mathrm{s}+\mathrm{s}}$

$$
\psi^{\mathrm{o}}(\mathrm{b})(\mathbf{Z})=\mu(\mathbf{Z})
$$

but $\psi^{\mathrm{o}}(\mathrm{b}) \neq \mu$.

For each behavior strategy $b$, let $\mathrm{U}^{\mathrm{o}}(\mathrm{b})$ denote the set of possible expected payoffs, i.e. $\mathrm{U}^{\mathrm{o}}(\mathrm{b})=\mathrm{U}\left(\mathrm{b} \mid \mathcal{R}^{\mathrm{o}}, \psi^{\mathrm{o}}\right)$, as defined in (II).

The algebra $\mathcal{R}^{0}$ is the largest algebra that we consider. As Example 5.6.11 shows, even using the algebra $\mathcal{R}^{o}$ and the corresponding specification $\psi^{\circ}$, not all behavior strategies generate a unique expected payoff. The following example is very similar to the example presented in Sections 4 and 5, Purves and Sudderth (1976).

Example 5.6.11. Consider a decision problem with action space $A=\left\{a, a^{\prime}\right\}$. Let $\mathrm{Q}$ be the set of plays $\mathrm{p}$ such that action a only appears finitely many times 
in $p$. We remark that $\mathrm{Q} \in \sigma(\mathrm{P}) \backslash \mathcal{R}^{\mathrm{o}}$, which is shown in the proof of Lemma 5.6.8, part B. Consider the following payoff function: $\mathfrak{u}(p)=1$ for every play $p \in Q$, and $\mathfrak{u}(p)=0$ otherwise. Take the behavior strategy $b$ which for every history $h \in H$ gives $b(h)(a)=\frac{1}{2}$ and $b(h)\left(a^{\prime}\right)=\frac{1}{2}$. The expected payoff depends on the probability of set $\mathrm{Q}$.

We show that $\mathrm{b}$ does not induce a unique expected payoff under Approach $\mathrm{IV}$, that is, $\mathrm{U}^{\mathrm{o}}(\mathrm{b})=\mathrm{U}\left(\mathrm{b} \mid \mathcal{R}^{\mathrm{o}}, \psi^{\mathrm{o}}\right)$ is not a singleton. For this purpose, we prove that $\psi^{\circ}(b)$ has two extensions from $\mathcal{R}^{\circ}$ to $2^{\mathrm{P}}, \mathrm{B}, \mathrm{B}^{\prime} \in<\mathrm{b} \mid \mathcal{R}^{\mathrm{o}}, \psi^{\mathrm{o}}>$ such that $u(B) \neq u\left(B^{\prime}\right)$, where we use the definition in (10). Since $u(B)=B(Q)$ and $u\left(B^{\prime}\right)=B^{\prime}(Q)$, we need to verify that $B(Q) \neq B^{\prime}(Q)$.

Note that $\psi^{\mathrm{o}}(\mathrm{b})(\{\mathrm{p}\})=0$ for every $p \in P$, because of part 1 of Lemma 5.6.10 and (15). As explained in the proof of Lemma 5.6.8, the sets $Q$ and $P \backslash Q$ are dense in all non-empty open subsets of $P$. So the inner and outer approximations of $\mathrm{Q}$ by sets in $\mathcal{R}^{\mathrm{o}}$ yield

$$
\begin{aligned}
& \sup _{\mathrm{Z} \in \mathcal{R}^{\circ}, \mathrm{z} \subseteq \mathrm{Q}} \psi^{\mathrm{o}}(\mathrm{b})(\mathrm{Z})=0, \\
& \inf _{\mathrm{Z} \in \mathcal{R}^{\circ}, \mathrm{Z} \supseteq Q^{\prime}} \psi^{\mathrm{o}}(\mathrm{b})(\mathrm{Z})=1 .
\end{aligned}
$$

Based on Theorem 3.C. 3 there exists an extension $B \in<b \mid \mathcal{R}^{\circ}, \psi^{\circ}>$ such that $\mathrm{B}(\mathrm{Q})=0$ and there exists an extension $\mathrm{B}^{\prime} \in<\mathrm{b} \mid \mathcal{R}^{\mathrm{o}}, \psi^{\mathrm{o}}>$ such that $\mathrm{B}^{\prime}(\mathrm{Q})=1$. The proof is complete.

We emphasize that the specification $\psi^{\circ}$ defines a charge on $\mathcal{R}^{\circ}$ for all behavior strategies. Given a specific behavior strategy b, Purves and Sudderth (1976) extend the charge $\psi^{\mathrm{o}}$ (b) from the algebra $\mathcal{R}^{\mathrm{o}}$ to $\mathcal{A}(\mathrm{b})$. The algebra $\mathcal{A}(\mathrm{b})$ contains the sets of plays which can be approximated by closed sets from the inside and open sets from the outside in such a way that the $\psi^{\circ}(\mathrm{b})$ probability of their difference can be made arbitrarily small (see their Proposition 2.1). In Theorem 5.1 they show that all elements of the Borel sigmaalgebra on $\mathrm{P}$ can be approximated this way, so $\sigma(\mathrm{P}) \subseteq \mathcal{A}(\mathrm{b})$. This means that for every behavior strategy $b$, there is a charge defined on $\sigma(\mathrm{P})$ which agrees with $\psi^{\circ}(b)$ on the algebra $\mathcal{R}^{\circ}$. Therefore this charge is consistent with all of our approaches. They have an example with a behavior strategy which does not induce a unique expected payoff even under their approach. 


\subsection{THE MAIN RESULTS}

In this section we present our main results. The proof can be found in Appendix 5.A.

Recall the definition of equivalent strategies from Definition 5.5.4. The next theorem discusses the equivalence between mixed and behavior strategies.

Theorem 5·7.1 (Existence of equivalent strategies).

1. Let $\mathscr{P}$ be an algebra of $\mathrm{P}$, and $\psi^{\mathscr{P}}$ be a $\mathscr{P}$-specification. Then, for every behavior strategy there exists a $\mathscr{P}$-equivalent mixed strategy.

2. There exists a mixed strategy $\mathrm{m}$, in any decision problem ${ }^{3}$, that does not have an $\mathcal{R}^{s+s}$-equivalent behavior strategy. Consequently, $\mathrm{m}$ has no $\mathcal{R}^{\mathrm{o}}$-equivalent behavior strategy either.

In Example 5.7.5 we show a mixed strategy that does not have an $\mathcal{R}^{s+s}$ equivalent behavior strategy. Moreover, every behavior strategy gives a strictly lower expected payoff than this mixed strategy.

We leave it as an open question whether for every mixed strategy $m$ there exists a behavior strategy $b$ that is equivalent to $m$ according to Approach $I$ or Approach II. We sketch the idea for constructing an equivalent behavior strategy $b$ for Approach I. Let a mixed strategy $m$ be given, and let $m^{*}$ be the charge it induces on the set P of plays, as defined in (8). We could find the $\mathcal{R}^{\text {hs }}$-equivalent behavior strategy b by disintegrating $m^{*}$. First we define $\mathrm{b}(\varnothing)$ by for every $\mathrm{D} \subseteq A$ letting $\mathrm{b}(\varnothing)(D)=\mathrm{m}^{*}([\mathrm{D}])$. Let $\mathrm{g}: A \rightarrow \mathcal{C}\left(A, 2^{A}\right)$ be such that for every $E \subseteq A^{2}$

$$
m^{*}([E])=\int_{a_{1} \in A} \int_{a_{2} \in A} \mathbb{I}_{E}\left(a_{1}, a_{2}\right) g\left(a_{1}\right)\left(d a_{2}\right) b(\varnothing)\left(d a_{1}\right) .
$$

If it is possible to disintegrate $m^{*}$, so $g$ exists, then set $b\left(a_{1}\right)=g\left(a_{1}\right)$ for all $a_{1} \in A$. If disintegration is possible, then this can be continued recursively. In that case the behavior strategy $b$ would be $\mathcal{R}^{\text {hs }}$-equivalent to $\mathrm{m}$.

The next theorem provides, for each approach separately, a connection between each behavior strategy inducing a unique expected payoff and properties of the payoff function.

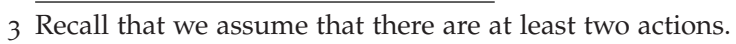


Theorem 5.7.2 (Unique expected payoff under behavior strategies).

Let $\mathrm{u}: \mathrm{P} \rightarrow \mathbb{R}$ be a bounded function.

- Approach I. The following are equivalent:

- Every behavior strategy $\mathrm{b}$ induces a unique expected payoff for $\mathrm{u}$, i.e., $\mathrm{U}^{\mathrm{hs}}(\mathrm{b})$ is a singleton.

- The payoff function $\mathrm{u}$ is uniformly continuous.

- The payoff function $\mathrm{u}$ is uniformly $\mathcal{R}^{\text {hs }}$-approachable.

- Approach II. The following are equivalent:

- Every behavior strategy $\mathrm{b}$ induces a unique expected payoff for $u$, i.e., $\mathrm{U}^{\mathrm{s}}(\mathrm{b})$ is a singleton.

- The payoff function $\mathrm{u}$ is continuous.

- The payoff function $\mathrm{u}$ is uniformly $\mathcal{R}^{\mathrm{s}}$-approachable.

- Approach III:

1. If the payoff function $\mathfrak{u}$ is uniformly $\mathcal{R}^{s+s}$-approachable, then every behavior strategy $\mathrm{b}$ induces a unique expected payoff, i.e., $\mathrm{U}^{\mathrm{s}+\mathrm{s}}(\mathrm{b})$ is a singleton.

2. If the payoff function $\mathfrak{u}$ is strongly tame, then $\mathfrak{u}$ is uniformly $\mathcal{R}^{s+s}$ approachable and hence every behavior strategy $\mathrm{b}$ induces a unique expected payoff.

- Approach IV:

1. If the payoff function $u$ is uniformly $\mathcal{R}^{\mathrm{o}}$-approachable, then every behavior strategy $\mathrm{b}$ induces a unique expected payoff, i.e., $\mathrm{U}^{\mathrm{o}}(\mathrm{b})$ is a singleton.

2. The payoff function $u$ is uniformly $\mathcal{R}^{\mathrm{o}}$-approachable if any of the following sufficient conditions hold:

a) the function $\mathrm{u}$ is weakly tame,

b) the function $\mathrm{u}$ is upper semi-continuous,

c) the function $u$ is lower semi-continuous.

Hence under any of these conditions every behavior strategy $\mathrm{b}$ induces a unique expected payoff. 
Theorem 2.8.5 in Dubins and Savage (2014) resembles the part on Approach II in the foregoing theorem.

The following example shows that under Approach III with stop rules and singletons, every behavior strategy $b$ inducing a unique expected payoff does not imply that the payoff function $u$ is $\mathcal{R}^{s+s}$-approachable or strongly tame. The example shows a payoff function that is neither $\mathcal{R}^{s+s}$-approachable nor strongly tame, however the $\mathrm{U}^{\mathrm{s}+\mathrm{s}}(\mathrm{b})$ is a singleton for every behavior strategy b.

Example 5.7.3. Consider the decision problem with action space $A=$ $\left\{a, a^{\prime}, a^{\prime \prime}\right\}$. Let $W^{a}$ be the set of plays $p$ such that, for some $t \in \mathbb{N}$, the play $p$ contains action $a^{\prime}$ at all periods $1, \ldots, t-1$ and contains action $a$ at period $t$. Similarly, let $W^{a^{\prime \prime}}$ be the set of plays $p$ such that, for some $t \in \mathbb{N}$, the play $p$ contains action $a^{\prime}$ at all periods $1, \ldots, t-1$ and contains action $a^{\prime \prime}$ at period t. Thus $W^{a}, W^{a^{\prime \prime}}$ and the singleton play $\left(a^{\prime}, a^{\prime}, \ldots\right)$ form a partition of $P$.

Consider the following payoff function: let $u(p)=1$ for all $p \in W^{a}$, let $u(p)=-1$ for all $p \in W^{a^{\prime \prime}}$, and let $u\left(a^{\prime}, a^{\prime}, \ldots\right)=0$.

A. Notice that $W^{a} \in \mathcal{R}^{o}$ as $W^{a}$ is an open set. Now we argue that $W^{a} \notin \mathcal{R}^{s+s}$ by contradiction. Assume that $W^{a} \in \mathcal{R}^{s+s}$, then by Lemma 5.6.5

$$
W^{\mathrm{a}}=\left([R] \cup \mathrm{Q}_{1}\right) \backslash \mathrm{Q}_{2}
$$

where $[R] \in \mathcal{R}^{s}$ and $Q_{1}$ and $Q_{2}$ are finite subsets of $P$, and $[R] \cap Q_{1}=\emptyset$, $\mathrm{Q}_{1} \cap \mathrm{Q}_{2}=\emptyset$ and $[\mathrm{R}] \supseteq \mathrm{Q}_{2}$.

There are two distinct possibilities, either the play $p=\left(a^{\prime}, a^{\prime}, \ldots\right) \in[R]$ or $p \notin$ $[R]$. First assume that $p \in[R]$. Since $[R]$ is an open set, there is a history $h \in H$ such that $h \prec p$ and $[h] \subseteq[R]$. Since $[h] \cap W^{a^{\prime \prime}}$ is infinite, the intersection $[R] \cap W^{a^{\prime \prime}}$ is also infinite. The set $Q_{2}$ is finite and $W^{a} \cap W^{a^{\prime \prime}}=\emptyset$, so we reach a contradiction. Now assume that $p \notin[R]$. By $[R] \in \mathcal{R}^{s}$ and part 3 of Lemma 5.6.2 the complement $P \backslash[R]$ is open. Hence there exists a history $h \in H$ such that $h \prec p$ and $[h] \subseteq P \backslash[R]$. The set $Q_{1}$ is finite and $W^{a} \cap[h]$ is infinite, so we reach a contradiction. In conclusion, $W^{\mathrm{a}} \notin \mathcal{R}^{s+s}$.

It is peculiar that if we took an action space consisting only of actions a and $a^{\prime}$, but we defined $W^{a}$ in the same way as above, then $W^{a}=P \backslash\{p\} \in \mathcal{R}^{s+s}$ would hold. In this new decision problem the set of plays is $W^{a} \cup\{p\}$, and 
the restriction of the payoff function $u$ on this set is strongly tame, unlike in the original decision problem.

B. We show that $u$ is not $\mathcal{R}^{s+s}$-approachable. We show that if $\varepsilon=\frac{1}{3}$, then for any $\mathcal{R}^{s+s}$-measurable simple-function $u^{\prime}$ there is a play $p^{\prime} \in \mathrm{P}$ such that $\left|u\left(p^{\prime}\right)-u^{\prime}\left(p^{\prime}\right)\right|>\varepsilon$.

Take an $\mathcal{R}^{s+s}$-measurable simple-function $u^{\prime}=\sum_{i=1}^{m} r_{i} \mathbb{I}_{R_{i}}$ where $m \in \mathbb{N}$, $r_{1}, \ldots, r_{m} \in \mathbb{R}, R_{1}, \ldots, R_{m} \in \mathcal{R}^{s+s}$ and $\left\{R_{1}, \ldots, R_{m}\right\}$ is a partition of $P$. There is an $i \in\{1, \ldots, m\}$ such that $p \in R_{i}$. Assume that $\left|R_{i}\right|>1$, then there is a $p^{\prime} \in\left(R_{i} \cap W^{a}\right) \cup\left(R_{i} \cap W^{a^{\prime \prime}}\right)$. Therefore $\left|u\left(p^{\prime}\right)-r_{i}\right|>\varepsilon$ or $\left|u(p)-r_{i}\right|>\varepsilon$. On the other hand, assume that $\left|R_{i}\right|=1$. Additionally, assume that there is a $j \in\{1, \ldots, m\}$ such that there is a play $p^{\prime} \in R_{j} \cap W^{a}$ and a play $p^{\prime \prime} \in$ $R_{j} \cap W^{a^{\prime \prime}}$. Then $\left|u\left(p^{\prime}\right)-r_{j}\right|>\varepsilon$ or $\left|u\left(p^{\prime \prime}\right)-r_{j}\right|>\varepsilon$. An alternative case would be assuming that there is no such $j$, so for every $j \neq i$ either $R_{j} \subset W^{a}$ or $R_{j} \subset W^{a^{\prime \prime}}$. However there is no finite partition of $P$ in $\mathcal{R}^{s+s}$ that satisfies this condition, since $W^{a} \notin \mathcal{R}^{s+s}$ and $\mathcal{R}^{s+s}$ is an algebra. Since we covered all cases of $\mathcal{R}^{s+s}$-measurable simple-functions, and in each case we found a play $p^{\prime} \in P$ such that $\left|u\left(p^{\prime}\right)-u^{\prime}\left(p^{\prime}\right)\right|>\varepsilon$, we can conclude that $u$ is not $\mathcal{R}^{s+s}$-approachable.

C. We show that $\mathrm{U}^{\mathrm{s}+\mathrm{s}}(\mathrm{b})$ is a singleton for any behavior strategy $\mathrm{b}$. Take a behavior strategy $b$. It is enough to show that $B\left(W^{a}\right)=B^{\prime}\left(W^{a}\right)$ for all $\mathrm{B}, \mathrm{B}^{\prime} \in<\mathrm{b} \mid \mathcal{R}^{s+s}, \psi^{s+s}>$, because $\{\mathrm{p}\} \in \mathcal{R}^{s+s}$ and $\mathrm{W}^{\mathrm{a}} \cup \mathrm{W}^{\mathrm{a}^{\prime \prime}} \cup\{\mathrm{p}\}=\mathrm{P}$. For all $k \in \mathbb{N}$, let $h_{k}^{a} \in A^{k}$ be the history that contains action $a^{\prime}$ at all periods $1, \ldots, k-1$ and contains action $a$ at period $k$. Similarly, for all $k \in \mathbb{N}$, let $h_{k}^{a^{\prime \prime}} \in A^{k}$ be the history that contains action $a^{\prime}$ at all periods $1, \ldots, k-1$ and contains action $a^{\prime \prime}$ at period $k$. For all $t \in \mathbb{N}$, let $S_{t}^{a} \subseteq H$ be the set of histories that consists of $h_{k}^{a}$ for all $k \leqslant t$. Similarly, for all $t \in \mathbb{N}$, let $S_{t}^{a^{\prime \prime}} \subseteq H$ be the set of histories that consists of $h_{k}^{a^{\prime \prime}}$ for all $k \leqslant t$. Finally, for all $t \in \mathbb{N}$, let $\left(a^{\prime}\right)^{t} \in A^{t}$ be the history that contains action $a^{\prime}$ at all periods $1, \ldots, t$.

Take an arbitrary extension $B \in<\mathrm{b} \mid \mathcal{R}^{s+s}, \psi^{s+s}>$. Take a $\mathrm{t} \in \mathbb{N}$. Since $\left[S_{t}^{a}\right],\left[S_{t}^{a^{\prime \prime}}\right]$ and $\left[\left(a^{\prime}\right)^{t}\right]$ form a finite partition of $P$,

$$
\psi^{s+s}(b)\left(\left[S_{t}^{a}\right]\right)+\psi^{s+s}(b)\left(\left[S_{t}^{a^{\prime \prime}}\right]\right)=1-\psi^{s+s}(b)\left(\left[\left(a^{\prime}\right)^{t}\right]\right) .
$$

It follows from $\left[S_{t}^{a}\right] \subset W^{a}$ that $\psi^{s+s}(b)\left(\left[S_{t}^{a}\right]\right) \leqslant B\left(W^{a}\right)$. Note that if $t \leqslant t^{\prime}$, then $\psi^{s+s}(b)\left(\left[S_{t}^{a}\right]\right) \leqslant \psi^{s+s}(b)\left(\left[S_{t^{\prime}}^{a}\right]\right)$. Thus $\lim _{t \rightarrow \infty} \psi^{s+s}(b)\left(\left[S_{t}^{a}\right]\right)$ exists, and 
let us denote it by $l^{a}$. By a similar argument, let $l^{a^{\prime \prime}}=\lim _{t \rightarrow \infty} \psi^{s+s}(b)\left(\left[S_{t}^{a^{\prime \prime}}\right]\right)$. By definition, $\psi^{s+s}(b)(p)=\lim _{t \rightarrow \infty} \psi^{s+s}(b)\left(\left[\left(a^{\prime}\right)^{t}\right]\right)$. Hence

$$
l^{a}+l^{a^{\prime \prime}}=1-\psi^{s+s}(b)(p) .
$$

Since $B$ is a charge on the power set P of plays, $B\left(W^{a}\right)+B\left(W^{a^{\prime \prime}}\right)=1-B(p)$. It follows from $\{p\} \in \mathcal{R}^{s+s}$ that $B(p)=\psi^{s+s}(b)(p)$, so

$$
B\left(W^{a}\right)+B\left(W^{a^{\prime \prime}}\right)=1-\psi^{s+s}(b)(p) .
$$

It follows from $\psi^{s+s}(b)\left(\left[S_{t}^{a}\right]\right) \leqslant B\left(W^{a}\right)$ for all $t \in \mathbb{N}$ that $l^{a} \leqslant B\left(W^{a}\right)$. Similarly, $l^{a^{\prime \prime}} \leqslant B\left(W^{a^{\prime \prime}}\right)$. Based on these last two inequalities, (18) and (19) we can conclude that $l^{a}=B\left(W^{a}\right)$ and $l^{a^{\prime \prime}}=B\left(W^{a^{\prime \prime}}\right)$. Since $l^{a}$ and $l^{a^{\prime \prime}}$ depends only on the behavior strategy $b$, but does not depend on the extension $B$, these equalities hold for any extension. Thus the expected payoff of $b$ is $\mathrm{U}^{\mathrm{s}+\mathrm{s}}(\mathrm{b})=\mathrm{l}^{\mathrm{a}}-\mathrm{l}^{\mathrm{a}^{\prime \prime}}$. Hence, the expected payoff $\mathrm{U}^{\mathrm{s}+\mathrm{s}}(\mathrm{b})$ is a singleton for any behavior strategy $b$, even though the payoff function $u$ is neither $\mathcal{R}^{s+s}$ approachable nor strongly tame.

Comparing the various approaches through examples

REVISITING THE EXAMPLES UNDER APPROACH I First consider Example 5.3.1 again. Let $\tau$ be a pure charge on $\mathbb{N}$, and $b$ be a behavior strategy such that $b(\varnothing)=\tau$ and $b(n)=\delta_{n}$ for every $n \in \mathbb{N}$, where $\delta_{n}$ is the Dirac measure on action $n$. For every $N \in \mathbb{N}$, let $R_{N}=\{(n, n): n \geqslant N\} \subseteq A^{2}$. By iterative integration over the first two periods, for every $N \in \mathbb{N}$ we have $B\left(\left[R_{N}\right]\right)=1$ for every charge $B \in<b \mid \mathcal{R}^{h s}, \psi^{h s}>$, see (9). Hence, $b$ induces a unique expected payoff of 1 , i.e. $\mathrm{U}^{\text {hs }}(\mathrm{b})=1$.

REVISITING THE EXAMPLES UNDER APPROACH II In Example 5.3.1, we found under Approach I that the behavior strategy $b$ induces a unique expected payoff of 1 . Since Approaches I and II are consistent on $\mathcal{R}^{\text {hs }}$ in view of (13), the behavior strategy $b$ induces a unique expected payoff of 1 under Approach II as well.

Now consider Example 5.3.2 and the behavior strategy b described there. For every $t \in \mathbb{N}$, take the stop rule

$$
S_{t}=\{(n, \ldots, n): n \in \mathbb{N} \text { and } n>t\},
$$


where $(n, \ldots, n)$ denotes the history consisting of action $n$ at the first $n$ periods. Since $S_{t}$ is a stop rule, $\left[S_{t}\right] \in \mathcal{S}$ for all $t \in \mathbb{N}$.

Moreover, $\mathrm{P} \backslash\left[\mathrm{S}_{\mathrm{t}}\right]=\left[\mathrm{Q}_{\mathrm{t}}\right]$ where $\mathrm{Q}_{\mathrm{t}}$ is the stop rule $\mathrm{Q}_{\mathrm{t}}=\mathrm{Q}_{1 \mathrm{t}} \cup \mathrm{Q}_{2 \mathrm{t}}$ where

$$
\begin{gathered}
Q_{1 t}=\{(n): n \in \mathbb{N} \text { and } n \leqslant t\} \\
Q_{2 t}=\cup_{n \in \mathbb{N}}\left\{\left(a_{1}, \ldots, a_{n}\right) \in A^{n}: a_{1}=n,\left(a_{1}, \ldots, a_{n}\right) \neq(n, \ldots, n)\right\} .
\end{gathered}
$$

So $P \backslash\left[S_{t}\right] \in \mathcal{S}$. Altogether this means that $\left[S_{t}\right] \in \mathcal{R}^{s}$.

It follows from Lemma 5.6.3 that for every $t \in \mathbb{N}$

$$
\psi^{s}(b)\left(\left[S_{t}\right]\right)=1 .
$$

Consequently, under Approach II, behavior strategy $\mathrm{b}$ induces the unique expected payoff of 1 , i.e. $U^{s}(b)=1$.

We show in the proof of Lemma 5.6.4 that there is an extension $B \in$ $<\mathrm{b} \mid \mathcal{R}^{\text {hs }}, \psi^{\text {hs }}>$ with $B\left([1] \cup\left[S_{1}\right]\right)=0$, so $u(B)=0$. Since $u(B)=0$, but $\mathrm{U}^{\mathrm{s}}(\mathrm{b})=1$, the expected payoff induced by $\mathrm{b}$ is not unique under Approach I.

ReVisiting the examples Under approach iII Since Approach III is consistent with Approach II on the algebra $\mathcal{R}^{s}$ in view of Lemma 5.6.7, the behavior strategies in Examples 5.3.1 and 5.3.2 induce a unique expected payoff of 1 under Approach III as well.

Now consider Examples 5.3.3 and 5.3.4 with the corresponding behavior strategy $b$. Recall that $b(h)(c)=1$ for all histories $h \in H$. It follows from (15) that $\psi^{s+s}(b)(c, c, \ldots)=l^{s+s}(b, p)=1$. In Example 5.3.3 the payoff $u(c, c, \ldots)=1$, so $U^{s+s}(b)=1$. However, in Example 5.3.4 the payoff $u(c, c, \ldots)=0$, so $\mathrm{u}^{\mathrm{s}+\mathrm{s}}(\mathrm{b})=0$.

In part 2 of the proof of Lemma 5.6.7 we show that there is an extension $\mathrm{B} \in<\mathrm{b} \mid \mathcal{R}^{s}, \psi^{s}>$ with $\mathrm{B}(\mathrm{c}, \mathrm{c}, \ldots)=0$. In Example $5.3 .3 \mathrm{u}(\mathrm{p})=0$ for all $p \in P \backslash\{(c, c, \ldots)\}$, so $u(B)=0$ while $U^{s+s}(b)=1$. In Example 5.3.4 $u(p) \geqslant \frac{1}{2}$ for all $p \in P \backslash\{(c, c, \ldots)\}$, so $u(B) \geqslant \frac{1}{2}$ while $U^{s+s}(b)=0$. So in both examples the expected payoff induced by $b$ is not unique under Approach II. Since Approaches I and II are consistent, the expected payoff induced by $b$ is not unique under Approach I either. 
Example 5.7.4 (Another example under Approach III). Consider the decision problem with action space $A=\mathbb{N}$. For every $n \in A$ let $p_{n}$ be the play where action $n$ is played at all periods and let $P_{n}$ be the set of plays that start with action $n$. Let the payoff function $u$ be as follows: for a play $p \in P_{n}$ we set $u(p)=1$ if $p=p_{n}$ and $u(p)=\frac{n}{n+1}$ if $p \in P_{n} \backslash\left\{p_{n}\right\}$. As we have shown in Example $5 \cdot 4 \cdot 5, u$ is strongly tame.

Consider the behavior strategy $b$ which at period 1 plays a pure charge on $A$, and at all later periods repeats the action chosen at period 1. By Theorem $5 \cdot 7 \cdot 2, b$ induces a unique expected payoff of 1 , i.e. $U^{s+s}(b)=1$.

ReVisiting the examples Under approach iv Since Approach IV is consistent with Approach III on the algebra $\mathcal{R}^{s+s}$ in view of Lemma 5.6.10, the behavior strategies in Examples 5.3.1, 5.3.2, 5.3.3 and 5.3.4 induce a unique expected payoff under Approach IV as well.

Now consider Example 5.3.5 with the corresponding behavior strategy b. Recall $b(\varnothing)=\tau$ where $\tau$ is a pure charge on $\mathbb{N}$, and $b(h)=\delta_{1}$ at every other history $h \in H$. For every $n \in \mathbb{N}$, let $p_{n} \in P$ be a play that prescribes action $n$ at the root, and at all other histories it prescribes 1 . For every $t \in \mathbb{N}$, let $V_{t}=$ $\left\{p_{n} \in P: n \in \mathbb{N}, n \geqslant t\right\}$. Let $O_{t}=P \backslash V_{t}$ which is an open set in P. It follows from (17) that $c^{o}(b)\left(O_{t}\right)=\sup _{Z \in \mathcal{R}^{s}, Z \subseteq O_{t}} \psi^{s}(b)(Z)$. Since $\psi^{s}(Z)=0$ for all $\mathrm{Z} \subseteq \mathrm{O}_{\mathrm{t}}, \mathrm{Z} \in \mathcal{R}^{\mathrm{s}}$, the probability $\psi^{\mathrm{o}}(\mathrm{b})\left(\mathrm{O}_{\mathrm{t}}\right)=\mathrm{c}^{\mathrm{o}}(\mathrm{b})\left(\mathrm{O}_{\mathrm{t}}\right)=0$. Because $\left\{\mathrm{O}_{\mathrm{t}}, \mathrm{V}_{\mathrm{t}}\right\}$ is a partition of the set $P$ of plays, $\psi^{o}\left(O_{t}\right)+\psi^{o}(b)\left(V_{t}\right)=\psi^{o}(b)(P)=1$. Thus $\psi^{\mathrm{o}}(\mathrm{b})\left(\mathrm{V}_{\mathrm{t}}\right)=1$, for all $\mathrm{t} \in \mathbb{N}$. This implies that $\mathrm{U}^{\mathrm{o}}(\mathrm{b})=1$.

In part 2 of the proof of Lemma 5.6.10 we show that there is an extension $\mathrm{B} \in<\mathrm{b} \mid \mathcal{R}^{s+s}, \psi^{s+s}>$ with $\mathrm{B}\left(\mathrm{V}_{1}\right)=0$, and so $\mathrm{u}(\mathrm{B})=0$. Since $\mathrm{u}(\mathrm{B})=0$, but $\mathrm{U}^{\mathrm{o}}(\mathrm{b})=1$, the expected payoff induced by $\mathrm{b}$ is not unique under Approach III. Since Approaches I, II and III are consistent, the expected payoff induced by $b$ is not unique under Approaches I and II either.

In the following example we illustrate via Example 5.3.4 that the converse of the statement in part 1 of Theorem 5.7.1 cannot be stated under Approaches III and IV. This counterexample shows that there exists a mixed strategy that does not have an $\mathcal{R}^{s+s}$-equivalent or an $\mathcal{R}^{\circ}$-equivalent behavior strategy, thus it proves part 2 of Theorem 5.7.1. Moreover, we show that every behavior 
strategy induces a strictly lower expected payoff than the expected payoff induced by $m$.

Example 5.7.5. We use the mixed strategy $m$ defined in Example 5.3.4. Let $\mathrm{p}^{*}=(\mathrm{c}, \mathrm{c}, \ldots)$, and let $\mathrm{O}=\mathrm{P} \backslash\left\{\mathrm{p}^{*}\right\}$. Note that $\mathrm{O} \in \mathcal{R}^{\mathrm{s}+\mathrm{s}}$. Take a clopen set $C \subseteq O$. Then $P \backslash C$ is open, and since $p^{*} \in P \backslash C$, there exists a history $h \in H$ such that $h \prec p^{*}$ and $[h] \subseteq P \backslash C$. Based on (8) the probability $m^{*}([h])=1$, so $m^{*}(P \backslash C)=1$. Since $m^{*}$ is finitely additive, $m^{*}(C)=0$. However, $m^{*}(O)=1$.

Assume, by contradiction, that there exists a behavior strategy $b$ that is $\mathcal{R}^{s+s}$ equivalent to $\mathrm{m}$. Then by definition they generate the same charge on the algebra $\mathcal{R}^{s+s}$. Let for every $t \in \mathbb{N}$ the history $h_{t} \in A^{t}$ be $h_{t}=(c, \ldots, c)$. Then $\mathrm{m}^{*}\left(\left[h_{\mathrm{t}}\right]\right)=1$, so $\psi^{\mathrm{s}+\mathrm{s}}(\mathrm{b})\left(\left[h_{\mathrm{t}}\right]\right)=\psi^{\mathrm{s}}(\mathrm{b})\left(\left[h_{\mathrm{t}}\right]\right)=\psi^{\mathrm{hs}}(\mathrm{b})\left(\left[h_{\mathrm{t}}\right]\right)=1$, because $\mathrm{m}$ and $b$ are $\mathcal{R}^{s+s}$-equivalent. Based on (9), for every $t \in \mathbb{N}$ the probability $\mathrm{b}\left(h_{\mathrm{t}}\right)(\mathrm{c})=1$ too. This implies that $\mathrm{l}^{\mathrm{s}+\mathrm{s}}\left(\mathrm{b}, \mathrm{p}^{*}\right)=\prod_{\mathrm{t}=1}^{\infty} \mathrm{b}\left(\mathrm{h}_{\mathrm{t}}\right)(\mathrm{c})=1$. As mentioned earlier, $\mathrm{m}^{*}(\mathrm{O})=1$, thus $\psi^{\mathrm{s}+\mathrm{s}}(\mathrm{b})(\mathrm{O})=\psi^{\mathrm{s}}(\mathrm{b})(\mathrm{P})-\mathrm{l}^{\mathrm{s}+\mathrm{s}}\left(\mathrm{b}, \mathrm{p}^{*}\right)=1$ as well. It follows from $\psi^{s}(b)(P)=1$ that $l^{s+s}\left(b, p^{*}\right)=0$. Since $l^{s+s}\left(b, p^{*}\right)$ cannot be 0 and 1 at the same time, we have reached a contradiction.

Hence there does not exist a behavior strategy $\mathrm{b}$ that is $\mathcal{R}^{s+s}$-equivalent to the mixed strategy m. By part 1 of Lemma 5.6.10 this implies that there does not exist a behavior strategy $b$ that is $\mathcal{R}^{o}$-equivalent to the mixed strategy $m$ either.

Now we focus on the expected payoffs induced by the strategies. As argued in Example 5.3.4, the expected payoff induced by $m$ is 1, i.e. $U(m)=1$. Consider a behavior strategy $b$ such that there exists a history $h$ where $\mathrm{b}(\mathrm{h})(\mathrm{s})>0$. Then $\mathrm{u}^{\mathrm{s}+\mathrm{s}}(\mathrm{b})<1$ and $\mathrm{u}^{\mathrm{o}}(\mathrm{b})<1$. On the other hand, let $\mathrm{b}$ be the behavior strategy with $b(h)(c)=1$ for all histories $h$. According to (15), $\psi^{s+s}(b)(c, c, \ldots)=l^{s+s}(b,(c, c, \ldots))=1$. Since $u(c, c, \ldots)=0$, the expected payoff $\mathrm{U}^{\mathrm{s}+\mathrm{s}}(\mathrm{b})=0$. Due to part $\mathrm{I}$ of Lemma 5.6.10, $\mathrm{U}^{\mathrm{o}}(\mathrm{b})=\mathrm{U}^{\mathrm{s}+\mathrm{s}}(\mathrm{b})=0$ as well. So $\mathrm{U}^{\mathrm{s}+\mathrm{s}}(\mathrm{b})<\mathrm{U}(\mathrm{m})=1$ and $\mathrm{U}^{\mathrm{o}}(\mathrm{b})<\mathrm{U}(\mathrm{m})$ for all behavior strategies $\mathrm{b}$. Thus all behavior strategies induce expected payoffs (under Approaches III and IV) strictly lower than the expected payoff induced by the mixed strategy m. 


\subsection{CONCLUDING REMARKS}

We consider decision problems with infinitely many time periods, and examine finitely additive strategies a decision maker can play. All pure and mixed strategies induce a unique charge on the set of plays, and so they induce a unique expected payoff. However, a behavior strategy may induce multiple charges on the set of plays, possibly even when it prescribes a Dirac measure at every history. So a behavior strategy may induce a set of expected payoffs. We consider four approaches to this problem. We prove consistency amongst these approaches, and illustrate the differences through examples.

With respect to all four approaches, we provide sufficient topological conditions on the payoff function under which each behavior strategy induces a unique expected payoff. We also consider equivalence between mixed and behavior strategies. Each behavior strategy has an equivalent mixed strategy according to all four approaches. However, the converse does not hold under Approaches III and IV, so in every decision problem there is a mixed strategy without an equivalent behavior strategy. We leave it as an open question whether every mixed strategy has an equivalent behavior strategy under Approach I or under Approach II.

AN Alternative to ApProach IV In Approach IV we examined the algebra $\mathcal{R}^{\mathrm{o}}$ generated by the open sets. The starting point was, following Dubins (1974), to define the probability of an open set, under a behavior strategy, through inner approximations by clopen sets. An alternative to this approach would be to take outer approximations by clopen sets instead. We do not go into details whether or not defining the probabilities of open sets this way can be extended to the whole algebra $\mathcal{R}^{\circ}$, just as Dubins (1974) did it with inner approximations. We rather illustrate by the following example that this alternative approach would contradict Approach III (and hence Approach IV as well).

Consider the decision problem in Example 5.3.3 with action space $A=\{c, s\}$, and payoff function $u$ given by $u(p)=1$ and $u\left(p^{\prime}\right)=0$ for all $p^{\prime} \in P \backslash\{p\}$, where $p=(c, c, \ldots)$. Let $b$ denote the behavior strategy that chooses action $c$ with probability 1 at every history. Consider the open set $P \backslash\{p\}$. 
Approach III, under $b$, assigns probability 1 to $\{p\}$ and hence probability 0 to $P \backslash\{p\}$. Now we investigate the probability of the open set $P \backslash\{p\}$ under the alternative approach with outer approximations. Consider any clopen set $Z \supseteq P \backslash\{p\}$. Then by Lemma 5.6.2 the set $Z \in \mathcal{R}^{\text {hs }}$. It follows directly that there is a history $h=(c, \ldots, c)$ such that $[h] \subseteq Z$. Hence the set $Z$ has probability 1 under $b$. This implies that the alternative approach assigns probability 1 to $\mathrm{P} \backslash\{p\}$, in contrast with Approach III. In fact, the alternative approach even violates the viewpoint of countable additivity.

DECISION PROBLEMS With CHANCE MOVES It is an interesting question how to deal with decision problems in which chance moves are present. In such a decision problem it is not clear how to calculate the expected payoff for a pure strategy. Indeed, if we fix a pure strategy for the decision maker, then we can think of chance as a single decision maker who chooses his actions according to a fixed probabilistic rule. As we know from the main body of the chapter, there is an essential difference whether we model the choices of chance by a mixed or by a behavior strategy. This adds an additional dimension to the whole investigation.

MULTIPLE DeCision MAKeRs When there are multiple decision makers the problem becomes substantially more difficult. Suppose that there are two decision makers who use mixed strategies $m_{1}$ and $m_{2}$ respectively. The first question is which charge arises under $\left(m_{1}, m_{2}\right)$ on the set of pure strategy pairs $\left(f_{1}, f_{2}\right)$. The pair $\left(m_{1}, m_{2}\right)$ naturally induces the probability $m_{1}\left(F_{1}^{\prime}\right) \cdot m_{2}\left(F_{2}^{\prime}\right)$ on each rectangle $F_{1}^{\prime} \times F_{2}^{\prime}$ where $F_{1}^{\prime}$ and $F_{2}^{\prime}$ are subsets of the sets $F_{1}$ and $F_{2}$ of pure strategies for the decision makers. The probabilities on rectangles generally do not have a unique extension to a charge on the power set of $F_{1} \times F_{2}$. Consequently, the expected payoff under $\left(m_{1}, m_{2}\right)$ is not always uniquely defined. The problem is quite similar to what we encounter in Chapter 3 .

\section{A THE PROOFS}

Proof of Lemma 5.4.4: Take an arbitrary play $p \in$ P. Consider a sequence of plays $\left(p_{k}\right)_{k \in \mathbb{N}}$ that converges to $p$. We show that $\left(L_{g}\left(p_{k}\right)\right)_{k \in \mathbb{N}}$ converges to $L_{g}(p)$. 
By the definition of $L_{g}$, for every $k \in \mathbb{N}$ there exists a play $q_{k} \in P$ such that $d\left(p_{k}, q_{k}\right)<\frac{1}{k}$ and $\left|L_{g}\left(p_{k}\right)-g\left(q_{k}\right)\right|<\frac{1}{k}$. Since $d\left(p_{k}, q_{k}\right)<\frac{1}{k}$ for all $k \in \mathbb{N}$, the sequence $\left(q_{k}\right)_{k \in \mathbb{N}}$ converges to $p$ as well. Hence by the definition of $L_{g}$, the sequence $\left(g\left(q_{k}\right)\right)_{k \in \mathbb{N}}$ converges to $L_{g}(p)$. By using

$$
\left|L_{g}\left(p_{k}\right)-L_{g}(p)\right| \leqslant\left|L_{g}\left(p_{k}\right)-g\left(q_{k}\right)\right|+\left|g\left(q_{k}\right)-L_{g}(p)\right|,
$$

we obtain that $\left|L_{g}\left(p_{k}\right)-L_{g}(p)\right|$ converges to 0 .

Proof of Lemma 5.5.1: Take an arbitrary mixed strategy $\mathrm{m}$.

Assume first that $u$ is a $\left(2^{\mathrm{P}}\right.$-measurable) simple-function. Then, $u$ is of the form $u(p)=\sum_{i=1}^{n} c_{i} \cdot \mathbb{I}_{Q_{i}}(p)$, where $c_{i} \in \mathbb{R}$ for each $i=1, \ldots, n$, where $\left\{Q_{i}: i=1, \ldots, n\right\}$ is a partition of P. For each $i=1, \ldots, n$, let $F_{i}=\{f \in F$ : $\left.p_{f} \in Q_{i}\right\}$. Thus, $\left\{F_{i}: i=1, \ldots, n\right\}$ is a partition of the set $F$ of pure strategies, $U(f)=\sum_{i=1}^{n} c_{i} \cdot \mathbb{I}_{F_{i}}(f)$ for all $f \in F$, and $m^{*}\left(Q_{i}\right)=m\left(F_{i}\right)$ for each $i=1, \ldots, n$ by (8).

Hence, we have

$$
\begin{aligned}
\int_{p \in P} u(p) m^{*}(d p) & =\sum_{i=1}^{n} c_{i} \cdot \int_{p \in P} \mathbb{I}_{Q_{i}}(p) m^{*}(d p)=\sum_{i=1}^{n} c_{i} \cdot m^{*}\left(Q_{i}\right) \\
& =\sum_{i=1}^{n} c_{i} \cdot m\left(F_{i}\right)=\sum_{i=1}^{n} c_{i} \cdot \int_{f \in F} \mathbb{I}_{F_{i}}(f) m(d f) \\
& =\int_{f \in F} U(f) m(d f)=U(m) .
\end{aligned}
$$

Now let $u$ be an arbitrary bounded payoff function. Let $\varepsilon>0$ and let $u^{\prime}$ : $\mathrm{P} \rightarrow \mathbb{R}$ be a simple-function such that $\left|\mathfrak{u}(p)-\mathfrak{u}^{\prime}(p)\right| \leqslant \varepsilon$ for every play $p \in P$. Hence, also $\left|U(f)-U^{\prime}(f)\right| \leqslant \varepsilon$ for every pure strategy $f \in F$. We have

$$
\begin{aligned}
U(m) & =\int_{f \in F} u(f) m(d f) \leqslant \int_{f \in F} u^{\prime}(f) m(d f)+\varepsilon \\
& =\int_{p \in P} u^{\prime}(p) m^{*}(d p)+\varepsilon \leqslant \int_{p \in P} u(p) m^{*}(d p)+2 \varepsilon,
\end{aligned}
$$

and similarly $U(m) \geqslant \int_{p \in P} u(p) m^{*}(d p)-2 \varepsilon$. Hence

$$
\left|\mathrm{U}(\mathrm{m})-\int_{\mathrm{p} \in \mathrm{P}} \mathrm{u}(\mathrm{p}) \mathrm{m}^{*}(\mathrm{dp})\right| \leqslant 2 \varepsilon .
$$

Since $\varepsilon>0$ was arbitrary, the proof is complete. 
Proof of Lemma 5.5.2: The empty set $\emptyset$ belongs to $\mathcal{R}^{\text {hs }}$, as it is induced by the empty stop rule: $[\emptyset]=\emptyset$.

Now we show that $\mathcal{R}^{\text {hs }}$ is closed under taking complements. Let $[R] \in \mathcal{R}^{\text {hs }}$, where $R \subseteq A^{n}$ for some $n \in \mathbb{N}$. Then, $P \backslash[R]=\left[R^{\prime}\right]$, where $R^{\prime}=A^{n} \backslash R$. Hence, $\mathrm{P} \backslash[\mathrm{R}] \in \mathcal{R}^{\text {hs }}$.

Finally, we show that $\mathcal{R}^{\text {hs }}$ is closed under taking unions. Let $[R] \in \mathcal{R}^{\text {hs }}$ and $\left[R^{\prime}\right] \in \mathcal{R}^{\text {hs }}$, where $R \subseteq A^{n}$ for some $n \in \mathbb{N}$ and $R^{\prime} \subseteq A^{n^{\prime}}$ for some $n^{\prime} \in \mathbb{N}$. Let $\mathrm{N}=\max \left\{\mathrm{n}, \mathrm{n}^{\prime}\right\}$. Let

$$
\begin{gathered}
\mathrm{Q}=\left\{h \in A^{N} \text { : there exists } g \in R \text { with } g \preceq h\right\} \\
Q^{\prime}=\left\{h \in A^{N} \text { : there exists } g \in R^{\prime} \text { with } g \preceq h\right\} .
\end{gathered}
$$

Clearly, $[R]=[Q]$ and $\left[\mathrm{R}^{\prime}\right]=\left[\mathrm{Q}^{\prime}\right]$. Hence

$$
[R] \cup\left[R^{\prime}\right]=[Q] \cup\left[Q^{\prime}\right]=\left[Q \cup Q^{\prime}\right] .
$$

As $\mathrm{Q} \cup \mathrm{Q}^{\prime} \subseteq \mathrm{A}^{\mathrm{N}}$, we conclude $[R] \cup\left[\mathrm{R}^{\prime}\right] \in \mathcal{R}^{\mathrm{hs}}$.

\section{Proof of Theorem 5.5.5:}

Let $\mathscr{P}$ denote an algebra of $\mathrm{P}$, and $\psi^{\mathscr{P}}$ be a $\mathscr{P}$-specification, and suppose that the payoff function $u$ is uniformly $\mathscr{P}$-approachable. Consider a behavior strategy $b$.

Let $\varepsilon>0$. Since $u$ is uniformly $\mathscr{P}$-approachable, there exist $c_{i} \in \mathbb{R}$ and $\mathrm{Q}_{i} \in \mathscr{P}$ for each $i=1, \ldots, n$, and where $\left\{Q_{i}: i=1, \ldots, n\right\}$ is a partition of $P$ such that for all $p \in P$

$$
\sum_{i=1}^{n}\left(c_{i}-\varepsilon\right) \cdot \mathbb{I}_{Q_{i}}(p) \leqslant u(p) \leqslant \sum_{i=1}^{n}\left(c_{i}+\varepsilon\right) \cdot \mathbb{I}_{Q_{i}}(p) .
$$

Let $B \in<b \mid \mathscr{P}, \psi^{\mathscr{P}}>$. As $Q_{i} \in \mathscr{P}$ we have $B\left(Q_{i}\right)=\psi^{\mathscr{P}}(b)\left(Q_{i}\right)$, for every $i=1, \ldots, n$. By monotonicity of the integral, by the definition of integration of a $\mathscr{P}$-measurable simple-function and by (10)

$$
\sum_{i=1}^{n}\left(c_{i}-\varepsilon\right) \cdot \psi^{\mathscr{P}}(b)\left(Q_{i}\right) \leqslant u(B) \leqslant \sum_{i=1}^{n}\left(c_{i}+\varepsilon\right) \cdot \psi^{\mathscr{P}}(b)\left(Q_{i}\right) .
$$

This inequality holds for $u\left(B^{\prime}\right)$ for any $B^{\prime} \in<b \mid \mathscr{P}, \psi^{\mathscr{P}}>$ as well, hence

$$
-2 \varepsilon \leqslant u(B)-u\left(B^{\prime}\right) \leqslant 2 \varepsilon .
$$


Since this can be derived for all $\varepsilon>0$, it follows that $\mathfrak{u}(B)=\mathfrak{u}\left(\mathrm{B}^{\prime}\right)$. So $\mathrm{U}\left(\mathrm{b} \mid \mathscr{P}, \psi^{\mathscr{P}}\right)$ is a singleton, as claimed.

\section{Proof of Lemma 5.6.1:}

Proof of part 1. Take a set $[R] \in \mathcal{R}^{\text {hs }}$. Because $[h]$ is open for all histories $h$ and $[R]=\cup_{h \in R}[h]$, the set $[R]$ is open. Since by Lemma 5.5.2 the complement of $[R]$ is also in $\mathcal{R}^{\text {hs }}$, the set $[R]$ is closed as well. Hence, $[R]$ is clopen.

\section{Proof of part 2.}

$\Longrightarrow$ Assume first that the action set $A$ is infinite. We show that there exists a set $U \in C L \backslash \mathcal{R}^{\text {hs }}$. (The construction is related to Example 5.3.2.) Since $A$ is infinite, by renaming the actions we can assume that $\mathbb{N} \subseteq A$. For every $n \in \mathbb{N}$, let $h_{n}$ denote the history $(n, \ldots, n)$ of length $n$. Define

$$
\mathrm{U}=\bigcup_{n \in \mathbb{N}}\left[h_{n}\right] .
$$

Thus, $\mathrm{U}$ is the set of plays that start with action $n$ at the first $n$ periods, for some $n \in \mathbb{N}$.

We show $\mathrm{U} \in \mathrm{CL} \backslash \mathcal{R}^{\text {hs }}$. It is clear that $\mathrm{U} \notin \mathcal{R}^{\text {hs }}$. So it remains to verify that $U \in C L$. Since $\left[h_{n}\right]$ is open for every $n \in \mathbb{N}$, the set $U$ is open.

For every $n \in \mathbb{N}$, let $H_{n}$ be the set of histories of length $n$ that start with action $n$. Since $\left[H_{n} \backslash\left\{h_{n}\right\}\right]$ is open for every $n \in \mathbb{N}$ and $[A \backslash \mathbb{N}]$ is also open, the set

$$
\mathrm{P} \backslash \mathrm{U}=\cup_{\mathrm{n} \in \mathbb{N}}\left[\mathrm{H}_{\mathrm{n}} \backslash\left\{\mathrm{h}_{\mathrm{n}}\right\}\right] \cup[A \backslash \mathbb{N}]
$$

is open too. Hence, $\mathrm{U}$ is closed, and therefore $\mathrm{U} \in \mathrm{CL}$ indeed.

$\Longleftarrow$ Now assume first that the action set $A$ is finite. For proving $\mathcal{R}^{\text {hs }}=\mathrm{CL}$, in view of part 1 we only need to prove $\mathcal{R}^{\text {hs }} \supseteq$ CL. Let $Z \in C L$. To show $Z \in \mathcal{R}^{\text {hs }}$, it is sufficient to prove that there is $n \in \mathbb{N}$ such that for every history $h \in A^{n}$ we have either $[h] \subseteq Z$ or $[h] \subseteq P \backslash Z$. Indeed, then $Z=[R]$ where $R=\left\{h \in A^{n}:[h] \subseteq Z\right\}$.

Suppose the opposite. Then there exists a sequence of histories $h_{n}$ such that for every $n \in \mathbb{N}$ we have $h_{n} \in A^{n}$ and there exists a play $p_{n} \succ h_{n}$ and a play $q_{n} \succ h_{n}$ with $p_{n} \in Z$ and $q_{n} \in P \backslash Z$. Because $A$ is finite, $P$ is compact. Hence, there exists a subsequence $n_{k}$, with index $k \in \mathbb{N}$, converging to infinity, such that $p_{n_{k}}$ converges to some play $p$. Then there also exists a sub-subsequence 
$n_{k_{m}}$ such that $q_{n_{k m}}$ also converges. Since $p_{n_{k_{m}}} \succ h_{n_{k_{m}}}$ and $q_{n_{k m}} \succ h_{n_{k m}}$ for every $m \in \mathbb{N}$, and the sequence $p_{n_{k_{m}}}$ converges to $p$, we can conclude that $q_{n_{k_{m}}}$ also converges to $p$. However, because $Z$ is clopen, $p \in Z$ as it is the limit of $p_{n_{k m}}$ and $p \in P \backslash Z$ as it is the limit of $q_{n_{k m}}$. This is a contradiction.

Proof of Lemma 5.6.2: First we prove part 1. Since for every stop rule $S \subseteq H$

$$
[S]=\bigcup_{h \in S}[h]
$$

and each $[h]$ is open, the set $[S]$ is open too. Hence, $\mathcal{S}$ only contains open sets of $P$.

Now suppose that $\mathrm{Q} \subseteq \mathrm{P}$ is open. Because $\mathrm{Q}$ is open, for every play $\mathrm{p} \in \mathrm{Q}$ there is a history $h_{p}$ such that $h_{p} \prec p$ and $\left[h_{p}\right] \subseteq Q$. In addition, we assume that $h_{p}$ is the shortest such history. It can be verified that the set $S=\left\{h_{p} \in\right.$ $\mathrm{H}: \mathrm{p} \in \mathrm{Q}\}$ is a stop rule, and $\mathrm{Q}=[\mathrm{S}]$. Thus, $\mathrm{Q} \in \mathcal{S}$.

Part 3 is a consequence of part 1 and the definition of $\mathcal{R}^{s}$. Part 2 then follows, as the set of clopen sets in a topological space is an algebra (and one can also prove it directly from the definition). Part 4 directly follows from the definitions of $\mathcal{R}^{\text {hs }}$ and $\mathcal{R}^{\text {s. Part }} 5$ is due to part 2 of Lemma 5.6.1 and part 3 of Lemma 5.6.2.

\section{Proof of Lemma 5.6.3:}

Let $\Psi$ be the set of functions $\psi$ that to each behavior strategy $\mathrm{b}$ assigns a charge $\psi(b)$ on $\mathcal{R}^{s}$. In part A we prove that there is a unique element $\psi \in \Psi$ with property (12). Then in part B we show that this $\psi$ is an $\mathcal{R}^{s}$-specification.

Part A: Take an arbitrary behavior strategy $\mathrm{b}$ and a set $\mathrm{Q} \in \mathcal{R}^{\mathrm{s}}$. Our goal is to show that the probability $\psi(b)(Q)$ is the same for each $\psi \in \Psi$ that satisfies property (12). This will imply that there is a unique element $\psi \in \Psi$ with property (12). We will use in the proof that $\psi(b)(Q)$ is the same as $\psi\left(\left.b\right|_{\varnothing}\right)\left(\left.\mathrm{Q}\right|_{\varnothing}\right)$.

As $Q \in \mathcal{R}^{s}$, there exist stop rules $S_{1}, S_{2}$ such that $\left[S_{1}\right]=Q$ and $\left[S_{2}\right]=$ $P \backslash Q$. Take a $h \in S_{1}$. Since $Q=\left[S_{1}\right]$, we know $\left.Q\right|_{h}=\left.[h]\right|_{h}=A^{\mathbb{N}}$. Therefore $\psi\left(\left.b\right|_{h}\right)\left(\left.Q\right|_{h}\right)=1$. Similarly, if $h \in S_{2}$, then $\psi\left(\left.b\right|_{h}\right)\left(\left.Q\right|_{h}\right)=\psi(b)(\emptyset)=0$. 
Let $\mathrm{H}^{\mathrm{Q}} \subset \mathrm{H}$ be the set of all histories $\mathrm{h}$ such that there is no $\mathrm{h}^{\prime} \prec \mathrm{h}$ with $\mathrm{h}^{\prime} \in$ $S_{1} \cup S_{2}$. Intuitively, these are the histories that do not come after stopping by either $S_{1}$ or $S_{2}$. Let $H_{0}^{Q}=S_{1} \cup S_{2}$.

Let $\phi_{0}: \mathrm{H}_{0}^{\mathrm{Q}} \rightarrow \mathbb{R}$, and $\phi_{0}(\mathrm{~h})=1$ if $\mathrm{h} \in \mathrm{S}_{1}$ and $\phi_{0}(\mathrm{~h})=0$ if $\mathrm{h} \in \mathrm{S}_{2}$. So $\phi_{0}$ coincides with $\psi\left(\left.b\right|_{h}\right)\left(\left.Q\right|_{h}\right)$ on $h \in H_{0}^{Q}$. Let $z$ be the set of maps $\phi: D_{\phi} \rightarrow \mathbb{R}$, where $\mathrm{H}_{0}^{\mathrm{Q}} \subseteq \mathrm{D}_{\phi} \subseteq \mathrm{H}^{\mathrm{Q}}$ and if $\mathrm{h} \in \mathrm{D}_{\phi} \backslash \mathrm{H}_{0}^{\mathrm{Q}}$, then $(\mathrm{h}, \mathrm{a}) \in \mathrm{D}_{\phi}$ as well, for all $a \in A$. Moreover, $\phi(h)=\phi_{0}(h)$ for all $h \in H_{0}^{Q}$ and based on (12) for every history $h \in \mathrm{D}_{\phi} \backslash \mathrm{H}_{0}^{\mathrm{Q}}$

$$
\phi(h)=\int_{a \in A} \phi(h a) b(h)(d a) .
$$

Take a partial ordering $\triangleleft$ on the set $z$, by $\left(\mathrm{D}_{\phi_{1}}, \phi_{1}\right) \triangleleft\left(\mathrm{D}_{\phi_{2}}, \phi_{2}\right)$ if $\mathrm{D}_{\phi_{1}} \subseteq \mathrm{D}_{\phi_{2}}$ and $\phi_{1}=\phi_{2}$ on $D_{\phi_{1}}$. Take a chain $\mathcal{C} \subseteq$ z. Let $\bar{\phi}$ be $\bar{\phi}(\mathrm{h})=\phi(\mathrm{h})$ whenever $h \in \mathrm{D}_{\phi}$ for a $\phi \in \mathcal{C}$, so $\bar{\phi}$ is defined on $\mathrm{D}_{\bar{\phi}}=\cup_{\phi \in \mathcal{C}} \mathrm{D}_{\phi}$. It follows from $\left(D_{\phi_{0}}, \phi_{0}\right) \triangleleft\left(D_{\bar{\phi}}, \bar{\phi}\right)$ that $\left(D_{\bar{\phi}}, \bar{\phi}\right) \in z$. Hence $\left(D_{\bar{\phi}}, \bar{\phi}\right)$ is an upper bound for the chain $\mathcal{C}$. So every chain in $z$ has an upper bound. Based on the Lemma of Zorn, Z has a maximal element $\left(\mathrm{D}_{\xi}, \xi\right)$.

We show that $\mathrm{D}_{\xi}=\mathrm{H}^{\mathrm{Q}}$. Assume by contradiction that $\mathrm{H}^{\mathrm{Q}} \backslash \mathrm{D}_{\xi}$ is not empty. Also assume that there exists a history $h \in \mathrm{H}^{\mathrm{Q}} \backslash \mathrm{D}_{\xi}$ such that $(\mathrm{h}, \mathrm{a}) \in \mathrm{D}_{\xi}$ for all $a \in A$. Then $\xi(h)$ could be defined through (20) on $D_{\xi} \cup\{h\}$. In that case $\left(D_{\xi}, \xi\right)$ would not be maximal, which is a contradiction. Assume, on the other hand, that for every history $h \in H^{Q} \backslash D_{\xi}$ there exists an action $a \in A$ with $(h, a) \notin D_{\xi}$. Then there exists a history $h \in H^{Q} \backslash D_{\xi}$ and a sequence of actions $\left(a_{t}\right)_{t \in \mathbb{N}}$ where $\left(h, a_{1}, \ldots, a_{t}\right) \notin D_{\xi}$ for all $t \in \mathbb{N}$. Since $H_{0}^{Q} \subseteq D_{\xi}$, we know that $\left(h, a_{1}, \ldots, a_{t}\right) \notin H_{0}^{Q}$ for all $t \in \mathbb{N}$ either. However, this is a contradiction, because $\mathrm{H}_{0}^{\mathrm{Q}}=\mathrm{S}_{1} \cup \mathrm{S}_{2}$ is a complete stop rule.

Finally, we show that $\xi$ is unique. Take a maximal element $\left(D_{\xi^{\prime}}, \xi^{\prime}\right)$ in the set $z$, so $\mathrm{D}_{\xi^{\prime}}=\mathrm{H}^{\mathrm{Q}}$. Assume by contradiction that $\xi$ and $\xi^{\prime}$ are not the same. Since $\left(D_{\xi}, \xi\right),\left(D_{\xi^{\prime}}, \xi^{\prime}\right) \in \mathcal{Z}$, the maps $\xi$ and $\xi^{\prime}$ coincide on the set $H_{0}^{Q}$. Let $\mathrm{N}=\left\{\mathrm{h} \in \mathrm{H}^{\mathrm{Q}} \backslash \mathrm{H}_{0}^{\mathrm{Q}}: \xi^{\prime}(\mathrm{h}) \neq \xi(\mathrm{h})\right\}$. By our assumption, the set $\mathrm{N}$ is not empty. We consider two cases, and in both cases we derive a contradiction. First, assume that there exists a history $h \in N$ such that $(h, a) \in H^{Q} \backslash N$ for all $a \in A$. Since in (20) the charge $b(h)$ is the same irrespective of the map $\phi$, the values $\xi(h)$ and $\xi^{\prime}(h)$ coincide. That means that $h \notin N$, which is a contradiction. On the other hand, assume that there is a history $h \in N$ and 
a sequence of actions $\left(a_{t}\right)_{t \in \mathbb{N}}$ such that $\left(h, a_{1}, \ldots, a_{t}\right) \in N$ as well, for all $t \in \mathbb{N}$. Let $p=\left(h, a_{1}, a_{2}, \ldots\right)$. Because $H_{0}^{Q}=S_{1} \cup S_{2}$ is a complete stop rule, there exists a history $h^{\prime} \in H_{0}^{Q}$ with $h^{\prime} \prec p$. It follows from $h^{\prime} \in H_{0}^{Q}$ and $\left(D_{\xi}, \xi\right),\left(D_{\xi^{\prime}}, \xi^{\prime}\right) \in z$ that $\xi\left(h^{\prime}\right)=\phi_{0}\left(h^{\prime}\right)$ and $\xi^{\prime}\left(h^{\prime}\right)=\phi_{0}\left(h^{\prime}\right)$, so $\xi\left(h^{\prime}\right)=$ $\xi^{\prime}\left(h^{\prime}\right)$. Because $h^{\prime} \prec p$, the history $h^{\prime} \in N$ and so $\xi^{\prime}\left(h^{\prime}\right) \neq \xi\left(h^{\prime}\right)$. This is a contradiction as well. Therefore the set $N$ is empty, and $\left(D_{\xi}, \xi\right)$ is the unique maximal element of $z$.

Since $D_{\xi}=H^{Q}$, the map $\xi$ is also defined at the root. Let $\psi\left(\left.b\right|_{\varnothing}\right)\left(\left.Q\right|_{\varnothing}\right)=\xi(\varnothing)$.

Part B: Now prove that $\psi(b)$ is indeed an $\mathcal{R}^{s}$-specification. Recall that $c^{\text {hs }}(b)([S])=\psi^{\text {hs }}(b)([S])$, by the definition of $\psi^{\text {hs }}$, for every behavior strategy $b$ and for every set $[S] \in \mathcal{R}^{\text {hs }}$. Thus, we need to show that

$$
\psi(b)([S])=\psi^{\text {hs }}(b)([S])
$$

for every behavior strategy $b$ and for every set $[S] \in \mathcal{R}^{\text {hs }}$.

Let for any set $S \subseteq A^{n+1}$, where $n \in \mathbb{N}$, and for any $a \in A$

$$
\left.S\right|_{a}=\left\{\left(r_{2}, r_{3}, \ldots, r_{n+1}\right):\left(a, r_{2}, r_{3}, \ldots, r_{n+1}\right) \in S\right\} .
$$

Hence $\left.S\right|_{a} \subseteq A^{n}$. The function $\psi^{\text {hs }}$ assigns to the induced strategy $\left.b\right|_{a}$ a charge $\psi^{\text {hs }}\left(\left.b\right|_{a}\right)$ on the algebra $\mathcal{R}^{\text {hs }}$.

For every $n \in \mathbb{N}$, let $\Lambda(n)$ be the statement that $\psi^{\text {hs }}(b)([S])=\psi(b)([S])$ for every behavior strategy $\mathrm{b}$ and and every $[S] \in \mathcal{R}^{\text {hs }}$, where $S \subseteq A^{n}$ (for $n=0, A^{0}$ consists of the root, i.e. $A^{0}=\{\varnothing\}$ ). We prove this statement $\Lambda(n)$ by induction on $n$.

If $n=0$, then $S=\emptyset$ or $S=\{\varnothing\}$, so $[S]=\emptyset$ or $[S]=P$. Recall that $\psi^{\text {hs }}(b)$ is a charge on the algebra $\mathcal{R}^{\text {hs }}$ and $\psi(b)$ is a charge on the algebra $\mathcal{R}^{\text {s }}$, and both $\emptyset$ and P belong to these algebras. In the former case $\psi^{\mathrm{hs}}(\mathrm{b})(\emptyset)=\psi(\mathrm{b})(\emptyset)=0$, and in the latter case $\psi^{\text {hs }}(b)(P)=\psi(b)(P)=1$. Hence, $\Lambda(0)$ is true. 
Now assume that $\Lambda(n)$ is true for some $n \in \mathbb{N}$. We prove $\Lambda(n+1)$. Take a behavior strategy $\mathrm{b}$ and $[S] \in \mathcal{R}^{\text {hs }}$, where $S \subseteq A^{\mathrm{n}+1}$. By part $\mathrm{A}$ of the proof of Lemma 5.6.3, we have

$$
\begin{aligned}
\psi(b)([S]) & =\int_{a \in A} \psi\left(\left.b\right|_{a}\right)\left(\left.[S]\right|_{a}\right) b(\varnothing)(d a) \\
& =\int_{a \in A} \psi\left(\left.b\right|_{a}\right)\left(\left[\left.S\right|_{a}\right]\right) b(\varnothing)(d a) \\
& =\int_{a \in A} \psi^{h s}\left(\left.b\right|_{a}\right)\left(\left[\left.S\right|_{a}\right]\right) b(\varnothing)(d a) \\
& =\psi^{h s}(b)([S]) .
\end{aligned}
$$

The first equality follows from (12). For the second equality we use that $\left.[S]\right|_{a}=\left[\left.S\right|_{a}\right]$. In the third equality we use the inclusion $\left.S\right|_{a} \subseteq A^{n}$ for each $a \in A$ and that $\Lambda(n)$ is true by assumption (specifically, in the subproblem $\left.\mathrm{G}\right|_{\mathrm{a}}$ ). The last equality follows from $\psi^{\text {hs }}=\mathrm{c}^{\text {hs }}$, where the latter is defined in (9).

In conclusion, $\Lambda(n)$ is true for all $n \in \mathbb{N}$.

Proof of Lemma 5.6.4: Since $A$ is infinite, by renaming the actions we can assume that $\mathbb{N} \subseteq A$. The proof is making use of the idea of Example 5.3.2.

Consider the following behavior strategy $b$. The charge $b(\varnothing)$ is such that $\mathrm{b}(\varnothing)(\{n\})=0$ for every $\mathrm{n} \in \mathbb{N}$ and $\mathrm{b}(\varnothing)(\mathbb{N})=1$ (essentially, $\mathrm{b}(\varnothing)$ is a pure charge on $\mathbb{N}$ ), and for any other $h=\left(a_{1}, \ldots, a_{t}\right) \in H$ the charge $b(h)$ is simply the Dirac measure on action $a_{1}$. Consider also the set $Z=$ $\cup_{n \in \mathbb{N}}[(n, \ldots, n)]$, where $[(n, \ldots, n)]$ denotes the set of plays that start with action $n$ at the first $n$ periods.

Now we prove that there is a charge $\mu$ on $\mathcal{R}^{s}$ such that for every $[R] \in \mathcal{R}^{\text {hs }}$

$$
\psi^{s}(b)([R])=\mu([R]) \quad \text { and } \quad \psi^{s}(b)(Z) \neq \mu(Z) .
$$

Take an $N \subset \mathbb{N}$, and note that $\psi^{\text {hs }}(b)\left(\cup_{n \in N}[(n, \ldots, n)]\right)=0$. So the inner approximation of $Z$ by sets in $\mathcal{R}^{\text {hs }}$ yields

$$
\sup _{[R] \in \mathcal{R}^{h s},[R] \subseteq Z} \psi^{\text {hs }}(b)([R])=0 .
$$

Based on Theorem 3.C.3 there exists an extension $\mu \in<\mathrm{b} \mid \mathcal{R}^{\text {hs }}, \psi^{\text {hs }}>$ such that $\mu(Z)=0$. Since $\psi^{s}(b)(Z)=1 \neq 0=\mu(Z)$, this is exactly what we were looking for. 


\section{Proof of Lemma 5.6.5:}

Part 1: We show that $\mathcal{R}^{s+s} \supsetneq \mathcal{R}^{s}$, by proving that $\{p\} \in \mathcal{R}^{s+s} \backslash \mathcal{R}^{s}$. Take a play $p \in P$. By definition, $\{p\} \in \mathcal{R}^{s+s}$. Since we assumed that the action space $A$ has at least two actions, the singleton set $\{p\}$ is not open. By part 3 of Lemma 5.6.2, the algebra $\mathcal{R}^{s}$ only contains clopen sets. Thus $\{p\} \notin \mathcal{R}^{s}$.

Part 2: Let $\mathscr{P}$ be the set of all subsets of P of the form (14) given in the lemma. We show that $\mathcal{R}^{s+s}=\mathscr{P}$.

By the definition of $\mathcal{R}^{s+s}$, we have $\mathscr{P} \subseteq \mathcal{R}^{s+s}$. So it remains to prove that $\mathcal{R}^{s+s} \subseteq \mathscr{P}$.

It is clear that (1) $\mathcal{R}^{s} \subseteq \mathscr{P}$ and (2) $\{\mathrm{p}\} \in \mathscr{P}$ for every $\mathrm{p} \in \mathrm{P}$. We show that $\mathscr{P}$ is an algebra. Then, by the definition of $\mathcal{R}^{s+s}$, it will follow that $\mathcal{R}^{s+s} \subseteq \mathscr{P}$.

Obviously, $\mathrm{P} \in \mathscr{P}$, by choosing $\mathrm{R}=\varnothing$ and $\mathrm{Q}_{1}=\mathrm{Q}_{2}=\emptyset$.

Now we show that $\mathscr{P}$ is closed under taking complements. Let $Z \in \mathscr{P}$. Then $Z$ is of the form

$$
\mathrm{Z}=\left([\mathrm{R}] \cup \mathrm{Q}_{1}\right) \backslash \mathrm{Q}_{2}
$$

where $[R] \in \mathcal{R}^{s}$ and $\mathrm{Q}_{1}$ and $\mathrm{Q}_{2}$ are finite subsets of $\mathrm{P}$. The complement of $\mathrm{Z}$ is then

$$
\mathrm{P} \backslash \mathrm{Z}=\left((\mathrm{P} \backslash[\mathrm{R}]) \cup \mathrm{Q}_{2}\right) \backslash\left(\mathrm{Q}_{1} \backslash \mathrm{Q}_{2}\right) .
$$

Because $[\mathrm{R}] \in \mathcal{R}^{s}$, we have $(\mathrm{P} \backslash[\mathrm{R}]) \in \mathcal{R}^{s}$ as well. Hence, $(\mathrm{P} \backslash \mathrm{Z}) \in \mathscr{P}$.

Finally, we show that $\mathscr{P}$ is closed under taking unions. Let $Z, Z^{\prime} \in \mathscr{P}$. Then $Z$ and $Z^{\prime}$ are of the form

$$
\mathrm{Z}=\mathrm{W} \backslash \mathrm{V} \quad \text { and } \quad \mathrm{Z}^{\prime}=\mathrm{W}^{\prime} \backslash \mathrm{V}^{\prime}
$$

where $W=[R] \cup Q$ and $W^{\prime}=\left[R^{\prime}\right] \cup Q^{\prime}$, with $[R],\left[R^{\prime}\right] \in \mathcal{R}^{s}$ and $Q, Q^{\prime}, V$ and $V^{\prime}$ being finite subsets of $P$. For the union of $Z$ and $Z^{\prime}$ we have

$$
Z \cup Z^{\prime}=\left(W \cup W^{\prime}\right) \backslash\left(\left(\left(W \backslash W^{\prime}\right) \cap V\right) \cup\left(\left(W^{\prime} \backslash W\right) \cap V^{\prime}\right)\right) .
$$

Note that $W \cup W^{\prime}=\left([R] \cup\left[R^{\prime}\right]\right) \cup\left(Q \cup Q^{\prime}\right)$. Moreover, $\left([R] \cup\left[R^{\prime}\right]\right) \in \mathcal{R}^{s}$ and $\mathrm{Q} \cup \mathrm{Q}^{\prime}$ is finite. So $\left(Z \cup Z^{\prime}\right) \in \mathscr{P}$.

Part 3: We prove that for every $Z \in \mathcal{R}^{s+s}$ there is a unique representation of the form (14) such that $[R] \cap Q_{1}=\emptyset, Q_{1} \cap Q_{2}=\emptyset$ and $[R] \supseteq Q_{2}$. 
By part A, it is easy to conclude that such a representation exists. Now assume that for some $Z \in \mathcal{R}^{s+s}$ there are two representations

$$
\mathrm{Z}=\left([\mathrm{R}] \cup \mathrm{Q}_{1}\right) \backslash \mathrm{Q}_{2} \quad \text { and } \quad \mathrm{Z}=\left(\left[\mathrm{R}^{\prime}\right] \cup \mathrm{Q}_{1}^{\prime}\right) \backslash \mathrm{Q}_{2}^{\prime}
$$

such that $[R] \cap \mathrm{Q}_{1}=\emptyset, \mathrm{Q}_{1} \cap \mathrm{Q}_{2}=\emptyset$ and $[\mathrm{R}] \supseteq \mathrm{Q}_{2}$, and $\left[\mathrm{R}^{\prime}\right] \cap \mathrm{Q}_{1}^{\prime}=\emptyset, \mathrm{Q}_{1}^{\prime} \cap$ $\mathrm{Q}_{2}^{\prime}=\emptyset$ and $\left[\mathrm{R}^{\prime}\right] \supseteq \mathrm{Q}_{2}^{\prime}$.

We first show that $[R]=\left[R^{\prime}\right]$. Let $h \in R$. Then $[h] \subseteq[R]$. We argue that $[h] \subseteq\left[R^{\prime}\right]$. Assume the opposite. Then, there is a play $p \in P$ such that $h \prec p$ and $p \notin\left[R^{\prime}\right]$. Since by Lemma 5.6.2, $\left[R^{\prime}\right]$ is clopen and so $P \backslash\left[R^{\prime}\right]$ is open, there exists a history $h^{\prime}$ with $h \preceq h^{\prime} \prec p$ and $\left[h^{\prime}\right] \cap\left[R^{\prime}\right]=\emptyset$. Thus, the set $\left[h^{\prime}\right]$ has the following properties: $(1)\left[h^{\prime}\right]$ is infinite because of our assumption that $|A| \geqslant 2,(2)\left[h^{\prime}\right] \subseteq[h] \subseteq[R]$ and $(3)\left[h^{\prime}\right] \cap\left[R^{\prime}\right]=\emptyset$. Then by using that $\mathrm{Q}_{1}, \mathrm{Q}_{1}^{\prime}, \mathrm{Q}_{2}$ and $\mathrm{Q}_{2}^{\prime}$ are finite sets, $\left([\mathrm{R}] \cup \mathrm{Q}_{1}\right) \backslash \mathrm{Q}_{2}$ and $\left(\left[\mathrm{R}^{\prime}\right] \cup \mathrm{Q}_{1}^{\prime}\right) \backslash \mathrm{Q}_{2}^{\prime}$ cannot be equal, contradicting (21). Hence, $[h] \subseteq\left[R^{\prime}\right]$. Because this holds for all $h \in R$, we conclude that $[R] \subseteq\left[R^{\prime}\right]$. For a similar reason, $[R] \supseteq\left[R^{\prime}\right]$. So, $[R]=\left[R^{\prime}\right]$.

Based on $[R]=\left[R^{\prime}\right]$, it follows easily by finiteness of $Q_{1}, Q_{2}, Q_{1}^{\prime}$ and $Q_{2}^{\prime}$ that $\mathrm{Q}_{1}=\mathrm{Q}_{1}^{\prime}$ and $\mathrm{Q}_{2}=\mathrm{Q}_{2}^{\prime}$.

Proof of Lemma 5.6.6: Fix an arbitrary behavior strategy b. It follows from $\psi^{s}(\mathrm{~b})$ being a charge on $\mathcal{R}^{s}$, and $\emptyset, \mathrm{P} \in \mathcal{R}^{s}$ that $\psi^{\mathrm{s}+\mathrm{s}}(\mathrm{b})(\emptyset)=0$ and $\psi^{\mathrm{s}+\mathrm{s}}(\mathrm{b})(\mathrm{P})$ $=1$.

Now we will show that $\psi^{s+s}(\mathrm{~b})$ is finitely additive on the algebra $\mathcal{R}^{s+s}$. Take two sets $Z, Z^{\prime} \in \mathcal{R}^{s+s}$ such that $Z \cap Z^{\prime}=\emptyset$. Due to Lemma 5.6.5, these sets have the following unique representations:

$$
\mathrm{Z}=\left([\mathrm{R}] \cup \mathrm{Q}_{1}\right) \backslash \mathrm{Q}_{2}
$$

where $[R] \in \mathcal{R}^{s}$ and $Q_{1}$ and $Q_{2}$ are finite subsets of $P$. Additionally, $[R] \cap Q_{1}=$ $\emptyset, \mathrm{Q}_{1} \cap \mathrm{Q}_{2}=\emptyset$ and $[\mathrm{R}] \supseteq \mathrm{Q}_{2}$. Similarly, for set $\mathrm{Z}^{\prime}$,

$$
Z^{\prime}=\left(\left[R^{\prime}\right] \cup Q_{1}^{\prime}\right) \backslash Q_{2}^{\prime}
$$

where $\left[R^{\prime}\right] \in \mathcal{R}^{s}$ and $Q_{1}^{\prime}$ and $Q_{2}^{\prime}$ are finite subsets of $P$. Additionally, $\left[R^{\prime}\right] \cap$ $\mathrm{Q}_{1}^{\prime}=\emptyset, \mathrm{Q}_{1}^{\prime} \cap \mathrm{Q}_{2}^{\prime}=\emptyset$ and $\left[\mathrm{R}^{\prime}\right] \supseteq \mathrm{Q}_{2}^{\prime}$.

We will show that $[R] \cap\left[R^{\prime}\right]=\emptyset$. Assume by contradiction that $[R] \cap\left[R^{\prime}\right] \neq \emptyset$. As $[R] \cap\left[R^{\prime}\right]$ is open, there exists a history $h \in H$ such that $[h] \subseteq[R] \cap\left[R^{\prime}\right]$. 
Since we assumed that $Z \cap Z^{\prime}=\emptyset$, we have $[h] \subseteq Q_{2} \cup Q_{2}^{\prime}$. However, the cylinder set $[h]$ is infinite (by the assumption that $|A| \geqslant 2$ ), while $Q_{2}$ and $Q_{2}^{\prime}$ are finite sets. We have reached a contradiction, and thus we can conclude that $[R] \cap\left[R^{\prime}\right]=\emptyset$.

It follows from $[R] \cap\left[R^{\prime}\right]=\emptyset$ that $Q_{2} \cap Q_{2}^{\prime}=\emptyset$. We can deduce from $Z \cap Z^{\prime}=\emptyset$ that $\mathrm{Q}_{1} \cap \mathrm{Q}_{1}^{\prime}=\emptyset$ as well.

Let us denote by $V=\left[R^{\prime}\right] \cap Q_{1}$ and by $V^{\prime}=[R] \cap Q_{1}^{\prime}$. Note that $V \subseteq Q_{2}^{\prime}$ and $V^{\prime} \subseteq Q_{2}$. The representation of $Z \cup Z^{\prime}$ is

$$
\mathrm{Z} \cup \mathrm{Z}^{\prime}=\left(\left([R] \cup\left[\mathrm{R}^{\prime}\right]\right) \bigcup\left(\left(\mathrm{Q}_{1} \backslash \mathrm{V}\right) \cup\left(\mathrm{Q}_{1}^{\prime} \backslash \mathrm{V}^{\prime}\right)\right)\right) \backslash\left(\left(\mathrm{Q}_{2} \backslash \mathrm{V}^{\prime}\right) \cup\left(\mathrm{Q}_{2}^{\prime} \backslash \mathrm{V}\right)\right) .
$$

Since $\mathcal{R}^{s}$ is an algebra, the set $[R] \cup\left[R^{\prime}\right] \in \mathcal{R}^{s}$. Moreover, $\left(\mathrm{Q}_{1} \backslash \mathrm{V}\right) \cup\left(\mathrm{Q}_{1}^{\prime} \backslash \mathrm{V}^{\prime}\right)$ and $\left(Q_{2} \backslash V^{\prime}\right) \cup\left(Q_{2}^{\prime} \backslash V\right)$ are finite subsets of $P$. Additionally, $\left([R] \cup\left[R^{\prime}\right]\right) \cap\left(\left(Q_{1} \backslash\right.\right.$ $\left.\mathrm{V}) \cup\left(\mathrm{Q}_{1}^{\prime} \backslash \mathrm{V}^{\prime}\right)\right)=\emptyset$, $\left(\left(\mathrm{Q}_{1} \backslash \mathrm{V}\right) \cup\left(\mathrm{Q}_{1}^{\prime} \backslash \mathrm{V}^{\prime}\right)\right) \cap\left(\left(\mathrm{Q}_{2} \backslash \mathrm{V}^{\prime}\right) \cup\left(\mathrm{Q}_{2}^{\prime} \backslash \mathrm{V}\right)\right)=\emptyset$ and $[R] \cup\left[R^{\prime}\right] \supseteq\left(Q_{2} \backslash V^{\prime}\right) \cup\left(Q_{2}^{\prime} \backslash V\right)$.

$$
\begin{aligned}
\psi^{s+s}(b) & \left(Z \cup Z^{\prime}\right)=\psi^{s}(b)\left([R] \cup\left[R^{\prime}\right]\right) \\
& +\sum_{p \in\left(Q_{1} \backslash V\right) \cup\left(Q_{1}^{\prime} \backslash V^{\prime}\right)} l^{s+s}(b, p)-\sum_{p \in\left(Q_{2} \backslash V^{\prime}\right) \cup\left(Q_{2}^{\prime} \backslash V\right)} l^{s+s}(b, p) \\
= & \psi^{s}(b)([R])+\psi^{s}(b)\left(\left[R^{\prime}\right]\right)+\sum_{p \in Q_{1}} l^{s+s}(b, p)-\sum_{p \in V} l^{s+s}(b, p) \\
& +\sum_{p \in Q_{1}^{\prime}} l^{s+s}(b, p)-\sum_{p \in V^{\prime}} l^{s+s}(b, p)-\sum_{p \in Q_{2}} l^{s+s}(b, p) \\
& +\sum_{p \in V^{\prime}} l^{s+s}(b, p)-\sum_{p \in Q_{2}^{\prime}} l^{s+s}(b, p)+\sum_{p \in V} l^{s+s}(b, p) \\
= & \psi^{s}(b)([R])+\psi^{s}(b)\left(\left[R^{\prime}\right]\right)+\sum_{p \in Q_{1}} l^{s+s}(b, p) \\
& +\sum_{p \in Q_{1}^{\prime}} l^{s+s}(b, p)-\sum_{p \in Q_{2}} l^{s+s}(b, p)-\sum_{p \in Q_{2}^{\prime}} l^{s+s}(b, p) \\
= & \psi^{s+s}(b)(Z)+\psi^{s+s}(b)\left(Z^{\prime}\right) .
\end{aligned}
$$

The first and last equalities follow from the definition of $\psi^{s+s}$ in (15). The second equality follows from $\psi^{s}$ being a charge on $\mathcal{R}^{s}$ and $[R] \cap\left[R^{\prime}\right]=\emptyset$. We also make use of $\mathrm{V} \subseteq \mathrm{Q}_{1}, \mathrm{~V} \subseteq \mathrm{Q}_{2}^{\prime}$ and similarly, $\mathrm{V}^{\prime} \subseteq \mathrm{Q}_{1}^{\prime}$ and $\mathrm{V}^{\prime} \subseteq \mathrm{Q}_{2}$. So we found that

$$
\psi^{s+s}(b)\left(Z \cup Z^{\prime}\right)=\psi^{s+s}(b)(Z)+\psi^{s+s}(b)\left(Z^{\prime}\right) .
$$


This means that $\psi^{s+s}(b)$ is indeed finitely additive for any behavior strategy b. Hence $\psi^{s+s}(b)$ is a charge for any behavior strategy $b$.

Proof of Lemma 5.6.7:

Proof of part 1. Let $b$ be a behavior strategy and $Z \in \mathcal{R}^{s}$. Then by (15) with $[R]=Z$ and $Q_{1}=Q_{2}=\emptyset$, we have $\psi^{s+s}(b)(Z)=\psi^{s}(b)(Z)$. Hence, by definition, $\psi^{s+s}(b)(Z)=\psi^{s}(b)(Z)$.

Proof of part 2. The proof is making use of the idea of Example 5.3.3. Take an action $a \in A$, and let $p=(a, a, \ldots) \in P$.

Consider the behavior strategy $b$ where $b(h)(a)=1$ for every $h \in H$. Let $h_{t}$ denote the history at period $t$ in which only action $a$ is played. Then by (15)

$$
\psi^{s+s}(b)(\{p\})=l^{s+s}(b, p)=\prod_{\mathfrak{t}=1}^{\infty} b\left(h_{t}\right)(a)=1 .
$$

Now we prove that there is a charge $\mu$ on $\mathcal{R}^{s+s}$ such that for every $Z \in \mathcal{R}^{s}$

$$
\psi^{s}(b)(Z)=\mu(Z) \text { and } \psi^{s}(b)(\{p\}) \neq \mu(\{p\}) .
$$

Note that $\left\{Z \in \mathcal{R}^{s}: Z \subseteq\{p\}\right\}=\{\emptyset\}$. So the inner approximation of $\{p\}$ by sets in $\mathcal{R}^{s}$ yields

$$
\sup _{\mathrm{Z} \in \mathcal{R}^{s}, \mathrm{Z} \subseteq\{\mathrm{p}\}} \psi^{\mathrm{s}}(\mathrm{b})(\mathrm{Z})=\psi^{\mathrm{s}}(\mathrm{b})(\emptyset)=0 .
$$

Based on Theorem 3.C. 3 there exists an extension $\mu \in<\mathrm{b} \mid \mathcal{R}^{s}, \psi^{s}>$ such that $\mu(\{p\})=0$. Since $\psi^{s+s}(b)(\{p\})=1 \neq 0=\mu(\{p\})$, this is exactly what we were looking for.

\section{Proof of Lemma 5.6.8:}

Part A: We show that $\mathcal{R}^{o} \supsetneq \mathcal{R}^{s+s}$.

Since each set in $\mathcal{R}^{s}$ is clopen by part 3 of Lemma 5.6.2 and since each singleton $\{p\}$, where $p \in P$, is closed, the algebra $\mathcal{R}^{o}$ includes $\mathcal{R}^{s}$ and each singleton $\{\mathrm{p}\}$. Hence, $\mathcal{R}^{\mathrm{o}} \supseteq \mathcal{R}^{\mathrm{s}+\mathrm{s}}$.

Now we prove that $\mathcal{R}^{\mathrm{o}} \neq \mathcal{R}^{s+s}$. We construct a set $Z \subset \mathrm{P}$ such that $Z \in \mathcal{R}^{\mathrm{o}}$ but $Z \notin \mathcal{R}^{s+s}$. The idea of the proof is related to Example 5.3.4.

Take two distinct actions $a$ and $a^{\prime}-$ recall that we assumed $|A| \geqslant 2$. For every $n \in \mathbb{N}$, let $p_{n}$ denote the play where action $a$ is played at the first $n$ periods, and action $a^{\prime}$ is played at all periods after that. Let $Z=\left\{p_{n}: n \in \mathbb{N}\right\}$. 
First we argue that $Z \notin \mathcal{R}^{s+s}$. Assume by way of contradiction that $Z \in \mathcal{R}^{s+s}$. Then by part 3 of Lemma 5.6.5, there are $[R] \in \mathcal{R}^{s}$ and finite subsets $Q_{1}$ and $\mathrm{Q}_{2}$ of $\mathrm{P}$ such that

$$
Z=\left([R] \cup Q_{1}\right) \backslash Q_{2},
$$

and $[R] \cap \mathrm{Q}_{1}=\emptyset, \mathrm{Q}_{1} \cap \mathrm{Q}_{2}=\emptyset$ and $[\mathrm{R}] \supseteq \mathrm{Q}_{2}$. Therefore,

$$
[R]=\left(Z \backslash Q_{1}\right) \cup Q_{2} .
$$

Since $Z$ is countably infinite and both $Q_{1}$ and $Q_{2}$ are finite, $[R]$ must be countably infinite too. This is however in contradiction with the fact that $[R]$ is open. Thus, $\mathbf{Z} \notin \mathcal{R}^{s+s}$ as claimed.

Now we argue that $Z \in \mathcal{R}^{o}$. Let $p_{\infty}=(a, a, \ldots)$. Note that the closure of $Z$ is $Z \cup\left\{p_{\infty}\right\}$. Since $Z \cup\left\{p_{\infty}\right\}$ and $\left\{p_{\infty}\right\}$ are closed, they are elements of the algebra $\mathcal{R}^{\circ}$. Thus $Z \in \mathcal{R}^{\circ}$ as well.

Part B: We show that $\mathcal{R}^{\circ}$ is always strictly smaller than the Borel sigmaalgebra of $\mathrm{P}$. We do it through constructing a set $\mathrm{Q}$ such that $\mathrm{Q}$ belongs to the Borel sigma-algebra of $\mathrm{P}$, but $\mathrm{Q} \notin \mathcal{R}^{\mathrm{o}}$. The proof is making use of the idea of Example 5.6.11.

We suppose for simplicity that there are only two actions: $A=\left\{a, a^{\prime}\right\}$ - the same set $\mathrm{Q}$ can be taken even if $A$ contains more actions. Consider the set $\mathrm{Q}$ of plays $p$ such that action $a^{\prime}$ only appears finitely many times in $p$. As $Q$ is countable, $\mathrm{Q}$ belongs to the Borel sigma-algebra of $\mathrm{P}$. However, we show below that $\mathrm{Q} \notin \mathcal{R}^{\mathrm{o}}$. In fact, we prove a stronger statement: there is a collection $y$ of subsets of $P$ such that $Q \in y$ and $y \cap \mathcal{R}^{o}=\emptyset$.

Let $y$ denote all subsets $Z$ of $P$ for which there is a non-empty open subset $\mathrm{O}$ of $\mathrm{P}$ with the following property: $\mathrm{Z}$ is dense in $\mathrm{O}$ and its complement $\mathrm{P} \backslash \mathrm{Z}$ is also dense in $O$, i.e., $Z \cap O^{\prime} \neq \emptyset$ and $(P \backslash Z) \cap O^{\prime} \neq \emptyset$ for every non-empty open set $\mathrm{O}^{\prime} \subseteq \mathrm{O}$. Note that $\mathrm{Q} \in \mathrm{y}-$ in fact, $\mathrm{Q}$ and $\mathrm{P} \backslash \mathrm{Q}$ are dense in all non-empty open subsets of $P$.

The collection $y$ has the following properties. (I) If $Z \in Y$, then by definition, $(P \backslash Z) \in Y$. (2) If $\left(Z_{1} \cup Z_{2}\right) \in y$, then $Z_{1} \in y$ or $Z_{2} \in y$. Indeed, suppose that $\left(Z_{1} \cup Z_{2}\right) \in Y$. Then there is a non-empty open subset $O$ of $P$ such that $Z_{1} \cup Z_{2}$ and $P \backslash\left(Z_{1} \cup Z_{2}\right)$ are both dense in $O$. 
Suppose first that $Z_{1}$ is dense in $O$. Then as $P \backslash Z_{1} \supseteq P \backslash\left(Z_{1} \cup Z_{2}\right)$, the set $P \backslash Z_{1}$ is also dense in $O$. Thus, $Z_{1} \in Y$ as desired.

Now suppose that $Z_{1}$ is not dense in $O$. Then there is a non-empty open set $\mathrm{O}^{\prime} \subseteq \mathrm{O}$ such that $\mathrm{Z}_{1} \cap \mathrm{O}^{\prime}=\emptyset$. Because $Z_{1} \cup Z_{2}$ is dense in $\mathrm{O}$, it also holds that $Z_{1} \cup Z_{2}$ is dense in $O^{\prime}$. As $Z_{1} \cap O^{\prime}=\emptyset$, we can conclude that $Z_{2}$ is dense in $\mathrm{O}^{\prime}$. In addition, because $P \backslash Z_{2} \supseteq P \backslash\left(Z_{1} \cup Z_{2}\right)$ and $P \backslash\left(Z_{1} \cup Z_{2}\right)$ is dense in $O$, the set $P \backslash Z_{2}$ is dense in $O$, and therefore $P \backslash Z_{2}$ is dense in $O^{\prime}$. In conclusion, $Z_{2} \in y$ as desired. Hence, we have shown that $y$ has properties (1) and (2).

All sets of the algebra $\mathcal{R}^{o}$ can be constructed from open sets by finitely many applications of the operations of taking unions of two sets and taking complements. More precisely, each set in $\mathcal{R}^{\mathrm{o}}$ can be written as a finite formula with only non-empty open sets and the operations union and complement. Now we prove that $y \cap \mathcal{R}^{\mathrm{o}}=\emptyset$. Suppose by contradiction that there is a set $Q^{\prime} \in y \cap \mathcal{R}^{o}$. Due to the above-mentioned finite formula for $Q^{\prime}$ and properties (1) and (2) for the set $y$, we can conclude that there is a non-empty open set that belongs to $y$. This is a contradiction. Hence, $y \cap \mathcal{R}^{o}=\emptyset$ indeed. In particular, $\mathrm{Q} \notin \mathcal{R}^{\mathrm{o}}$.

\section{Proof of Lemma 5.6.10:}

Proof of part 1. Take a behavior strategy $b$. We prove that $\psi^{\mathrm{o}}(\mathrm{b})$ agrees with $\psi^{s+s}(b)$ on all sets in $\mathcal{R}^{s}$ and all singletons $\{p\}$, where $p \in$ P. Because the algebra $\mathcal{R}^{s+s}$ is generated by these sets, we will then have the desired $\psi^{\mathrm{o}}(Z)=\psi^{s+s}(\mathrm{~b})(Z)$ for every $Z \in \mathcal{R}^{s+s}$.

First, let $Z \in \mathcal{R}^{\text {s }}$. By part 3 of Lemma 5.6.2, the set $Z$ is clopen in P. By the definition of $\psi^{\circ}(b)(Z)$, by (17) and by part 1 of Lemma 5.6.7

$$
\psi^{o}(b)(Z)=c^{o}(b)(Z)=\psi^{s}(b)(Z)=\psi^{s+s}(b)(Z) .
$$

Now let $p=\left(a_{1}, a_{2}, \ldots\right) \in P$, and consider the singleton $\{p\}$. Let $V=P \backslash\{p\}$.

Take $n \in \mathbb{N}$. Since $V$ is an open set and

$$
V \supset\left[A^{n} \backslash\left\{\left(a_{1}, \ldots, a_{n}\right)\right\}\right],
$$


we have

$$
\psi^{o}(b)(V) \geqslant \psi^{o}(b)\left(\left[A^{n} \backslash\left\{\left(a_{1}, \ldots, a_{n}\right)\right\}\right]\right)=1-\psi^{o}(b)\left(\left[\left(a_{1}, \ldots, a_{n}\right)\right]\right) .
$$

Hence, by letting $n$ converge to infinity, $\psi^{o}(b)(V) \geqslant 1-l^{s+s}(b, p)$.

Now we will show that the opposite inequality is also true: $\psi^{\mathrm{o}}(\mathrm{b})(\mathrm{V}) \leqslant$ $1-l^{s+s}(b, p)$. Take a $Z \subseteq V$ with $Z \in \mathcal{R}^{s}$. It follows from $\{p\}=P \backslash V$ that $p \in P \backslash Z$. Then $l^{s+s}(b, p) \leqslant \psi^{s+s}(b)(P \backslash Z)=\psi^{s}(b)(P \backslash Z)$. This is equivalent to $l^{s+s}(b, p) \leqslant 1-\psi^{s}(b)(Z)$, so $1-l^{s+s}(b, p) \geqslant \psi^{s}(b)(Z)$ for any $Z \subseteq V$ with $Z \in \mathcal{R}^{s}$. By (17) we have $1-l^{s+s}(b, p) \geqslant \psi^{o}(b)(V)$, as desired.

Based on the previous arguments, we have $\psi^{o}(b)(V)=1-l^{s+s}(b, p)$. Therefore,

$$
\psi^{o}(b)(\{p\})=l^{s+s}(b, p)=\psi^{s+s}(\{p\}) .
$$

Proof of part 2. Since $A$ is infinite, by renaming the actions we can assume that $\mathbb{N} \subseteq A$. The proof is making use of the idea of Example 5.3.5.

Consider the following behavior strategy $b$. The charge $b(\varnothing)$ is such that $\mathrm{b}(\varnothing)(\{n\})=0$ for every $\mathrm{n} \in \mathbb{N}$ and $\mathrm{b}(\varnothing)(\mathbb{N})=1$ (essentially, $\mathrm{b}(\varnothing)$ is a pure charge on $\mathbb{N}$ ), and for any other $h \in H$ the charge $b(h)$ is simply the Dirac measure on action 1 . For every $n \in \mathbb{N}$, let $p_{n}$ be a play which starts with $n$ at period 1 , and at all other periods is 1 . Let $V=\left\{p_{n}: n \in \mathbb{N}\right\}$.

Now we prove that there is a charge $\mu$ on $\mathcal{R}^{o}$ such that for every $Z \in \mathcal{R}^{s+s}$

$$
\psi^{\mathrm{o}}(\mathrm{b})(\mathrm{Z})=\mu(\mathrm{Z}) \text { and } \quad \psi^{\mathrm{o}}(\mathrm{V}) \neq \mu(\mathrm{V})
$$

When we revisited Example 5.3.5 under Approach IV in Section 5.7 we showed that $\psi^{\circ}(V)=1$. Let $V=\left\{Z \in \mathcal{R}^{s+s}: Z \subseteq V\right\}$. Since $V$ is countable and by (14), the elements of $\mathcal{V}$ are finite sets. Take a $Z \in \mathcal{V}$. After plugging in $l^{s+s}(b, p)=0$ for every play $p \in Z$ into (15) we arrive at $\psi^{s+s}(Z)=\sum_{p \in Z} l^{s+s}(b, p)=0$. So the inner approximation of $\mathrm{V}$ by sets in $\mathcal{R}^{s+s}$ yields

$$
\sup _{Z \in \mathcal{R}^{s+s}, Z \subseteq V} \psi^{s+s}(b)(Z)=0 .
$$

Based on Theorem 3.C. 3 there exists an extension $\mu \in<\mathrm{b} \mid \mathcal{R}^{s+s}, \psi^{s+s}>$ such that $\mu(V)=0$. Since $\psi^{o}(b)(V)=1 \neq 0=\mu(V)$, this is exactly what we were looking for. 
Proof of part 1 of Theorem 5.7.1: Let $\mathscr{P}$ be an algebra of $\mathrm{P}$, and $\psi^{\mathscr{P}}$ be a $\mathscr{P}-$ specification. Let a behavior strategy $b$ be given. We define a mixed strategy $\mathrm{m}$ as follows. Take an arbitrary charge $\mathrm{B} \in<\mathrm{b} \mid \mathscr{P}, \psi^{\mathscr{P}}>$. Let $\phi: \mathrm{P} \rightarrow \mathrm{F}$ be a function such that if $\phi(p)=f$ then $p_{f}=p$. This means that $\phi(p)$ is a pure strategy that induces the play $p$, for every $p \in P$. Note that $\phi$ is one-to-one, and $f \leftrightarrow p_{f}$ is a bijection between $\phi(P)$ and $P$.

For a set $\mathrm{G} \subseteq \mathrm{F}$, define

$$
m(G)=B\left(\left\{p_{f}: f \in G \cap \phi(P)\right\}\right) .
$$

Intuitively, $m(G)$ is the probability under B of the set of plays that are induced by a pure strategy in $G \cap \phi(P)$. We show in two steps that $m$ is a mixed strategy, i.e., that $m$ is a charge on $F$.

(1) Clearly, $m(\emptyset)=B(\emptyset)=0$ and $m(F)=B(P)=1$.

(2) Now we prove that $m$ is additive. Take two disjoint sets $G \subseteq F$ and $G^{\prime} \subseteq F$. Then, $\mathrm{G} \cap \phi(\mathrm{P})$ and $\mathrm{G}^{\prime} \cap \phi(\mathrm{P})$ are disjoint, and because $\phi$ is one-to-one, the sets $\left\{p_{f}: f \in G \cap \phi(P)\right\}$ and $\left\{p_{f}: f \in G^{\prime} \cap \phi(P)\right\}$ are also disjoint. Thus, by using the additivity of $B$, we obtain

$$
\begin{aligned}
m(G)+m\left(G^{\prime}\right) & =B\left(\left\{p_{f}: f \in G \cap \phi(P)\right\}\right)+B\left(\left\{p_{f}: f \in G^{\prime} \cap \phi(P)\right\}\right) \\
& =B\left(\left\{p_{f}: f \in\left(G \cup G^{\prime}\right) \cap \phi(P)\right\}\right) \\
& =m\left(G \cup G^{\prime}\right) .
\end{aligned}
$$

Thus, $m$ is a mixed strategy as claimed.

Finally, we argue that $\mathrm{m}$ and $\mathrm{b}$ are $\mathscr{P}$-equivalent. Take a set $\mathrm{Q} \in \mathscr{P}$. Let $\mathrm{G}=\left\{\mathrm{f} \in \mathrm{F}: \mathrm{p}_{\mathrm{f}} \in \mathrm{Q}\right\}$. Then $\left\{\mathrm{p}_{\mathrm{f}}: \mathrm{f} \in \mathrm{G} \cap \phi(\mathrm{P})\right\}=\mathrm{Q}$. In view of (8) we have

$$
\mathrm{m}^{*}(\mathrm{Q})=\mathrm{m}(\mathrm{G})=\mathrm{B}\left(\left\{\mathrm{p}_{\mathrm{f}}: \mathrm{f} \in \mathrm{G} \cap \phi(\mathrm{P})\right\}\right)=\mathrm{B}(\mathrm{Q})
$$

Thus, $\mathrm{m}$ and $\mathrm{b}$ are $\mathscr{P}$-equivalent as claimed.

Proof of part 2 of Theorem 5.7.1: Example 5.7.5 is the proof.

Proof of part 1 of Theorem 5.7.2: We prove that (1) implies (2) and that (2) implies (3). It follows from Lemma 5.5.5 that (3) implies (1) as well.

(2) implies (3): Assume that $u$ is uniformly continuous. Let $\varepsilon>0$. As we assume that $u$ is bounded, there is a finite set $K \subset \mathbb{R}$ such that for every 
play $p \in P$ there exists $k \in K$ with $|u(p)-k| \leqslant \varepsilon / 2$. Because $u$ is uniformly continuous, by Lemma 5.4.1, there is a $T \in \mathbb{N}$ such that if for two plays $p$ and $q$ it holds that $p(t)=q(t)$ for all $t \leqslant T$, then $|u(p)-u(q)| \leqslant \varepsilon / 2$. This means that for every history $h \in A^{\top}$ there is a number $z_{h} \in K$ such that for every $p \succ h$ we have $\left|u(p)-z_{h}\right| \leqslant \varepsilon$. Now we define a payoff function $\bar{u}$ by setting $\bar{u}(p)=z_{h}$ if $p \succ h$ for $h \in A^{\top}$. Thus $|\bar{u}(p)-u(p)| \leqslant \varepsilon$ for every $p \in P$. By construction, $\bar{u}$ is an $\mathcal{R}^{\text {hs }}$-measurable simple-function. Indeed,

$$
\overline{\mathrm{u}}(\mathrm{p})=\sum_{k \in K} k \cdot \mathbb{I}_{\left[\mathrm{Q}_{k}\right]}(p),
$$

where $Q_{k}=\left\{h \in A^{\top}: z_{h}=k\right\}$ for every $k \in K$.

(1) implies (2): We prove the contraposition of this implication. So assume that $u$ is not uniformly continuous. Then, by Lemma 5.4.1, there exists $\varepsilon>0$ for which for every $k \in \mathbb{N}$, there exists a history $h_{k} \in A^{k}$ and plays $p_{k} \succ h_{k}$ and $q_{k} \succ h_{k}$ such that $u\left(p_{k}\right)+\varepsilon<u\left(q_{k}\right)$. Consider the sequences of plays $\left(p_{k}\right)_{k \in \mathbb{N}}$ and $\left(q_{k}\right)_{k \in \mathbb{N}}$. In view of Lemma 5.B.1, by taking subsequences if necessary, we can assume that at least one of the following holds:

1. The sequences $\left(p_{k}\right)_{k \in \mathbb{N}}$ and $\left(q_{k}\right)_{k \in \mathbb{N}}$ converge to the same play as $k \rightarrow$ $\infty$.

2. There exists a history $h$ and a sequence of actions $\left(a_{k}\right)_{k \in \mathbb{N}}$, with $a_{k} \neq$ $a_{k^{\prime}}$ for all $k, k^{\prime} \in \mathbb{N}$ with $k \neq k^{\prime}$, such that $p_{k} \succ\left(h, a_{k}\right)$ and $q_{k} \succ\left(h, a_{k}\right)$ for every $k \in \mathbb{N}$.

Assume first that property 1 holds. (This case is illustrated in Example 5.3.3, with $p_{k}$ being a play starting with action $c$ up to period $k$ and action $s$ at period $k+1$, and each $q_{k}$ being the play $(c, c, \ldots)$.) Then $u\left(p_{k}\right)+\varepsilon<u\left(q_{k}\right)$ for every $k \in \mathbb{N}$ and both sequences converge to the same play, say to $\left(a_{1}, a_{2}, \ldots\right)$. Consider a behavior strategy $b$ such that $b\left(a_{1}, \ldots, a_{n-1}\right)$ is the Dirac measure on action $a_{n}$, for every $n \in \mathbb{N}$. We show that $U^{\text {hs }}(b)$ is not a singleton.

Let $\tau$ be a pure charge on $\mathbb{N}$. Define two charges $B_{p}, B_{q}$ on $P$ by for every $Z \subseteq P$ letting

$$
\begin{aligned}
& B_{p}(Z)=\int_{k \in \mathbb{N}} \delta_{p_{k}}(Z) \tau(d k), \\
& B_{q}(Z)=\int_{k \in \mathbb{N}} \delta_{q_{k}}(Z) \tau(d k) .
\end{aligned}
$$


It follows from Lemma 5.B.2 that $B_{p}, B_{q} \in<b \mid \mathcal{R}^{\text {hs }}, \psi^{\text {hs }}>$. Additionally, $u\left(B_{p}\right)+\varepsilon \leqslant u\left(B_{q}\right)$. Hence $u^{h s}(b)$ is not a singleton.

Now assume that property 2 holds. (This case is illustrated in Example 5.3.2, with $h=\varnothing$ and for every $k \geqslant 2: a_{k}=k, p_{k}=(k, k+1, k+1, \ldots)$ and $q_{k}=(k, k, \ldots)$.) Let $h_{k}$, for every $k \in \mathbb{N}$, be the longest history on which $p_{k}$ and $q_{k}$ coincide. Note that the length of $h_{k}$ converges to infinity.

Let $\tau$ be a pure charge on $\mathbb{N}$. Consider a behavior strategy $b$ that prescribes to play as follows: First, from the root, with Dirac measures follow $h$. Then at $h$ choose an action from $\left\{a_{1}, a_{2}, \ldots\right\}$ according to $\tau$. Finally, if action $a_{k}$ is chosen, then follow the play $p_{k}$ with Dirac measures on the corresponding actions.

Define two charges $\mathrm{B}_{\mathrm{p}}, \mathrm{B}_{\mathrm{q}}$ on $\mathrm{P}$ by for every $\mathrm{Z} \subseteq \mathrm{P}$ letting

$$
\begin{aligned}
& B_{p}(Z)=\int_{k \in \mathbb{N}} \delta_{p_{k}}(Z) \tau(d k), \\
& B_{q}(Z)=\int_{k \in \mathbb{N}} \delta_{q_{k}}(Z) \tau(d k) .
\end{aligned}
$$

It follows from Lemma 5.B.2 that $\mathrm{B}_{\mathrm{p}}, \mathrm{B}_{\mathrm{q}} \in<\mathrm{b} \mid \mathcal{R}^{\text {hs }}, \psi^{\text {hs }}>$. Additionally, $u\left(B_{p}\right)+\varepsilon \leqslant u\left(B_{q}\right)$. Hence $u^{\text {hs }}(b)$ is not a singleton.

Proof of part 2 of Theorem 5.7.2: We prove that (1) implies (2) and that (2) implies (3). It follows from Lemma 5.5.5 that (3) implies (1) as well.

(2) implies (3): Assume that $u$ is continuous. Let $\varepsilon>0$. Because $u$ is continuous, for every play $p \in P$, by part 1 of Lemma 5.4.I there is a prefix $h_{p} \in H$ such that in the subproblem at $h_{p}$ the payoff varies less than $\varepsilon$. Formally, for every two plays $q$ and $q^{\prime}$ that extend $h_{p}$ we have $\left|u(q)-u\left(q^{\prime}\right)\right|<\varepsilon / 2$. Let $h_{p}$ be the shortest such prefix of $p$. Note that for every play $q \succ h_{p}$ we have $h_{q}=h_{p}$. Let $S=\left\{h_{p}: p \in P\right\}$. Clearly, the set $S$ is a complete stop rule.

As we assume that $u$ is bounded, there is a finite set $K \subset \mathbb{R}$ such that for every play $p \in P$ there exists $k \in K$ with $|u(p)-k|<\varepsilon / 2$. By construction, for every history $h \in S$ there is a number $z_{h} \in K$ such that for every $p \succ h$ we have $\left|u(p)-z_{h}\right|<\varepsilon$. Now we define a payoff function $\bar{u}$ by setting $\bar{u}(p)=z_{h}$ if $p \succ h$ for $h \in S$. Thus $|\bar{u}(p)-u(p)|<\varepsilon$ for every $p \in P$. By construction, $\bar{u}$ is an $\mathcal{R}^{\mathrm{s}}$-measurable simple-function, as it has the form

$$
\bar{u}(p)=\sum_{k \in K} k \cdot \mathbb{I}_{\left[S_{k}\right]}(p),
$$


where $S_{k}=\left\{h \in S: z_{h}=k\right\}$ for every $k \in K$.

(1) implies (2): We prove the contraposition of this implication. So assume that $u$ is not continuous. Then $u$ is not continuous at some play $p=\left(a_{1}, a_{2} \ldots\right)$ $\in P$. This means that there are $\varepsilon>0$ and two sequences $\left(p_{k}\right)_{k \in \mathbb{N}}$ and $\left(q_{k}\right)_{k \in \mathbb{N}}$ of plays such that (1) both sequences $\left(p_{k}\right)_{k \in \mathbb{N}}$ and $\left(q_{k}\right)_{k \in \mathbb{N}}$ converge to $p$, (2) $u\left(p_{k}\right)_{k \in \mathbb{N}}$ converges to some $r_{p} \in \mathbb{R}$ and $u\left(q_{k}\right)_{k \in \mathbb{N}}$ converges to some $r_{q} \in \mathbb{R}$ such that $r_{p}+\varepsilon<r_{q}$.

Consider a behavior strategy $b$ such that $b\left(a_{1}, \ldots, a_{n-1}\right)$ is the Dirac measure on action $a_{n}$, for every $n \in \mathbb{N}$.

Let $\tau$ be a pure charge on $\mathbb{N}$. Define two charges $B_{p}, B_{q}$ on $P$ by for every $\mathrm{Z} \subseteq \mathrm{P}$ letting

$$
\begin{aligned}
& B_{p}(Z)=\int_{k \in \mathbb{N}} \delta_{p_{k}}(Z) \tau(d k), \\
& B_{q}(Z)=\int_{k \in \mathbb{N}} \delta_{q_{k}}(Z) \tau(d k) .
\end{aligned}
$$

It follows from Lemma 5.B.2 that $\mathrm{B}_{\mathrm{p}}, \mathrm{B}_{\mathrm{q}} \in<\mathrm{b}\left|\mathcal{R}^{s}, \psi^{s}\right\rangle$. Additionally, $\mathrm{u}\left(\mathrm{B}_{\mathrm{p}}\right)+$ $\varepsilon<u\left(B_{q}\right)$. Hence $U^{s}(b)$ is not a singleton.

Proof of part 3 of Theorem 5.7.2: Part 3-(a) follows from Lemma 5.5.5.

Now we prove part $3-(b)$. Assume that $u$ is strongly tame. We need to show that $u$ is uniformly $\mathcal{R}^{s+s}$-approachable. Let $\mathrm{D}_{\mathfrak{u}}$ denote the set of plays where $u$ is not continuous. Since $u$ is strongly tame, by part 1 of Lemma 5.4 .4 the function $L_{\mathfrak{u}}: P \rightarrow \mathbb{R}$ is continuous. Moreover, $L_{\mathfrak{u}}(p)=\mathfrak{u}(p)$ for all $p \in P \backslash D_{\mathfrak{u}}$. Note that $o_{\mathfrak{u}}(p)=\left|u(p)-L_{\mathfrak{u}}(p)\right|$ for each $p \in D_{\mathfrak{u}}$.

Let $\varepsilon>0$. Let $D_{\mathfrak{u}}^{\varepsilon}=\left\{p \in D_{\mathfrak{u}}: o_{\mathfrak{u}}(p)>\frac{\varepsilon}{2}\right\}$. Since $u$ is strongly tame, $D_{\mathfrak{u}}^{\varepsilon}$ is a finite set.

In view of part 2 of Theorem 5.7.2, applied to the continuous function $\mathrm{L}_{\mathrm{u}}$, there is an $\mathcal{R}^{s}$-measurable simple-function $\mathrm{g}: \mathrm{P} \rightarrow \mathbb{R}$ such that $\mid \mathrm{L}_{\mathfrak{u}}(\mathrm{p})-$ $g(p) \mid \leqslant \frac{\varepsilon}{2}$ for every $p \in P$. By $L_{\mathfrak{u}}(p)=u(p)$ for all $p \in P \backslash D_{\mathfrak{u}}$ and by $\mid L_{\mathfrak{u}}(p)-$ $\mathfrak{u}(p) \mid \leqslant \frac{\varepsilon}{2}$ for all $p \in D_{\mathfrak{u}} \backslash D_{\mathfrak{u}}^{\varepsilon}$, we obtain for all $P \backslash D_{\mathfrak{u}}^{\varepsilon}$ that $|u(p)-g(p)| \leqslant \varepsilon$. We define a function $\bar{u}: P \rightarrow \mathbb{R}$ by setting $\bar{u}(p)=g(p)$ for all $p \in D_{\mathfrak{u}} \backslash$ $D_{\mathfrak{u}}^{\varepsilon}$ and $\bar{u}(p)=\mathfrak{u}(p)$ for all $p \in D_{\mathfrak{u}}^{\varepsilon}$. Thus $|\bar{u}(p)-\mathfrak{u}(p)| \leqslant \varepsilon$ for every $p \in$ P. Since $g$ is an $\mathcal{R}^{s}$-measurable simple-function and $D_{\mathfrak{u}}^{\varepsilon}$ is a finite set, $\bar{u}$ is an $\mathcal{R}^{s+s}$-measurable simple-function. By construction and as mentioned 
earlier, $|u(p)-\bar{u}(p)| \leqslant \varepsilon$ for all $p \in P$. This proves that $u$ is uniformly $\mathcal{R}^{s+s_{-}}$ approachable.

\section{Proof of part 4 of Theorem 5.7.2:}

Part 4-(a): It follows from Lemma 5.5.5.

Part 4-(b)-i: Assume that $u$ is weakly tame. We prove that $u$ is uniformly $\mathcal{R}^{o}$ approachable. Let $\varepsilon>0$. Let $\mathrm{Q}$ denote the set of plays where the oscillation of $u$ is at most $\varepsilon / 2$, i.e., $Q=\left\{p \in P: o_{u}(p) \leqslant \varepsilon / 2\right\}$. Thus, $P \backslash Q$ is a finite set. By definition of $Q$, for every play $p \in Q$, there is a prefix $h_{p} \in H$ such that in the subproblem at $h_{p}$ the payoff varies at most $\varepsilon / 2$. Formally, for every two plays $\mathrm{q}, \mathrm{q}^{\prime} \in \mathrm{Q}$ that extend $\mathrm{h}_{\mathrm{p}}$ we have $\left|\mathrm{u}(\mathrm{q})-\mathrm{u}\left(\mathrm{q}^{\prime}\right)\right| \leqslant \varepsilon / 2$. Let $\mathrm{h}_{\mathrm{p}}$ be the shortest such prefix of $p$. Note that if a play $q \in Q$ extends $h_{p}$ then we have $h_{q}=h_{p}$. Let $S=\left\{h_{p}: p \in Q\right\}$.

As we assume that $u$ is bounded, there is a finite set $K \subset \mathbb{R}$ such that for every play $p \in P$ there exists $k \in K$ with $|u(p)-k|<\varepsilon / 2$. By construction, for every history $h \in S$ there is a number $z_{h} \in K$ such that for every $p \succ h$ we have $\left|u(p)-z_{h}\right|<\varepsilon$. Let $P_{k}=\cup_{h \in S, z_{h}=k}[h]$ for every $k \in K$. Note that $\left\{\mathrm{P}_{\mathrm{k}}: \mathrm{k} \in \mathrm{K}\right\}$ is a partition of $[\mathrm{S}]$. Now we define a payoff function $\bar{u}$ by setting $\bar{u}(p)=k$ if $p \in P_{k}$, and $\bar{u}(p)=u(p)$ if $p \in P \backslash Q$. Thus $|\bar{u}(p)-u(p)|<\varepsilon$ for every $p \in P$. Note that for all $k \in K$, the set $P_{k}$ is open and hence $P_{k} \in \mathcal{R}^{o}$. Moreover, since $\mathrm{P} \backslash \mathrm{Q}$ is finite, $\mathrm{P} \backslash \mathrm{Q} \in \mathcal{R}^{\mathrm{o}}$ as well. By construction, $\overline{\mathrm{u}}$ is an $\mathcal{R}^{o}$-measurable simple-function. Thus, $u$ is uniformly $\mathcal{R}^{o}$-approachable.

Parts 4-(b)-ii and 4-(b)-iii: Assume that $u$ is upper semi-continuous. We prove that $u$ is uniformly $\mathcal{R}^{\circ}$-approachable. Let $\varepsilon>0$. Choose $m \in \mathbb{N}$ such that $\frac{1}{m}<\varepsilon$. Since the payoff function $u$ is bounded, there exists $K \in \mathbb{N}$ such that $\mathrm{u}^{-1}([-\mathrm{Km}, \mathrm{Km}))=\mathrm{P}$.

Take an arbitrary $k \in\{-K,-K+1, \ldots, K-1, K\}$. Let $P_{k}=u^{-1}\left(\left[\frac{k}{m}, \frac{k+1}{m}\right)\right)$. For any $p, q \in P_{k}$, we have $|u(p)-u(q)|<\frac{1}{m}<\varepsilon$. Since $u$ is upper semicontinuous, the sets $u^{-1}\left(\left[\frac{\mathrm{k}}{\mathrm{m}}, \mathrm{Km}\right)\right)$ and $u^{-1}\left(\left[\frac{\mathrm{k}+1}{\mathrm{~m}}, \mathrm{Km}\right)\right)$ are closed, and hence $\mathrm{P}_{\mathrm{k}}$ is closed. Consequently, $\mathrm{P}_{\mathrm{k}} \in \mathcal{R}^{\mathrm{o}}$.

Now we define a payoff function $\bar{u}$ by setting $\bar{u}(p)=\frac{k}{m}$ if $p \in P_{k}$. Thus $|\bar{u}(p)-u(p)|<\varepsilon$ for every $p \in P$. By construction, $\bar{u}$ is an $\mathcal{R}^{o}$-measurable simple-function. Thus, $u$ is uniformly $\mathcal{R}^{\circ}$-approachable.

The proof of part $4-(b)$-iii is similar. 
Lemma 5.B.1. Consider two sequences of plays $\left(p_{k}\right)_{k \in \mathbb{N}}$ and $\left(q_{k}\right)_{k \in \mathbb{N}}$. For every $\mathrm{k} \in \mathbb{N}$, let $\mathrm{h}_{\mathrm{k}}$ denote the longest history on which $\mathrm{p}_{\mathrm{k}}$ and $\mathrm{q}_{\mathrm{k}}$ coincide. Assume that the length of $h_{k}$ converges to infinity as $k \rightarrow \infty$. Then, there exists an increasing sequence $\left(k_{\ell}\right)_{\ell \in \mathbb{N}}$ such that for the subsequences $\left(p_{k_{\ell}}\right)_{\ell \in \mathbb{N}}$ and $\left(q_{k_{\ell}}\right)_{\ell \in \mathbb{N}}$ at least one of the following holds:

1. The subsequences $\left(\mathrm{p}_{\mathrm{k}_{\ell}}\right)_{\ell \in \mathbb{N}}$ and $\left(\mathrm{q}_{\mathrm{k}_{\ell}}\right)_{\ell \in \mathbb{N}}$ converge to the same play as $\ell \rightarrow$ $\infty$.

2. There exists a history $\mathrm{h}$ and a sequence of actions $\left(a_{\ell}\right)_{\ell \in \mathbb{N}}$, with $\mathrm{a}_{\ell} \neq \mathrm{a}_{\ell^{\prime}}$ for all $\ell, \ell^{\prime} \in \mathbb{N}$ with $\ell \neq \ell^{\prime}$, such that $\mathrm{p}_{\ell} \succ\left(\mathrm{h}, \mathrm{a}_{\ell}\right)$ and $\mathrm{q}_{\ell} \succ\left(\mathrm{h}, \mathrm{a}_{\ell}\right)$ for every $\ell \in \mathbb{N}$.

Proof. For every $n \in \mathbb{N}$, let $\bar{A}_{n}$ denote the set of actions that is used at period $n$ by at least one of the plays in either one of the sequences $\left(p_{k}\right)_{k \in \mathbb{N}}$ and $\left(q_{k}\right)_{k \in \mathbb{N}}$. That is, if $p_{k n}$ and $q_{k n}$ denote the actions of $p_{k}$ and respectively $q_{k}$ at period $n$, then $\bar{A}_{n}=\cup_{k \in \mathbb{N}}\left\{p_{k n}, q_{k n}\right\}$. Thus, $p_{k}, q_{k} \in \times_{n=1}^{\infty} \bar{A}_{n}$ for every $k \in \mathbb{N}$. We distinguish two cases.

First assume that $\bar{A}_{n}$ is finite for every $n \in \mathbb{N}$. Then, the set $\times_{n=1}^{\infty} \bar{A}_{n}$ is a compact subset of $P$. This implies that there exists an increasing sequence $\left(k_{\ell}\right)_{\ell \in \mathbb{N}}$ in $\mathbb{N}$ such that the subsequence $\left(p_{k_{\ell}}\right)_{\ell \in \mathbb{N}}$ converges. Since the length of $h_{k_{\ell}}$ tends to infinity as $\ell \rightarrow \infty$, we conclude that both subsequences $\left(p_{k_{\ell}}\right)_{\ell \in \mathbb{N}}$ and $\left(q_{k_{\ell}}\right)_{\ell \in \mathbb{N}}$ converge to the same play. Thus, property 1 holds.

Now assume that, for some $n \in \mathbb{N}$, the sets $\bar{A}_{1}, \ldots, \bar{A}_{n-1}$ are finite, but $\bar{A}_{n}$ is infinite. Since by assumption the length of $h_{k}$ converges to infinity as $k \rightarrow \infty$, there exists $m \in \mathbb{N}$ such that the length of $h_{k}$ is at least $n+1$ for all $k \geqslant m$. In particular, $p_{k n}=q_{k n}$ for all $k \geqslant m$. Then, by finiteness of the set $\bar{A}_{1} \times \cdots \times \bar{A}_{n-1}$ and because $\bar{A}_{n}$ is infinite, there exists a history $h \in \bar{A}_{1} \times \cdots \times \bar{A}_{n-1}$ such that the set of actions

$$
\left\{p_{k n}: k \geqslant m \text { and } p_{k} \succ h\right\}=\left\{q_{k n}: k \geqslant m \text { and } q_{k} \succ h\right\}
$$

is infinite. It follows that there exists an increasing sequence $\left(k_{\ell}\right)_{\ell \in \mathbb{N}}$ such that $k_{1} \geqslant m, p_{k_{\ell}} \succ h$ and thus $q_{k_{\ell}} \succ h$ for all $\ell \in \mathbb{N}$, and moreover the action $p_{k_{\ell} n}=q_{k_{\ell} n}$, i.e. the common action in $p_{k_{\ell}}$ and $q_{k_{\ell}}$ at period $n$, is different for each value of $\ell$. Thus, property 2 is satisfied. 
Lemma 5.B.2. Take a sequence of plays $\left(p_{k}\right)_{k \in \mathbb{N}}$ which converges to the play $p=$ $\left(a_{1}, a_{2}, \ldots\right)$. Consider a behavior strategy $b$ such that the charge $b\left(a_{1}, \ldots, a_{n-1}\right)$ is a Dirac measure on action $\mathrm{a}_{\mathrm{n}}$, for every $\mathrm{n} \in \mathbb{N}$.

Let $\tau$ be a pure charge on $\mathbb{N}$. Define a charge $\mathrm{B}$ on $\mathrm{P}$ by for every $\mathrm{Z} \subseteq \mathrm{P}$ letting

$$
B(Z)=\int_{k \in \mathbb{N}} \delta_{p_{k}}(Z) \tau(d k)
$$

Then $\mathrm{B} \in<\mathrm{b} \mid \mathcal{R}^{\mathrm{s}}, \psi^{\mathrm{s}}>$, and hence $\mathrm{B} \in<\mathrm{b} \mid \mathcal{R}^{\text {hs }}, \psi^{\text {hs }}>$.

Proof. We need to show that B coincides with the charge $\psi^{s}(b)$ on algebra $\mathcal{R}^{s}$, i.e., $\mathrm{B}\left(\left.\mathrm{Q}\right|_{h}\right)=\psi^{s}\left(\left.\mathrm{~b}\right|_{h}\right)\left(\left.\mathrm{Q}\right|_{h}\right)$ for every $\mathrm{Q} \in \mathcal{R}^{s}$ and history $h$. Take $\mathrm{Q} \in \mathcal{R}^{s}$ and $h \in A^{n}$ where $n \in \mathbb{N}$, and let $h=\left(a_{1}, \ldots, a_{n}\right)$ be the prefix of $p$ up to period $n$. Since $\left(p_{k}\right)_{k \in \mathbb{N}}$ converges to the play $p$, there exists $N \in \mathbb{N}$ such that $h \prec p_{k}$ for any $k \geqslant N$. We distinguish two cases.

First, assume that $p \in Q \cap[h]$. Then $\psi^{s}\left(\left.b\right|_{h}\right)\left(\left.Q\right|_{h}\right)=1$. Moreover, $h_{n}=h$ and $[h] \subseteq \mathrm{Q}$. Thus, for all $k \geqslant N$, the play $\mathrm{p}_{\mathrm{k}} \in[\mathrm{h}] \subseteq \mathrm{Q}$. Hence, $\delta_{\mathrm{p}_{\mathrm{k}}}(\mathrm{Q} \cap[\mathrm{h}])=1$. Since $\tau$ is a pure charge on $\mathbb{N}$,

$$
\mathrm{B}(\mathrm{Q} \cap[\mathrm{h}])=\int_{k \in \mathbb{N}} \delta_{p_{k}}(\mathrm{Q} \cap[h]) \tau(\mathrm{dk})=\int_{k \geqslant N} \delta_{p_{k}}(\mathrm{Q} \cap[h]) \tau(\mathrm{dk})=1 .
$$

Now assume that $p \notin Q \cap[h]$. Then $\psi^{s}\left(\left.b\right|_{h}\right)\left(\left.Q\right|_{h}\right)=0$. Moreover, $\left[h_{n}\right] \cap(Q \cap$ $[h])=\emptyset$. Thus, for all $k \geqslant N$, the play $p_{k} \in\left[h_{n}\right]$ and $p_{k} \notin Q \cap[h]$. Hence, $\delta_{p_{k}}(Q \cap[h])=0$. Since $\tau$ is a pure charge on $\mathbb{N}$,

$$
B(Q \cap[h])=\int_{k \in \mathbb{N}} \delta_{p_{k}}(Q \cap[h]) \tau(d k)=\int_{k \geqslant N} \delta_{p_{k}}(Q \cap[h]) \tau(d k)=0 .
$$

So, $B(Q \cap[h])=\psi^{s}\left(\left.b\right|_{h}\right)\left(\left.Q\right|_{h}\right)$ in both cases. By this, and $Q$ and $h$ being arbitrarily chosen, we have $B \in<b \mid \mathcal{R}^{s}, \psi^{s}>$. It follows from Lemma 5.6.4 that $\mathrm{B} \in<\mathrm{b} \mid \mathcal{R}^{\text {hs }}, \psi^{\text {hs }}>$ as well. 



\section{CONCLUSIONS}

This thesis investigates how different models of probabilities have an effect on games. We consider finitely and countably additive probabilities, which can be defined on different (sigma-)algebras. We also investigate how different set theoretic axioms can have an effect on these models.

Across the different chapters we consider games where it makes a difference which model of probability we take. More specifically, Chapters 3 and 4 contain zero-sum games with infinite action spaces, and Chapter 5 contains a game with infinitely many time periods.

In Chapter 3 we focus on finitely additive strategies defined on the power set of the action space. We consider different approaches of defining the expected payoff for a given strategy profile. This gives rise to multiple types of guarantee levels that we relate to each other. Finally, for a countable action space, we also compare these guarantee levels to guarantee levels defined through countably additive strategies defined on the power set.

In Chapter 4 we shift our focus to countably additive strategies. Unlike in the previous chapter, we also consider games with uncountable action spaces and with countably additive strategies. In this case similar problems arise to the finitely additive case, the expected payoff for a strategy profile might not be uniquely defined. We investigate how the guarantee levels of a catch game depend on the cardinality of the action space, the sigma-algebra and on set theoretic axioms. Finally, we also relate these guarantee levels to the finitely additive ones. In Chapter 3 we only consider strategies that are defined on the power set, but in Chapter 4 we also consider strategies defined on different sigma-algebras. In Chapter 3 for finitely additive strategies defined on the power set it does not make a difference what the cardinality of the action space is, til it is infinite. However, in Chapter 4 for countably additive strategies the cardinality of the action space is also relevant. 
In Chapter 5 we consider a special type of game with one player, a decision problem. In the previous chapters for the different models of probability to make a difference, we need an infinite action space. However, in this chapter some of the approaches are different even if the action space is finite. The difference comes from having infinitely many time periods instead of only one, as in the previous chapters. In this case it is not trivial how to define the expected payoff for a finitely additive behavior strategy. 


\section{BIBLIOGRA PHY}

Abaffy, M, Brázdil, T, Řehák, V, Bošanský, B, Kučera, A and Krčál, J (2014) Solving adversarial patrolling games with bounded error: (extended abstract). In Proceedings of the 2014 international conference on Autonomous agents and multi-agent systems (AAMAS '14). International Foundation for Autonomous Agents and Multiagent Systems, Richland, SC, 1617-1618.

Aliprantis, D and Border, KC (2005) Infinite Dimensional Analysis. Springer, Berlin.

Aryal, G and Stauber, R (2014) A note on Kuhn's Theorem with ambiguity averse players. Economic Letters, vol 125, issue 1, 110-114.

Aumann, RJ (1974) Subjectivity and correlation in randomized strategies. Journal of Mathematical Economics, vol 1, no 1, 67-96.

Bingham, NH (2010) Finite additivity versus countable additivity. Electronic Journal for History of Probability and Statistics, vol 6, no 1.

Blackwell, D (1942) Idempotent Markoff Chains, Annals of Mathematics, Second Series, vol 43, no 3, 560-567.

Capraro, V and Scarsini, M (2013) Existence of equilibria in countable games: An algebraic approach. Games and Economic Behavior, 79, issue C, 163180 .

Crawford, VP and Iriberri, M (2007) Fatal Attraction: Salience, Naïveté, and Sophistication in Experimental "Hide-and-Seek" Games, American Economic Review, 97(5), 1731-1750.

Dubins, LE (1974) On Lebesgue-like extensions of finitely additive measures. The Annals of Probability, vol 2, no 3, 456-463.

Dubins, LE and Savage, LJ, edited and updated by Sudderth, WD and Gilat, D (2014) How to gamble if you must: inequalities for stochastic processes. Dover Publications, New York. 
Dunford, N and Schwartz, JT (1964) Linear Operators, Part I: General Theory. Interscience Publishers, New York.

de Finetti, B (1975) The theory of probability (2 volumes). J. Wiley and Sons, Chichester.

Flesch, J, Vermeulen, D, and Zseleva, A (2017) Zero-sum games with charges. Games and Economic Behavior, 102, 666-686.

Gale, D and Stewart, FM (1953) Infinite games with perfect information, Contributions to the theory of games, vol 2, Annals of Mathematics Studies, no 28, Princeton University Press, 245-266.

Gilboa, I, Postlewaite, A, Samuelson, L and Schmeidler, D (2014) Economic Models as Analogies. The Economic Journal, vol 124, no 2, 513-533.

Gilboa, I and Schmeidler, D (1989) Maxmin Expected Utility with NonUnique Prior. Journal of Mathematical Economics, Elsevier, vol 18, no 2, 141-153.

Harris, JH, Stinchcombe, MB, and Zame, WR (2005) Nearly compact and continuous normal form games: characterizations and equilibrium existence. Games and Economic Behavior, 50, 208-224.

Heath, D and Sudderth, W (1972) On a Theorem of de Finetti, oddsmaking and game theory. Annals of Mathematical Statistics, 43, 2072-2077.

Isaacs, R (1965) Differential Games. John Wiley and Sons, New York.

Jech, T (2006) Set Theory, The Third Millenium Edition, revised and expanded. Springer, New York.

Kechris, AS (1995) Classical Descriptive Set Theory, Springer-Verlag.

Kiekintveld, C, Jain, J, Tsai, J, Pita, J, Ordonez, F and Tambe, M (2009) Computing optimal randomized resource allocations for massive security games, Proceedings of the Eighth International Joint Conference on Autonomous Agents and Multi-Agent Systems (AAMAS), Budapest, Hungary, 689-696.

Kindler, J (1983) A general solution concept for two-person zero sum games. J. Optimization Theory and Applications 40, 105-119. 
Lehrer, E (2009) A new integral for capacities. Economic Theory 39, 157-176

Loś, J and Marczewski, E (1949) Extensions of measure. Fundamenta Mathematicae, issue $36,267-276$.

Maitra, A and Sudderth, W (1993) Finitely additive and measurable stochastic games. International Journal Game Theory 22, 201-223.

Maitra, A and Sudderth, W (1998) Finitely additive stochastic games with Borel measurable payoffs. International Journal Game Theory 27, 257-267.

Marinacci, M (1997) Finitely additive and epsilon Nash equilibria. International Journal of Game Theory, 26, issue 3, 315-333.

Martin, DA (1975) Borel Determinacy, Annals of Mathematics, Second Series, vol 102, no 2, 363-371.

Maschler, M, Solan, E and Zamir, S (2013) Game Theory. Cambridge University Press.

Muraviev, I, Riedel, F and Sass, L (2017) Kuhn's Theorem for extensive form Ellsberg games. Journal of Mathematical Economics, 68, 26-41.

Mycielski, J and Świerczkowski, S (1964) On the Lebesgue measurability and the axiom of determinateness. Fundamenta Mathematicae 54-1, 67-71.

Lévy, A and Solovay, RM (1967) Measurable cardinals and the continuum hypothesis. Israel Journal of Mathematics 5, 234-248.

Oxtoby, JC (1980) Measure and Category: A Survey of the Analogies between Topological and Measure Spaces, Second Edition. Springer-Verlag, New York.

Peters, $\mathrm{H}$ and Vermeulen, D (2012) WPO, COV and IIA bargaining solutions for non-convex bargaining problems. International Journal of Game Theory, 41, issue 4, p. 851-884.

Pivato, M (2014) Additive representation of separable preferences over infinite products. Theory and Decision, 77, issue 1, 31-83.

Purves, R and Sudderth, W (1976) Some finitely additive probability. Annals of Probability, 4, 259-276. 
Prikry, K and Sudderth, WD (2016) Measurability of the value of a parametrized game. International Journal of Game Theory, vol 45, 675-683.

Rao, KPSB and Rao, B (1983) Theory of charges: a study of finitely additive measures. Academic Press, New York.

Reny, PJ (1999) On the Existence of Pure and Mixed Strategy Nash Equilibria in Discontinuous Games. Econometrica, vol 67, no 5, 1029-1056.

Rubinstein, A, Tversky, A and Heller, D (1996) Naive Strategies in Competitive Games, in Understanding Strategic Interaction - Essays in Honor of Reinhard Selten, W Guth et al (editors), Springer-Verlag, 394-402.

Savage, LJ (1972) The foundations of statistics. Dover Publications, New York.

Schervish, MJ and Seidenfeld, T (1996) A fair minimax theorem for twoperson (zero-sum) games involving finitely additive strategies. In: Berry, D.A., Chaloner, KM, Geweke, JK (Eds.), Bayesian Analysis in Statistics and Econometrics. Wiley, New York, 557-568.

Schirokauer, O and Kadane, J B (2007) Uniform distributions on the natural numbers. Journal of Theoretical Probability, 20, issue 3, 429-441.

Schmeidler, D (1989) Subjective Probability and Expected Utility without Additivity. Econometrica, 57, 571-587.

Sierpiński, W (1922) Sur les fonctions d'ensemble additives et continues, Fund. Math. 3, 240-246.

Solovay, RM (1971) Real-valued measurable cardinals. Axiomatic set theory (Proc. Sympos. Pure Math., vol XIII, Part I, Univ. California, Los Angeles, Calif., 1967), 397-428. Amer. Math. Soc., Providence, R.I.

Sudderth, W (2016) Finitely additive dynamic programming. Mathematics of Operations Research, 41, 92-108.

Takahashi, M (1969) A generalization of Kuhn's theorem for an infinite game. J. Sci. Hiroshima Univ. Ser. A-I Math. 33, no 2, 237-242.

von Neumann, J (1928) Zur Theorie der Gesellschaftsspiele. Mathematische Annalen, 100, 295-320. 
Wald, A (1945) Generalization of a theorem by v. Neumann concerning zero sum two person games. Ann. of Math., 2, issue 46, 281-286.

Wald, A (1950) Statistical Decision Functions. Wiley, New York.

Yanovskaya, EB (1970) The solution of infinite zero-sum two-person games with finitely additive strategies. Theory of Probability and Its Applications, vol 15, no 1, 153-158.

Zame, WR (2007) Can intergenerational equity be operationalized?, Theoretical Economics 2, 187-202. 



\section{INDEX}

$2^{X}, 11$

$<\mu_{1}, \mu_{2}>, 13$

$\mathrm{B}_{\mathrm{m}, 49}$

$\mathrm{D}_{\mathrm{g}}, 89$

$\mathrm{G}\left(\mathrm{F}_{1}, \mathrm{~F}_{2}\right), 2 \mathrm{I}$

$\mathrm{H}, 8 \mathrm{O}$

$\mathrm{L}_{\mathrm{g}}(\mathrm{p}), 88$

$\mathrm{P}, 8 \mathrm{O}$

$\mathrm{Q}(\mathrm{I}, \mathrm{m}), 49$

$\mathrm{S} \mid \mathrm{a}, 116$

$\mathrm{U}\left(<\mu_{1}, \mu_{2}>\right), 19$

$\mathrm{U}(\mu), 13$

$\mathrm{U}\left(\mathrm{b} \mid \mathscr{P}, \psi^{\mathscr{P}}\right), 92$

$\mathrm{U}(\mathrm{f}), 90$

$\mathrm{U}(\mathrm{m}), 90$

$\mathrm{U}^{\mathrm{o}}\left(\mu_{1}, \mu_{2}\right), \mathrm{I}_{5}$

$\mathrm{u}^{\mathrm{p}}\left(\mu_{1}, \mu_{2}\right), 15$

$\mathrm{U}^{12}\left(\mu_{1}, \mu_{2}\right), 16$

$\mathrm{U}^{21}\left(\mu_{1}, \mu_{2}\right), 15$

$\mathrm{u}^{\mathrm{hs}}(\mathrm{b}), 94$

$\mathrm{u}^{\mathrm{o}}(\mathrm{b}), 99$

$\mathrm{u}^{\mathrm{s}+\mathrm{s}}(\mathrm{b}), 98$

$\mathrm{u}^{\mathrm{s}}(\mathrm{b}), 96$

$\mathrm{V}=\mathrm{L}, 74$

$\mathrm{Y}(\mathrm{D}, \mathrm{k}), 5 \mathrm{O}$

[h], 88

$\mathcal{D}\left(A_{i}\right), 20$

$\Delta(\mathrm{X}), 12$

$\Delta(X, \mathscr{P}), 5$
$\mathcal{F}(\mathrm{X}), 11$

$\mathbb{N}, 12$

$Q(X), 12$

$\mathcal{R}^{\circ}, 98$

$\mathcal{R}^{s}, 94$

$\mathcal{R}^{\text {hs }}, 91$

$\mathcal{R}^{s+s}, 96$

$\mathscr{P}$-equivalent strategies, 92

$\mathscr{P}$-measurable simple-function, 6

$\mathscr{P}$-specification, 92

$\delta_{x}, 6$

$\mathbb{I}_{\mathrm{B}}, 6$

$\mathrm{e}(\mathrm{X}), 12$

$\mathcal{C}(\mathrm{X}, \mathscr{P}), 5$

$\mathcal{F}(A), 49$

| A |, 47

$\psi\left(\left.b\right|_{h}\right)\left(\left.Q\right|_{h}\right), 95$

$\psi^{\text {hs }}, 93$

$\psi^{s+s}, 97$

$\psi^{s}, 95$

$\tau$-guarantee level, 19

$\tau$-optimal strategy, 24

$\tau$-value, 19

21-guarantee level, 20

$c^{\text {hs }}(b)([R]), 91$

$c^{\mathrm{o}}(\mathrm{b})(\mathrm{O}), 99$

$\mathrm{h} \prec p, 86$

$h(t), 86$

$l^{s+s}(b, p), 97$ 
$\mathrm{m}^{*}(\mathrm{Q}), 90$

$\mathrm{o}_{\mathrm{g}}(\mathrm{p}), 88$

$u(B), 92$

$v\left(F_{1}, F_{2}\right), 21$

$v^{\tau}, 19$

$v_{1}, 22$

$v_{1}^{21}, 20$

$v_{1}^{\mathrm{d}}, 21$

$v_{1}^{f}, 21$

$v_{1}^{\mathrm{o}}, 20$

$v_{1}^{\mathrm{p}}, 2 \mathrm{O}$

$v_{1}^{\tau}, 19$

$v_{1}^{12}, 20$

$v_{2}, 22$

$v_{2}^{21}, 20$

$v_{2}^{\mathrm{d}}, 21$

$v_{2}^{f}, 21$

$v_{2}^{\mathrm{o}}, 2 \mathrm{O}$

$v_{2}^{\mathrm{p}}, 20$

$v_{2}^{\tau_{2}}, 19$

$v_{2}^{12}, 20$

$w_{1}^{\mathrm{o}}, 5 \mathrm{O}$

$w_{1}^{\mathrm{p}}, 5 \mathrm{O}$

$w_{2}^{\mathrm{o}}, 5 \mathrm{O}$

$w_{2}^{\mathrm{p}}, 5 \mathrm{O}$

12-optimal strategy, 30

21-optimal strategy, 30

algebra, 5

Approach I, 93

Approach II, 94

Approach III, 96

Approach IV, 98

atomless, 48

Axiom of Choice, 72
Axiom of Determinacy, 72

behavior strategy, 81

binary games, 12

catch game, 48

charge, 5

classical guarantee level, 22

competent algebra, 92

complete stop rule, 87

constructable set, 74

continuous function, 86

Continuum Hypothesis, 73

convergence, 86

countably additive function, 47

countably additive probability

measure, 5

countably infinite zero-sum game,

22

cover, 48

cylinder set, 88

Dirac measure, 6

expected payoff, 13

expected payoff induced by a behavior strategy, 92

expected payoff induced by a mixed strategy, 90

extension, 13

extension function, 13

Fubini-equality, 16

Generalized Continuum

Hypothesis, 73

history, 8o 
horizontal stop rule, 87

integrable, 21

layer, 58

lower semi-continuous function, 88

Measurable Cardinals, 74

minimalistic, 59

mixed strategy, 81

non-trivial measure, 48

optimistic, 14

optimistic guarantee level, 20

optimistic optimal strategy, 24, 51

optimistic utility, 15

outer measure, 48

pessimistic, 15

pessimistic guarantee level, 20

pessimistic optimal, 51

pessimistic optimal strategy, 28

pessimistic utility, 15

play, 80

probability charge, 5 probability measure, 5

pure charge, 6

pure strategy, 81

rectangle, 13, 48

removable discontinuity, 89

sigma-algebra, 5

simple-function, 21

stop rule, 87

strategy profile, 13

strongly tame function, 89

subproblem, 94

uniformly $\mathscr{P}$-approachable function, 92

uniformly continuous function, 86 upper semi-continuous function, 88

weakly tame function, 88

winning set, 12

Zermelo Fraenkel axiom system, 72

zero-sum game, 12 

A noncooperative game is a model of strategic interaction between players. A player's payoff might depend on the decisions taken by other players. Decision theory can be viewed as a special case of game theory where there is only one player, called a decision maker. Game theory has many applications in economics, biology, computer science, logic, political science and psychology. ZERO-SUM GAMES In zero-sum games there are two players. Given any pair of strategies of the players, the payoffs of the two players add up to zero. Hence, it is sufficient to consider the payoff of player 1 and also interpret it as the payment of player 2. These types of games are also called strictly competitive or conflict games.

The value is the central solution concept of zero-sum games. If a zero-sum game has a value, then this is the highest amount that player 1 can guarantee to receive, irrespective of what the other player plays. Similarly, the value is the lowest amount that player 2 can guarantee to pay, irrespective of what player 1 plays.

The theory of zero-sum games starts with von Neumann (1928), who showed that zero-sum games with finite action spaces admit a value. Games with infinite action spaces are much more complex, and Wald (1945) demonstrated that such games do not always have a value. In Wald's game there are two players who can each say any natural number. Player 1 wins, if he says a higher number or the same as player 2, otherwise he loses.

pRobabilities When a player makes a choice, he bases his decision on probabilities. In game theory the usual approach is to define mixed strategies as countably additive probabilities on the actions. A notable, but less frequent alternative is to define mixed strategies as finitely additive probabilities, socalled charges. Since finite additivity is a weaker requirement than countable additivity, the latter approach allows for a richer class of mixed strategies. Charges have regularly been argued for from a conceptual point of view, but they also provide technical advantages. 
THESIS This thesis investigates how different models of probabilities have an effect on games. More specifically, Chapters 3 and 4 contain zero-sum games with infinite action spaces, and Chapter 5 contains a game with infinitely many time periods. In Chapter 3 we consider any game with a bounded payoff function, and mainly focus on finitely additive strategies. In Chapter 4 we consider only a certain type of zero-sum games, and focus on countably additive strategies.

In Chapter 3 we consider two-player zero-sum games with infinite action spaces and bounded payoff functions. The players' strategies are finitely additive probability measures, called charges. Since a strategy profile does not always induce a unique expected payoff, we distinguish two extreme attitudes of players. A player is viewed as pessimistic if he always evaluates the range of possible expected payoffs by the worst one, and a player is viewed as optimistic if he always evaluates it by the best one. This approach results in a definition of a pessimistic and an optimistic guarantee level for each player. We provide an extensive analysis of the relation between these guarantee levels, and connect them to the guarantee levels defined through countably additive strategies, and to other known techniques to define expected payoffs, based on computation of double integrals. In addition, we also examine existence of optimal strategies with respect to these guarantee levels.

Chapter 3 is centered around the problem that a finitely additive strategy profile does not always induce a unique expected payoff. One might reach the conclusion that finitely additive strategies are problematic, and so their use should be avoided. In Chapter 3 we only compute the guarantee levels through countably additive strategies when the action space is countable. When a game has action spaces with larger cardinalities, countably additive strategies can cause similar problems to finitely additive strategies. This is investigated in Chapter 4.

In Chapter 4 we examine the guarantee levels defined through countably additive strategies of the players in a type of zero-sum games, catch games. We show how these levels depend on the sigma-algebras that are being employed on the player's action spaces. We further argue that guarantee levels may therefore also depend on set theoretic considerations. Additionally, we calculate the guarantee levels for finitely additive strategies. The solutions of 
catch games essentially differ among these setups. We find optimal strategies for almost all cases.

In Chapters 3 and 4 we consider two-player games, however Chapter 5 includes a game with one player, so a decision theoretic model. Chapters 3 and 4 consider games where players move once and simultaneously. In Chapter 5 the decision maker is taking decisions over infinitely many time periods.

In Chapter 5 we consider the following class of decision problems. A decision maker chooses an action $a_{t}$ from a given action space $A$ at every period $t=1,2, \ldots$, and receives a payoff that is a function of the resulting sequence $\left(a_{1}, a_{2}, \ldots\right)$. A mixed strategy of the decision maker is a finitely additive probability measure on the space of pure strategies. A behavior strategy of the decision maker is a mapping that assigns to every history a finitely additive probability measure on the action space. In this setup, we address several questions, both from a conceptual and from a technical point of view. For a behavior strategy, it is generally not clear which finitely additive measure such a strategy induces on the set of plays, i.e., on the set of all infinite sequences of elements of $A$. Consequently, it is not clear which mixed strategies are equivalent to the given behavior strategy, and therefore what the expected payoff should be. We present and compare various approaches to this problem. Moreover, we investigate the equivalence between behavior and mixed strategies. 



\section{VALORISATION}

In general, the Valorization addendum of a PhD thesis seems to be a controversial topic. On one hand, it is valuable to make research more accessible to lay people, both through open access publications and through making research output more understandable. On the other hand, the tendency to marginalize fundamental research is distressing. If the purpose of science is to accumulate knowledge, then fundamental research should also be considered useful. Looking for direct applications is complicated, since the fruit of this type of research can come after a considerable amount of time and moreover, indirectly. This is why there are projects that seemed useless at some point, but are currently considered indispensable. Even at the onset of a research project, one might not know what will be the end result, for example, some famous discoveries were made accidentally.

Specifically, this Valorization addendum creates an opportunity to think about why I spent years of my life being a $\mathrm{PhD}$ candidate, and why tax payers supported me in this project. Thinking about usefulness can raise a lot of philosophical questions, for example whether humanity is useful on its own. Assuming that we should keep up the human race, what is useful for it is still an intriguing question which would lead us far away. I will build on a more pragmatic basis.

I start by assuming that education is useful, as it is usually subsidized by the public sector in most European countries. Since I taught a number of courses, I contributed to others' education. Moreover, a $\mathrm{PhD}$ is a part of my own personal education. As a $\mathrm{PhD}$ candidate, one does not only learn a lot about their own subfield, but through colleagues one can get familiarized with other fields as well.

This thesis is part of fundamental research, so direct applications are meant for researchers. The focus of this thesis is on mixed strategies. Mixed strategies are part of both decision theory and game theory, which means that we assume that decision makers or players can take probabilistic decisions. The 
results of this thesis are relevant for models where the time horizon or the action space is infinite.

When you do not know clearly when a game ends, then it can be modeled with an infinite time horizon. For example, discounted models in macroeconomics or bargaining theory can have infinite time horizons. There are numerous models in economics with infinitely many actions. In basic models of industrial organization, firms are assumed to choose from an interval of prices. Similarly, bids and valuations in auction theory are often assumed to be infinite. In spatial economics, for example, in Hotelling's model firms can choose from an infinite set of locations. Consider a stretch of beach with sun bathers and two ice cream vendors. Since the vendors serve the same ice cream at the same price, consumers simply go to the closest vendor. The prediction of this model is that both ice cream vendors will locate in the middle of the stretch of beach, minimizing their product differentiation. Hotelling's model has also been used to analyse many other situations, including radio station programs and political candidates' platforms.

One of the lessons of this thesis is to be careful when modeling games of these types, because they can be very sensitive even to seemingly innocuous changes.

I have argued so far that the results of this thesis can be interesting to researchers in (applied) game theory. As I argued in Chapter 1, game theory has numerous applications to other fields, including economics. Time is well spent on improving economic models, since economists are often part of decision processes that have a vast impact on society. The most famous examples are Nobel-prize winners. For example, Jean Tirole advises the French government, and Alvin Roth advises doctors working on kidney exchange programs and schools on their acceptance procedures. Moreover, some of the above-mentioned theoretical models are tested using actual data, for example by CBS (Centraal Bureau Voor de Statistiek) and CBP (Centraal Planbureau) in the Netherlands. 
Anna Zseleva was born on 2 December 1989 in Varna, a city on the Bulgarian Black Sea coast. She seemed to have always been interested in mathematics, and so she specialised in it during high school, and graduated at Berzsenyi Dániel Gimnázium in 2008, in Budapest, Hungary. Not being sure what grown-up mathematicians did, she chose studying economics. However, she carefully chose the economics programme with the most mathematicsrelated courses. She received her BSc and MSc diplomas in Quantitative Economic Analysis from Corvinus University of Budapest, where she graduated in 2013. She liked her Analysis and Measure Theory courses, which turned out to be useful later on. During her studies she realised that game theorists apply mathematics to many types of human interactions, which fit her interests perfectly. During her graduate studies she won both a university and a national research competition in game theory under the supervision of Miklós Pintér. This naturally led to a desire to pursue a PhD. Between 2013 and 2017 she was a PhD candidate at the Quantitative Economics Department of Maastricht University under the supervision of János Flesch and Dries Vermeulen. During this time many colleagues have mistaken her for a mathematician. In hindsight, it might have been easier if she had studied to be one, instead of having to correct them. The results of her $\mathrm{PhD}$ research are presented in this thesis. Anna presented these papers at various international conferences, and one of them is published at an international peer-reviewed journal, Games and Economic Behavior. 